\title{
Lower Granite GIS Data Description and Collection Guidelines
}
J. L. Gordon
B. J. Evans
E. M. Pèrry

December 1995

Prepared for the U.S. Department of Energy. under Contract DE-AC06-76RLO 1830

\section{Pacific Northwest Laboratory} Operated for the U.S. Department of Energy by Battelle Memorial Institute 


\section{DISCLAÎMER}

This report was prepared-as an account of work sponsored by an agency of the United States Governméent. Neither thé Unitéd States Government nor any agency thereof, nor Battelle Memorial Institute, nór any of their employees, makes any. warranty, express of implied, or assumes any legal liability or responsibility for the accuracy, completeness, or usefulness of any information, apparatus, product, or process disclosed, or represents that its use would not infringe privately owned rights. Reference hèrein to any specific commercial product, process', or service by trade name, trademark, manufacturer, or otherwise does not necessarily constitute or imply its endorsement, recommendation, or favoring by the United States Government or any agency thereof, or Battelle Memorial institute. The views and opinions of authors expressed herein do not necessarily state or reflect those of the United States Government or any agency thereof.

\section{PACIFIC NORTHWEST NATIONAL LÁBORĀTORY operated by \\ BATTELLE \\ iór the \\ UNITED STATES DEPARTMENT OF ENERGY under Contract DE-AC06-76RLO-1830.}

Printed in the United States of America

2. Available to DOE and DOE contractórs from the

Office of Scientific and Technical Information, P.O. Box 62, Oak Ridge, TN 37831; prices avaiiable from (615) $576-8401$.

Available to the public from the National Technical lnformation Service, U.S. Department of Commerce, 5285 Port Royal Rd., Springfield, VA 22161 


\title{
Lower Granite GIS Data Description and Collection Guidelines
}

\author{
J. L. Gordon \\ B. J. Evans \\ E. M. Perry
}

December 1995

\section{Prepared for}

the U.S. Department of Energy

under Contract DE-AC06-76RLO 1830

Pacific Northwest Laboratory

Richland, Washington 99352

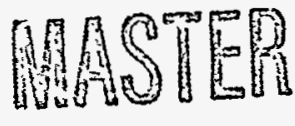





\section{Acronyms}

$\begin{array}{ll}\text { CERL } & \text { Construction Engineering Research Laboratory } \\ \text { GIS } & \text { Geographic Information System } \\ \text { GPS } & \text { Global Positioning System } \\ \text { GRASS } & \text { Geographic Resources Analysis Support System } \\ \text { NOAA } & \text { National Oceanic and Atmospheric Administration } \\ \text { PNL } & \text { Pacific Northwest Laboratory } \\ \text { QA } & \text { quality assurance } \\ \text { USCOE } & \text { U.S. Army Corps of Engineers } \\ \text { USGS } & \text { U.S. Geological Survey } \\ \text { UTM } & \text { Universal Transverse Mercator }\end{array}$





\section{Contents}

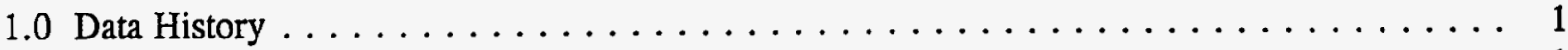

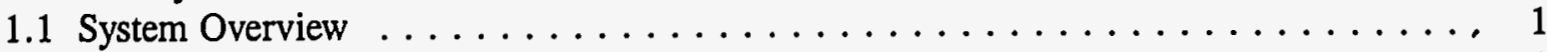

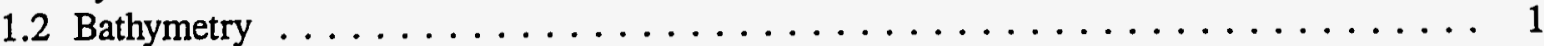

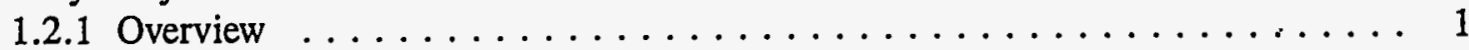

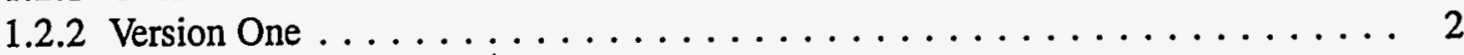

1.2 .3 Version Two . . . . . . . . . . . . . . . . . . . . . . . . 2

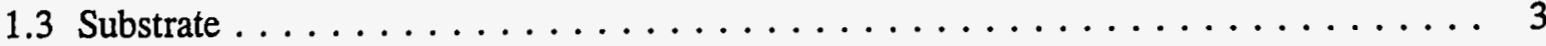

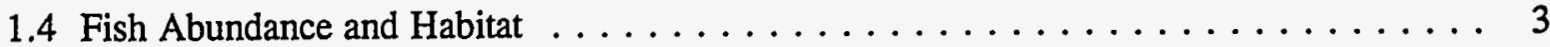

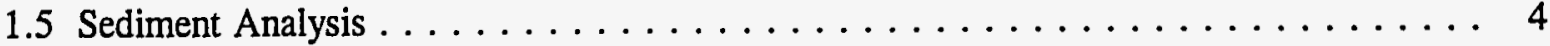

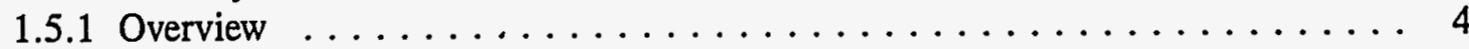

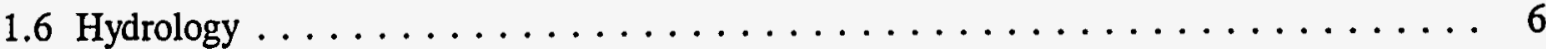

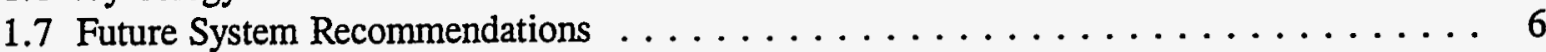

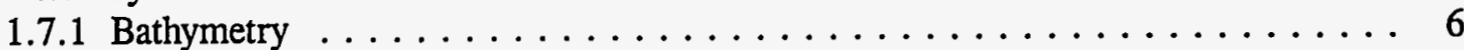

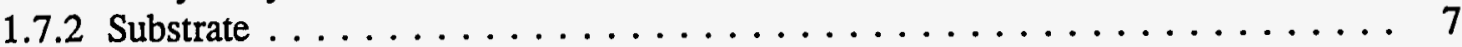

1.7.3 Fish Distribution and Abundance $\ldots \ldots \ldots \ldots \ldots \ldots \ldots \ldots \ldots$

1.7 .4 Sediment Analysis . . . . . . . . . . . . . . . . . . 9

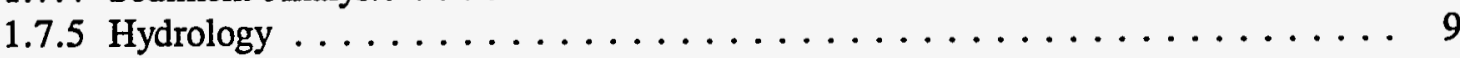

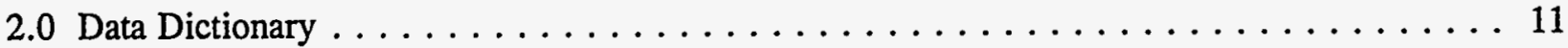

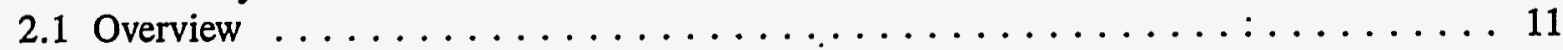

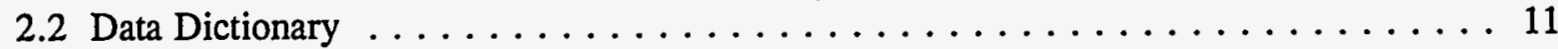

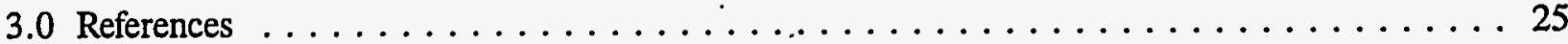

Appendix A - Conversion of Water Depth Contours to Elevations for PV-WAVE . . . . . . . 27

Appendix B - Conversion of Shoreline Monolith Data to Latitude, Longitude . . . . . . . . . . 31

Appendix C - Example from USCOE HEC-2 Modeling Runs . . . . . . . . . . . . . . 43

Appendix D - Subregions of the Lower Granite Reservoir . . . . . . . . . . . . . . . . . . . 149

Appendix E - Conversion of GRASS Raster Files to PV-WAVE Input $\ldots \ldots \ldots \ldots \ldots$

Appendix F - Interpolation of Depth Values for Channel Centerline . . . . . . . . . . . . 159

Appendix G - Conversion of Centerline Countours to GRASS Sites File . . . . . . . . . . . 173

Appendix H - Replacement of 1989 Sounding Data Using 1992 Hydroacoustic Data for

Schultz Bar and the Dredge Disposal Site, Creation of xyz Coordinates

from Soundings . . . . . . . . . . . . . . . . . . . . . 177

Appendix I - Replacement of 1989 Sounding Data Using 1992 Hydroacoustic Data for Schultz Bar and the Dredge Disposal Site, Creation of Higher Resolution File . . . 187

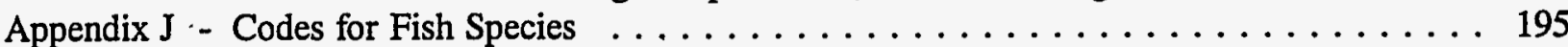

Appendix K - HEC-6 Sediment Properties . . . . . . . . . . . . . . . . . . . . . . 199 


\section{Figures}

1 Location of Bennett's Fish Sample Stations . . . . . . . . . . . . . . . 4

2 Example of Sediment Survey Locations Showing Single (Ellipse) and Composite (Polygon)

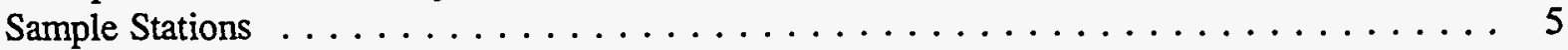

3 Photic Zones for the Centennial Island Reach, Based on the Lower Granite Bathymetry and

Elevation from $\mathrm{HEC}-2$ Simulations $\ldots \ldots \ldots \ldots \ldots \ldots \ldots \ldots \ldots \ldots$ 


\subsection{Data History}

\subsection{System Overview}

The Lower Granite Geographic Information System (GIS) was developed jointly by the U.S. Army Corps of Engineers (USCOE) Walla Walla District, and the Pacific Northwest Laboratory (PNL). ${ }^{\text {(a) }}$ The study area encompasses that portion of the Snake River extending from Illia (river mile 100.5) to the confluence of the Clearwater River (river mile 142). The goal of the project is to use GIS technology to analyze impacts of the drawdown mitigation option on the physical and biological environment of the Lower Granite Reservoir. The drawdown mitigation option is based on the hypothesis that faster juvenile salmon travel to the ocean would result in higher juvenile survival and greater smolt-to-adult return ratios; to accomplish this, reservoir elevations would be lowered to increase channel velocities. Altering the elevation of the reservoirs on the Snake River is expected to have a variety of impacts to the physical environment including changes to water velocity, temperature, dissolved gases, and turbidity. The GIS was developed to evaluate these changes and the resulting impacts on the anadromous and resident fish of the Snake River, as well as other aquatic organisms and terrestrial wildlife residing in the adjacent riparian areas.

The Lower Granite GIS was developed using commercial hardware and software (Intergraph Corp., Huntsville, Alabama), and is supported by a commercial relational database (ORACLE Corp., San Francisco, California). Much of the initial system development involved collecting and incorporating data describing the river channel characteristics, hydrologic properties, and aquatic ecology. Potentially meaningful data for the Lower Granite GIS were identified and an extensive data search was performed; however, approximately half of the data inventoried was not usable. Data in both digital and text formats were obtained from scientists across the Pacific Northwest who are analyzing the habitats, limnology, and hydrology of the Snake River. The next six sections of this document describe the bathymetry, fish abundance, substrate, sediment chemistry, and channel hydrology data. Recommendations for future sampling campaigns and a data dictionary follow the data description.

\subsection{Bathymetry}

\subsubsection{Overview}

The first task in the Lower Granite GIS was to create a digital model of the bathymetry of the Lower Granite Reservoir. An extensive search for bathymetry data revealed a lack of existing digital contour data, so PNL created the bathymetric model using the most recent data available. There were two versions of the bathymetry created. The first version used National Oceanic.and Atmospheric Administration (NOAA) navigational charts, and 1989 hydroacoustic soundings of river cross-sections taken in 0.5 to 1 mile intervals from Illia to the Clearwater confluence. When additional data became available, a second version was created by modifying the first version with additional data: 1) 1992 hydroacoustic sounding data, 2) detailed sounding data of Schultz Bar and the deep-dredge disposal site

(a) Pacific Northwest Laboratory is operated for the U.S. Department of Energy by Battelle Memorial Institute under contract DE-AC06-76RLO 1830. 
(river mile 120.5), and 3) $1 \mathrm{ft}$ bathymetry contour lines of the Snake-Clearwater River confluence that were digitized from aerial photographs taken during the 1992 drawdown study. References and detailed descriptions for these procedures follow.

The database tables related to bathymetry are bath_aspect_cent, bath_elev_cent, bath_slope_d_cent, bath_slope_p_cent, depth_cent, and photic_cent.

\subsubsection{Version One}

The first version was based on U.S. Geological Survey (USGS) Navigational Charts. All of the point depths available on the charts were digitized. The contour line representing $738 \mathrm{ft}$ above mean sea level, and contour lines representing underwater depths of $3,6,12,18$, and $30 \mathrm{ft}$ were also digitized. Water depth contours were converted to elevations above mean sea level to prepare the data for input to PV-WAVE (Visual Numerics, Boulder Colorado); the PV-WAVE script is shown in Appendix A. In addition to the NOAA charts, hydroacoustic soundings collected in 1989 were provided by the USCOE, Walla Walla District. The cross-sections were taken in 0.5 to 1 mile intervals from Illia to the Clearwater confluence, but did not contain georeferenced coordinates in latitude and longitude. The program read_hec_2xy.f was used to read these data, convert shoreline monolith information to latitude and longitude coordinates, and perform initial interpolation of the cross-section. This program can be found in Appendix B along with an example of the profile data in Appendix C. The Lower Granite Reservoir area had to be divided into eight sub-regions to allow PV-WAVE interpolation of the data. This was done using the program MAKE_WAVE.f (Appendix E), and the sub-region coordinates can be found in Appendix D. Once the interpolation was complete, the eight files were merged using the GRASS (Geographic Resources Analysis Support System; USCOE, Construction Engineering Research Laboratory [CERL]) software. Because interpolation programs do not preserve the anisotropy of river data, the interpolation program created discontinuous "deep holes" in the bathymetry at each digitized point. To avoid these erroneous interpolation results, three channel centerlines were digitized based on the NOAA charts. Channel centerline elevations were interpolated from a subset of the bathymetry points, contour lines, and cross-section data that were located in proximity of the channel centerline using the program tgridgen_xypts.f (Appendix F). The resulting contour file was used as input for the program XYZ TO GRPTS, which creates a GRASS sites file for display (see Appendix G). Another interpolation was performed with the improved channel bathymetry, after converting the gridpoints for use by PV-WAVE using MAKE_WAVE.f. The grid data from the final PV-WAVE interpolation were converted to vectors using GRASS software.

\subsubsection{Version Two}

For the second version, three additional data sets were merged into the existing model: 1) 1992 hydroacoustic Doppler soundings, 2) detailed soundings of Schultz Bar and the deep water dredge disposal site at river mile 120.5 , and 3 ) $1 \mathrm{ft}$ bathymetry contours created from aerial photography taken during the 1992 drawdown. All of these data were provided by the USCOE, Walla Walla District. Where there were data overlap, the 1992 hydroacoustic sounding data replaced the 1989 sounding data. The recent detailed soundings of Schultz Bar and the dredge disposal site replaced the older data; the data replacement programs can be found in Appendices $\mathrm{H}$ and $\mathrm{J}$. The $1 \mathrm{ft}$ bathymetry contour lines in the Snake-Clearwater confluence were digitized by the USCOE. Because of extreme noise in the $1 \mathrm{ft}$ contour lines, only the $5 \mathrm{ft}$ contour lines were used for the interpolation. To remove noise from the $5 \mathrm{ft}$ contour lines, the data were imported into Arc/Info (ESRI, Redlands, California), gridded and resampled to $20 \mathrm{ft}$ contours, and exported to DXF format. This DXF file was later merged with the 
second version of the bathymetry file. Once the bathymetric model had been generated, the surface modeling features of Intergraph were used to create aspect, elevation, degrees of slope, and percent of slope layers. The tables linked to these layers are bath_aspect_cent, bath_elev_cent, bath_slope_d_ cent, and bath_slope_p_cent.

\subsection{Substrate}

Substrate data were collected as part of the 1992 drawdown study (Curet 1993). Much of the data has not been published, but was provided by Curet. The substrate information collected included substrate type, degree of embeddedness, and the amount of cover from predation.

To incorporate this.data in the GIS, sampling areas were represented by polygons 0.5 miles long and $30 \mathrm{ft}$ wide; these polygon covered elevations 708 to $738 \mathrm{ft}$, and ranged from river mile 107 to 139, along both shorelines. The polygons centroids were then linked to the substrate database tables by river mile and shore direction. Within each polygon, the data reported are substrate type, embedded quartile, and cover quartile. Substrate type is divided into .9 classes: 1) fines $(<2 \mathrm{~mm}), 2)$ small gravel (2 to $25 \mathrm{~mm}), 3$ ) medium gravel $(25$ to $50 \mathrm{~mm})$, 4) large gravel $(50$ to $75 \mathrm{~mm}$ ), 5) small cobble ( 75 to $150 \mathrm{~mm}), 6)$ medium cobble (150 to $225 \mathrm{~mm}), 7$ ) large cobble (225 to $300 \mathrm{~mm}), 8)$ small boulder (300 to $600 \mathrm{~mm}$ ), and 9) large boulder (>600 mm). The substrate degree of embeddedness is expressed as quartiles ( 0 to $25 \%, 25$ to $50 \%, 50$ to $75 \%$, and 75 to $100 \%$ ). Cover quartile describes the percent of area with cover from predation. The data resides in the database tables curet_substrat_ stn and curet substrate. In addition to the tabular substrate information for each sampling point, a video frame that characterized the habitat was captured and stored for each of the polygons. Instructions on how to retrieve videos can be found in the Intergraph I/RAS C User's Guide.

\subsection{Fish Abundance and Habitat}

As part of a sediment dredging and in-water disposal study, physical and biological data were collected to determine the impacts on fish habitat in the Lower Granite Reservoir; these data were published as a series of USCOE reports (Bennett and Shrier 1986; Bennett et al. 1988; Bennett et al. 1990; Bennett et al. 1991; Bennett et al. 1993). Physical data on the water velocity, dissolved oxygen, temperature, turbidity, and substrate were collected at a series of fish sampling stations extending from river mile 110 to river mile 128 . In addition to the water physical properties, fish catch data for approximately 30 fish species were recorded for these sampling stations. The fish sampling techniques used included beach seine, electrofish, arid gill net. The age of the fish (yearling or subyearling) was indicated as well as stock-type (wild or hatchery): Fish passage counts from the Lower Granite Dam from 1987 to 1992 were also included in the database.

All of the fish abundance and physical data were obtained from Bennett (University of Idaho) in computer spreadsheet form, and loaded into the relational database. Fish sampling stations were determined from maps contained within the published reports, and were represented in the GIS as a point sample (Figure 1). The fish catch data were normalized using 'catch per unit effort;' see the description in the Data Dictionary, 'bennett_stn_counts.' 


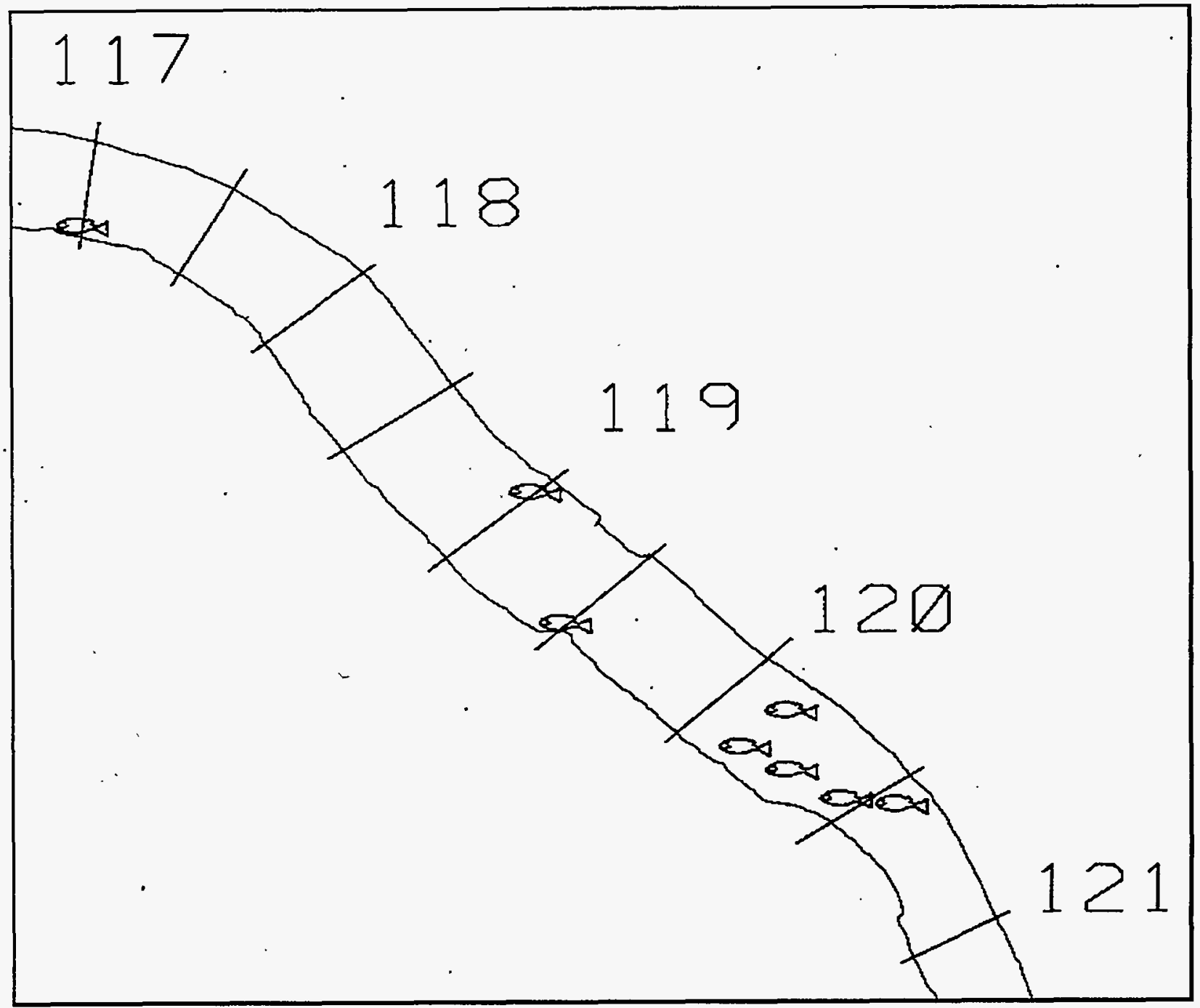

Figure 1. Location of Bennett's Fish Sample Stations (1988 to 1991)

The fish sample stations in the GIS are linked to the database by station numbers. Sample dates may be queried by month, year, or julian day (day of year 1 to 365). Species may be queried by the species code; these codes are included in Appendix I. The database tables that store the fish distribution and habitat data are bennett_stn_prop, bennett_survey_stn, bennett_stn_counts, fish_passage, and fish_pass_counts.

\subsection{Sediment Analysis}

\subsubsection{Overview}

In support of the 1992 drawdown study, the USCOE requested PNL to analyze potential water quality impacts on dredge areas in the Snake and Columbia Rivers, and the effects of resuspension of 
sediments in the Snake River as a result of drawdown. Sampling and analysis of sediments along key Ports of Authority on the Columbia and Snake rivers was performed by the Battelle Marine Sciences Laboratory in Sequim, Washington (Pinza et al. 1992). Data included in the Lower Granite database range from below the dam (river mile 104) to the confluence of the Clearwater River (river mile 139). Most of the sampling occurred in August 1991; analysis of river mile 119.56 was performed on a composite of archived core samples collected in October 1990.

Sediment chemistry data were extracted from the published PNL report and loaded into the relational database; the sample sites were linked to graphical representations of the sample area boundaries by sample station numbers. The locations of sample stations were provided by Tom Miller, USCOE, Walla Walla District. All river mile numbers were reported in statute miles, for consistency with the Lower Granite database. Some samples were composited before analysis; these samples were represented as a polygon in the map layer (Figure 2). The uncomposited sample stations were represented by circles approximately 0.25 mile in diameter.

The chemical analyses reported in the database include:

- Conventionals (percentage of total volatile solids, total organic carbon, ammonia, phosphate, and sulfides)

- Oil and grease, and total petroleum hydrocarbons

- Metals (As, $\mathrm{Cd}, \mathrm{Cr}, \mathrm{Cu}, \mathrm{Pb}, \mathrm{Hg}, \mathrm{Zn}$ )

- Polynuclear aromatic hydrocarbons

- Chlorinated pesticides and polychlorinated biphenyls.

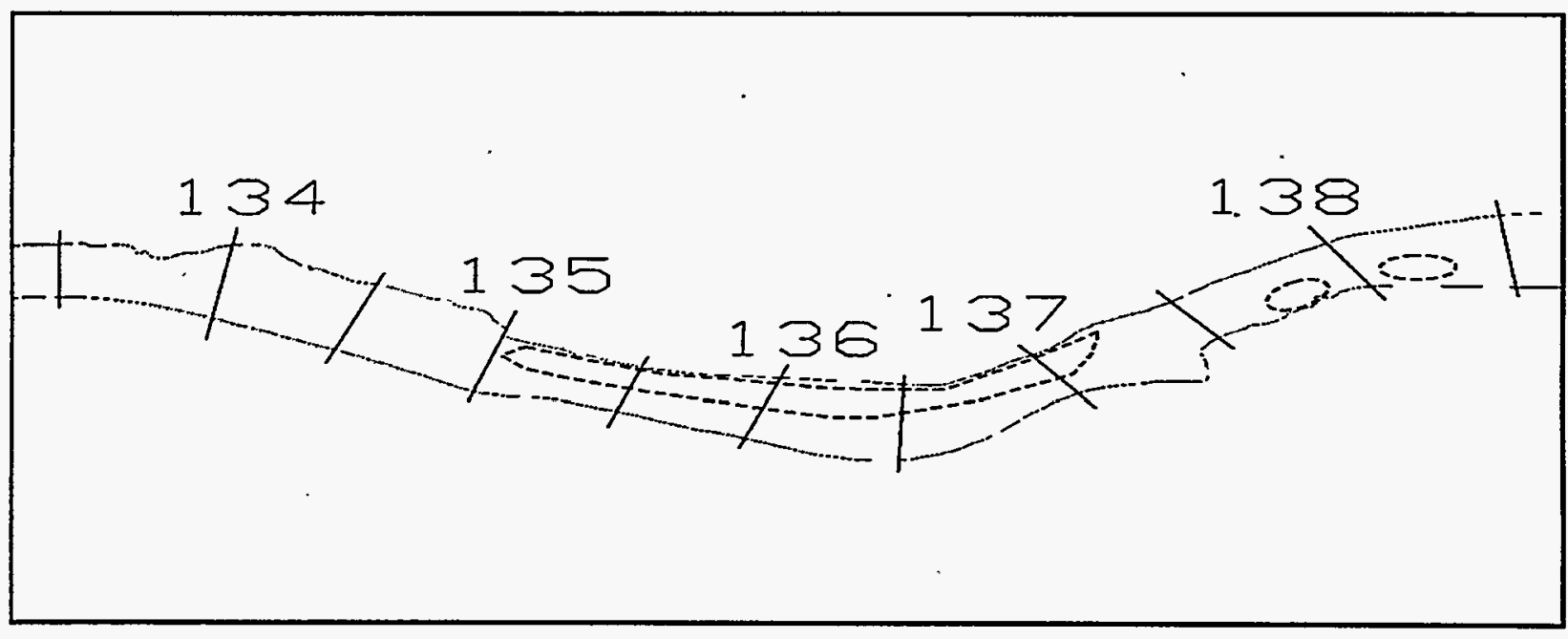

Figure 2. Example of Sediment Survey Locations Showing Single (Ellipse) and Composite (Polygon) Sample Stations 
Note that the quality assurance (QA) records are NOT included in the database, but each of the concentrations reported include a QA code. These codes refer to the table conc_qual_code that describes the concentration qualifier. The reference for the sediment chemistry data is recorded in the Data Reference table. Related database tables are sed_analy_tab_1, sed_chemistry, sed_survey_stn, conc_qual_code, and chem_qa.

\subsection{Hydrology}

To analyze the effects of the various drawdown options, a number of different types of hydrologic data were created for the Lower Granite GIS. For each of three discharge rates, 30,000, 60,000, and $140,000 \mathrm{cfs}$, six different operable pool options, 738, 733, 700,690, 681, and $623 \mathrm{ft}$ were examined. Each of these flow rate/operable pools constituted a drawdown scenario. For each drawdown scenario, a number of different data were generated. The surface of the river was determined by initializing HEC-2 (USCOE 1990) for that scenario, and given the river elevations calculated by HEC-2, interpolating throughout the channel based on $1 \mathrm{ft}$ contours of the bathymetry model. These polygons that represent the HEC-2 surface water profiles begin at river mile 107.5 and extend to river mile 139.5; individual polygons range from 0.25 to 2 miles in length. Travel times, velocity, pool elevations, and surface area are stored for each polygon. Related database tables are hec2 point, hec2_vel, and op_pool_cent.

While the surface water profiles derived from modelling were not as smooth as the natural river, it was a fast way to approximate the river surface within the GIS' vertical accuracy range. These river surfaces were then subtracted from the bathymetry to produce an isopach surface showing the depth of the reservoir for each drawdown scenario. These models were used to generate habitat (photic zone) change maps, bottom area calculations, and reservoir volume calculations. An example photic zone map for the Centennial Island reach is shown in Figure 3.

\subsection{Future System Recommendations}

\subsubsection{Bathymetry}

Through merging of the most recent data, we believe we have created the most accurate bathymetric data for the Lower Granite Reservoir. The creation of the bathymetry model was slow and tedious because of different data formats and the development of programs to manipulate the data. To increase the accuracy and reduce the cost of updating, we recommend that the following modifications be made to the sampling procedures:

1. Record each transect starting and ending point in latitude and longitude using Global Positioning System (GPS) technology. Latitude and longitude were not available for each of the cross-section points collected for the surface water modelling (HEC-2). Calculations were performed using known positions of shoreline monoliths; however, it was tedious and possibly not as accurate.

2. Decrease the amount of data collected with hydroacoustic soundings. Sounding files existed that would have been beneficial to incorporate into the bathymetric data; however, they were 


\section{CENTENNIAL ISLAND REACH}

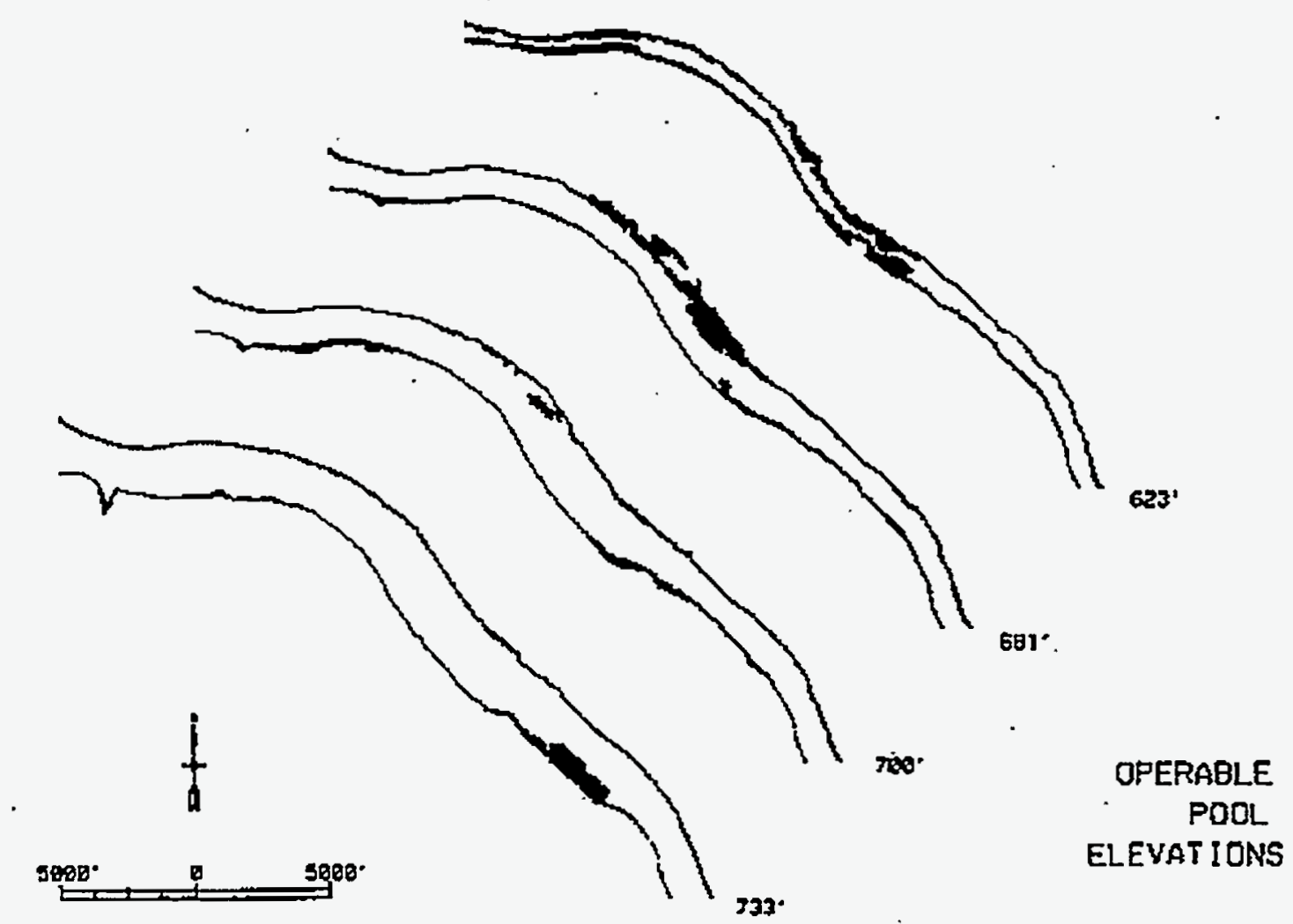

Figure 3. Photic Zones for the Centennial Island Reach, Based on the Lower Granite Bathymetry and Elevation from HEC-2 Simulations

excluded because the volume of data exceeded software limits and time constraints. This could have been avoided by decreasing the frequency of data capture during the sounding: these files captured approximately 60 soundings per second, and the capture rate could have been reduced by half without significantly affecting accuracy.

3. Explore technological advances for obtaining bathymetry. This includes aerial flights equipped with sonar and software packages like GeoLink (GeoLink Corp., Missoula, Montana) and BioSonics (BioSonics, Seattle, Washington) that will interface with GIS and hydroacoustic equipment.

\subsubsection{Substrate}

Substrate data were collected by Tom Curet (USCOE) and Dr. David Bennett (University of Idaho). The data were scattered point samples, and large portions of the river were unsampled. 
Because interpolation of the substrate data was not feasible due to the data distribution, these data were of limited use in characterizing habitat. Other available substrate data were not used because of incompatible classification techniques. Recommendations for improving substrate data are:

1. Take substrate measurements in 5 to $10 \mathrm{ft}$ intervals in crucial areas. It may be possible to interpolate substrate information at these intervals using additional information such as water velocity. In certain areas, use aerial photography to digitize underwater features (e.g., sandbars) and derive substrate information.

2. Standardize methods of collecting substrate data. It is recommended that future substrate data be gathered using the HEC- 6 Classifications of Sediment Properties and Transport Functions. These classifications can be found in Appendix $\mathrm{K}$.

3. For video and other photographic data collection, include a field notebook or other object for scale. This is especially important when characterizing surface/subsurface properties (e.g., substrate, vegetation, etc.).

4. Record each frame (or time for video) and the location on a log sheet.

5. Use differential GPS (within $2.5 \mathrm{~m}$ accuracy) for locating data points whenever possible.

\subsubsection{Fish Distribution and Abundance}

Monthly count/effort data were calculated using Dr. Bennett's data stored in a Lotus 1-2-3 (Lotus Development Corporation) spreadsheet. These data required manipulation before being loaded into ORACLE. Several suggestions that would reduce the amount of time required for data preparation are listed below:

1. Standardize fish station numbers. When entering data into the spreadsheet, pay close attention to syntax and case.

2. Create a LOTUS macro that calculates monthly catch/effort. When Dr. Bennett provides updates, there would be no need to have the individual records for each fish sampling effort; only one record per month would be required; this would reduce the processing time, space requirements, and the possibility of errors.

The ability to interpolate fish counts beyond the sampling station was desired; however, the sparsity of the sampling stations precluded interpolation of the data or calculations of volumetric fish counts. Several suggestions that may make these abilities feasible:

1. Explore sampling techniques that sample fish volumetrically, such as water column sampling.

2. Position fish sampling stations closer together. If stations are approximately $100 \mathrm{ft}$ apart, interpolation is feasible. 


\subsubsection{Sediment Analysis}

Because of the methods that were used to collect the sediment samples and report the analyses, inclusion of the data in the database was made more difficult. In particular, digitizing the sample areas in the GIS was difficult because many of the sampling stations were composited. The following guidelines would improve the data quality and expedite incorporation into the database and GIS:

1. Don't composite samples unless absolutely necessary; analysis of individual samples may provide more information, and samples may still be composited after individual analysis.

2. Sample locations should be well-defined in advance, and increased accuracy of sample locations can be achieved using differential GPS.

3. Analyze the samples for particle size if possible; this would supplement substrate information in the database.

\subsubsection{Hydrology}

Hydrologic data such as velocity were desired. Transects of the river were, at times, many miles apart, which precluded interpolation of the data. Recommendations to improve hydrologic data include:

1. Run transects in spacing equal to the width of the river channel. Georeference the starting and ending position of the boat. A test case was done using transects $25 \mathrm{ft}$ apart; this data was easily interpolated, but data at this resolution does not exist for the entire reservoir.

2 Include velocity measurements in all field sampling efforts if the collection of the data does not increase field time or cost. 



\subsection{Data Dictionary}

\subsection{Overview}

ORACLE software comprises the relational database used for the Lower Granite GIS. All tables have been normalized for more efficient storage, retrieval, and updating.

The following section describes the database tables and fields. There are two types of tables that can exist in Intergraph: 1) tables with graphic elements linked to them, and 2) tables that do not have graphic elements linked to them. For the first case, Intergraph requires the fields mapid and mslink to be present. Mslink is always defined as an integer without nulls and mapid is defined as an integer with nulls allowed. More details on these fields can be found under the specific table entries following this overview. The second type of table is used for relational joins to retrieve information from other tables.

The format for dictionary entries is tablename, fieldname, data type, and either a "not null" notation or blank. "Not null" means the field is a key field and blanks are not allowed. If "not null" does not appear after the fieldname, blanks are acceptable for that field.

NOTE: All river mile distances are defined in statute miles, unless otherwise indicated. "Julian Day" is actually day of the year: 1 to 365 for non-leap years and 1 to 366 for leap years.

\subsection{Data Dictionary}

\section{TABLE: bath_aspect_cent}

Defines the minimum and maximum aspect of the bathymetry. Linked to a graphic centroid.

FIELD: mslink (integer) not null

An Intergraph required field for tables that contain graphic elements. A unique value that links graphic elements to the database.

FIELD: mapid (integer)

An Intergraph required field for tables that contain graphic elements. Identifies the map (design file) that contains the graphic element associated with the database record.

FIELD: aspect_min (double)

Minimum aspect (degrees) in polygon.

FIELD: aspect_max (double)

Maximum aspect (degrees) in polygon. 
Defines the minimum and maximum elevation of the bathymetry. Linked to a graphic centroid.

FIELD: mslink (integer) not null

An Intergraph required field for tables that contain graphic elements. A unique value that links graphic elements to the database.

FIELD: mapid (integer)

An Intergraph required field for tables that contain graphic elements. Identifies the map (design file) that contains the graphic element associated with the database record.

FIELD: elev_min (double)

Minimum elevation in polygon.

FIELD: elev max (double)

Maximum elevation in polygon.

TABLENAME: bath_slope_d_cent

Defines the minimum and maximum slope in degrees in the bathymetry. Linked to a graphic centroid.

FIELD: mslink (integer) not null

An Intergraph required field for tables that contain graphic elements. A unique value that links graphic elements to the database.

FIELD: mapid (integer)

An Intergraph required field for tables that contain graphic elements. Identifies the map (design file) that contains the graphic element associated with the database record.

FIELD: slope_min (double)

Minimum slope (degrees) in polygon.

FIELD: slope max (double)

Maximum slope (degrees) in polygon.

TABLENAME: bath_slope_p_cent

Defines the minimum and maximum slope in percent in the bathymetry. Linked to a graphic centroid. 
FIELD: mslink (integer) not null

An Intergraph required field for tables that contain graphic elements. A unique value that links graphic elements to the database.

FIELD: mapid (integer)

An Intergraph required field for tables that contain graphic elements. Identifies the map (design file) that contains the graphic element associated with the database record.

FIELD: slope_min (double)

Minimum slope (percent) in polygon.

FIELD: slope $\max$ (double)

Maximum slope (percent) in polygon.

TABLENAME: bennett_stn_counts

Contains fish count information obtained from Dr. Bennett, University of Idaho.

FIELD: fish_station_no ( small integer) - not null

Sampling fish station number as reported from Dr. Bennett's surveys. Key field used to join bennett_stn_prop and bennett_survey_stn tables.

FIELD: survey_month (small integer) - not null

Month that sample was taken. Key field used to join bennett stn prop and bennett_survey_stn tables.

FIELD: survey_year (small integer) - not null

Year that sample was taken. Key field used to join bennett_stn_prop and bennett_survey_stn tables.

FIELD: julian_day (small integer)

The number of the day of the year.

FIELD: sampling_method (char 15)

Method used to count fish. May be beach seine, gillnet, electrofish, or all gear.

FIELD: species (char 35)

Three letter abbreviation for species sampled. Abbreviations may be found in Appendix J.

FIELD: mo catch effort (double)

Catch per unit effort was determined (for Dr. Bennett's data) based on sample method, as follows.

- Bottom trawling, surface trawling and purse seining (towing): expressed as number of fish per meter of distance trawled/towed 
- Beach seining: expressed as number of fish per haul

- Electrofishing: number of fish per 5 minute pass

- Gill netting: number of fish per hour gill netting.

\section{TABLENAME: bennett_stn_prop}

Defines the hydrological and physical properties at the fish sampling station on the sample day. Data source is Dr. Bennett, University of Idaho.

FIELD: fish_station_no (small integer) - not null

Sampling fish station number as reported from Dr. Bennett's surveys. Key field used to join bennett_stn_counts and bennett_survey_stn tables.

FIELD: survey_month (small integer) - not null

Month that sample was taken. Key field used to join bennett_stn_counts and bennett_survey_stn tables.

FIELD: survey_year (small integer) - not null

Year that sample was taken. Key field used to join bennett_stn_counts and bennett_survey_stn tables.

FIELD: $\quad$ substrate (char 25)

Characterization of the dominant substrate type at the fish station on the sample day.

FIELD: velocity (double)

The speed at which the water is moving at the fish station on the sample day. If multiple samples were taken that month, velocity measurements were averaged.

FIELD: dissolved oxygen (double)

The dissolved oxygen measurement at the fish station on the sample day. If multiple samples were taken that month, dissolved oxygen levels were averaged..

FIELD: temperature (double)

The temperature in degrees fahrenheit at the fish station on the sample day. If multiple samples were taken that month, temperatures were averaged.

FIELD: turbidity (double)

Measurement of turbidity at the fish station on the sample day. If multiple samples were taken that month, turbidity measurements were averaged.

FIELD: julian_day (small integer)

The number of days that have elapsed since January 1st (i.e., February 1 st $=32$ nd day). 
Defines the physical location and properties of the Dr. Bennett's fish sampling station. Contains graphic link that identifies survey station location.

FIELD: mslink (integer) not null

An Intergraph required field for tables that contain graphic elements. A unique value that links graphic elements to the database.

FIELD: mapid (integer)

An Intergraph required field for tables that contain graphic elements. Identifies the map (design file) that contains the graphic element associated with the database record.

FIELD: fish_station_no (small integer) - not null

Sampling fish station number as reported from Dr. Bennett's surveys. Key field that links table to bennett_stn_prop and bennett_stn_counts.

FIELD: river_mile (double)

River mile of fish sampling station indicated in Dr. Bennett's surveys. If river mile was indicated as range, the mid-point was taken.

FIELD: year_constructed (small integer)

The year the fish sampling station was constructed, as indicated in Dr. Bennett's surveys.

FIELD: northing (double)

Universal Transverse Mercator (UTM) northing coordinate of fish sampling station obtained during field studies using a GPS'unit.

FIELD: easting (double)

UTM easting coordinate of fish sampling station obtained during field studies using a GPS unit.

FIELD: stn description (char 150)

Textual description of fish station location as found in Dr. Bennett's survey reports.

FIELD: year_first_sampled (small integer)

The year the fish station was first sampled.

FIELD: latitude (double)

The latitudinal position of the fish station.

FIELD: longitude (double)

The longitudinal position of the fish station. 
FIELD: naut_river_mile (double)

1 nautical mile $=1.151$ statute miles (Espenshade, Jr. and Morrison 1978).

TABLENAME: birds

Defines species and nest dates of birds and contains graphic link for identifying nest location.

FIELD: mslink (integer) not null

An Intergraph required field for tables that contain graphic elements. A unique value that links graphic elements to the database.

FIELD: mapid (integer)

An Intergraph required field for tables that contain graphic elements. Identifies the map (design file) that contains the graphic element associated with the database record.

FIELD: $\quad$ species (char 30) not null

FIELD: nest_date (char 30)

TABLENAME: conc_qual_code

FIELD: qualifier (char 2) not null

The concentration qualifier code which links the description below to the sediment analysis table (see sed_analy_tab_1).

FIELD: qual_description (char 200)

A description of the significance (data quality) of a reported chemical concentration.

TABLENAME: curet_substrat_stn

FIELD: mslink (integer) not null

An Intergraph required field for tables that contain graphic elements. A unique value that links graphic elements to the database.

FIELD: mapid (integer)

An Intergraph required field for tables that contain graphic elements. Identifies the map (design file) that contains the graphic element associated with the database record.

FIELD: river mile (real) not null

The sediment sampling location in statute river miles. 
FIELD: shore_direction (char 2) not null

This indicates whether the sampling location was on the north $(N)$ or south $(S)$ shore of the river.

FIELD: naut_river_mile (real)

The sediment sampling location in nautical river miles, determined as 1.151 times the statute river mile.

TABLENAME: curet_substrate

FIELD: river_mile (real) not null

The statute river mile of the sampling location; the river mile and shore direction (below) link the records in this table to the curet_substrat_stn table.

FIELD: shore direction (char 2) not null

This indicates whether the sampling location was on the north $(N)$ or south $(S)$ shore of the river.

FIELD: size_range (char 20)

The particle size range (in $\mathrm{mm}$ ) of the exposed gravel and sediments.

FIELD: substrate_type (char 30)

The particle type (fine grains, cobbles, etc.).

FIELD: embedded quartile (small integer)

The degree of embeddedness (large particles embedded in finegrains) expressed in terms of $0-25 \%$ (recorded as 1 ), $25-50 \%$ (2), etc.

FIELD: cover_quartile (small integer)

The amount of cover from predation (boulders, tree limbs, etc.) expressed in terms of $0-25 \%$ (recorded as 1 ), $25-50 \%$ (recorded as 2 ), etc.

FIELD: area_of polygon (real)

The area contained by the sampling area.

TABLENAME: depth_cent

FIELD: mslink (integer) not null

An Intergraph required field for tables that contain graphic elements. A unique value that links graphic elements to the database. 
FIELD: mapid (integer)

An Intergraph required field for tables that contain graphic elements. Identifies the map (design file) that contains the graphic element associated with the database record.

FIELD: oper_pool_elev (small integer)

Operating pool elevation (in feet) of Lower Granite Dam.

FIELD: flow rate kcfs (integer)

Rate of flow of Lower Granite Dam in kcfs.

FIELD: reservoir (small integer)

????

FIELD: depth_min (double)

Minimum depth (feet) in polygon under flow rate and operating pool elevation indicated.

FIELD: depth max (double)

Maximum depth (feet) in polygon under flow rate and operating pool elevation indicated.

TABLENAME: fish_pass_counts

Defines fish passage counts for Lower Granite Reservoir. Data source: USCOE.

FIELD: passage name (char 35) not null

Name given to area that fish pass through to reach Lower Granite Reservoir and the Snake

River. Key field used to link to table fish passage.

FIELD: julian_day (small integer) not null

The number of days that have elapsed since January 1st (i.e., February 1 st $=32$ nd day).

FIELD: survey_year (small integer) not null

Year that sample was taken.

FIELD: species (char 35) not null

3 letter abbreviation for species sampled. Abbreviations may be found in Appendix J.

FIELD: stock type (char 10)

Describes stock type of fish being either wild or hatchery.

FIELD: age (char 15)

Describes age of fish being either yearling or subyearling.

FIELD: fish_count (double)

Number of fish counted at passage point within a specific year, day, and species. 
FIELD: flow (double)

Flow rate in kcfs, of Lower Granite Dam on the day the specific count was taken.

FIELD: temperature (double)

Temperature at the passage on the day of the fish count.

FIELD: turbidity (double)

Turbidity at the passage on the day of the fish count.

TABLENAME: fish_passage

Table that contains the fish passage name and description and graphic link to identify fish station location. Data source: USCOE.

FIELD: mslink (integer) not null

An Intergraph required field for tables that contain graphic elements. A unique value that links graphic elements to the database.

FIELD: mapid (integer)

An Intergraph required field for tables that contain graphic elements. Identifies the map (design file) that contains the graphic element associated with the database record.

FIELD: passage name (char 35) not null

Name given to area that fish pass through to reach Lower Granite Reservoir and the Snake River. Key field used to link table to fish_pass_counts.

FIELD: passage_desc (char 35)

Description of area that fish pass through to reach Lower Granite Reservoir and the Snake River.

TABLENAME: half_mile_info

A tables used to define half-mile markers along river.

FIELD: mslink (integer) not null

An Intergraph required field for tables that contain graphic elements. A unique value that links graphic elements to the database.

FIELD: mapid (integer)

An Intergraph required field for tables that contain graphic elements. Identifies the map (design file) that contains the graphic element associated with the database record. 
FIELD: naut_river_mile (double) not null

Nautical river mile number. 1 nautical mile $=1.151$ statute miles (Espenshade, Jr. and Morrison 1978). Used for labels in graphics.

FIELD: stat_river_mile (double)

Statute river mile number. Used for labels in graphics.

TABLENAME: hec 2 point

FIELD: mslink (integer) not null

An Intergraph required field for tables that contain graphic elements. A unique value that links graphic elements to the database.

FIELD: mapid (integer)

An Intergraph required field for tables that contain graphic elements. Identifies the map (design file) that contains the graphic element associated with the database record.

FIELD: statute_mile (double) not null

Statute river mile number of graphic point. Used for reference in hydrological map layers.

FIELD: naut_river_mile (double)

One nautical mile $=1.151$ statute miles (Espenshade, Jr. and Morrison 1978).

FIELD: oper_pool_elev (small integer)

Operating pool elevation (in feet) of Lower Granite Dam.

FIELD: flow_rate_kcfs (integer)

Rate of flow of Lower Granite Dam in kcfs.

TABLENAME: hec2_vel

Defines hydrological information from Hec-2 software.

FIELD: mslink (integer) not null

An Intergraph required field for tables that contain graphic elements. A unique value that links graphic elements to the database.

FIELD: mapid (integer)

An Intergraph required field for tables that contain graphic elements. Identifies the map (design file) that contains the graphic element associated with the database record.

FIELD: statute mile (double) not null

Statute river mile number of graphic point. Used for reference in hydrological map layers. 
FIELD: oper_pool_elev (small integer)

Operating pool elevation (in feet) of Lower Granite Dam.

FIELD: naut_river_mile (double)

Nautical river mile number. Used for reference in hydrological map layers. 1 nautical mile $=1.151$ statute mile (Espenshade, Jr. and Morrison 1978).

FIELD: flow_rate_kcfs (integer)

Rate of flow in kcfs of Lower Granite Dam used for modelling the hec2 run.

FIELD: river_mile

The cross section river mile, in statute miles.

FIELD: travel time (double)

Amount of time in hours that it takes to travel from river mile A to river mile B.

FIECD: velocity (double)

Average velocity in kcfs of the segment of river defined by the polygon.

FIELD: surface_area (double)

Surface area in acres of the segment of river defined by the polygon.

FIELD: pool_elev (double)

Average pool elevation of the segment of river defined by the polygon.

FIELD: pct chg velocity (double)

Percent of change in average velocity from river mile $A$ to river mile $B$.

FIELD: dist_btwn_hec_rm (double)

The distance in miles between river mile A and river mile B. The Hec2 programs divide the river into varying lengths.

FIELD: velo_difference (double)

Difference in velocity in $\mathrm{kcfs}$ of river mile $\mathrm{A}$ and river mile $\mathrm{B}$.

FIELD: diff div dist (double)

The velocity difference divided by the distance between HEC-2 river mile A and $\mathrm{B}$.

FIELD: area_of_polygon (double)

Area in feet of specific polygon.

TABLENAME: mammals

Defines the mammal species and location. 
FIELD: mslink (integer) not null

An Intergraph required field for tables that contain graphic elements. A unique value that links graphic elements to the database.

FIELD: mapid (integer)

An Intergraph required field for tables that contain graphic elements. Identifies the map (design file) that contains the graphic element associated with the database record.

FIELD: $\quad$ species (char 30) not null

The Genus and species name of the mammal.

TABLENAME: op_pool_cent

Defines operating pool and flow rate of Lower Granite Dam and contains graphical centroid.

FIELD: 'mslink (integer) not null

An Intergraph required field for tables that contain graphic elements. A unique value that links graphic elements to the database.

FIELD: mapid (integer)

An Intergraph required field for tables that contain graphic elements. Identifies the map (design file) that contains the graphic element associated with the database record.

FIELD: oper_pool_elev (small integer) not null

Operating pool elevation (in feet) of Lower Granite Dam.

FIELD: flow_rate_kcfs (integer) not null

Flow rate $(\mathrm{kcfs})$ of Lower Granite Dam.

TABLENAME: photic_cent

Defines the photic zones under a certain operating pool elevation. Linked to a graphic centroid.

FIELD: mslink (integer) not null

An Intergraph required field for tables that contain graphic elements. A unique value that links graphic elements to the database.

FIELD: mapid (integer)

An Intergraph required field for tables that contain graphic elements. Identifies the map (design file) that contains the graphic element associated with the database record.

FIELD: oper_pool_elev (small integer)

Operating pool elevation (in feet) of Lower Granite Dam. 
TABLENAME: sed_analy_tab_1

Contains detailed information on the sediment analysis results.

FIELD: 'sampling_stn (char 25) not null

The name of the sediment chemistry sampling station; this field links these records to the sed_survey_stn table.

FIELD: chemical (char 50) not null

The chemical being reported in this record; each chemical analysis for each sample is reported in a separate record.

FIELD: ' sample type (char 10)

This field indicates whether the sample was a normal sample, or a type of QA record (such as trip blanks, field replicates, etc.).

FIELD: concentration (real)

The chemical concentration reported for this sample.

FIELD: conc_units (char 25)

The units of that the chemical concentration are reported in.

FIELD: conc qa (char 10)

The $\overline{Q A}$ code which describes the accuracy of the concentration; this code is linked to the conc_qual_code table.

FIELD: comments (char 100)

This field contains special notes, comments, etc.

TABLENAME: sed_survey_stn

Defines the location of the sediment chemistry sampling station.

FIELD: mslink (integer) not null

An Intergraph required field for tables that contain graphic elements. A unique value that links graphic elements to the database.

FIELD: mapid (integer)

An Intergraph required field for tables that contain graphic elements. Identifies the map (design file) that contains the graphic element associated with the database record. 
FIELD: sampling_stn (char 25) not null

The name of the sediment chemistry sampling station; this field links these records to the sed_analy_tab_1 table.

FIELD: river_mile (real) not null

The statute river mile of the sediment chemistry sampling location.

FIELD: northing (real)

The Washington State Plane South zone Northing (in feet) of the sample station location.

FIELD: easting (real)

The Washington State Plane South zone Easting (in feet) of the sample station location.

FIELD: stn_description (char 75)

The description of the station location.

FIELD: latitude (real)

The latitude (in degrees) of the sample station location.

FIELD: longitude (real)

The longitude (in degrees) of the sample station location. 


\subsection{References}

Bennett, D. H., and F. C. Shrier. 1986. Effects of Sediment Dredging and In-Water Disposal on Fishes in Lower Granite Reservoir, ID - WA. Completion Report, prepared for the U.S. Army Corps of Engineers - Walla Walla District, Walla Walla, Washington.

Bennett, D. H., L. K. Dunsmoor, and J. A. Chandler. 1988. Fish and Community Abundance at Proposed In-Water Disposal Sites, Lower Granite Reservoir (1987). Completion Report, with Addendum, prepared for the U.S. Army Corps of Engineers - Walla Walla District, Walla Walla, Washington.

Bennett, D. H., J. A. Chandler, and L. K. Dunsmoor. 1990. Lower Granite Reservoir In-Water Disposal Test: Results of the Fishery, Benthic, and Habitat Monitoring Program - Year 1 (1988). Completion Report, prepared for the U.S. Army Corps of Engineers - Walla Walla District, Walla Walla, Washington.

Bennett, D. H., J. A. Chandler, and G. Chandler. 1991. Lower Granite Reserwoir In-Water Disposal Test: Results of the Fishery, Benthic, and Habitat Monitoring Program - Year 2 (1989). Completion Report, prepared for the U.S. Army Corps of Engineers - Walla Walla District, Walla Walla, Washington.

Bennett, D. H., T. J. Dresser, T. S. Curet, K. B. Lepla, and M. A. Madsen. 1993. Lower Granite Reservoir In-Water Disposal Test: Results of the Fishery, Benthic, and Habitat Monitoring ProgramYear 4 (1991). Completion Report, prepared for the U.S. Army Corps of Engineers - Walla Walla District, Walla Walla, Washington.

Bennett, D. H., M. A. Madsen, T. S. Curett, and T. J. Dresser. 1994. Monitoring the Fish Community Activity at Disposal and Reference Sites in Lower Granite Reserwir, Idaho - Washington Year 5 [1992]. Report prepared for the U.S. Army Corps of Engineers - Walla Walla District, Walla Walla, Washington.

Curet, T. S. 1993. Habitat Use, Food Habits, and the Influence of Predation on Subyearling Chinook Salmon in Lower Granite and Little Goose Reserwirs, Washington. Masters Thesis for the University of Idaho, Moscow, Idaho. $71 \mathrm{pp}$.

Espenshade, E. B., Jr., and J. L. Morrison (eds). 1978. Goode's Wbrld Atlas. Rand McNally. 372 pp.

Pinza, M. R., J. Q. Word, E. S. Barrows, H. L. Mayhew, and D. R. Clark. 1992. Snake and Columbia Rivers Sediment Sampling Project. PNL-8479, prepared for the U.S. Army Corps of Engineers - Walla Walla District by Pacific Northwest Laboratory, Richland, Washington.

U.S. Army Corps of Engineers. 1990. Water Surface Profiles Users' Manual. USCOE Hydrologic Engineering Center, Davis, California. 



\section{Appendix A}

Conversion of Water Depth Contours to Elevations for PV-WAVE 



\section{Appendix A}

\section{Conversion of Water Depth Contours to Elevations for PV-WAVE}

LG_grid=intarr $(3520,3170)$

openr, 1,'/files8/guy/tmp/bathymetry.739shore.V2'

readu, 1,LG_grid

close, 1

$\min z z=\min \left(L G \_g r i d\right)$

$\max _{-} z=\max \left(L_{G}\right.$ _grid)

interval $=[608,618,628,638,648,658,668,678,688,698,708,718,728,738]]$

contour,LG_grid,/follow,/spline,levels = interval,\$

path='whole_river_contour.PVout'

pnl_contour_out_12,'whole_river_contour.PVout', 'whole_river_contour_data' 



\section{Appendix B}

Conversion of Shoreline Monolith Data to Latitude, Longitude 



\section{Appendix B}

\section{Conversion of Shoreline Monolith Data to Latitude, Longitude}

process_hec_2xy.f

Read HEC-2 data and convert coordinates to latitude and longitude. Interpolate HEC-2 data crosssections.

implicit none

integer maxmon, maxsect, maxinsect, maxsectmon

parameter $\quad(\operatorname{maxmon}=150$, maxsect $=150$, maxinsect $=700$,

$\mathrm{C}$

$+\quad \therefore \quad$ maxsectmon $=8$ )

character*40 fnmono, fn89, fnout

character*80 buffer(maxsect), tembuf, xshead

character*160 sectbuff

integer iunmn, iun89, oun, linecount, rivmile, rivindex,

$+\quad$ nskip, $i, j$, ip

real rivermile(maxmon), xsrivermile, numstations,

$+\quad$ station_elev(maxinsect), station(maxinsect),

$+\quad$ start_station,end_station

integer monindex(maxmon),

$+\quad$ numrecs, num_extra_stations,

$+\quad$ station index, ìb,ie, xscount,

$+\quad$ curr_index, ic, rm, ind1, ind2, old_rm

real monelev(maxsectmon), diff, maxdiff,

$+\quad$ rivmaltb, noraltb, eastaltb, elevaltb, stationaltb

character*3 rivshorealtb

double precision $\operatorname{monx}(\operatorname{maxsectmon})$, mony(maxsectmon),

$+$

$+$

C

xend,yend,zend, start_dist, xout, yout

monstation(maxsectmon), xstart,ystart,zstart,

C endfile, found, same_rm, altformb

C read the file names and open the 1) monolith coordinate file, 2) the 1989 cross-sections file, and

C 3) the output file

C

iunmn $=11$

iun89 $=13$

oun $=21$

write $(*, *)$ ',

write $(*, *)$ ' Enter File Names' 
write $(*, *)$ ' Enter Monolith Coordinate File Name'

$\operatorname{read}(*, 100)$ fnmono

C

open(iunmn, file $=$ fnmono,status $=$ 'old')

write $(*, *)$ ' Enter Cross-Section File Name'

$\operatorname{read}(*, 100)$ fn 89

C

open(iun89, file $=$ fn89, status $=$ 'old')

write $(*, *)$ ' Enter output File Name'

$\operatorname{read}(*, 100)$ fnout

C

open(oun, file $=$ fnout, status = 'unknown')

endfile $=$.false .

linecount $=1$

altformb $=$.false.

rivindex $=0$

C

write $(*, *)$,

write $(*, *)$,

write $(*, *)$ ' Read Monolith File'

do 10 while(.not.endfile)

read(iunmn, 110, end $=99$ ) buffer(linecount)

if(mod(linecount, 100).eq.0) write $\left(*,{ }^{*}\right)$ ' At Monument File line \#',

C

$+\quad$ linecount

tembuf $=$ buffer(linecount)

C

if(linecount.eq.1) then

if(tembuf(1:2) .eq. 'RM') then

write $(*, *)$ ' Alternative Monolith File Format B'

altformb $=$.true.

endif

endif

C

if(altformb) then

read(tembuf(3:9), ${ }^{*}$ ) rivmaltb

read(tembuf(10:12),511) rivshorealtb

read(tembuf(12:49), ${ }^{*}$,err $\left.=999\right)$ noraltb, eastaltb, elevaltb

C

read(tembuf(50:56), ${ }^{*}$,err $\left.=999\right)$ stationaltb

rivmile $=$ rivmaltb $* 100$.

write(tembuf,500) noraltb, eastaltb, elevaltb, stationaltb

write(tembuf(57:61),'(i5)') rivmile

buffer(linecount) $=$ tembuf

else

read(tembuf(57:61), ${ }^{*}$, err $\left.=999\right)$ rivmile

endif 


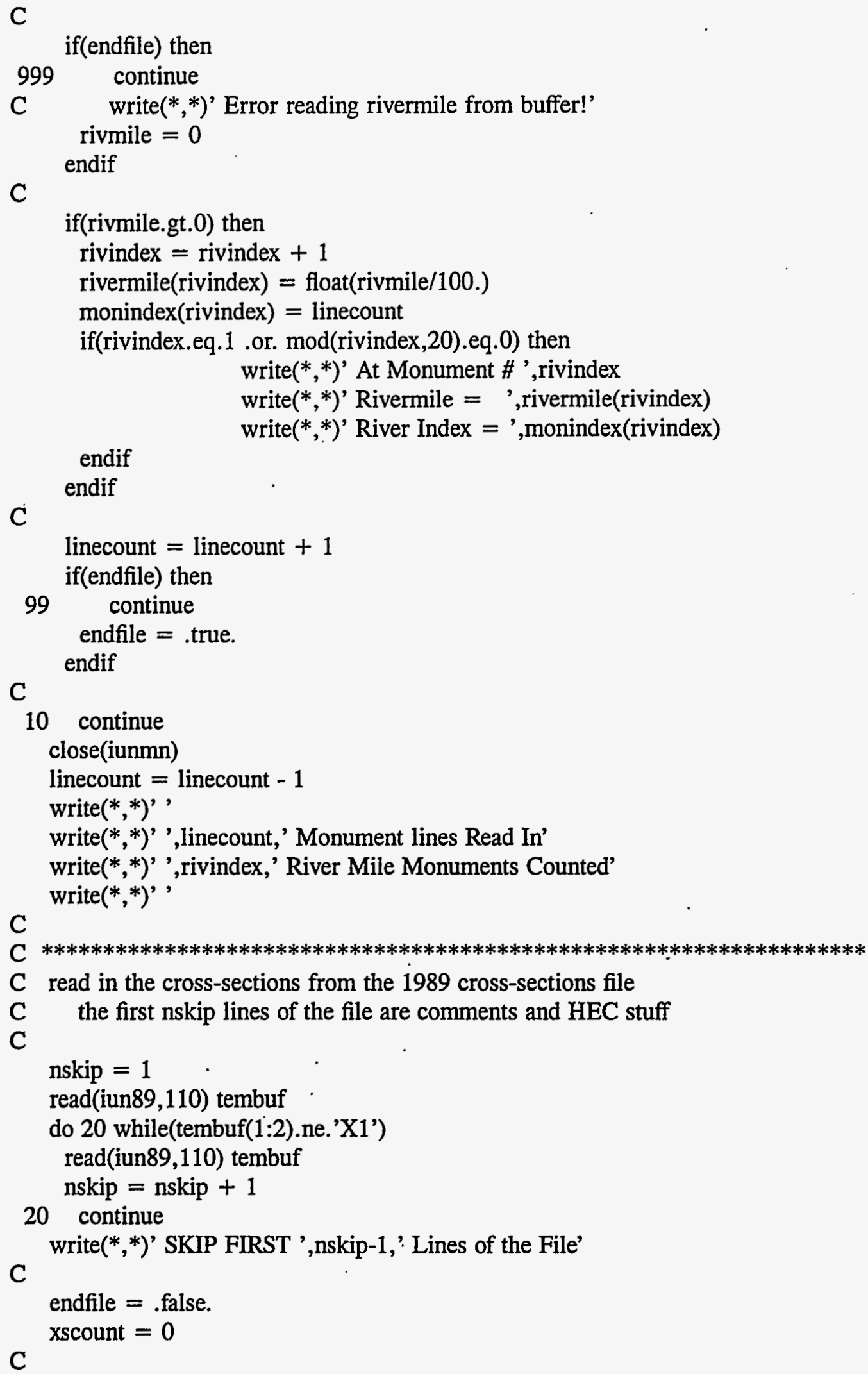


write $(*, *)^{\prime}$,

write $(*, *)$ ' Read Cross-Sections File'

xshead $=$ tembuf

do 30 while(.not. endfile)

779 if(xscount.gt.0) read(iun89,110,end =98) xshead

if(xshead(1:2).ne.' $X 1$ ') then

if(xshead(1:1).eq. '*') go to 779

write $(*, *)$ ' At XSECTION = ',xscount +1

write $(*, *)$ ' Header line incorrect'

write $(*, *)$ xshead

stop' CRASHH and BURN Babe!'

endif

read(xshead,300) xsrivermile, numstations, start_station,

$+\quad$ end_station

xscount $=$ xscount +1

if(xscount.eq. 1 .or. $\bmod (x s c o u n t, 25) . e q .0)$ then

write $(*, *)$ ' At Cross Section \#',xscount

write $(*, *)$ ' At Rivermile $=$ ',xsrivermile

write $(*, *)$ ' Number of Stations in Xsect $=$ ',numstations

endif

C

C the number of records to read for this cross section is the

C total number of stations divided by 5 stations per line.

C any extra stations are counted separately.

C

numrecs $=$ int(numstations $/ 5$ )

num_extra_stations $=$ numstations $-($ numrecs $* 5)$

C

C read in the values for the cross-section

C

station index $=1$

do $35 \overline{\mathrm{i}}=1$, numrecs

78 read(iun89,110) sectbuff(1:80)

if(sectbuff(1:2).ne.'GR') go to 78

C

C check for the B revision format of GR files with all

C five coordinate pairs on one line (instead of 2 lines)

C

if(sectbuff(80:80).eq.' ') then

read(iun89,110) sectbuff(75:154)

endif

$\mathrm{ib}=$ station_index

$\mathrm{ie}=\mathrm{ib}+5-1$

$\operatorname{read}\left(\operatorname{sectbuff}(3: 80),{ }^{*}\right)$ (station_elev(j),station(j),j=ib,ie)

35 continue

station_index $=$ ie +1

station_index $=$ station_index -1 


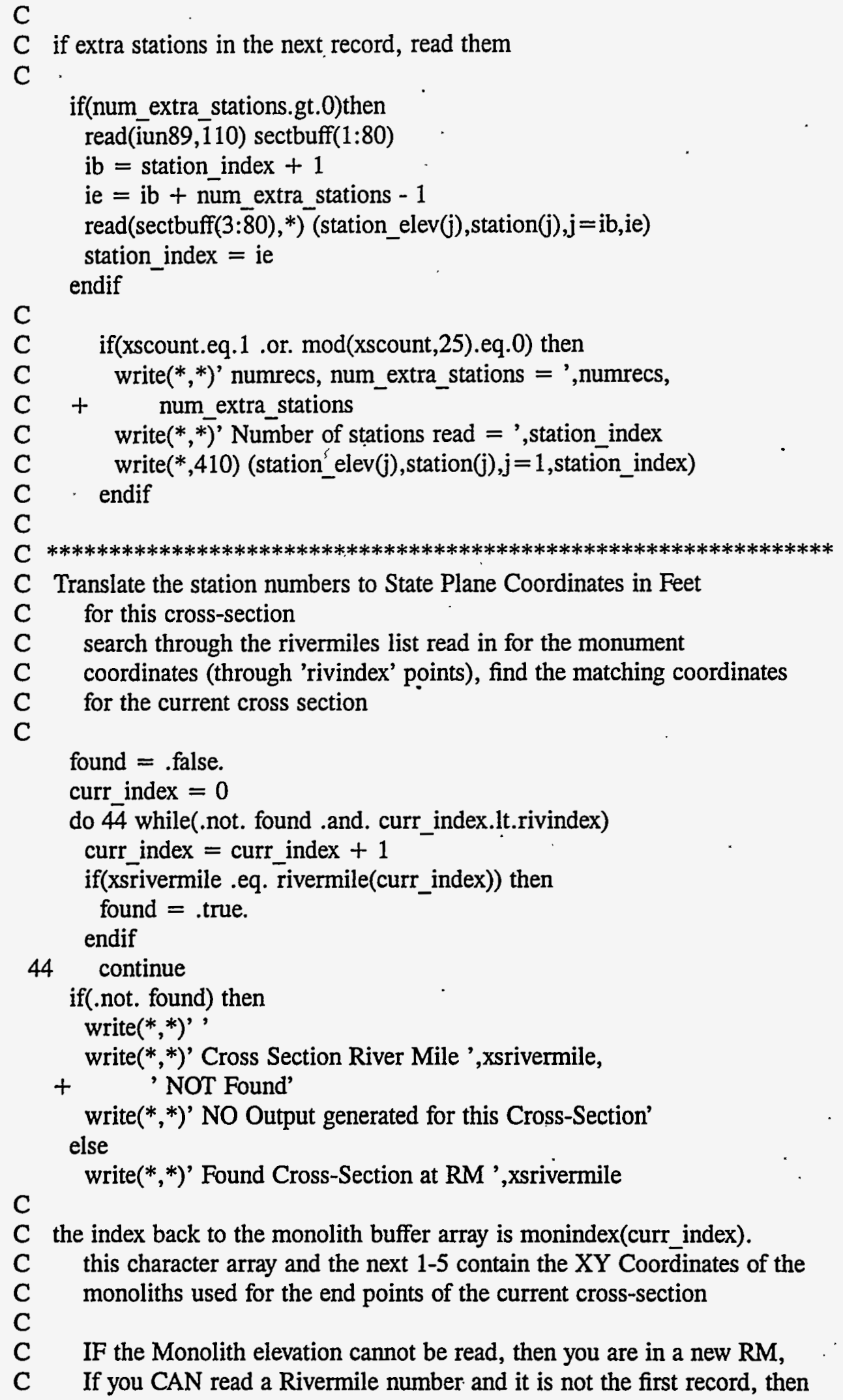


C you are at a new rivermile. IP is the number of monolith entries for

C this rivermile after the current index counter.

C NOTE: Monitor file is Nothings THEN Eastings ( $\mathrm{Y}, \mathrm{X})$

C

same_rm $=$.true.

ic $=$ monindex(curr_index)

ip $=1$

do 55 while(same $\mathrm{rm}$ )

tembuf $=$ buffer(ic)

read(tembuf,500,err =598) mony(ip), monx(ip),

$+\quad$ monelev(ip), monstation(ip)

if(monelev(ip).le.0.0) go to 598

ip $=\mathrm{ip}+1$

read(tembuf(57:61), ${ }^{*}$, err $\left.=599\right) \mathrm{rm}$

write $(*, *)^{\prime} \mathrm{RM}=$ ', $\mathrm{rm}$

if(ip.eq. 1) then

old $\mathrm{rm}=\mathrm{rm}$

else if(rm.eq.old_rm) then

endif

go to 599

if(ip.ne.2) then

$$
\text { ip }=\text { ip }-1
$$

same_rm $=$.false.

endif

$$
\text { go to } 599
$$

C

$$
\text { if(.not. same_rm) then }
$$

598 continue

$$
\text { ip }=\text { ip }-1
$$

endif

same_rm $=$.false.

C

599

if(.not. same_rm) then

C

endif continue

C

$$
\text { ic }=\text { ic }+1
$$

55

continue

write $(*, *)$ 'ip $=$,,ip

do $888 \mathrm{i}=1$,ip

write $(*, *) \operatorname{monx}(\mathrm{i})$, mony(i), monelev(i), monstation(i)

888 continue

C

C $* * * * * * * * * * * * * * * * * * * * * * * * * * * * * * * * * * * * * * * * * * * * * * * * * * * * * * * * * * * * * * * * * *$

C NOW we actually get to do some work on the data !

C 
C 1) Determine which 2 of the IP monoliths are closest to the ones

C used for this Cross-Section

$C$ 2) Use these 2 monoliths to interpolate the $X Y$ locations of all the

C points across the Cross-Section

C

if(ip.gt.2) then

maxdiff $=1.0 \mathrm{e}+8$

do $777 \mathrm{i}=1$,ip

diff $=$ abs(start_station - monstation(i))

if(diff .lt. maxdiff) then

maxdiff $=$ diff

ind $1=\mathrm{i}$

777

endif

C

continue

$$
\begin{aligned}
& \text { maxdiff }=1.0 \mathrm{e}+16 \\
& \text { do } 778 \mathrm{i}=1, \mathrm{ip}
\end{aligned}
$$

diff = abs(end_station - monstation(i))

if(diff .lt. maxdiff) then

$$
\text { maxdiff }=\text { diff }
$$$$
\text { ind } 2=\mathrm{i}
$$

$$
\text { endif }
$$

778 continue

C

$$
\text { else }
$$

C

C if only two monolith points, assume are in correct order (?)

C

$$
\begin{aligned}
\text { ind } 1 & =1 \\
\text { ind } 2 & =2 \\
\text { endif } &
\end{aligned}
$$

C

$$
\begin{aligned}
& \text { xstart }=\text { monx(ind1) } \\
& \text { ystart }=\text { mony(ind1) } \\
& \text { zstart }=\text { monelev(ind1) } \\
& \text { start_dist }=\text { monstation(ind1) } \\
& \text { xend }=\text { monx(ind2) } \\
& \text { yend }=\text { mony(ind2) } \\
& \text { zend }=\text { monelev(ind2) }
\end{aligned}
$$

if(xsrivermile.eq.103.57.or. xsrivermile.eq.104.26) then

write $(*, *)$ ' xsrivermile $=$ ',xsrivermile

write $(*, *)$ ' ip, ind1, ind2 = ', ip,ind1, ind2

write $(*, *)$ 'xstart,ystart,zstart,start_dist $=$ '

$+\quad$,xstart,ystart,zstart,start_dist

write $(*, *)$ ' xend,yend,zend $=$ ', xend,yend,zend

endif 
C For each of the points of the Cross-Section, calculate the XY

C position of the point.

C do $666 \mathrm{i}=1$,numstations call interp_xy(xstart,ystart,xend,yend,start_dist,

$+\quad$ station(i),xout, yout)

write(oun,600) xout,yout,station_elev(i),xsrivermile

666 continue

C

endif

C

if(endfile) then

98 continue

endfile $=$.true

endif

$\mathrm{C}$

30 continue

C

close(iun89)

C

close(oun)

C $* * * * * * * * * * * * * * * * * * * * * * * * * * *$

C Format Statements

C

100 format(a40)

110 format(a80)

300 format $(2 \mathrm{x}, \mathrm{f6} .2,4 \mathrm{x}, \mathrm{f5} .0,2 \mathrm{f} 8.1)$

410 format $(2 \mathrm{x}, 5(\mathrm{f} 6.1,2 \mathrm{x}, \mathrm{f6} .1,2 \mathrm{x}), /)$

500 format(f10.2,5x,f10.2,3x,f7.2,26x,f8.2)

511 format(a3)

515 format(f7.2)

600 format $(1 \mathrm{x}, 2 \mathrm{f} 12.2, \mathrm{f} 12.4, \mathrm{f12} .4)$

C

stop

C

end

C $* * * * * * * * * * * * * * * * * * * * * * * * * * * * * * * * * * * * * * * * * * * * * * * * * * * * * * * * * * * * * * * * * * * * * * *$

C

subroutine interp_xy(x1,y1,x2,y2,d1, distin, xout,yout)

C

implicit none

double precision $\quad \mathrm{x} 1, \mathrm{y} 1, \mathrm{x} 2, \mathrm{y} 2, \mathrm{~d} 1$, xout, yout

real distin

C

real line_dist, delx,dely, deldist, prop

line_dist $=$ distin - d1

$\operatorname{del} \bar{x}=\mathrm{x} 1-\mathrm{x} 2$ 


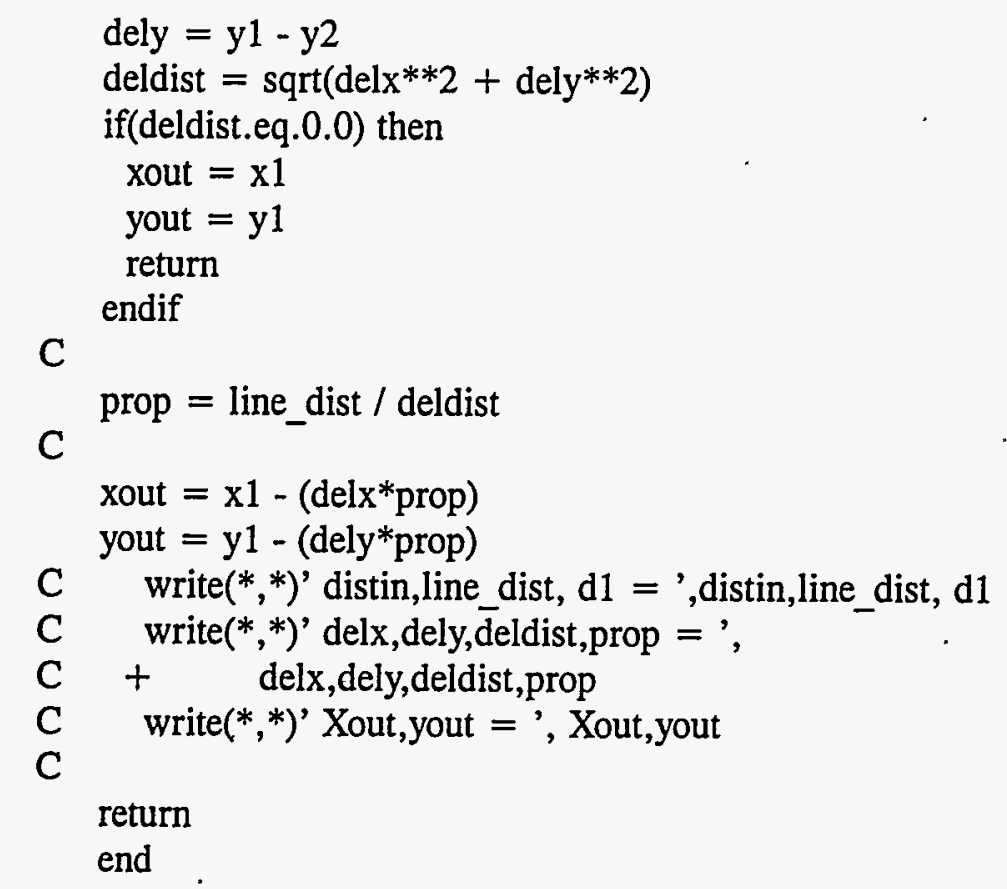



Appendix C

Example from USCOE HEC-2 Modeling Runs 



\section{Appendix C}

\section{Example from USCOE HEC-2 Modeling Runs}

* SNAKE RIVER SET UP FOR NATURAL CONDITIONS- DAMS NOT IN PLACE

* SECTIONS FROM MOUTH TO ICE HARBOR SURVEYED

* SECTIONS FROM ICE HARBOR TO RM 101 BASED ON 1935-57 RIVER SOUNDING

* AND TOPOGRAPHY MAPS

* SECTIONS FORM 101.87 TO LOWER GRANITE DAM FROM 1987 LITTLE GOOSE SURV.

* SECTIONS FROM LOWER GRANITE DAM UPSTREAM: 1989 HYDROGRAPHIC SURVEYS.

T1 SNAKE RIVER FROM COLUMBIA-SNAKE CONFLUENCE TO SNAKE RIVER MILE 148.83

$\mathrm{T} 2$ 21 JUNE 91

T3

$\begin{array}{llllllllll}\mathrm{J} 1 & -10 & 2 & 0 & 0 & 0 & 0 & 0 & 0 & 337.0\end{array}$

0

$\begin{array}{llll}\mathrm{J} 2 & -1 & 0 & -1\end{array}$

$\begin{array}{llllllllll}\mathrm{J} 3 & 38 & 43 & 1 & 2 & 3 & 11 & 12 & 8 & 42\end{array}$

$\begin{array}{llllllllll}39 & & & & & & & & & \\ \mathrm{~J} 3 & 0 & 38 & 55 & 26 & 56 & 13 & 14 & 15 & 25\end{array}$

J5 $\quad-10 \quad-10$

QT 8 20000. 25000. 30000. 35000. 40000. 45000. 50000. 55000.

NC $0.040 \quad 0.040 \quad 0.028 \quad 0.10 \quad 0.30$

NC $\quad .028$

$\begin{array}{llllllll}\mathrm{X} 1 & 0.1 & 21 & 2690 & 5928 & 0000 & 0000 & 0000\end{array}$

$\begin{array}{lllllllll}\text { GR } 347.6 & 2666 & 340.4 & 2690 & 336.8 & 2962 & 330.3 & 3113 & 322.8\end{array}$

3200

$\begin{array}{lllllllll}\text { GR } 317.6 & 3400 & 317.1 & 3601 & 315.0 & 3779 & 292.5 & 4001 & 287.5\end{array}$

4200

$\begin{array}{lllllllll}\text { GR } 285.1 & 4399 & 288.3 & 4600 & 291.3 & 4800 & 299.4 & 5000 & 305.4\end{array}$

5200

$\begin{array}{lllllllll}\text { GR } 308.1 & 5400 & 307.6 & 5600 & 308.9 & 5775 & 321.3 & 5870 & 340.3\end{array}$ 5928

GR $345.2 \quad 5936$

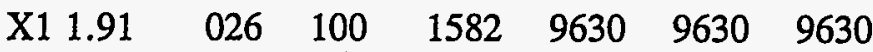

$\begin{array}{lllllllll}\text { GR } 340.0 & 0 & 336.0 & 20 & 337.4 & 22 & 336.5 & 60 & 334.3\end{array}$ 
100

$\begin{array}{lllllllll}\text { GR } 328.7 & 118 & 329.0 & 138 & 330.8 & 154 & 329.0 & 165 & 327.0\end{array}$ 218

$\begin{array}{lllllllll}\text { GR } 318.2 & 380 & 316.2 & 550 & 317.0 & 700 & 318.0 & 800 & 317.3\end{array}$ 946

$\begin{array}{lllllllll}\text { GR } 315.0 & 1000 & 305.0 & 1125 & 302.5 & 1184 & 301.5 & 1363 & 306.5\end{array}$ 1406

$\begin{array}{lllllllll}\text { GR } 305.6 & 1427 & 321: 2 & 1580 & 337.0 & 1582 & 340.0 & 1631 & 343.0\end{array}$ 1640

GR 347.31690

$\begin{array}{lllllll}\mathrm{X} 12.86 & 027 & 80 & 1815 & 5100 & 5500 & 5570\end{array}$

$\begin{array}{lllllllll}\text { GR 351.2 } & 0 & 349.0 & 10 & 341.3 & 20 & 335.2 & 80 & 324.0\end{array}$

100

$\begin{array}{lllllllll}\text { GR } 312.0 & 230 & 311.8 & 477 & 309.0 & 611 & 309.0 & 690 & 311.0\end{array}$

720

$\begin{array}{lllllllll}\text { GR } 324.8 & 830 & 324.8 & 848 & 322.1 & .880 & 322.2 & 912 & 327.5\end{array}$ 1092

$\begin{array}{lllllllll}\text { GR } 327.2 & 1120 & 322.0 & 1157 & 322.6 & 1200 & 330.0 & 1322 & 326.0\end{array}$ 1375

$\begin{array}{lllllllll}\text { GR } 329.0 & 1400 & 330.3 & 1437 & 325.7 & 1531 & 317.8 & 1604 & 317.2\end{array}$ 1700

GR $323.2 \quad 1762 \quad 340.0 \quad 1815$

$\begin{array}{lllllll}\text { X1 } 3.84 & 029 & 82 & 2822 & 5200 & 5400 & 5240 .\end{array}$

$\begin{array}{lllllllll}\text { GR } 344.4 & 0 & 336.0 & 40 & 325.0 & 82 & 317.7 & 243 & 321.0\end{array}$

375

$\begin{array}{lllllllll}\text { GR } 310.0 & 520 & 310.0 & 577 & 319.8 & 676 & 322.2 & 800 & 322.2\end{array}$ 1000

$\begin{array}{lllllllll}\text { GR } 323.3 & 1088 & 335.4 & 1140 & \cdot 337.8 & 1192 & 336.0 & 1243 & 339.0\end{array}$ 1308

$\begin{array}{lllllllll}\text { GR } 336.9 & 1380 & 332.0 & 1427 & 339.0 & 1578 & 335.8 & 1636 & 341.0\end{array}$ 1705

$\begin{array}{lllllllll}\text { GR } 341.7 & 1848 & 343.4 & 1897 & 342.0 & 2206 & 335.2 & 2332 & 317.8\end{array}$ 2632

$\begin{array}{llllllll}\text { GR } 318.0 & 2762 & 319.9 & 2790 & 331.8 & 2822 & 338.5 & 2903\end{array}$

$\begin{array}{llllllll}\mathrm{X} 1 & 5.09 & 21 & 292 & 1920 & 6700 & 7500 & 7190\end{array}$

$\begin{array}{lllllllll}\text { GR } 338.5 & 0 & 337.0 & 62 & 334.0 & 227 & 336.8 & 292 & 327.5\end{array}$

380

$\begin{array}{lllllllll}\text { GR } 321.8 & 575 & 326.0 & 620 & 321.4 & 730 & 320.0 & 792 & 322.0\end{array}$ 802

$\begin{array}{lllllllll}\text { GR } 321.5 & 835 & 319.0 & 945 & 320.8 & 972 & 320.0 & 1021 & 329.0\end{array}$ 1078

$\begin{array}{lllllllll}\text { GR } 327.8 & 1102 & 327.0 & 1343 & 328.5 & 1780 & 334.3 & 1857 & 345.8\end{array}$ 1920

GR $356.7 \quad 1932$

$\begin{array}{llllllll}\mathrm{X} 1 & 5.84 & 27 & 67 & 1379 & 4200 & 4500 & 4370\end{array}$

$\begin{array}{lllllllll}\text { GR } 338.4 & 0 & 333.3 & 67 & 332.5 & 278 & 334 & 415 & 328.7\end{array}$

592 
$\begin{array}{lllllllll}\text { GR } 329.2 & 641 & 328.7 & 662 & 329.8 & 680 & 323.4 & 690 & 318.6\end{array}$

703

$\begin{array}{lllllllll}\text { GR } 313.5 & 748 & 313.8 & 778 & 318.4 & 800 & 329.0 & 817 & 331.3\end{array}$

842

$\begin{array}{lllllllll}\text { GR } 326.9 & 867 & 332.0 & 912 & 330.0 & 930 & 332.2 & 1083 & 331.2\end{array}$

1097

$\begin{array}{lllllllll}\text { GR } 321.8 & 1109 & 320.8 & 1119 & 320.8 & 1177 & 320.0 & 1350 & 338.5\end{array}$

1379

$\begin{array}{llll}\text { GR } 347.5 & 1427 & 352.5 & 1440\end{array}$

$\begin{array}{llllllll}X 1 & 6.15 & 16 & 0 & 1586 & 2350 & 2350 & 2350\end{array}$

$\begin{array}{lllllllll}\text { GR } 340.1 & 0 & 336.2 & 20 & 331.7 & 130 & 327.7 & 313 & 327.7\end{array}$

438

$\begin{array}{lllllllll}\text { GR } 330.0 & 600 & 333.3 & 878 & 333.5 & 1110 & 332.8 & 1195 & 322.0\end{array}$

1253

$\begin{array}{lllllllll}\text { GR } 322.8 & 1285 & 320.0 & 1453 & 323.2 & 1526 & 335.5 & 1547 & 340.3\end{array}$

1586

GR $355.9 \quad 1627$

$\begin{array}{llllllll}\mathrm{X} 1 & 6.79 & 31 & 0 & 1418 & 2790 & 2790 & 2770\end{array}$

$\begin{array}{lllllllll}\text { GR } 340.4 & 0 & 336.7 & 20 & 328.8 & 167 & 327.0 & 180 & 327.8\end{array}$

220

$\begin{array}{lllllllll}\text { GR } 331.8 & 233 & 330.0 & 305 & 331.9 & 355 & 331.2 & 380 & 330.0\end{array}$

500

$\begin{array}{lllllllll}\text { GR } 329.6 & 661 & 332.0 & 706 & 327.0 & 715 & 331.5 & 762 & 332.0\end{array}$

870

$\begin{array}{lllllllll}\text { GR } 325.8 & 887 & 326.0 & 915 & 329.0 & 922 & 329.0 & 932 & 326.7\end{array}$

937

$\begin{array}{lllllllll}\text { GR } 326.0 & 965 & 330.3 & 992 & 328.3 & 1087 & 322.4 & 1145 & 321.0\end{array}$

1360.

$\begin{array}{lllllllll}\text { GR } 323.0 & 1387 & 336.7 & 1405 & 338.0 & 1418 & 336.0 & 1425 & 349.0\end{array}$

1470

GR $361.0 \quad 1485$

$\begin{array}{llllllll}\mathrm{X} 1 & 7.17 & 18 & 720 & 2200 & 730 & 730 & 730\end{array}$

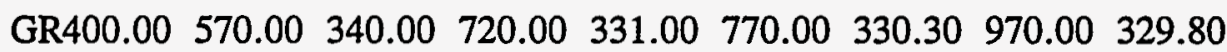

1170.00

GR329.10 $1320.00 \quad 324.601420 .00 \quad 322.201520 .00 \quad 330.00 \quad 1800.00 \quad 328.00$

1885.00

GR318.00 1912.00 318.20 1995.00 320.00 2012.00 323.00 2164.00 337.00

2190.00

GR342.002200.00 350.002320.00 360.003000.00

$\begin{array}{llllllll}\mathrm{X} 1 & 7.27 & 24 & 670 & 2200 & 530 & 530 & 530\end{array}$

GR400.00 $520.00 \quad 342.00 \quad 670.00 \quad 332.00 \quad 700.00 \quad 330.90 \quad 730.00 \quad 329.00$

800.00

GR330.60 $970.00 \quad 328.401070 .00 \quad 329.70 \quad 1220.00 \quad 329.00 \cdot 1270.00 \quad 325.80$ 1420.00

GR326.10 $1470.00 \quad 326.101630 .00 \quad 328.00 \quad 1720.00 \quad 330.00 \quad 1780.00 \quad 328.00$ 1885.00

GR318.00 1912.00 318.20 1995.00 320.00 2012.00 323.00 2164.00 337.00 
2176.00

GR341.00 2190.00 342.002200.00 350.00 2320.00 360.00 3000.00

$\begin{array}{llllllll}\mathrm{X} 1 & 7.46 & 19 & 670 & 2200 & 1000 & 1000 & 1000\end{array}$

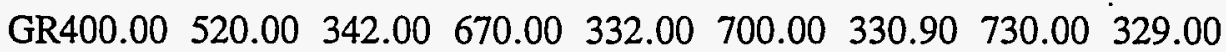

800.00

GR335.10 $1280.00 \quad 333.301380 .00 \quad 331.00 \quad 1500.00 \quad 329.30 \quad 1650.00 \quad 330.00$ 1800.00

GR328.00 $1885.00 \quad 318.001912 .00 \quad 318.201995 .00 \quad 320.002012 .00 \quad 323.00$

2164.00

GR337.00 2176.00 342.002200.00 $350.002320 .00 \quad 360.003000 .00$

$\begin{array}{llllllll}\mathrm{X} 1 & 7.84 & 25 & 570 & 2170 & 2000 & 2000 & 2000\end{array}$

GR360.00 $420.00 \quad 350.00 \quad 540.00 \quad 340.00 \quad 570.00 \quad 336.40 \quad 600.00 \quad 334.00$

660.00

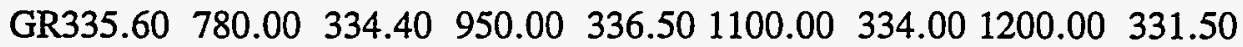

1300.00

GR335.00 $1400.00334 .001600 .00 \quad 336.001700 .00 \quad 335.00 \quad 1770.00 \quad 330.00$ 1830.00

GR327.50 1862.00 315.50 1896.00 316.00 2019.00 317.00 2052.00 316.00 2150.00

GR330.00 2160.00 $335.002170 .00 \quad 340.002180 .00 \quad 350.002200 .00 \quad 400.00$ 2975.00

$\begin{array}{llllllll}\mathrm{X} 1 & 8.03 & 27 & 580 & 2190 & 1000 & 1000 & 1000\end{array}$

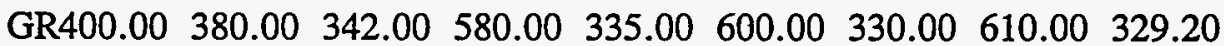

700.00

GR330.00 $770.00 \quad 335.00 \quad 810.00 \quad 342.00 \quad 860.00 \quad 348.50 \quad 1000.00 \quad 342.00$

1110.00

GR336.20 $1170.00331 .001270 .00331 .801350 .00 \quad 331.001460 .00332 .00$ 1570.00

GR334.40 $1660.00 \quad 333.401820 .00 \quad 332.501852 .00 \quad 315.201896 .00 \quad 315.00$ 1950.00

GR316.50 $1995.00315 .402054 .00 \quad 316.202145 .00 \quad 328.002166 .00 \quad 342.00$ 2190.00

GR350.00 2220.00 400.002500 .00

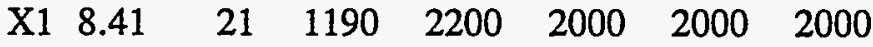

GR400.00 $260.00 \quad 341.80 \quad 410.00 \quad 335.00 \quad 470.00 \quad 334.00 \quad 600.00 \quad 335.00$

670.00

GR340.00 $720.00345 .001000 .00 \quad 340.001190 .00 \quad 335.001410 .00 \quad 330.00$

1640.00

GR329.30 $1700.00331 .901800 .00 \quad 333.201831 .00 \quad 318.401879 .00 \quad 316.50$

1935.00

GR318.00 2002.00 318.002162.00 333.00 2180.00 $340.002200 .00 \quad 350.00$

2220.00

GR400.00 2500.00

$\begin{array}{llllllll}\mathrm{X} 1 & 8.60 & 15 & 850 & 2184 & 1000 & 1000 & 1000\end{array}$

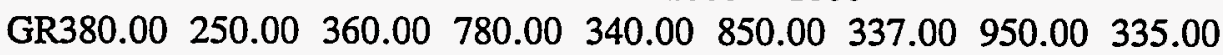

1150.00

GR334.30 1550.00 $330.001700 .00 \quad 328.001860 .00 \quad 316.201884 .00 \quad 318.30$ 
1990.00

- GR316.002068.00 318.002165.00 341.602184.00 350.002250 .00400 .00 2500.00

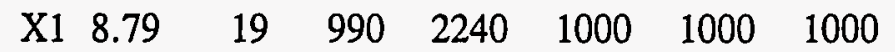

$\begin{array}{lllllll}\text { GR380.00 } & 740.00 \quad 370.00 \quad 910.00 & 342.60 & 990.00 \quad 340.00 & 1010.00 & 330.00\end{array}$ 1180.00

GR330.00 1270.00 335.00 1380.00 340.00 1470.00 343.00 $1570.00 \quad 340.00$ 1670.00

GR335.00 $1750.00330 .001810 .00 \quad 330.001831 .00 \quad 315.001883 .00 \quad 318.90$ 2130.00

GR332.00 2164.00 335.002190.00 350.002240.00 400.00 2320.00

$\begin{array}{llllllll}\mathrm{X} 1 & 8.97 & 18 & 1040 & 2260 & 1000 & 1000 & 1000\end{array}$

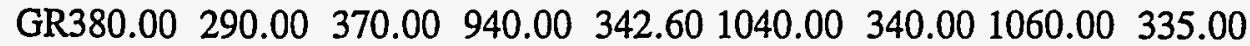
1150.00

GR330.00 $1240.00 \quad 330.001320 .00 \quad 335.001400 .00 \quad 335.201650 .00332 .00$ 1827.00

GR316.50 1892.00 316.602132.00 325.50 2152.00 330.00 2180.00 335.00 2200.00

GR342.80 2260.00 350.002300.00 400.00 2380.00

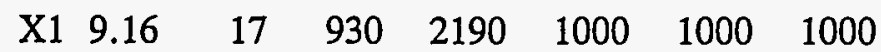

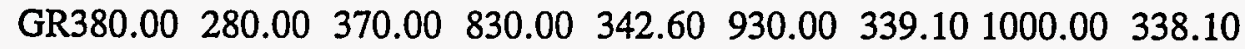
1050.00

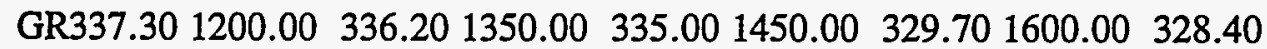
1800.00

GR325.00 1866.00 $317.601878 .00318 .002114 .00 \quad 326.002137 .00 \quad 343.60$ 2190.00

GR350.002250.00 400.002380 .00

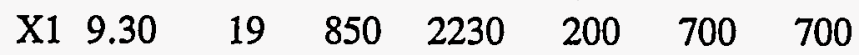

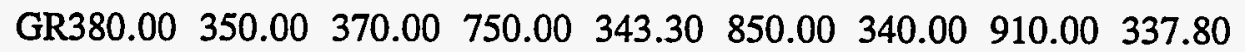
980.00

GR337.60 1200.00 335.00 1369.00 330.00 1380.00 325.00 1400.00 323.00 1450.00

GR320.90 $1530.00 \quad 322.701570 .00 \quad 322.801730 .00 \quad 320.001800 .00326 .00$ 1900.00

GR325.00 2180.00 343.602230.00 350.002300.00 400.002500 .00

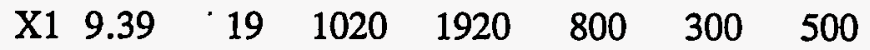

GR380.00 620.00 $370.00 \quad 920.00 \quad 343.30 \quad 1020.00 \quad 340.00 \quad 1030.00 \quad 335.00$ 1170.00

GR330.00 1310.00 $330.001370 .00 \quad 325.001410 .00 \quad 320.001450 .00315 .00$ 1530.00

GR313.00 $1550.00313 .601600 .00315 .001700 .00 \quad 320.001750 .00325 .00$ 1780.00

GR325.10 $1920.00 \quad 350.001930 .00 \quad 350.002300 .00 \quad 400.002500 .00$

$\begin{array}{llllllll}\mathrm{X} 1 & 9.69 & 41 & 0 & 2430 & 1584 & 1584 & 1584\end{array}$

$\begin{array}{llllllllll}\text { GR } & 425 & 0 & 400 & 60 & 395 & 83 & 390 & 105 & 385\end{array}$

120

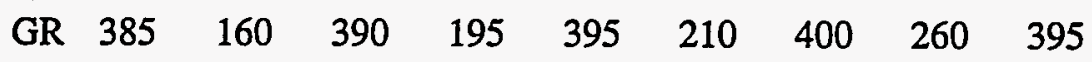


300

$\begin{array}{llllllllll}\text { GR } & 390 & 325 & 385 & 338 & 380 & 358 & 375 & 382 & 360\end{array}$

420

$\begin{array}{llllllllll}\text { GR } & 355 & 472 & 350 & 578 & 345 & 645 & 340 & 685 & 338.9\end{array}$

730

$\begin{array}{llllllllll}\text { GR } & 340 & 770 & 340 & 1130 & 338.9 & 1145 & 333 & 1272 & 330\end{array}$ 1400

$\begin{array}{lllllllll}\text { GR } 327.7 & 1590 & 330 & 1750 & 333 & 1780 & 338.9 & 1925 & 340\end{array}$

1960

$\begin{array}{llllllllll}\text { GR } & 350 & 1975 & 375 & 2040 & 400 & 2100 & 405 & 2128 & 410\end{array}$

2200

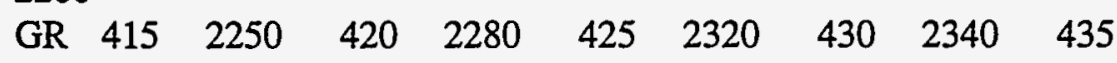

2372

GR $440 \quad 2430$

* ICE HARBOR DAM. RM 9.7

$\begin{array}{llllllll}\mathrm{X} 1 & 9.7 & 41 & 0 & 2430 & 1 & 1 & 1\end{array}$

$\begin{array}{llllllllll}\mathrm{GR} & 425 & 0 & 400 & 60 & 395 & 83 & 390 & 105 & 385\end{array}$

120

$\begin{array}{llllllllll}\text { GR } & 385 & 160 & 390 & 195 & 395 & 210 & 400 & 260 & 395\end{array}$

$\begin{array}{llllllllll}300 & & & & & & & & \\ \text { GR } & 390 & 325 & 385 & 338 & 380 & 358 & 375 & 382 & 360\end{array}$

$\begin{array}{llllllllll}420 & & & & & & & & & \\ \text { GR } & 355 & 472 & 350 & 578 & 345 & 645 & 340 & 685 & 338.9\end{array}$

$\begin{array}{llllllllll}730 & & & & & & & & & \\ \mathrm{GR} & 340 & 770 & 340 & 1130 & 338.9 & 1145 & 333 & 1272 & 330\end{array}$

1400

$\begin{array}{lllllllll}\text { GR } 327.7 & 1590 & 330 & 1750 & 333 & 1780 & 338.9 & 1925 & 340\end{array}$

1960

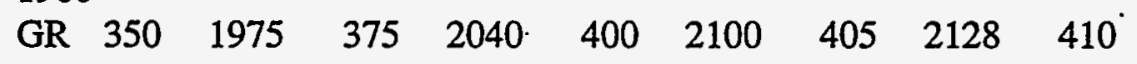

2200

$\begin{array}{llllllllll}\text { GR } & 415 & 2250 & 420 & 2280 & 425 & 2320 & 430 & 2340 & 435\end{array}$

2372

GR $440 \quad 2430$

$\begin{array}{lllll}\text { NC } 0.029 & 0.029 & 0.029 & 0.1 & 0.3\end{array}$

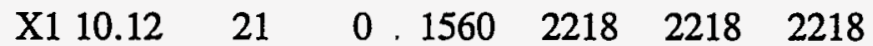

$\begin{array}{llllllllll}\text { GR } & 425 & 0 & 400 & 100 & 375 & 170 & 350 & .190 & 345\end{array}$

195

$\begin{array}{llllllllll}\text { GR } & 340 & 328 & 339 & 340 & 333 & 390 & 333 & 580 & 330\end{array}$

620

$\begin{array}{llllllllll}\text { GR } & 329 & 680 & 327 & 750 & 330 & 790 & 333 & 805 & 333\end{array}$

925

$\begin{array}{llllllllll}\text { GR } & 339 & 1315 & 340 & 1350 & 350 & 1370 & 375 & 1455 & 400\end{array}$

1520

GR $425 \quad 1560$

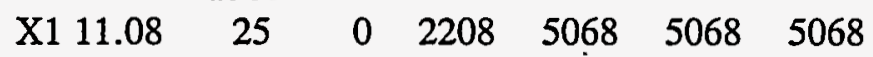

$\begin{array}{llllllllll}\text { GR } & 425 & 0 & 400 & 37 & 375 & 75 & 355 & 105 & 350\end{array}$

130 
$\begin{array}{llllllllll}\text { GR } & 345 & 158 & 342.4 & 190 & 337.2 & 290 & 342.4 & 470 & 345\end{array}$

505

$\begin{array}{llllllllll}\text { GR } & 345 & 815 & 342.4 & 890 & 340.2 & 1020 & 341.1 & 1130 & 333.7\end{array}$ 1270

$\begin{array}{lllllllll}\text { GR } 342.4 & 1670 & 345 & 1700 & 350 & 1710 & 375 & 1785 & 400\end{array}$ 1860

$\begin{array}{llllllllll}\text { GR } & 425 & 1878 & 435 & 1890 & 440 & 1970 & 445 & 2060 & 450\end{array}$ 2208

$\begin{array}{llllllll}X 1 & 11.9 & 34 & 0 & 1825 & 4330 & 4330 & 4330\end{array}$

$\begin{array}{llllllllll}\text { GR } & 425 & 0 & 415 & 82 & 410 & 135 & 400 & 200 & 390\end{array}$

282

$\begin{array}{llllllllll}\text { GR } & 380 & 352 & 375 & 375 & 370 & 408 & 365 & 430 & 360\end{array}$

470

$\begin{array}{llllllllll}\text { GR } & 355 & 497 & 350 & 530 & 345 & 557 & 340 & 608 & 339\end{array}$

$\begin{array}{llllllllll}640 & & & & & & & & \\ \text { GR } & 335 & 698 & 334 & 724 & 335 & 755 & 336 & 810 & 337\end{array}$

$\begin{array}{llllllllll}880 & & & & & & & & & \\ \text { GR } & 338 & 1020 & 339 & 1053 & 339.6 & 1200 & 339 & 1410 & 339\end{array}$

$\begin{array}{llllllllll}1490 & & & & & & \cdot & & & \\ \text { GR } & 345 & 1608 & 350 & 1650 & 355 & 1670 & 360 & 1688 & 365\end{array}$

1695

$\begin{array}{lllllllll}\text { GR } & 375 & 1718 & 400 & 1768 & 425 & 1808 & 440 & 1825\end{array}$

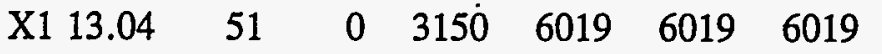

$\begin{array}{llllllllll}\text { GR } & 475 & 0 & 450 & 37 & 425 & 90 & 400 & 175 & 395\end{array}$

197

$\begin{array}{llllllllll}\text { GR } & 390 & 280 & 390 & 415 & 395 & 469 & 400 & 490 & 405\end{array}$

$\begin{array}{llllllllll}515 & & & & & & & & & \\ \text { GR } & 410 & 550 & 410 & 595 & 405 & 615 & 400 & 630 & 380\end{array}$

$\begin{array}{llllllllll}718 & & & & & & & & & \\ \text { GR } & 375 & 778 & 375 & 853 & 375 & 1050 & 365 & 1065 & 360\end{array}$

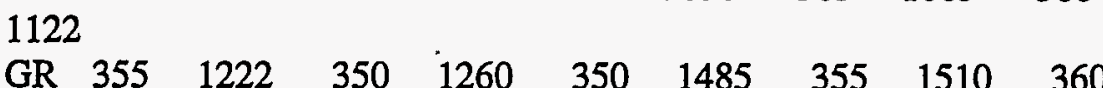

$\begin{array}{llllllll}1530 & & & & & & & \end{array}$

$\begin{array}{llllllllll}\text { GR } & 365 & 1570 & 365 & 1600 & 360 & 1620 & 355 & 1728 & 350\end{array}$

1778

$\begin{array}{lllllllll}\text { GR } 347.8 & 2022 & 343.5 & 2070 & 347.8 & 2120 & 350 & 2140 & 350\end{array}$ 2283

$\begin{array}{lllllllll}\text { GR } 347.8 & 2313 & 341.8 & 2408 & 338.8 & 2458 & 338.8 & 2573 & 341.8\end{array}$ 2578

$\begin{array}{lllllllll}\text { GR } 347.8 & 2635 & 350 & 2741 & 355 & 2760 & 360 & 2812 & 375\end{array}$

2865

$\begin{array}{llllllllll}\text { GR } & 400 & 2928 & 425 & 2983 & 430 & 3033 & 435 & 3084 & 445\end{array}$

3155

GR $450 \quad 3206$

$\begin{array}{llllllll}\mathrm{X} 1 & 14.1 & 43 & 0 & 3265 & 5597 & 5597 & 5597\end{array}$

$\begin{array}{llllllllll}\text { GR } & 440 & 0 & 430 & 42 & 425 & 75 & 420 & 132 & 415\end{array}$

162 


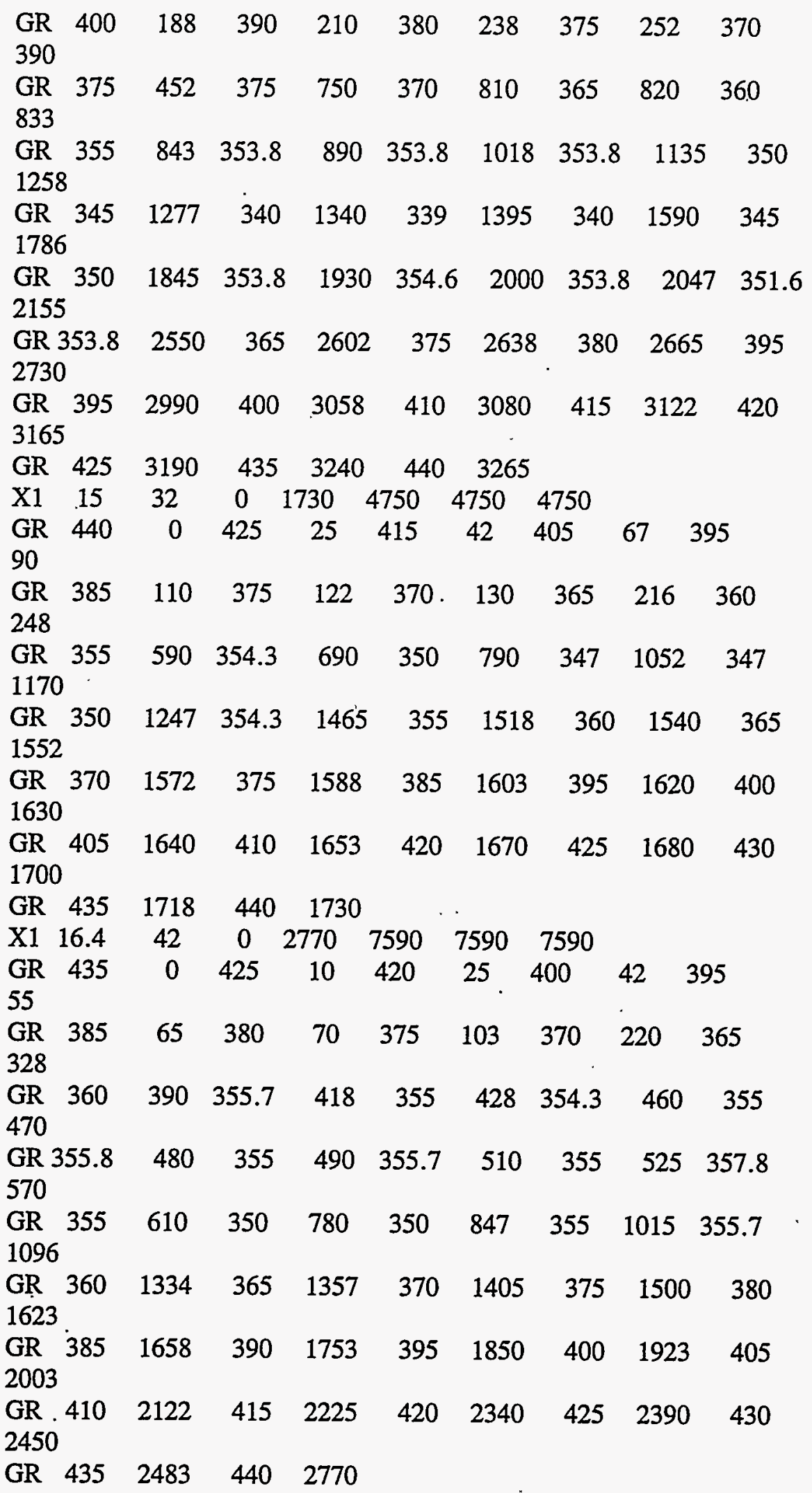




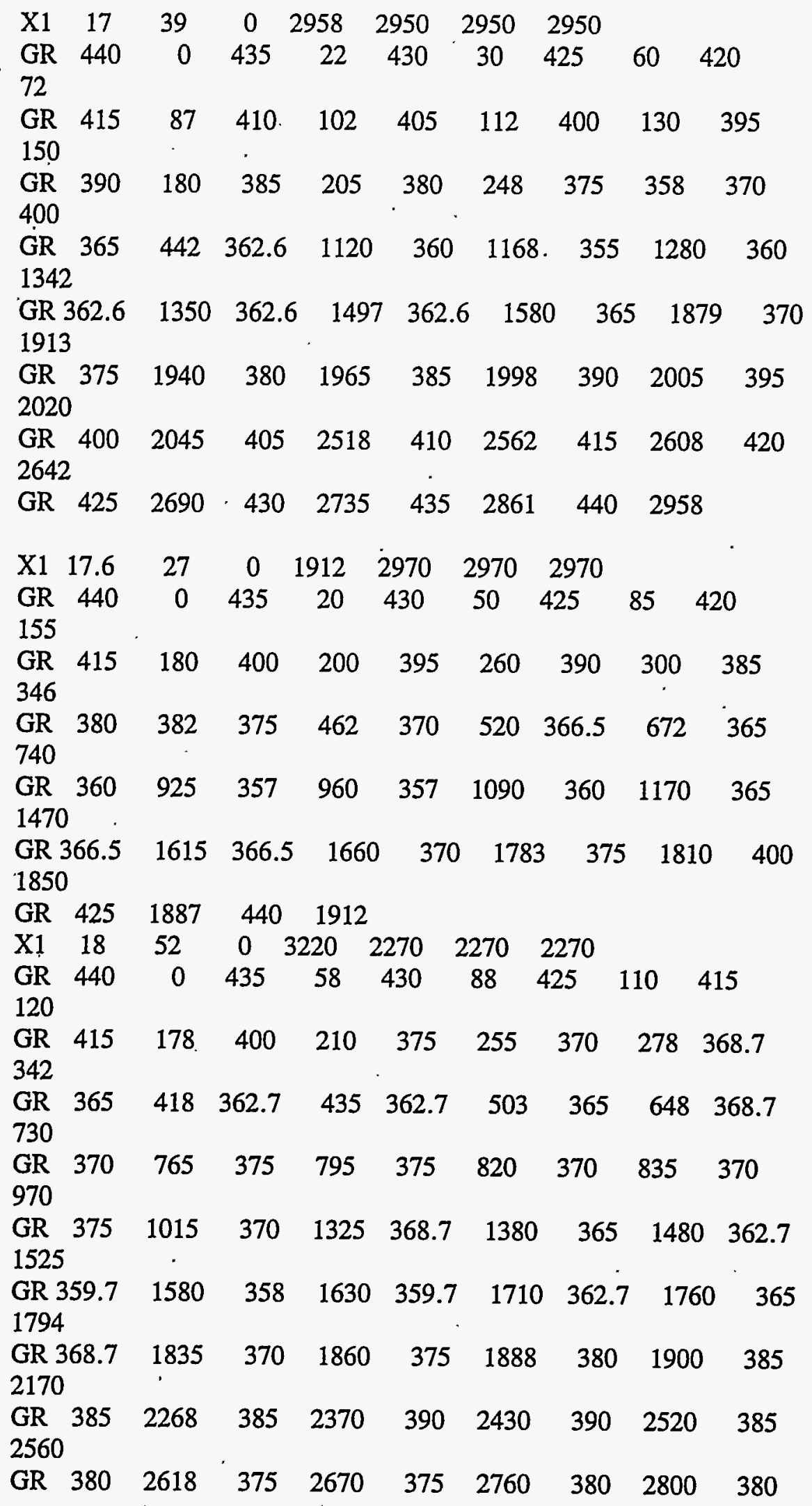


2880

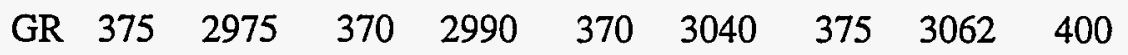
3138

GR $\quad 425 \quad 3170 \quad 440 \quad 3220$

$\begin{array}{llllllll}\mathrm{X} 1 & 18.3 & 39 & 0 & 4310 & 1777 & 1777 & 1777\end{array}$

$\begin{array}{llllllllll}\text { GR } & 440 & 0 & 435 & 20 & 430 & 33 & 425 & 44 & 420\end{array}$

50

$\begin{array}{llllllllll}\text { GR } & 415 & 60 & 410 & 80 & 405 & 95 & 400 & 105 & 390\end{array}$

120

$\begin{array}{llllllllll}\text { GR } & 385 & 130 & 375 & 145 & 370 & 155 & 369.1 & 240 & 367\end{array}$

312

$\begin{array}{llllllllll}\text { GR } & 367 & 470 & 369.1 & 585 & 370 & 608 & 375 & 750 & 375\end{array}$

1100

$\begin{array}{lllllllll}\text { GR } 369.1 & 1250 & 367 & 1280 & 365 & 1280 & 363 & 1292 & 363\end{array}$

1398

$\begin{array}{llllllllll}\text { GR } & 365 & 1730 & 367 & 1855 & 369.1 & 1870 & 370 & 1885 & 375\end{array}$

1900

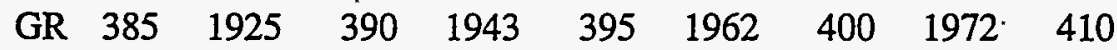

1990

$\begin{array}{lllllllll}\text { GR } & 415 & 2480 & 420 & 2870 & 425 & 3100 & 440 & 4310\end{array}$

$\begin{array}{llllllll}\mathrm{X} 1 & 18.5 & 45 & 0 & 3540 & 1010 & 1010 & 1010\end{array}$

$\begin{array}{llllllllll}\text { GR } & 440 & 0 & 435 & 35 & 430 & 68 & 425 & 112 & 420\end{array}$

150

$\begin{array}{llllllllll}\text { GR } & 415 & 180 & 410 & 210 & 405 & 245 & 400 & 258 & 390\end{array}$

278

$\begin{array}{llllllllll}\text { GR } & 380 & 300 & 375 & 310 & 370 & 345 & 369.3 & 390 & 367\end{array}$

440

$\begin{array}{llllllllll}\text { GR } & 367 & 490 & 370 & 568 & 375 & 705 & 376 & 730 & 375\end{array}$

750

$\begin{array}{llllllllll}\text { GR } & 370 & 828 & 367 & 895 & 365 & 1040 & 363 & 1100 & 363\end{array}$ 1128

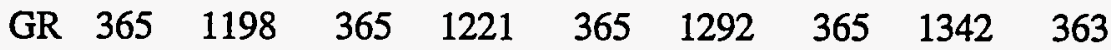

1410

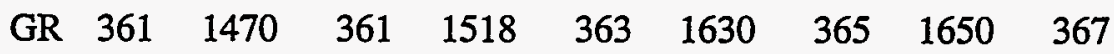

1662

$\begin{array}{lllllllll}\text { GR } 369.3 & 1670 & 370 & 1695 & 375 & 1710 & 385 & 1738 & 395\end{array}$

1773

$\begin{array}{llllllllll}\text { GR } & 400 & 1788 & 405 & 1852 & 410 & 1895 & 415 & 2375 & 420\end{array}$

3540

$\begin{array}{llllllll}\mathrm{X} 1 & 19.5 & 38 & 0 & 1790 & 5112 & 5112 & 5112\end{array}$

$\begin{array}{lllllllll}\text { GR } .440 & 0 & 435 & 19 & 430 & 30 & 425 & 50 & 420\end{array}$

75

$\begin{array}{llllllllll}\text { GR } & 415 & 92 & 410 & 120 & 405 & 178 & 400 & 186 & 395\end{array}$

193

$\begin{array}{llllllllll}\text { GR } & 390 & 210 & 385 & 253 & 380 & 261 & 375 & 270 & 369.5\end{array}$ 
292

$\begin{array}{llllllllll}\text { GR } & 365 & 425 & 360.5 & 489 & 360.5 & 503 & 360 & 549 & 360\end{array}$

665

$\begin{array}{llllllllll}\text { GR } & 355 & 790 & 352 & 839 & 355 & 1105 & 360 & 1230 & 365\end{array}$

1280

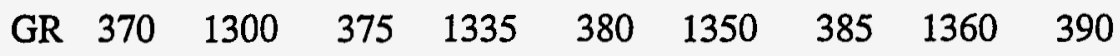

1430

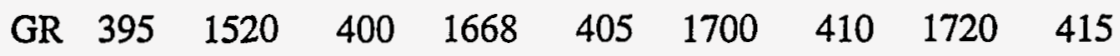

1740

$\begin{array}{lllllll}\text { GR } & 420 & 1760 & 430 & 1780 & 440 & 1790\end{array}$

$\begin{array}{llllllll}\mathrm{X} 1 & 19.7 & 25 & 0 & 872 & 1265 & 1265 & 1265\end{array}$

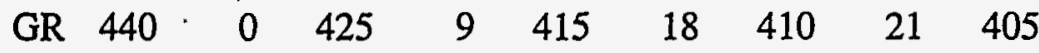

58

$\begin{array}{llllllllll}\text { GR } & 400 & 62 & 375 & 93 & 370 & 110 & 365 & 120 & 360\end{array}$

$\begin{array}{llllllllll}123 & & & & & & & & & \end{array}$

$\begin{array}{llllllllll}612 & & & & & & & & & \\ \text { GR } & 335 & 657 & 360 & 680 & 365 & 703 & 370 & 732 & 375\end{array}$

$\begin{array}{llllllllll}768 & & & & & & & & & \\ \text { GR } & 400 & 782 & 425 & 812 & 430 & 825 & 435 & 845 & 440\end{array}$

872

$\begin{array}{llllllll}X 1 & 20.52 & 24 & 0 & 715 & 3860 & 3860 & 3860\end{array}$

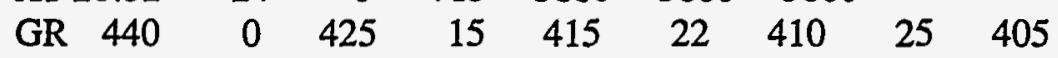

52

$\begin{array}{llllllllll}\text { GR } & 400 & 65 & 390 & 75 & 375 & 118 & 370 & 164 & 363.6\end{array}$

200

$\begin{array}{lllllllll}\text { GR } 360.6 & 213 & 355 & 223 & 328 & 250 & 320 & 340 & 321\end{array}$

435

$\begin{array}{llllllllll}\text { GR } & 341 & 490 & 354 & 535 & 360.6 & 550 & 363.6 & 563 & 370\end{array}$

595

$\begin{array}{lllllllll}\text { GR } & 375 & 620 & 400 & 672 & 425 & 690 & 440 & 715\end{array}$

$\begin{array}{llllllll}\mathrm{X} 1 & 21.2 & 21 & 0 & 1010 & 3630 & 3630 & 3630\end{array}$

$\begin{array}{llllllllll}\text { GR } & 440 & 0 & 425 & 20 & 400 & 55 & 375 & 94 & 370\end{array}$

115

$\begin{array}{llllllllll}\text { GR } & 365 & 210 & 360 & 287 & 355 & 318 & 350 & 368 & 350\end{array}$

$\begin{array}{llllllllll}423 & & & & & & & & & \\ \text { GR } & 355 & 502 & 360 & 662 & 365 & 768 & 370 & 827 & 375\end{array}$

$\begin{array}{llllllllll}858 & & & & & & & & & \\ \text { GR } & 380 & 880 & 400 & 918 & 410 & 952 & 425 & 975 & 430\end{array}$

990

GR $440 \quad 1010$

$\begin{array}{llllllll}\mathrm{X} 1 & 21.4 & 19 & 0 & 1350 & 850 & 850 & 850\end{array}$

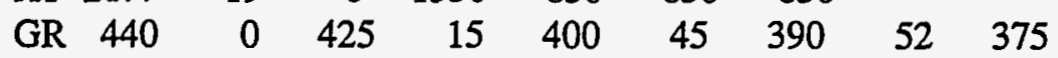

80

$\begin{array}{llllllllll}\text { GR } & 370 & 175 & 365 & 208 & 360 & 320 & 370 & 480 & 370\end{array}$

530

$\begin{array}{llllllllll}\text { GR } & 367 & 620 & 359 & 726 & 360 & 935 & 365 & 1025 & 370\end{array}$ 
1165

\begin{tabular}{lllllllll} 
GR & 375 & 1205 & 400 & 1250 & 425 & 1305 & 440 & 1350 \\
ET & 9.1 & & & & \multicolumn{4}{c}{768}
\end{tabular}

2783

$\begin{array}{llllllll}\mathrm{X} 1 & 22.2 & 49 & 768 & 2783 & 4540 & 4540 & 4540\end{array}$

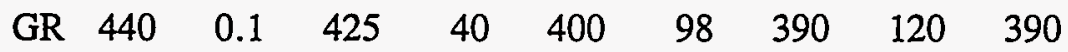

405

$\begin{array}{llllllllll}\text { GR } & 390 & 630 & 390 & 692 & 395 & 730 & 400 & 750 & 405\end{array}$

768

$\begin{array}{llllllllll}\text { GR } & 405 & 790 & 400 & 805 & 395 & 1000 & 390 & 1085 & 385\end{array}$

1088

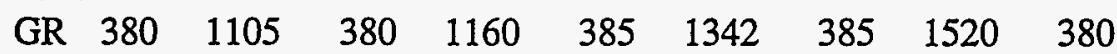

1688

$\begin{array}{llllllllll}\text { GR } & 375 & 1960 & 374.3 & 1980 & 370 & 2330 & 368.3 & 2370 & 366\end{array}$

2545

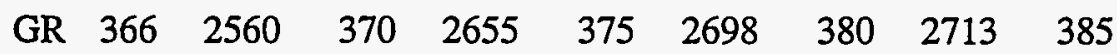

2750

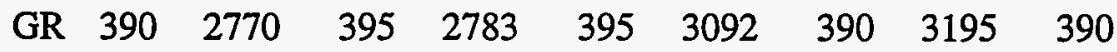

3320

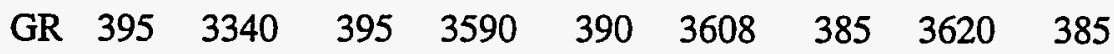

3744

$\begin{array}{llllllllll}\text { GR } & 390 & 3756 & 395 & 3768 & 400 & 3780 & 400 & 4450 & 380\end{array}$

4465

$\begin{array}{lllllllll}\text { GR } & 380 & 4512 & 400 & 4595 & 425 & 4725 & 440 & 4775\end{array}$

$\begin{array}{llllllll}\mathrm{X} 1 & 23.2 & 27 & 0 & 1768 & 5280 & 5280 & 5280\end{array}$

$\begin{array}{llllllllll}\text { GR } & 440 & .0 & 425 & 15 & 415 & 20 & 410 & 50 & 400\end{array}$

60

$\begin{array}{llllllllll}\text { GR } & 380 & 85 & 375 & .200 & 370 & 240 & 365.3 & 300 & 368.5\end{array}$

370

$\begin{array}{llllllllll}\text { GR } & 370 & 482 & 370 & 502 & 365 & 585 & 365 & 675 & 370\end{array}$

700

$\begin{array}{llllllllll}\text { GR } & 375 & 763 & 380 & 868 & 390 & 970 & 400 & 1050 & 405\end{array}$

1152

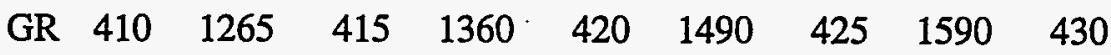

1668

$\begin{array}{lllll}\text { GR } & 435 & 1710 & 440 & 1768\end{array}$

$\begin{array}{llllllll}\mathrm{X} 1 & 24.3 & 21 & 0 & 2380 & 5870 & 5870 & 5870\end{array}$

$\begin{array}{llllllllll}\text { GR } & 440 & 0 & 435 & 142 & 430 & 275 & 425 & 425 & 420\end{array}$

505

$\begin{array}{llllllllll}\text { GR } & 420 & 670 & 415 & 700 & 410 & 725 & 385 & 828 & 380\end{array}$

900

$\begin{array}{llllllllll}\text { GR } & 377 & 1483 & 376 & 1590 & 376 & 1810 & 377 & 1920 & 377.7\end{array}$

2030

$\begin{array}{llllllllll}\text { GR } & 380 & 2150 & 380 & 2168 & 375 & 2243 & 375 & 2310 & 380\end{array}$ 2370

GR $440 \quad 2380$

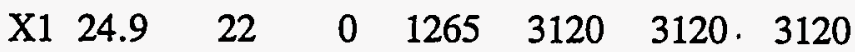




\begin{tabular}{|c|c|c|c|c|c|c|c|c|}
\hline $\begin{array}{ll}\text { GR } & 440 \\
245 & \end{array}$ & 0 & 430 & 80 & 425 & 130 & 410 & 194 & 405 \\
\hline $\begin{array}{ll}\text { GR } & 400 \\
850\end{array}$ & 302 & 385 & 498 & 381 & 608 & 375 & 700 & 370 \\
\hline $\begin{array}{l}\text { GR } 365 \\
1150\end{array}$ & 948 & 360 & 1020 & 355 & 1088 & 350 & 1098 & 350 \\
\hline $\begin{array}{l}\text { GR } 355 \\
1240\end{array}$ & 1165 & 370 & 1190 & 375 & 1210 & 380 & 1230 & 400 \\
\hline GR $\quad 425$ & 1253 & 440 & 1265 & & & & & \\
\hline 26 & 29 & 02 & 2460 & 5810 & 5810 & 5810 & & \\
\hline $\begin{array}{ll}\text { GR } & 440 \\
70 & \end{array}$ & 0 & 430 & 12 & 425 & 18 & 420 & 42 & 410 \\
\hline $\begin{array}{ll}\text { GR } & 400 \\
448 & \end{array}$ & 82 & 385 & 118 & 380 & 208 & 375 & 308 & 370 \\
\hline 369 & 475 & 370 & 530 & 375 & 630 & 380 & 820 & 385 \\
\hline $\begin{array}{l}\text { GR } 390 \\
1675\end{array}$ & 1038 & 390 & 1215 & 395 & 1282 & 400 & 1663 & 405 \\
\hline $\begin{array}{l}\text { GR } 410 \\
2210\end{array}$ & 1686 & 415 & 1730 & 415 & 1890 & 415 & 2056 & 420 \\
\hline GR $\quad 425$ & 2331 & 430 & 2380 & 435 & 2418 & 440 & 2460 & \\
\hline$\times 126.9$ & 33 & 0 & 2480 & 4810 & 4810 & 4810 & & \\
\hline $\begin{array}{ll}\text { GR } & 440 \\
440 & \end{array}$ & 0 & 425 & 40 & 420 & 58 & 415 & 115 & 410 \\
\hline $\begin{array}{ll}\text { GR } & 405 \\
550 & \end{array}$ & 457 & 405 & 495 & 410 & 500 & 420 & 530 & 420 \\
\hline $\begin{array}{l}\text { JR } 415 \\
135\end{array}$ & 580 & 415 & 850 & 415 & 1030 & 410 & 1095 & 405 \\
\hline $\begin{array}{l}\text { GR } 400 \\
1482\end{array}$ & 1170 & 395 & 1210 & 390 & 1258 & 385 & 1352 & 380 \\
\hline $\begin{array}{l}\text { GR } 375 \\
2160\end{array}$ & 1610 & 374 & 1830 & 375 & 1940 & 380 & 2092 & 385 \\
\hline $\begin{array}{l}\text { GR } 395 \\
2382\end{array}$ & 2248 & 400 & 2325 & 405 & 2348 & 410 & 2358 & 415 \\
\hline GR $\quad 420$ & 2410 & 425 & 2425 & 440 & 2480 & & & \\
\hline $\mathrm{X} 1 \quad 28$ & 24 & 03 & 31005 & 5830 & 5830 & 5830 & & \\
\hline $\begin{array}{l}\text { GR } 440 \\
1018\end{array}$ & 0 & 425 & 1 & 420 & 110 & 420 & 148 & 420 \\
\hline $\begin{array}{l}\text { GR } 420 \\
1715\end{array}$ & 1058 & 415 & 1380 & 420 & 1525 & 420 & 1700 & 415 \\
\hline $\begin{array}{l}\text { GR } 410 \\
2145\end{array}$ & 1718 & 405 & 1745 & 400 & 1763 & 395 & 1850 & 386 \\
\hline $\begin{array}{l}\text { GR } 385 \\
3000\end{array}$ & 2265 & 381 & 2475 & 385 & 2880 & 386 & .2960 & 390 \\
\hline $\begin{array}{lr}\text { GR } & 395 \\
\times 1 & 285\end{array}$ & 3022 & 400 & 3030 & 425 & 3075 & 440 & 3100 & \\
\hline $\begin{array}{lr}\text { X1 } & 28.5 \\
\text { GR } & 440\end{array}$ & $\begin{array}{r}34 \\
0\end{array}$ & $\begin{array}{c}0 \\
425\end{array}$ & $\begin{array}{r}2560 \\
25\end{array}$ & $\begin{array}{c}2640 \\
420\end{array}$ & $\begin{array}{c}2640 \\
35\end{array}$ & $\begin{array}{l}2640 \\
415\end{array}$ & 65 & 10 \\
\hline
\end{tabular}


90

$\begin{array}{llllllllll}\text { GR } & 410 & 115 & 420 & 120 & 420 & 160 & 415 & 170 & 415\end{array}$

230

$\begin{array}{llllllllll}\text { GR } & 415 & 620 & 410 & 752 & 405 & 820 & 400 & 842 & 395\end{array}$

$\begin{array}{llllllllll}875 & & & & & & & & & \\ \text { GR } & 390 & 970 & 389.9 & 972 & 389 & 1020 & 388 & 1070 & 388\end{array}$

1120

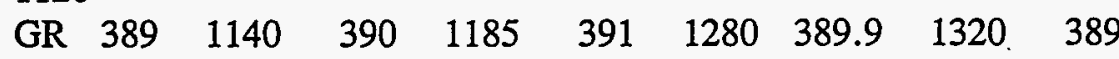

1380

$\begin{array}{llllllllll}\text { GR } & 388 & 1420 & 387 & 1550 & 387 & 1640 & 388 & 1700 & 389\end{array}$

1740

$\begin{array}{lllllllll}\text { GR } & 390 & 1760 & 395 & 2350 & 400 & 2442 & 440 & 2560\end{array}$

$\begin{array}{llllllll}\mathrm{X} 1 & 29.7 & 29 & 0 & 2870 & 6350 & 6350 & 6350\end{array}$

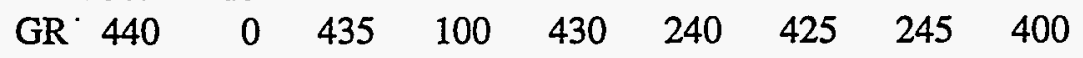

290

$\begin{array}{llllllllll}\text { GR } & 400 & 479 & 405 & 492 & 410 & 498 & 415 & 512 & 420\end{array}$

610

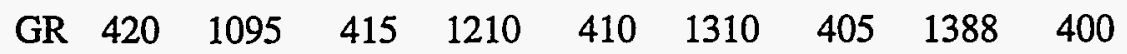

1495

$\begin{array}{llllllllll}\text { GR } & 395 & 1610 & 393 & 1710 & 390 & 1932 & 385 & 2202 & 390\end{array}$

2385

$\begin{array}{llllllllll}\text { GR } & 393 & 2510 & 395 & 2568 & 400 & 2618 & 405 & 2670 & 410\end{array}$

2700

$\begin{array}{lllllllll}\text { GR } & 415 & 2760 & 420 & 2795 & 425 & 2828 & 440 & 2870\end{array}$

$\begin{array}{llllllll}\mathrm{X} 1 & 30.8 & 20 & 0 & 2080 & 5680 & 5680 & 5680\end{array}$

$\begin{array}{llllllllll}\text { GR } & 440 & 0 & 425 & 29 & 415 & 50 & 410 & 72 & 405\end{array}$

80

$\begin{array}{llllllllll}\text { GR } & 400 & 103 & 395 & 302 & 394.2 & 350 & 390 & 530 & 388.2\end{array}$

590

$\begin{array}{llllllllll}\text { GR } & 386 & 665 & 386 & 753 & 390 & 858 & 395 & 1080 & 400\end{array}$

1340

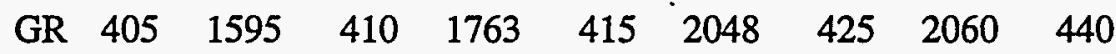

2080

$\begin{array}{lllllllll}\mathrm{X} 131.50 & 32.0 & .0 & 2400.0 & 3720.0 & 3720.0 & 3720.0 & .000 & .000\end{array}$ .000

$\begin{array}{lllllllll}\text { GR } 440.0 & .0 & 435.0 & 8.0 & 430.0 & 42.0 & 425.0 & 48.0 & 400.0\end{array}$ .85 .0

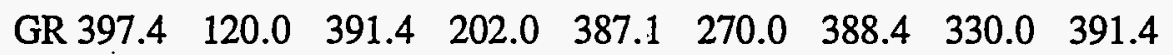
390.0

$\begin{array}{lllllllll}\text { GR } 397.4 & 440.0 & 400.0 & 510.0 & 405.0 & 892.0 & 410.0 & 1030.0 & 415.0\end{array}$ 1130.0

$\begin{array}{lllllllll}\text { GR } 415.0 & 1390.0 & 410.0 & 1490.0 & 405.0 & 1615.0 & 400.0 & 1735.0 & 397.4\end{array}$ 1860.0

$\begin{array}{lllllllll}\text { GR } 395.6 & 1960.0 & 397.4 & 2005.0 & 400.0 & 2082.0 & 405.0 & 2155.0 & 410.0\end{array}$ 2205.0

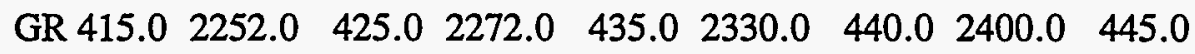
2670.0 
GR $450.0 \quad 2785.0 \quad 475.0 \quad 2875.0$

$\begin{array}{lllllllll}\mathrm{X} 132.00 & 46.0 & .0 & 2297.0 & 2700.0 & 2700.0 & 2700.0 & .000 & .000\end{array}$ .000

$\begin{array}{lllllllll}\text { GR } 440.0 & .0 & 435.0 & 6.0 & 430.0 & 12.0 & 430.0 & 22.0 & 430.0\end{array}$

45.0

$\begin{array}{lllllllll}\text { GR } 425.0 & 65.0 & 420.0 & 75.0 & 415.0 & 100.0 & 410.0 & 112.0 & 405.0\end{array}$ 122.0

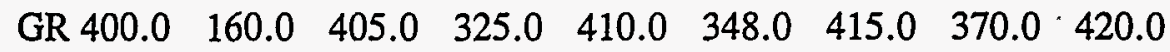
500.0

GR $415.0 \quad 550.0 \quad 415.0 \quad 580.0 \quad 425.0 \quad 610.0 \quad 425.0 \quad 740.0 \quad 420.0$ 775.0

$\begin{array}{lllllllll}\text { GR } 415.0 & 825.0 & 410.0 & 1070.0 & 405.0 & 1125.0 & 400.0 & 1200.0 & 398.7\end{array}$ 1240.0

$\begin{array}{lllllllll}\text { GR } 395.0 & 1325.0 & 390.0 & 1485.0 & 387.0 & 1580.0 & 386.6 & 1637.0 & 387.0\end{array}$ 1690.0

$\begin{array}{lllllllll}\text { GR } 390.0 & 1790.0 & 395.0 & 1850.0 & 400.0 & 2048.0 & 405.0 & 2115.0 & 410.0\end{array}$ 2142.0

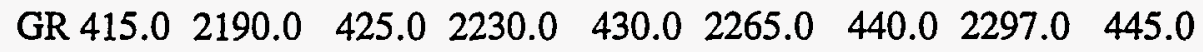
2310.0

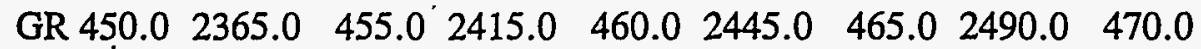
2535.0

GR 475.02645 .0 .

$\begin{array}{llllllll}\mathrm{X} 133.00 & 28.0 & 825.0 & 2700.0 & 5230.0 & 5230.0 & 5230.0 & .000\end{array} \cdot .000$ .000

$\begin{array}{lllllllll}\text { GR } 475.0 & .0 & 470.0 & 40.0 & 465.0 & 80.0 & 460.0 & 200.0 & 460.0\end{array}$

380.0

$\begin{array}{lllllllll}\text { GR } 455.0 & 425.0 & 450.0 & 450.0 & 445.0 & 635.0 & 440.0 & 825.0 & 425.0\end{array}$

863.0

$\begin{array}{lllllllll}\text { GR } 420.0 & 882.0 & 415.0 & 912.0 & 415.0 & 1059.0 & 415.0 & 1351.0 & 410.0\end{array}$

1387.0

$\begin{array}{lllllllll}\text { GR } 405.0 & 1445.0 & 400.0 & 2157.0 & 395.0 & 2255.0 & 394.2 & 2277.0 & 393.0\end{array}$

2315.0

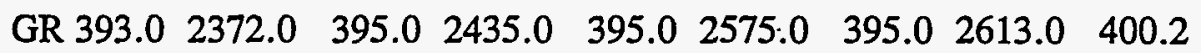
2635.0

$\begin{array}{llllll}\text { GR } 405.0 \quad 2655: 0 & 425.0 & 2685.0 & 440.0 & 2700.0\end{array}$

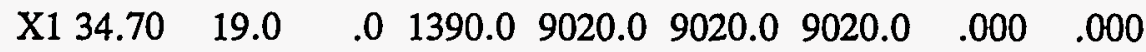
.000

$\begin{array}{lllllllll}\text { GR } 440.0 & .0 & 435.0 & 28.0 & 430.0 & 53.0 & 425.0 & 86.0 & 420.0\end{array}$ 105.0

$\begin{array}{lllllllll}\text { GR } 415.0 & 132.0 & 410.0 & 205.0 & 405.0 & 341.0 & 400.0 & 625.0 & 395.0\end{array}$ 762.0

$\begin{array}{lllllllll}\text { GR } 394.0 & 798.0 & 395.0 & 810.0 & 400.0 & 907.0 & 405.0 & 962.0 & 410.0\end{array}$ 1270.0

$\begin{array}{llllllll}\text { GR } 415.0 & 1348.0 & 420.0 & 1360.0 & 425.0 & 1370.0 & 440.0 & 1390.0\end{array}$

$\begin{array}{llllllllll}\mathrm{X} 135.40 & 16.0 & .0 & 1485.0 & 3680.0 & 3680.0 & 3680.0 & .000 & .000\end{array}$ .000

$\begin{array}{lllllllll}\text { GR } 440.0 & .0 & 430.0 & 29.0 & 425.0 & 75.0 & 420.0 & 152.0 & 415.0\end{array}$ 
225.0

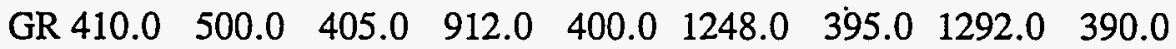
1332.0

GR $390.0 \quad 1409.0 \quad 395.0 \quad 1420.0 \quad 400.0 \quad 1432.0 \quad 410.0 \quad 1448.0 \quad 425.0$ 1472.0

GR 440.01485 .0

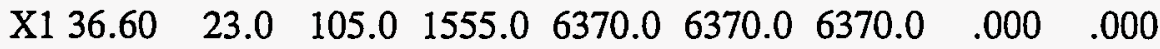
.000

$\begin{array}{lllllllll}\text { GR } 475.0 & .0 & 460.0 & 15.0 & 460.0 & 75.0 & 450.0 & 85.0 & 440.0\end{array}$

105.0

GR 430.0 $\quad 117.0 \quad 425.0 \quad 137.0 \quad 420.0 \quad 162.0 \quad 417.5 \quad 204.0 \quad 415.0$

223.0

$\begin{array}{lllllllll}\text { GR } 410.0 & 495.0 & 408.0 & 530.0 & 410.0 & 803.0 & 411.5 & 975.0 & 415.0\end{array}$

1385.0

$\begin{array}{lllllllll}\text { GR } 420.0 & 1430.0 & 425.0 & 1487.0 & 430.0 & 1505.0 & 435.0 & 1535.0 & 440.0\end{array}$ 1555.0

GR $445.0 \quad 1635.0 \quad 450.0 \quad 1700.0 \quad 475.0 \quad 1790.0$

$\begin{array}{lllllllll}\mathrm{X} 137.40 & 27.0 & 140.0 & 1385.0 & 4120.0 & 4120.0 & 4120.0 & .000 & .000\end{array}$ .000

$\begin{array}{lllllllll}\text { GR } 475.0 & .0 & 470.0 & 40.0 & 455.0 & 70.0 & 450.0 & 107.0 & 440.0\end{array}$

140.0

$\begin{array}{lllllllll}\text { GR } 435.0 & 148.0 & 430.0 & 186.0 & 425.0 & 227.0 & 420.0 & 252.0 & 418.3\end{array}$

302.0

$\begin{array}{lllllllll}\text { GR } 415.0 & 420.0 & 410.0 & 567.0 & 405.0 & 732.0 & 402.0 & 893.0 & 402.0\end{array}$

1002.0

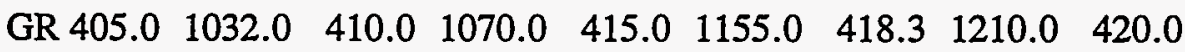

1235.0

$\begin{array}{lllllllll}\text { GR } 425.0 & 1265.0 & 430.0 & 1340.0 & 435.0 & 1370.0 & 440.0 & 1385.0 & 445.0\end{array}$

1540.0

GR $450.0 \quad 1655.0 \quad 475.0 \quad 1690.0$

$\begin{array}{lllllllll}\mathrm{X} 138.50 & 26.0 & 70.0 & 1065.0 & 5820.0 & 5820.0 & 5820.0 & .000 & .000\end{array}$ .000

$\begin{array}{lllllllll}\text { GR } 475.0 \quad & .0 & 455.0 & 15.0 & 450.0 & 52.0 & 440.0 & 70.0 & 425.0\end{array}$

100.0

$\begin{array}{lllllllll}\text { GR } 420.0 & 125.0 & 415.0 & 150.0 & 410.0 & 160.0 & 405.0 & 190.0 & 400.0\end{array}$

220.0

$\begin{array}{lllllllll}\text { GR } 399.0 & 260.0 & 400.0 & 310.0 & 405.0 & 602.0 & 410.0 & 710.0 & 415.0\end{array}$

870.0

$\begin{array}{lllllllll}\text { GR } 420.0 & 935.0 & 425.0 & 1000.0 & 430.0 & 1035.0 & 440.0 & 1065.0 & 445.0\end{array}$

1075.0

GR $450.0 \quad 1170.0 \quad 455.0 \quad 1225.0 \quad 460.0 \quad 1300.0 \quad 465.0 \quad 1385.0 \quad 470.0$

1430.0

GR 475.01495 .0

$\begin{array}{lllllllll}\mathrm{X} 139.60 & 25.0 & 435.0 & 1710.0 & 5470.0 & 5470.0 & 5470.0 & .000 & .000\end{array}$

.000

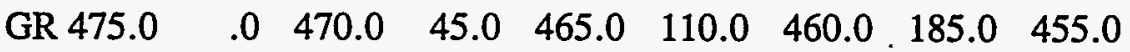

225.0 
$\begin{array}{lllllllll}\text { GR } 455.0 & 265.0 & 450.0 & 290.0 & 445.0 & 325.0 & 440.0 & 435.0 & 435.0\end{array}$ 473.0

$\begin{array}{lllllllll}\text { GR } 430.0 & 483.0 & 425.0 & 495.0 & 422.1 & 537.0 & 420.0 & 685.0 & 418.0\end{array}$ 1035.0

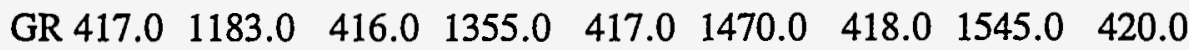
1607.0

$\begin{array}{lllllllll}\text { GR } 422.1 & 1645.0 & 425.0 & 1678.0 & 440.0 & 1710.0 & 450.0 & 1730.0 & 475.0\end{array}$ 1775.0

$\begin{array}{lllllllll}\mathrm{X} 140.70 & 24.0 & 75.0 & 1285.0 & 5820.0 & 5820.0 & 5820.0 & .000 & .000\end{array}$ .000

$\begin{array}{lllllllll}\text { GR } 475.0 & .0 & 465.0 & 11.0 & 460.0 & 28.0 & 450.0 & 53.0 & 440.0\end{array}$

75.0

$\begin{array}{lllllllll}\text { GR } 430.0 & 93.0 & 425.0 & 114.0 & 422.8 & 140.0 & 420.0 & 255.0 & 416.8\end{array}$

503.0

$\begin{array}{lllllllll}\text { GR } 415.0 & 603.0 & 414.0 & 623.0 & 415.0 & 785.0 & 416.8 & 835.0 & 420.0\end{array}$

965.0

$\begin{array}{lllllllll}\text { GR } 422.8 & 1156.0 & 425.0 & 1185.0 & 430.0 & 1213.0 & 435.0 & 1245.0 & 440.0\end{array}$

1285.0

$\begin{array}{llllllll}\text { GR } 445.0 & 1300.0 & 450.0 & 1310.0 & 460.0 & 1335.0 & 475.0 & 1355.0\end{array}$

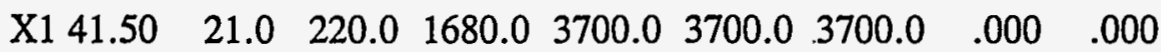
.000

$\begin{array}{lllllllll}\text { GR } 475.0 & .0 & 470.0 & 95.0 & 465.0 & 135.0 & 450.0 & 155.0 & 445.0\end{array}$

170.0

$\begin{array}{lllllllll}\text { GR } 440.0 & 220.0 & 435.0 & 240.0 & 430.0 & 250.0 & 425.8 & 420.0 & 425.0\end{array}$

500.0

$\begin{array}{lllllllll}\text { GR } 420.0 & 770.0 & 420.0 & 820.0 & 425.8 & 972.0 & 425.8 & 1395.0 & 425.0\end{array}$

1470.0

$\begin{array}{lllllllll}\text { GR } 425.8 & 1530.0 & 430.0 & 1645.0 & 435.0 & 1660.0 & 440.0 & 1680.0 & 455.0\end{array}$

1715.0

GR 475.01805 .0

$\begin{array}{lrrrrrrrrr}\text { X1 } & 41.59 & 31 & 0 & 2140 & 527 & 527 & 527 & & \\ \text { GR } & 500 & 0 & 475 & 130 & 470 & 170 & 465 & 210 & 450\end{array}$

235

$\begin{array}{llllllllll}\text { GR } & 445 & 265 & 440 & 310 & 435 & 335 & 430 & 350 & 426\end{array}$

$\begin{array}{llllllllll}505 & & & & & & & & \\ \text { GR } & 420 & 700 & 418.7 & 810 & 420 & 890 & 426 & 1050 & 426\end{array}$

1262

$\begin{array}{lllllllll}\text { GR } 423.8 & 1350 & 426 & 1600 & 430 & 1680 & 435 & 1700 & 440\end{array}$

1708

$\begin{array}{llllllllll}\text { GR } & 465 & 1800 & 470 & 1830 & 475 & 1898 & 480 & 1940 & 485\end{array}$

1965

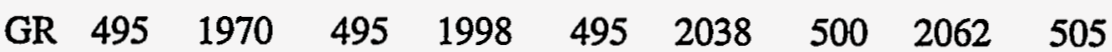

2080

GR $510 \quad 2140$

* LOWER MONUMENTAL DAM. RM 41.6

$\begin{array}{llllllll}\mathrm{X} 1 & 41.6 & 31 & 0 & 2140 & 1 & 1 & 1\end{array}$

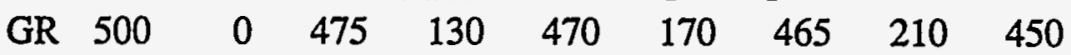




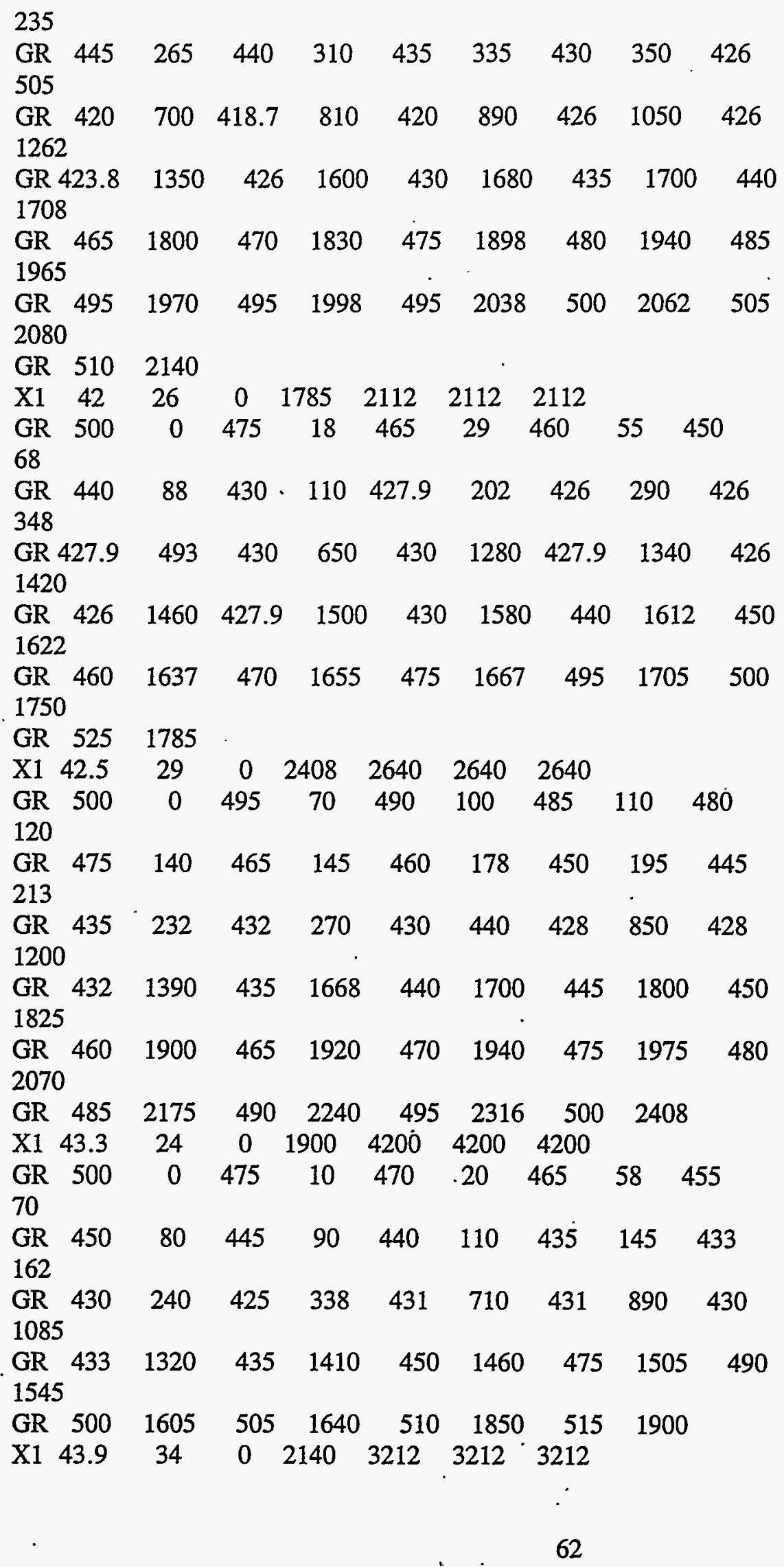




\begin{tabular}{|c|c|c|c|c|c|c|c|c|}
\hline $\begin{array}{cc}\text { GR } & 500 \\
142 & \end{array}$ & 0 & 495 & 28 & 490 & 48 & 485 & 90 & 480 \\
\hline $\begin{array}{ll}\text { GR } & 475 \\
360\end{array}$ & 164 & 470 & 208 & 465 & 248 & 465 & 320 & 465 \\
\hline $\begin{array}{ll}\text { GR } & 460 \\
760\end{array}$ & 400 & 455 & 560 & 450 & 610 & 445 & 690 & 440 \\
\hline $\begin{array}{l}\text { GR } 435 \\
1590\end{array}$ & 955 & 430 & 1010 & 425 & 1100 & 428.2 & 1500 & 430 \\
\hline $\begin{array}{l}\text { GR } 434.2 \\
1982\end{array}$ & 1735 & 434.2 & 1930 & 435 & 1950 & 440 & 1970 & 445 \\
\hline $\begin{array}{l}\text { GR } 450 \\
2050\end{array}$ & 2000 & 460 & 2008 & 470 & 2028 & 475 & 2032 & 485 \\
\hline JR 500 & 2068 & 510 & 2083 & 520 & 2110 & 525 & 2140 & \\
\hline$X 144.65$ & 21 & 0 & 1565 & 3940 & 3940 & 3940 & & \\
\hline $\begin{array}{ll}\text { GR } & 500 \\
55 & \end{array}$ & 0 & 495 & 20 & 490 & 45 & 485 & 62 & 480 \\
\hline 475 & 75 & 470 & 105 & 450 & 200 & 445 & 220 & 440 \\
\hline $\begin{array}{l}\text { GR } 436 \\
1140\end{array}$ & 830 & 435 & 960 & 430 & 1015 & 425 & 1070 & 422 \\
\hline $\begin{array}{l}\text { GR } 425 \\
1550\end{array}$ & 1200 & 430 & 1308 & 436 & 1510 & 440 & 1538 & 500 \\
\hline$R^{\prime} 525$ & 1565 & & & & & & & \\
\hline 45.2 & 31 & 0 & 2100 & 2930 & 2930 & 2930 & & \\
\hline $\begin{array}{ll}\text { GR } & 560 \\
420 & \end{array}$ & 0 & 540 & 200 & 520 & 320 & 500 & 400 & 495 \\
\hline $\begin{array}{ll}\text { GR } & 475 \\
580 & \end{array}$ & 445 & 470 & 470 & 450 & 510 & 445 & 540 & 440 \\
\hline $\begin{array}{l}\text { GR } 438.8 \\
980\end{array}$ & 620 & 435 & 700 & 430 & 790 & 427 & 875 & 425 \\
\hline $\begin{array}{l}\text { GR } 425 \\
1835\end{array}$ & 1035 & 430 & 1060 & 435 & 1130 & 438.8 & 1145 & 440 \\
\hline $\begin{array}{l}\text { GR } 445 \\
1948\end{array}$ & 1852 & 450 & 1870 & 455 & 1915 & 460 & 1930 & 465 \\
\hline $\begin{array}{l}\text { GR } 470 \\
2060\end{array}$ & 1960 & 475 & 1975 & 500 & 2000 & 505 & 2030 & 525 \\
\hline 555 & 2100 & & & & & & & \\
\hline 46 & 23 & 0 & 1615 & 4190 & 4190 & 4190 & & \\
\hline $\begin{array}{ll}\text { GR } & 500 \\
110 & \end{array}$ & 0 & 495 & 20 & 475 & 55 & 470 & 80 & 50 \\
\hline GR 445 & 120. & 440 & 200 & 440 & 240 & 435 & 690 & 430 \\
\hline $\begin{array}{l}\text { GR } 428 \\
1280\end{array}$ & 912 & 430 & 928 & 435 & 1080 & 440 & 1235 & 445 \\
\hline $\begin{array}{l}\text { GR } 450 \\
1405\end{array}$ & 1310 & 455 & 1340 & 460 & 1355 & 470 & 1380 & 475 \\
\hline GR $\quad 500$ & 1545 & 505 & .1580 & 525 & 1615 & & & \\
\hline
\end{tabular}




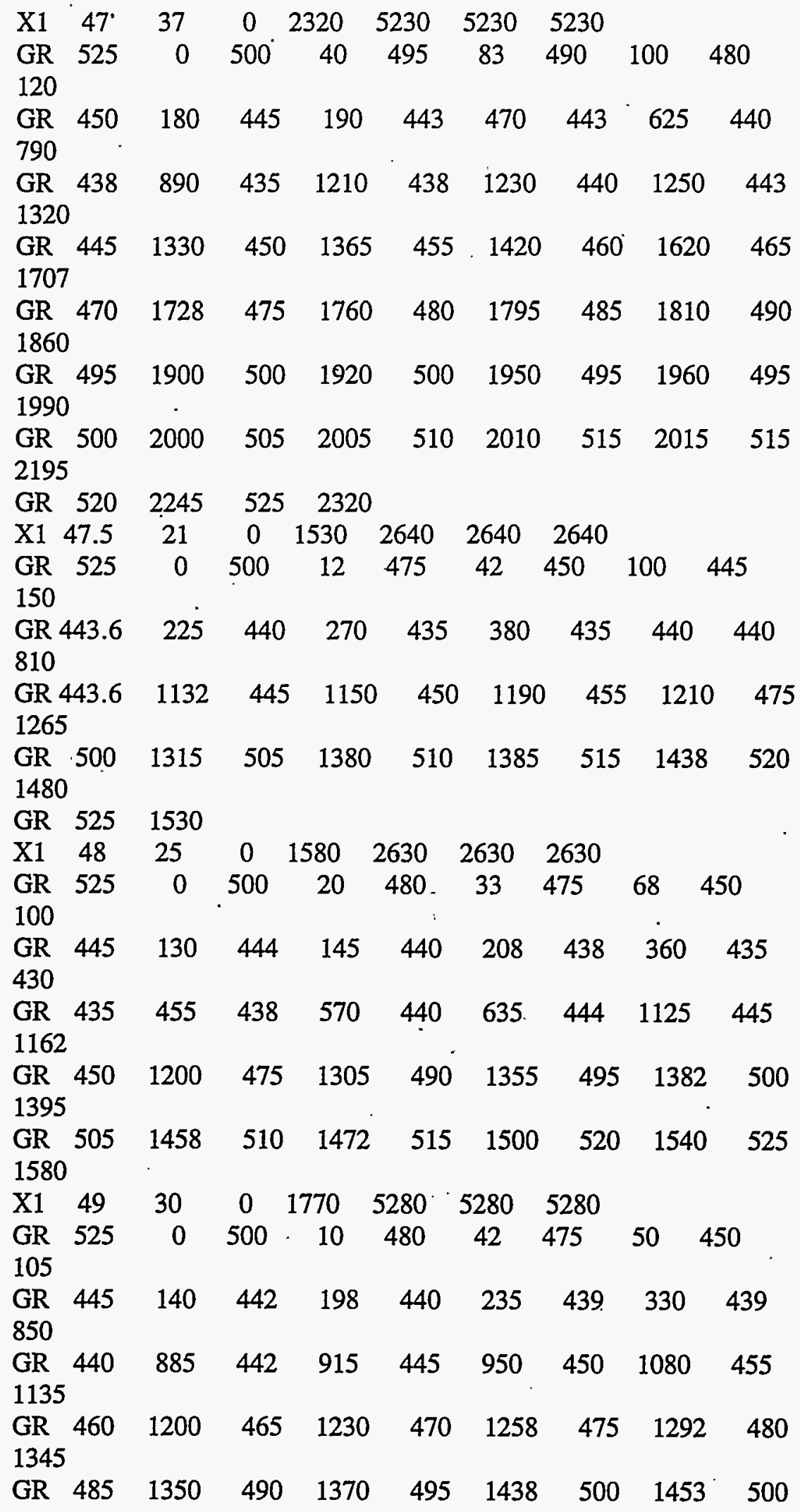


1530

$\begin{array}{llllllllll}\text { GR } & 505 & 1560 & 510 & 1600 & 515 & 1625 & 520 & 1705 & \end{array}$ 1770

$\begin{array}{llllllll}\mathrm{X} 1 & 50 & 32 & 0 & 2353 & 5280 & 5280 & 5280\end{array}$

$\begin{array}{llllllllll}\text { GR } & 525 & 0 & 520 & 62 & .515 & 115 & 510 & 158 & 505\end{array}$

198

$\begin{array}{llllllllll}\text { GR } & 500 & 235 & 495 & 345 & 490 & 410 & 485 & 640 & 480\end{array}$

685

$\begin{array}{llllllllll}\text { GR } & 475 & 715 & 470 & 760 & 465 & 805 & 460 & 830 & 455\end{array}$

840

$\begin{array}{llllllllll}\text { GR } & 450 & 1045 & 445 & 1275 & 442 & 1310 & 444 & 1680 & 445\end{array}$

1715

$\begin{array}{llllllllll}\text { GR } & 450 & 1950 & 455 & 2034 & 475 & 2072 & 485 & 2097 & 495\end{array}$

2118

$\begin{array}{llllllllll}\text { GR } & 500 & 2150 & 505 & 2200 & 505 & 2258 & 510 & 2290 & 515\end{array}$ 2302

$\begin{array}{lllll}\text { GR } & 520 & 2325 & 525 & 2353\end{array}$

$\begin{array}{llllllll}\mathrm{X} 1 & 51 & 27 & 0 & 1490 & 5280 & 5280 & 5280\end{array}$

$\begin{array}{llllllllll}\text { GR } & 525 & 0 & 520 & 98 & 515 & 102 & 510 & 111 & 505\end{array}$

174

$\begin{array}{llllllllll}\text { GR } & 500 & 190 & 495 & 210 & 490 & 240 & 475 & 270 & 470\end{array}$

280

$\begin{array}{llllllllll}\text { GR } & 465 & 315 & 460 & 355 & 455 & 418 & 450 & 695 & 445\end{array}$

$\begin{array}{llllllllll}760 & & & & & & & & & \\ \text { GR } & 442 & 775 & 440 & 790 & 429 & 818 & 442 & 830 & 445\end{array}$

$\begin{array}{lllllllll}860 & & & & & & & & \end{array}$

$\begin{array}{llllllllll}\text { GR } & 450 & 905 & 455 & 1300 & 460 & 1335 & 475 & 1390 & 500\end{array}$

1440

$\begin{array}{lllll}\text { GR } & 505 & 1480 & 525 & 1490\end{array}$

$\begin{array}{llllllll}\mathrm{X} 1 & 52 & 30 & 0 & 1400 & 5280 & 5280 & 5280\end{array}$

$\begin{array}{llllllllll}\text { GR } & 530 & 0 & 525 & 41 & 520 & 78 & 515 & 110 & 510\end{array}$

135

$\begin{array}{llllllllll}\text { GR } & 505 & 170 & 500 & 190 & 475 & 248 & 470 & 268 & 465\end{array}$

307

$\begin{array}{llllllllll}\text { GR } & 460 & 372 & 455 & 425 & 450 & 545 & 445 & 680 & 440\end{array}$

720

$\begin{array}{llllllllll}\text { GR } & 435 & 760 & 433 & 820 & 435 & 870 & 440 & 950 & 445\end{array}$

1025

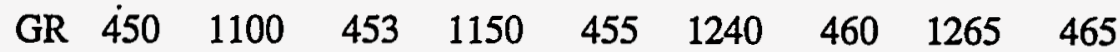

1290

$\begin{array}{llllllllll}\text { GR } & 470 & 1320 & 475 & 1330 & 500 & 1358 & 505 & 1385 & 525\end{array}$ 1400

$\begin{array}{llllllll}\mathrm{X} 1 & 53 & 29 & 0 & 1375 & 5280 & 5280 & 5280\end{array}$

$\begin{array}{llllllllll}\text { GR } & 535 & 0 & 530 & 60 & 525 & 92 & 520 & 130 & 515\end{array}$

165

$\begin{array}{llllllllll}\text { GR } & 510 & 205 & 505 & 225 & 500 & 275 & 475 & 338 & 470\end{array}$ 


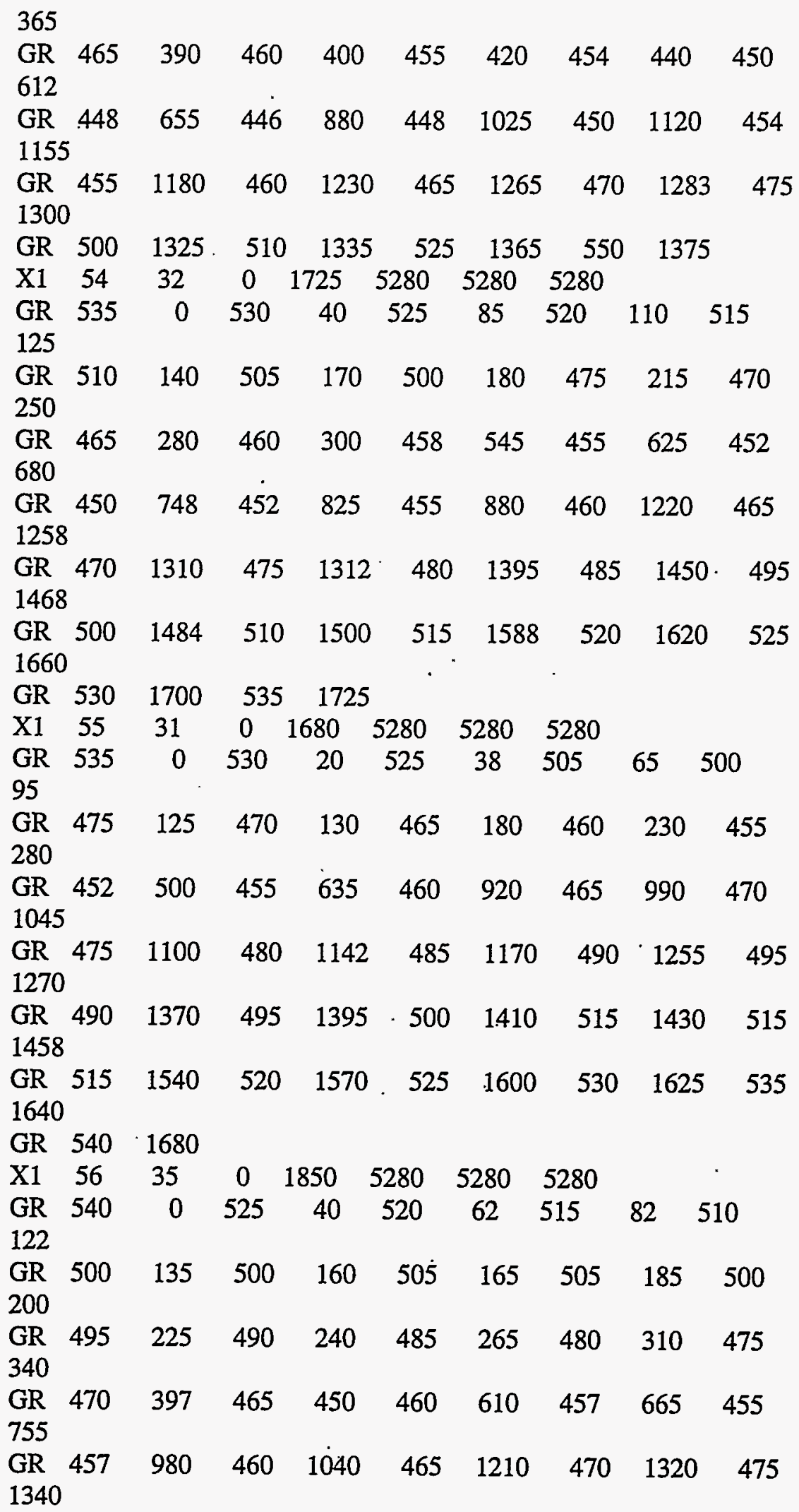


$\begin{array}{llllllllll}\text { GR } & 480 & 1350 & 500 & 1405 & 505 & 1440 & 510 & 1490 & 515\end{array}$ 1530

$\begin{array}{llllllllll}\text { GR } & 520 & 1555 & 525 & 1645 & 530 & 1710 & .535 & 1782 & 540\end{array}$ 1850

$\begin{array}{llllllll}\mathrm{X} 1 & 57 & 28 & 0 & 1150 & 5280 & 5280 & 5280\end{array}$

$\begin{array}{llllllllll}\mathrm{GR} & 540 & 0 & 535 & 28 & 530 & 50 & 525 & 70 & 520\end{array}$

$\begin{array}{lccccccccc}88 & \cdot & & & & & & & \\ \text { GR } & 500 & 145 & 475 & 200 & 470 & 208 & 466 & 280 & 460\end{array}$

$\begin{array}{llllllllll}340 & & & & & & & & & \\ \text { GR } & 455 & 400 & 450 & 450 & 445 & 550 & 442 & 555 & 445\end{array}$

580

$\begin{array}{llllllllll}\text { GR } & 450 & 605 & 455 & 700 & 460 & 790 & 465 & 870 & 470\end{array}$

955

$\begin{array}{llllllllll}\text { GR } & 475 & 975 & 480 & 995 & 485 & 1015 & 490 & 1040 & 500\end{array}$ 1050

$\begin{array}{lllllll}\text { GR } & 520 & 1075 & 525 & 1113 & 550 & 1150\end{array}$

$\begin{array}{llllllll}\mathrm{X} 1 & 58 & 33 & 0 & 1600 & 5280 & 5280 & 5280\end{array}$

$\begin{array}{llllllllll}\text { GR } & 550 & 0 & 525 & 40 & 510 & 60 & 505 & 82 & 500\end{array}$

92

$\begin{array}{llllllllll}\text { GR } & 495 & 108 & 485 & 125 & 480 & 145 & 475 & 165 & 470\end{array}$

230

$\begin{array}{llllllllll}\text { GR } & 469 & 570 & 467 & 590 & 465 & 642 & 467 & 880 & 469\end{array}$

950

$\begin{array}{llllllllll}\text { GR } & 470 & 1090 & 475 & 1160 & 480 & 1200 & 485 & 1230 & 490\end{array}$

1245

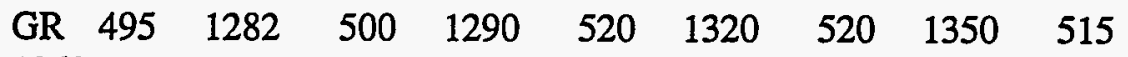

1360

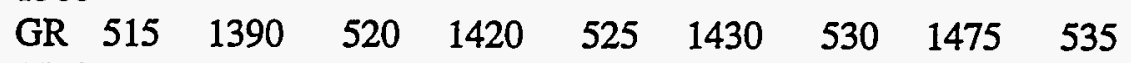

1510

$\begin{array}{lllllll}\text { GR } & 540 & 1562 & 545 & 1580 & 550 & 1600\end{array}$

$\begin{array}{llllllll}\mathrm{X} 1 & 59 & 36 & 0 & 1620 & 5280 & 5280 & 5280\end{array}$

$\begin{array}{llllllllll}\text { GR } & 550 & 0 & 525 & 24 & 520 & 108 & 515 & 130 & 510\end{array}$

160

$\begin{array}{llllllllll}\text { GR } & 500 & 180 & 495 & 183 & 490 & 208 & 485 & 245 & 480\end{array}$

275

$\begin{array}{llllllllll}\text { GR } & 475 & 300 & 472 & 450 & 470 & 725 & 465 & 840 & 460\end{array}$

885

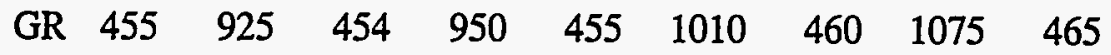

1120

$\begin{array}{llllllllll}\text { GR } & 470 & 1190 & 475 & 1215 & 480 & 1240 & 485 & 1275 & 490\end{array}$

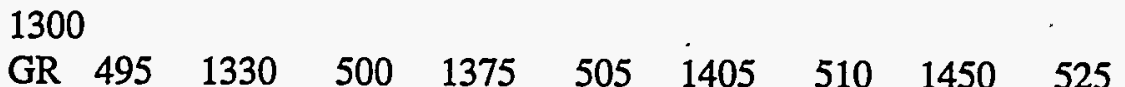

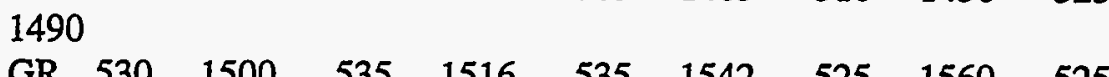

$\begin{array}{llllllllll}\text { GR } & 530 & 1500 & 535 & 1516 & 535 & 1542 & 525 & 1560 & 525\end{array}$

1580

GR $550 \quad 1620$

$\begin{array}{llllllll}\mathrm{X} 1 & 60 & 30 & 0 & 1182 & 5280 & 5280 & 5280\end{array}$ 
$\begin{array}{llllllllll}\text { GR } & 565 & 0 & 550 & 13 & 525 & 38 & 515 & 60 & 500\end{array}$

87

$\begin{array}{llllllllll}\text { GR } & 495 & 110 & 490 & 195 & 485 & 260 & 485 & 325 & 485\end{array}$

508

$\begin{array}{llllllllll}\text { GR } & 480 & 550 & 475 & 570 & 470 & 580 & 465 & 590 & 450\end{array}$

$\begin{array}{llllllllll}595 & & & & & & & & & \\ \text { GR } & 440 & 615 & 434 & 660 & 440 & 690 & 450 & 710 & 465\end{array}$

$\begin{array}{llllllllll}720 & & & & & & & & & \\ \text { GR } & 470 & 735 & 475 & 750 & 480 & 980 & 485 & 1010 & 495\end{array}$

$\begin{array}{llllllllll}1025 & & & & & & & & & \\ \text { GR } & 500 & 1040 & 525 & 1090 & 530 & 1110 & 530 & 1165 & 550\end{array}$

1182

$\begin{array}{llllllll}\mathrm{X} 1 & 61 & 24 & 0 & 1380 & 5280 & 5280 & 5280\end{array}$

$\begin{array}{llllllllll}\text { GR } & 565 & 0 & 550 & 20 & 525 & 55 & 520 & 58 & 515\end{array}$

82

$\begin{array}{llllllllll}\text { GR } & 500 & 112 & 485 & 135 & 480 & 550 & 475 & 650 & 470\end{array}$

$\begin{array}{llllllllll}765 & & & & & & & & & \\ \text { GR } & 464 & 790 & 470 & 820 & 475 & 830 & 480 & 835 & 485\end{array}$

$\begin{array}{llllllllll}940 & & & & & & & & & \\ \text { GR } & 500 & 1005 & 515 & 1082 & 525 & 1160 & 530 & 1200 & 535\end{array}$

1300

$\begin{array}{lllllllll}\text { GR } & 540 & 1335 & 545 & 1350 & 550 & 1360 & 565 & 1380\end{array}$

$\begin{array}{llllllll}\mathrm{X} 1 & 62 & 25 & 0 & 1625 & 5280 & 5280 & 5280\end{array}$

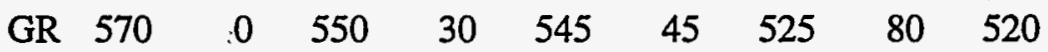

118

$\begin{array}{llllllllll}\text { GR } & 510 & 142 & 505 & 212 & 500 & 245 & 495 & 270 & 490\end{array}$

$\begin{array}{llllllllll}290 & \cdot & & & & & & & & \\ \text { GR } & 488^{\circ} & 310 & 485 & 560 & 480 & 710 & 479 & 730 & 480\end{array}$

850

$\begin{array}{llllllllll}\text { GR } & 485 & 1100 & 488 & 1200 & .490 & 1272 & 495 & 1310 & 500\end{array}$

1340

$\begin{array}{llllllllll}\text { GR } & 525 & 1378 & 530 & 1390 & 535 & 1480 & 550 & 1525 & 565\end{array}$

1625

$\begin{array}{llllllll}\mathrm{X} 1 & 63 & 20 & 0 & 1330 & 5280 & 5280 & 5280\end{array}$

$\begin{array}{llllllllll}\text { GR } & 575 & 0 & 550 & 25 & 530 & 33 & 525 & 70 & 510\end{array}$

98

$\begin{array}{llllllllll}\text { GR } & 505 & 120^{\circ} & 500 & 140 & 495 & 150 & 490 & 580 & 485\end{array}$

730

$\begin{array}{llllllllll}\text { GR } & 482 & 750 & 481 & 780 & 482 & 802 & 485 & 820 & 490 .\end{array}$

830 .

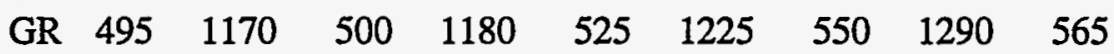
1330

$\begin{array}{llllllll}\mathrm{X} 1 & 64 & 29 & 0 & 1430 & 5280 & 5280 & 5280\end{array}$

$\begin{array}{llllllllll}\text { GR } & 575 & 0 & 560 & 20 & 555 & 40 & 550 & 55 & 545\end{array}$

75

$\begin{array}{llllllllll}\text { GR } & 540 & 87 & 535 & 110 & 525 & 120 & 515 & 140 & 510\end{array}$

160 
$\begin{array}{llllllllll}\text { GR } & 505 & 190 & 500 & 200 & 495 & 285 & 490 & 450 & 485\end{array}$

535

$\begin{array}{llllllllll}\text { GR } & 486 & 570 & 485 & 670 & 490 & 810 & 495 & 980 & 500\end{array}$

1060

$\begin{array}{llllllllll}\text { GR } & 505 & 1150 & 510 & 1230 & 515 & 1275 & 520 & 1290 & 525\end{array}$

1300

$\begin{array}{lllllllll}\text { GR } & 530 & 1310 & 535 & 1350 & 550 & 1385 & 575 & 1430\end{array}$

$\begin{array}{llllllll}\mathrm{X} 1 & 65 & 25 & 0 & 1340 & 5280 & 5280 & 5280\end{array}$

$\begin{array}{llllllllll}\text { GR } & 575 & 0 & 550 & 37 & 535 & 57 & 530 & 78 & 515\end{array}$

110

$\begin{array}{llllllllll}\text { GR } & 510 & 130 & 505 & 178 & 500 & 232 & 495 & 355 & 490\end{array}$

438

$\begin{array}{llllllllll}\text { GR } & 485 & 487 & 486 & 525 & 490 & 600 & 495 & 900 & 500\end{array}$

1045

$\begin{array}{llllllllll}\text { GR } & 505 & 1090 & 510 & 1140 & 515 & 1170 & 520 & 1190 & 525\end{array}$

1207

$\begin{array}{llllllllll}\text { GR } & 535 & 1240 & 540 & 1280 & 550 & 1290 & 570 & 1320 & 575\end{array}$.

1340

$\begin{array}{llllllll}\mathrm{X} 1 & 66 & 28 & 0 & 2555 & 5280 & 5280 & 5280\end{array}$

$\begin{array}{llllllllll}\text { GR } & 575 & 0 & 570 & 20 & 565 & 90 & 560 & 165 & 555\end{array}$

200

$\begin{array}{llllllllll}\text { GR } & 550 & 217 & 545 & 260 & 540 & 410 & 535 & 500 & 530\end{array}$

630

$\begin{array}{llllllllll}\text { GR } & 525 & 680 & 520 & 710 & 515 & 750 & 510 & 820 & 505\end{array}$

930

$\begin{array}{llllllllll}\text { GR } & 500 & 955 & 495 & 1020 & 500 & 1100 & 505 & 1140 & 509\end{array}$

$\begin{array}{llllllllll}1193 & & & & & & & & & \\ \text { GR } & 510 & 2250 & 515 & 2450 & 525 & 2470 & 540 & 2498 & 545\end{array}$

2525

$\begin{array}{lllllll}\text { GR } & 550 & 2530 & 575 & 2550 & 585 & 2555\end{array}$

$\begin{array}{llllllll}\mathrm{X} 1 & 66.5 & 31 & 0 & 3120 & 2630 & 2630 & 2630\end{array}$

$\begin{array}{llllllllll}\text { GR } & 595 & 0 & 590 & 135 & 585 & 230 & 580 & 356 & 575\end{array}$

570

$\begin{array}{llllllllll}\text { GR } & 570 & 690 & 565 & 928 & 560 & 1000 & 555 & 1250 & 550\end{array}$

1365

$\begin{array}{lllllllll}\text { GR. } 545 & 1400 & 525 & 1458 & 520 & 1480 & 515 & 1500 & 510\end{array}$

1615

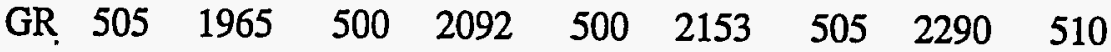
2460

$\begin{array}{llllllllll}\text { GR } & 510 & 2490 & 510 & 2880 & 515 & 2895 & 520 & 2900 & 525\end{array}$

2920

$\begin{array}{llllllllll}\text { GR } & 545 & 2950 & 550 & 2990 & 565 & 3012 & 570 & 3090 & 575\end{array}$

3100

GR $585 \quad 3120$

$\begin{array}{llllllll}\mathrm{X} 1 & 67 & 43 & 0 & 2560 & 2630 & 2630 & 2630\end{array}$

$\begin{array}{llllllllll}\text { GR } & 595 & 0 & 590 & 55 & 585 & 110 & 580 & 150 & 585\end{array}$

285 


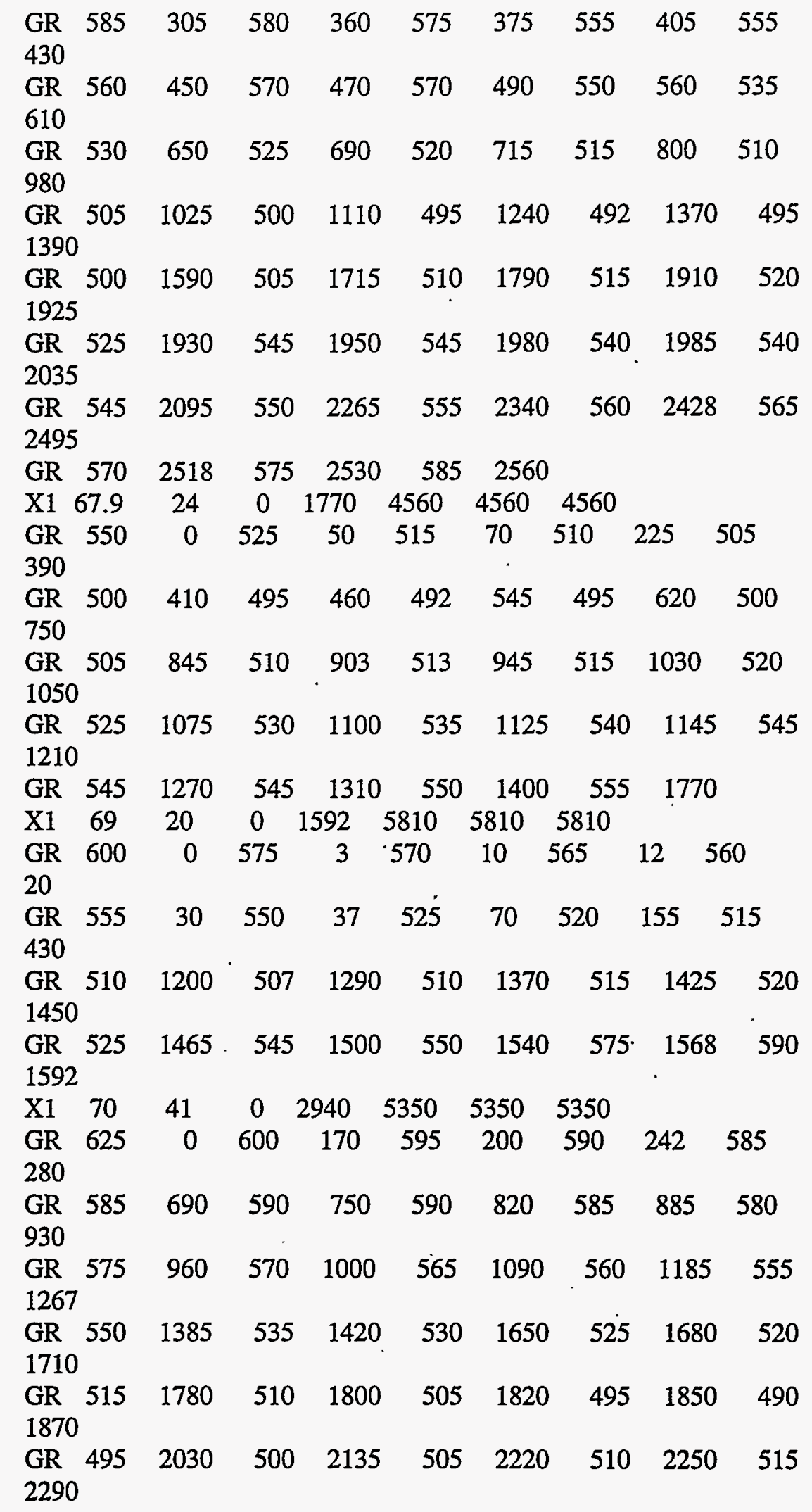




$\begin{array}{lllllllll}\text { GR 520 } & 2345 & 525 & 2380 & 525 & 2455 & 520 & 2480 & 520 \\ 2725 & & & & & & & & \\ \text { GR 525 } & 2752 & 530 & 2780 & 535 & 2825 & 550 & 2850 & 555 \\ 2880 & & & & & & & & \\ \text { GR } 600 & 2940 & & & & & & & \end{array}$.

* LITTLE GOOSE DAM. RM 70.3

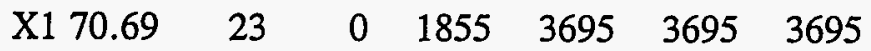

$\begin{array}{llllllllll}\text { GR } & 600 & 0 & 595 & 28 & 590 & 65 & 585 & 110 & 580\end{array}$

135

$\begin{array}{llllllllll}\text { GR } & 575 & 260 & 550 & 300 & 525 & 340 & 520 & 345 & 517.1\end{array}$

365

$\begin{array}{lllllllll}\text { GR } 511.1 & 545 & 511.1 & 628 & 517.1 & 875 & 520 & 1150 & 520\end{array}$

1585

$\begin{array}{llllllllll}\text { GR } & 525 & 1630 & 530 & 1655 & 535 & 1715 & 540 & 1730 & 550 .\end{array}$

1745

$\begin{array}{lllllll}\text { GR } & 555 & 1790 & 575 & 1815 & 600 & 1855\end{array}$

$\begin{array}{llllllll}\mathrm{X} 1 & 70.7 & 23 & 0 & 1855 & 1 & 1 & 1\end{array}$

$\begin{array}{llllllllll}\text { GR } & 600 & 0 & 595 & 28 & 590 & 65 & 585 & 110 & 580\end{array}$

135

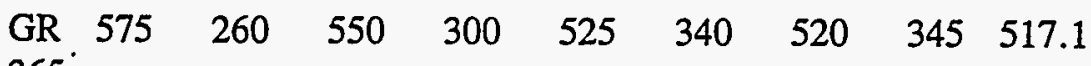

$365^{\circ}$

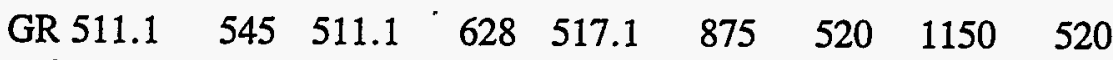
1585

$\begin{array}{llllllllll}\text { GR } & 525 & 1630 & 530 & 1655 & 535 & 1715 & 540 & 1730 & 550\end{array}$

1745

$\begin{array}{lllllll}\text { GR } & 555 & 1790 & 575 & 1815 & 600 & 1855\end{array}$

$\begin{array}{llllllll}\mathrm{X} 1 & 71 & 20 & 0 & 1610 & 1584 & 1584 & 1584\end{array}$

$\begin{array}{llllllllll}\text { GR } & 600 & 0 & 575 & 40 & 550 & 75 & 525 & 102 & 520\end{array}$

$\begin{array}{llllllllll}\text { GR } & 515 & 250 & 513 & 310 & 515 & 430 & 520 & 1225 & 525\end{array}$

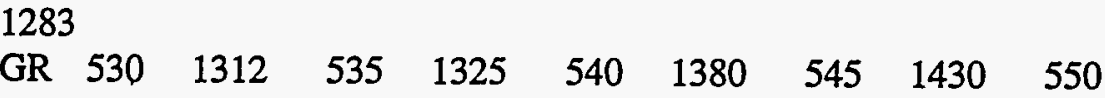

1445

$\begin{array}{llllllllll}\text { GR } & 550 & 1480 & 550 & 1507 & 555 & 1510 & 575 & 1570 & 600\end{array}$

1610

$\begin{array}{llllllll}\mathrm{X} 1 & 71.5 & 32 & 0 & 1665 & 2640 & 2640 & 2640\end{array}$

$\begin{array}{llllllllll}\text { GR } & 600 & 0 & 575 & 40 & 550 & 88 & 540 & 104 & 535\end{array}$

130

$\begin{array}{llllllllll}\text { GR } & 530 & 148 & 525 & 160 & 522 & 210 & 520 & 400 & 522\end{array}$

485

$\begin{array}{llllllllll}\text { GR } & 525 & 550 & 530 & 565 & 535 & 585 & 535 & 635 & 530\end{array}$

$\begin{array}{llllllllll}650 & & & & & & & & & \\ \text { GR } & 525 & 670 & 522 & 720 & 520 & 770 & 516 & 943 & 515\end{array}$

1025

$\begin{array}{llllllllll}\text { GR } & 515 & 1165 & 516 & 1203 & 520 & 1380 & 516 & 1415 & 525\end{array}$

1495

$\begin{array}{llllllllll}\text { GR } & 535 & 1530 & 540 & 1605 & 550 & 1620 & 555 & 1625 & 560\end{array}$ 


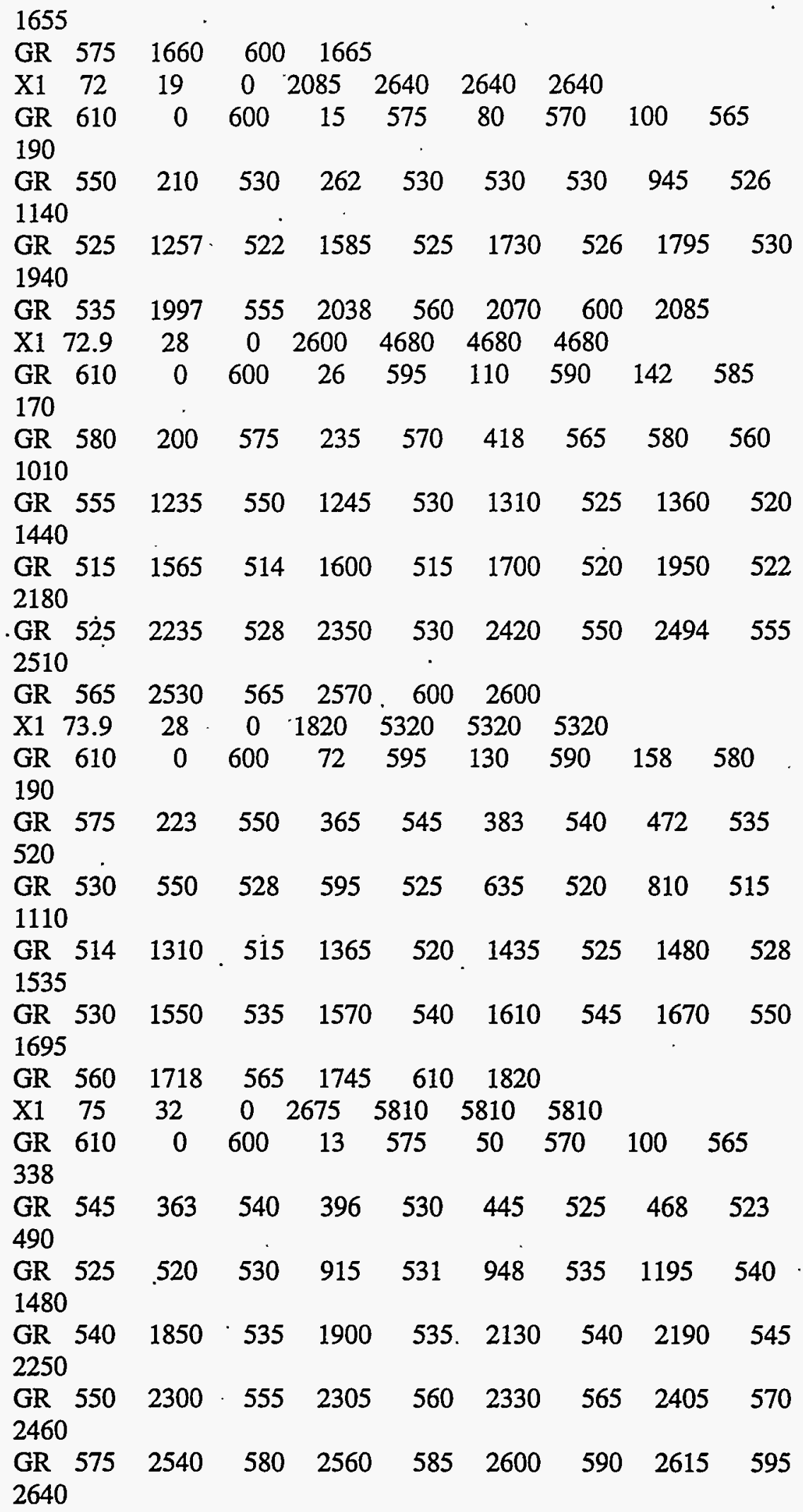


$\begin{array}{lllll}\text { GR } & 600 & 2650 & 605 & 2675\end{array}$

\begin{tabular}{|c|c|c|c|c|c|c|c|c|}
\hline $\begin{array}{ll}X 1 & 76.2\end{array}$ & 31 & 0 & 2200 & 6200 & 6200 & 6200 & & \\
\hline $\begin{array}{cc}\text { GR } & 600 \\
100 & \end{array}$ & 0 & 575 & 25 & 550 & 70 & 545 & 80 & 540 \\
\hline $\begin{array}{ll}\text { GR } & 538 \\
304 & \end{array}$ & 120 & 535 & 132 & 530 & 160 & 527 & 205 & 530 \\
\hline $\begin{array}{l}\text { GR } 532 \\
1310\end{array}$ & 400 & 532 & 1118 & 532 & 1160 & 535 & 1290 & 538 \\
\hline $\begin{array}{l}\text { GR } \quad 540 \\
1850\end{array}$ & 1335 & 545 & 1362 & 550 & 1380 & 560 & 1400 & 560 \\
\hline $\begin{array}{l}\text { GR } 555 \\
1950\end{array}$ & 1880 & 555 & 1900 & 560 & 1915 & 565 & 1925 & 565 \\
\hline $\begin{array}{l}\text { GR } 560 \\
2175\end{array}$ & 1960 & 560 & 2042 & 565 & 2070 & 575 & 2110 & 600 \\
\hline GR $\quad 620$ & 2200 & & & & & & & \\
\hline $\begin{array}{ll}X 1 & 77\end{array}$ & 28 & 0 & 2690 & 4375 & 4375 & 4375 & & \\
\hline $\begin{array}{ll}\text { GR } & 600 \\
113 & \end{array}$ & 0 & 575 & 25 & 550 & 80 & 545 & 97 & 540 \\
\hline $\begin{array}{ll}\text { GR } & 535 \\
740 & \end{array}$ & 164 & 530 & 220 & 525 & 250 & 520 & 530 & 525 \\
\hline $\begin{array}{cl}\text { GR } & 530 \\
970 & \end{array}$ & 790 & 535 & 855 & 540 & 885 & 545 & 928 & 550 \\
\hline $\begin{array}{l}\text { GR } 555 \\
2260\end{array}$ & 1020 & 560 & 1058 & 565 & 1430 & 565 & 2142 & 565 \\
\hline $\begin{array}{l}\text { GR } 565 \\
2550\end{array}$ & 2360 & 560 & 2455 & 560 & 2505 & 565 & 2525 & 570 \\
\hline GR 575 & 2570 & 600 & 2630 & 625 & 2690 & & & \\
\hline $\begin{array}{ll}X 1 & 78.1\end{array}$ & 31 & 0 & 1600 & 5990 & 5990 & 5990 & & \\
\hline $\begin{array}{ll}\text { GR } & 640 \\
190 & \end{array}$ & 0 & 635 & 125 & 630 & 140 & 625 & 150 & 600 \\
\hline $\begin{array}{cc}\text { GR } & 575 \\
321 & \end{array}$ & 240 & 570 & 253 & 565 & 267 & 560 & 285 & 555 \\
\hline $\begin{array}{ll}\text { GR } & 550 \\
705 & \end{array}$ & 345 & 545 & 380 & 540 & 435 & 539 & 488 & 535 \\
\hline $\begin{array}{l}\text { GR } 530 \\
1330\end{array}$ & 850 & 526 & 1160 & 530 & 1190 & 535 & 1220 & 539 \\
\hline $\begin{array}{l}\text { GR } 540 \\
1430\end{array}$ & 1340 & 545 & 1375 & 550 & 1398 & 555 & 1415 & 560 \\
\hline $\begin{array}{l}\text { GR } 565 \\
1570\end{array}$ & 1450 & 570 & 1468 & 575 & 1493 & 580 & 1520 & 600 \\
\hline GR $\quad 625$ & 1600 & & & & & & & \\
\hline $\begin{array}{ll}X 1 & 78.85 \\
\text { GR } & 650 \\
300 & \end{array}$ & $\begin{array}{c}29 \\
0\end{array}$ & $\begin{array}{r}0 \\
640\end{array}$ & $\begin{array}{r}1930 \\
140\end{array}$ & $\begin{array}{c}3810 \\
635\end{array}$ & $\begin{array}{l}3810 \\
205\end{array}$ & $\begin{array}{l}3810 \\
630\end{array}$ & 250 & 625 \\
\hline $\begin{array}{ll}\text { GR } & 620 \\
565 & \end{array}$ & 335 & 605 & 395 & 600 & 445 & 595 & 490 & 575 \\
\hline
\end{tabular}




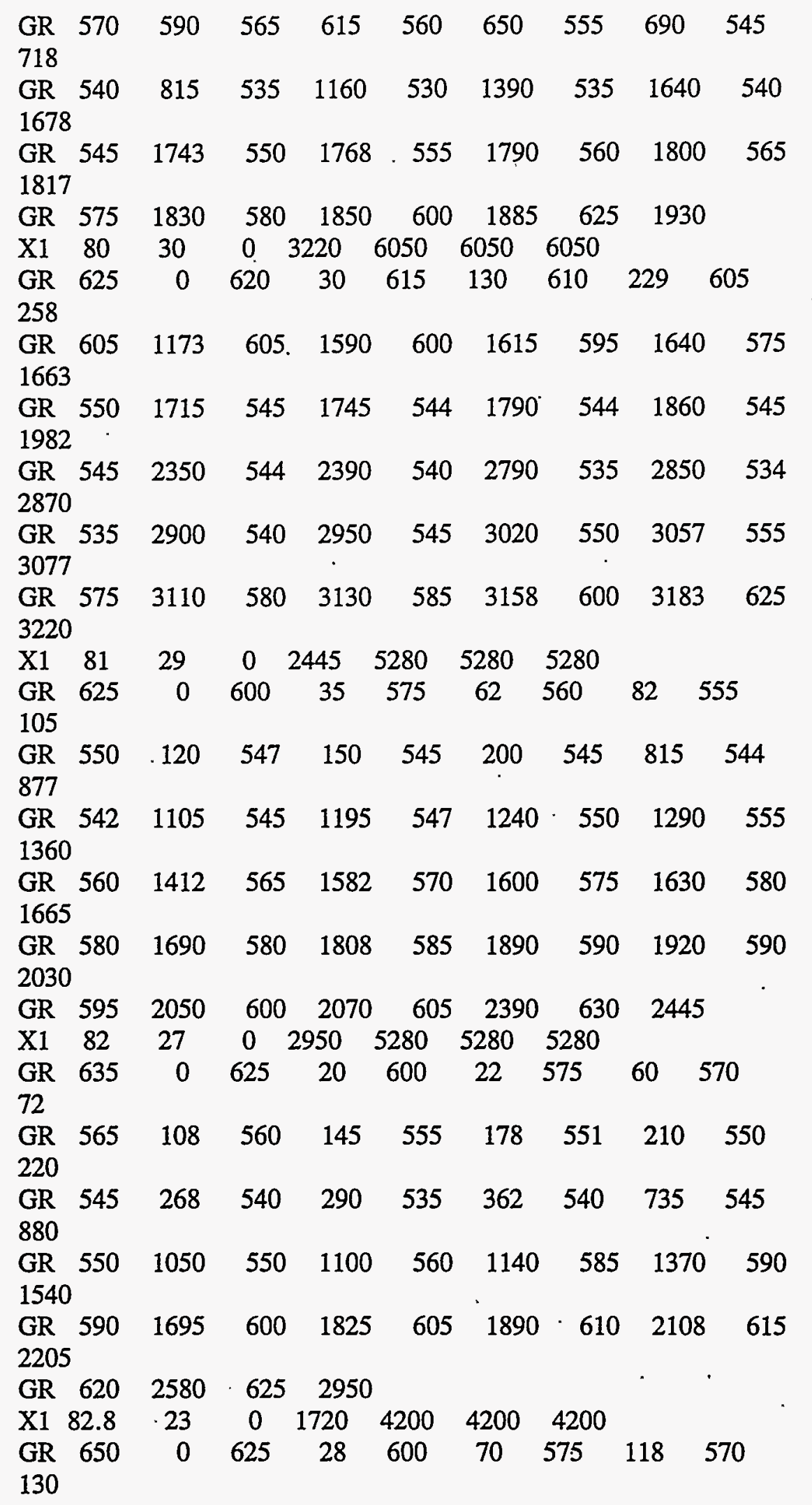


$\begin{array}{llllllllll}\text { GR } & 565 & 160 & 560 & 180 & 555 & 229 & 553 & 282 & 553\end{array}$

328

$\begin{array}{llllllllll}\text { GR } & 553 & 670 & 550 & 890 & 553 & 1388 & 550 & 1415 & 560\end{array}$

1448

$\begin{array}{llllllllll}\text { GR } & 565 & 1465 & 570 & 1490 & 575 & 1508 & 585 & 1520 & 590\end{array}$

1560

$\begin{array}{lllllll}\text { GR } & 600 & 1583 & 625 & 1643 & 630 & 1720\end{array}$

$\begin{array}{llllllll}\mathrm{X} 1 & 83.3 & 25 & 0 & 1685 & 2492 & 2492 & 2492\end{array}$

$\begin{array}{llllllllll}\text { GR } & 660 & 0 & 650 & 5 & 625 & 30 & 600 & 100 & 575\end{array}$

140

$\begin{array}{llllllllll}\text { GR } & 570 & 152 & 565 & 172 & 560 & 192 & 557 & 240 & 555\end{array}$

295

$\begin{array}{llllllllll}\text { GR } & 551 & 1180 & 551 & 1320 & 555 & 1430 & 557 & 1480 & 560\end{array}$

1502

$\begin{array}{llllllllll}\text { GR } & 565 & 1520 & 570 & 1535 & 575 & 1550 & 585 & 1560 & 590\end{array}$

1588

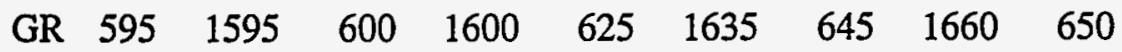
1685

$\begin{array}{llllllll}\mathrm{X} 1 & 84 & 28 & 0 & 2060 & 3910 & 3910 & 3910\end{array}$

$\begin{array}{llllllllll}\text { GR } & 645 & 0 & 625 & 30 & 600 & 90 & 590 & 105 & 585\end{array}$

122

$\begin{array}{llllllllll}\text { GR } & 580 & 895 & 575 & 910 & 570 & 945 & 565 & 965 & 560\end{array}$

987

$\begin{array}{llllllllll}\text { GR } & 557 & 1030 & 555 & 1075 & 550 & 1100 & 545 & 1130 & 540\end{array}$

1312

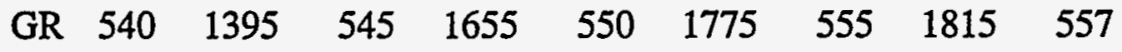

1840

$\begin{array}{llllllllll}\text { GR } & 560 & 1850 & 565 & 1882 & 570 & 1905 & 575 & 1930 & 580\end{array}$

1935

$\begin{array}{lllllll}\text { GR } & 585 & 1950 & 590 & 1978 & 635 & 2060\end{array}$

$\begin{array}{llllllll}\mathrm{X} 1 & 85 & 29 & 0 & 1840 & 5280 & 5280 & 5280\end{array}$

$\begin{array}{llllllllll}\text { GR } & 635 & 0 & 630 & 38 & 625 & 75 & 620 & 95 & 615\end{array}$

110

$\begin{array}{llllllllll}\text { GR } & 610 & 130 & 605 & 210 & 600 & 278 & 590 & 295 & 585\end{array}$

330

$\begin{array}{llllllllll}\text { GR } & 580 & 367 & 575 & 395 & 570 & 430 & 565 & 450 & 560\end{array}$

480

$\begin{array}{lllllllll}\text { GR } 558.6 & 520 & 552.6 & 632 & 550.2 & 740 & 552.6 & 1350 & 555\end{array}$

1410

$\begin{array}{llllllllll}\text { GR } & 560 & 1540 & 575 & 1564 & 585 & 1587 & 590 & 1610 & 600\end{array}$

1620

$\begin{array}{lllllllll}\text { GR } & 625 & 1700 & 630 & 1720 & 635 & 1780 & 640 & 1840\end{array}$

$\begin{array}{llllllll}\mathrm{X} 1 & 86 & 39 & 0 & 2310 & 5280 & 5280 & 5280\end{array}$

$\begin{array}{llllllllll}\text { GR } & 640 & 0 & 635 & 20 & 625 & 40 & 600 & 82 & 585\end{array}$

108 .

$\begin{array}{llllllllll}\text { GR } & 580 & 150 & 575 & 167 & 570 & 180 & 565 & 200 & 560\end{array}$

239 


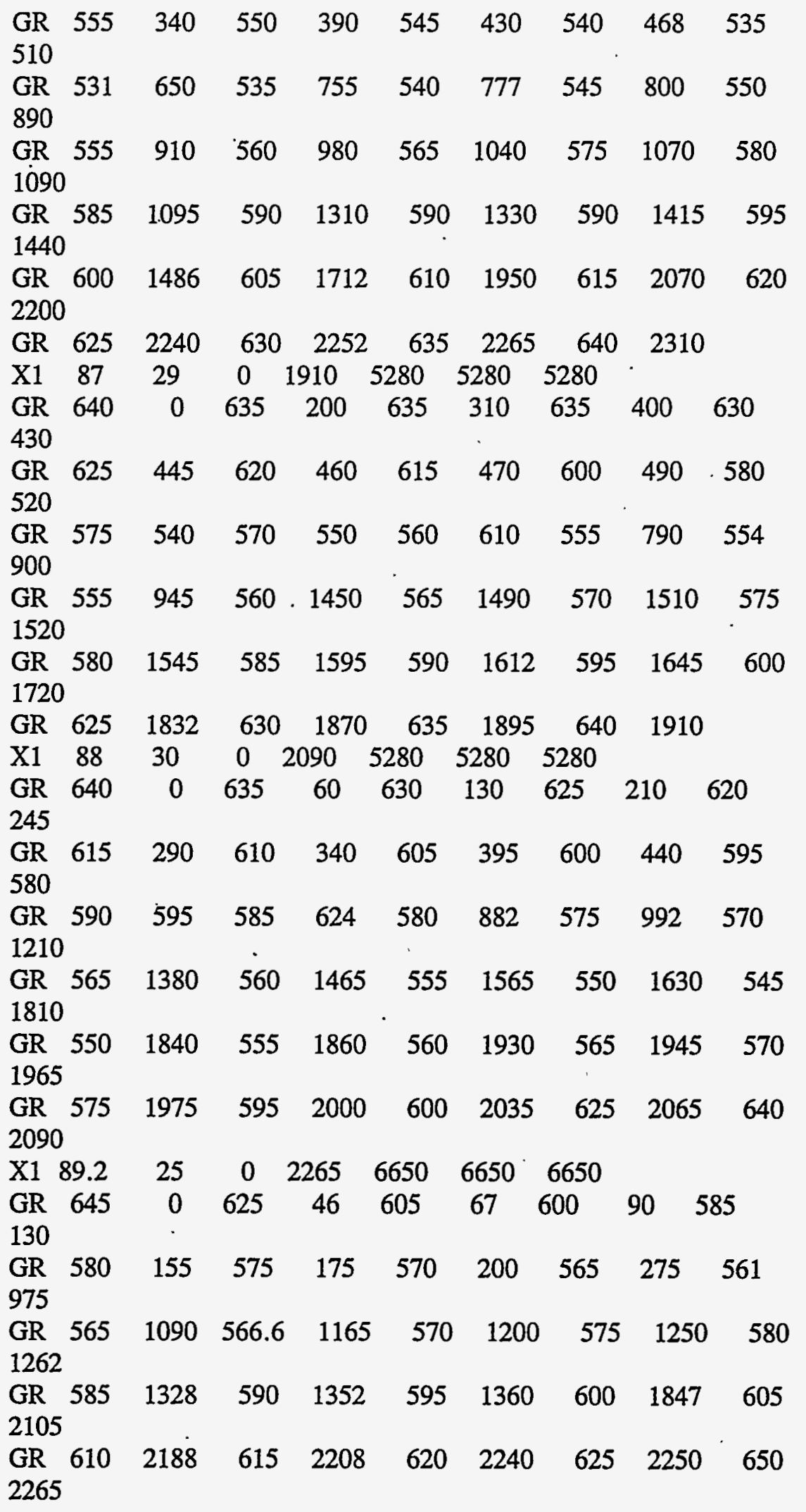




\begin{tabular}{|c|c|c|c|c|c|c|c|c|c|}
\hline $\mathrm{X} 1$ & 90 & 27 & 0 & 2482 & 3880 & 3880 & 3880 & & \\
\hline GR & 640 & 0 & 625 & 20 & 600 & 68 & 595 & 88 & 580 \\
\hline 127 & & & & & & & & & \\
\hline $\begin{array}{l}\text { GR } \\
970\end{array}$ & 575 & 150 & 570 & 200 & 565 & 296 & 560 & 835 & 565 \\
\hline $\begin{array}{l}\text { GR } \\
1535\end{array}$ & 570 & 1120 & 575 & 1150 & 595 & 1250 & 600 & 1405 & 605 \\
\hline $\begin{array}{l}\text { GR } \\
2110\end{array}$ & 605 & 1570 & 600 & 1715 & 605 & 1925 & 610 & 1980 & 610 \\
\hline $\begin{array}{l}\text { GR } \\
2438\end{array}$ & 600 & 2160 & 605 & 2200 & 610 & 2220 & 615 & 2380 & 620 \\
\hline GR. & 625 & 2448 & 650 & 2482 & & & & & \\
\hline $\mathrm{X} 1$ & 91 & 28 & 0 & 2480 & 5280 & 5280 & 5280 & & \\
\hline $\begin{array}{l}\text { GR } \\
130\end{array}$ & 650 & 0 & 625 & 35 & 600 & 70 & 580 & 105 & 575 \\
\hline $\begin{array}{l}\text { GR } \\
420\end{array}$ & 575 & 180 & 570 & 250 & 565 & $\cdot 270$ & 560 & 330 & 559 \\
\hline $\begin{array}{l}\text { GR } \\
1010\end{array}$ & 559 & 500 & 560 & 525 & 565 & 660 & 570 & 865 & 575 \\
\hline $\begin{array}{l}\text { GR } \\
1280\end{array}$ & 580 & 1100 & 585 & 1185 & 590 & 1230 & 595 & 1255 & 610 \\
\hline $\begin{array}{l}\text { GR } \\
2337\end{array}$ & 7615 & 2040 & 620 & 2100 & 625 & 2200 & 630 & 2285 & 635 \\
\hline GR & 640 & 2390 & 645 & 2455 & 650 & 2480 & & & \\
\hline $\mathrm{X} 1$ & 92.1 & 32 & 0 & 2132 & 5950 & $\begin{array}{c}5950 \\
32\end{array}$ & 5950 & & \\
\hline $\begin{array}{l}\text { GR } \\
150\end{array}$ & 655 & 0 & 650 & 30 & 645 & 70 & 645 & 125 & 650 \\
\hline $\begin{array}{l}\text { GR } \\
500\end{array}$ & 655 & 310 & 650 & 370 & 645 & 405 & 625 & 470 & 615 \\
\hline $\begin{array}{l}\text { GR } \\
660\end{array}$ & 610 & 550 & 600 & 565 & 595 & 590 & 590 & 630 & 585 \\
\hline $\begin{array}{l}\text { GR } \\
1630\end{array}$ & 580 & 740 & 575 & 1095 & 570 & 1390 & 569 & 1600 & 570 \\
\hline $\begin{array}{l}\text { GR } \\
1920\end{array}$ & 575 & 1760 & 580 & 1860 & 585 & 1870 & 590 & 1900 & 595 \\
\hline $\begin{array}{l}\text { GR } \\
2045\end{array}$ & 600 & 1950 & 605 & 1958 & 610 & 1990 & 615 & 2000 & 620 \\
\hline GR & 650 & 2110 & 655 & 2132 & & & & & \\
\hline $\mathrm{X} 1$ & 93 & 32 & 0 & 1970 & 4545 & 4545 & 4545 & & \\
\hline $\begin{array}{l}\text { GR } \\
500\end{array}$ & 660 & 0 & 655 & 80 & 650 & 190 & 645 & 320 & 640 \\
\hline $\begin{array}{l}\text { GR } \\
710\end{array}$ & 635 & 600 & 630 & 640 & 625 & 677 & 620 & 700 & 615 \\
\hline $\begin{array}{l}\text { GR } \\
945\end{array}$ & 610 & 780 & 600 & 800 & 585 & 870 & 580 & 900 & 575 \\
\hline GR & 570 & 1060 & 565 & 1270 & 564 & 1425 & 565 & 1480 & 570 \\
\hline
\end{tabular}


1530

$\begin{array}{llllllllll}\text { GR } & 575 & 1590 & 580 & 1700 & 585 & 1730 & 590 & 1760 & 595\end{array}$ 1800

$\begin{array}{llllllllll}\text { GR } & 600 & 1835 & 605 & 1840 & 610 & 1870 & 615 & 1905 & 615\end{array}$ 1920

GR $\quad 620 \quad 1920 \quad 655 \quad 1970$

$\begin{array}{llllllll}\mathrm{X} 1 & 94 & 32 & 0 & 2410 & 5345 & 5345 & 5345\end{array}$

$\begin{array}{llllllllll}\text { GR } & 660 & 0 & 655 & 15 & 635 & 240 & 630 & 350 & 625\end{array}$

392

$\begin{array}{llllllllll}\text { GR } & 620 & 440 & 615 & 475 & 610 & 530 & 605 & 540 & 600\end{array}$

550

$\begin{array}{llllllllll}\text { GR } & 595 & 560 & 590 & \cdot 630 & 585 & 695 & 580 & 1140 & 575\end{array}$

1285

$\begin{array}{llllllllll}\text { GR } & 573 & 1375 & 573 & 1485 & 575 & 1540 & 580 & 1660 & 585\end{array}$

1760

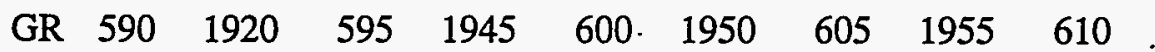

1980

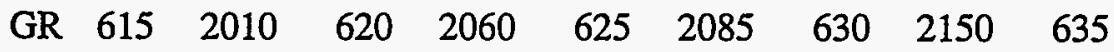

2190

GR $\quad 640 \quad 2250 \quad 660 \quad 2410$

$\begin{array}{llllllll}\mathrm{X} 1 & 95 & 23 & 0 & 2695 & 5280 & 5280 & 5280\end{array}$

$\begin{array}{llllllllll}\text { GR } & 665 & 0 & 650 & 30 & 625 & 71 & 605 & 108 & 600\end{array}$

$\cdot 131$

$\begin{array}{llllllllll}\text { GR } & 585 & 180 & 580 & 230 & 575 & 253 & 570 & 350 & 575\end{array}$

568

$\begin{array}{llllllllll}\text { GR } & 580 & 865 & 585 & 1100 & 600 & 1146 & 620 & 1180 & 625\end{array}$

1225

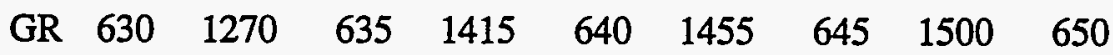

1605

$\begin{array}{lllllll}\text { GR } & 655 & 2380 & 660 & 2550 & 665 & 2695\end{array}$

$\begin{array}{llllllll}\mathrm{X} 1 & 96 & 34 & 0 & 2550 & 5280 & 5280 & 5280\end{array}$

$\begin{array}{llllllllll}\text { GR } & 665 & 0 & 650 & 35 & 630 & 65^{*} & 625 & 72 & 600\end{array}$

135

$\begin{array}{llllllllll}\text { GR } & 585 & 178 & 583.5 & 200 & 580 & 230 & 575 & 337 & 570\end{array}$

$\begin{array}{lllllllllll}378 & & & & & & & & & \\ \text { GR } & 565 & 508 & 570 & 727 & 575 & 778 & 580 & 855 & 585\end{array}$

$\begin{array}{llllllllll}922 & & & & & & & & & \end{array}$

1115

$\begin{array}{llllllllll}\text { GR } & 615 & 1170 & 620 & 1195 & 620 & 1250 & 615 & 1282 & 615\end{array}$.

1340

$\begin{array}{llllllllll}\text { GR } & 640 & 1390 & 645 & 1645 & 645 & 1800 & 645 & 2100 & 650\end{array}$

2195

$\begin{array}{lllllllll}\text { GR } & 655 & 2295 & 660 & 2375 & 665 & 2480 & 670 & 2550\end{array}$

$\begin{array}{llllllll}\mathrm{X} 1 & 96.9 & 24 & 0 & 1975 & 4560 & 4560 & 4560\end{array}$

$\begin{array}{llllllllll}\text { GR } & 675 & 0 & 650 & 20 & 625 & 50 & 600 & 100 & 595\end{array}$

120 
$\begin{array}{llllllllll}\text { GR } & 590 & 140 & 585 & 175 & 580 & 420 & 576 & 565 & 580\end{array}$

900

$\begin{array}{llllllllll}\text { GR } & 585 & 962 & 590 & 995 & 595 & 1060 & 595 & 1260 & 595\end{array}$

1410

$\begin{array}{llllllllll}\text { GR } & 600 & 1440 & 605 & 1470 & 620 & 1500 & 625 & 1580 & 630\end{array}$

1610

$\begin{array}{lllllllll}\text { GR } & 635 & 1720 & 640 & 1870 & 650 & 1900 & 670 & 1975\end{array}$

$\begin{array}{llllllll}\mathrm{X} 1 & 98 & 30 & 0 & 2265 & 5920 & 5920 & 5920\end{array}$

$\begin{array}{llllllllll}\text { GR } & 670 & 0 & 665 & 40 & 660 & 65 & 655 & 125 & 650\end{array}$

188

$\begin{array}{llllllllll}\text { GR } & 645 & 250 & 640 & 320 & 635 & 445 & 630 & 810 & 625\end{array}$

920

$\begin{array}{llllllllll}\text { GR } & 620 & 1010 & 615 & 1095 & 610 & 1115 & 605 & 1150 & 600\end{array}$

1195

$\begin{array}{llllllllll}\text { GR } & 595 & 1220 & 590 & 1268 & 585 & 1390 & 580 & 1410 & 575\end{array}$

1540

$\begin{array}{llllllllll}\text { GR } & 573 & 1715 & 575 & 1850 & 580 & 1900 & 585 & 1940 & 590\end{array}$

2060

$\begin{array}{llllllllll}\text { GR } & 600 & 2080 & 625 & 2150 & 630 & 2200 & 650 & 2235 & 670\end{array}$ 2265

$\begin{array}{llrrrrrrrr}\mathrm{X} 1 & 99 & 35 & 0 & 2250 & 5380 & 5380 & 5380 & & . \\ \mathrm{GR} & 670 & 0 & 665 & 20 & 660 & 100 & 655 & 330 & 650\end{array}$

390

$\begin{array}{llllllllll}\text { GR } & 635 & 500 & 630 & 530 & 625 & 540 & 610 & 590 & 605 .\end{array}$

617

$\begin{array}{llllllllll}\text { GR } & 605 & 720 & 610 & 740 & 615 & 818 & 620 & 983 & 620\end{array}$

1100

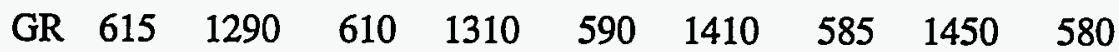

1490

$\begin{array}{llllllllll}\text { GR } & 575 & 1530 & 570 & 1660 & 575 & 1738 & 580 & 1760 & 585\end{array}$

1900

$\begin{array}{llllllllll}\text { GR } & 590 & 2000 & 595 & 2040 & 600 & 2060 & 605 & 2080 & 610\end{array}$ 2100

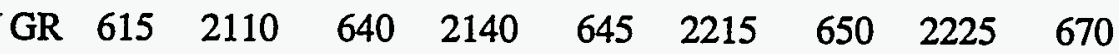
2250

$\begin{array}{llllllll}\mathrm{X} 1 & 100 & 27 & 0 & 2405 & 5280 & 5280 & 5280\end{array}$

$\begin{array}{llllllllll}\text { GR } & 675 & 0 & 655 & 50 & 650 & 80 & 625 & 150 & 605\end{array}$

238

$\begin{array}{llllllllll}\text { GR } & 600 & 275 & 595 & 340 & 590 & 705 & 588 & 928 & 590\end{array}$

$\begin{array}{llllllllll}1075 & & & & & & & & & \\ \text { GR } & 595 & 1190 & 600 & 1255 & 605 & 1310 & 610 & 1360 & 615\end{array}$

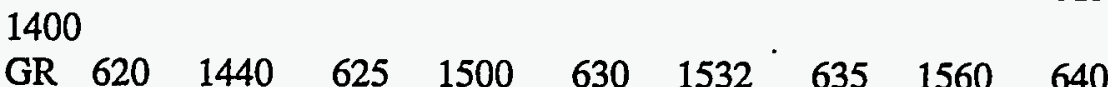

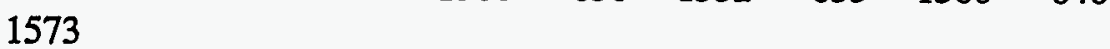

$\begin{array}{llllllllll}\text { GR } & 645 & 1755 & 650 & 1935 & 655 & 2070 & 660 & 2125 & 665\end{array}$ 2220

GR. $670 \quad 2303 \quad 675 \quad 2405$ 
$\begin{array}{lllllllll}\mathrm{X} 1101.87 & .81 & 500.0 & 3562.0 & 9874 & 9874 & 9874 & .0000 & .00\end{array}$

$\begin{array}{lllllllll}\text { GR } 653.9 & 500.0 & 634.0 & 554.2 & 634.5 & 645.0 & 634.2 & 720.6 & 634.2\end{array}$

770.3

$\begin{array}{lllllllll}\text { GR } 633.7 & 819.4 & 632.8 & 885.9 & 632.7 & 9 \dot{4} 2.7 & 625.9 & 997.6 & 625.0\end{array}$ 1042.3

GR. $626.0 \quad 1089.4 \quad 626.3 \quad 1136.2 \quad 626.3 \quad 1179.1 \quad 626.2 \quad 1226.6 \quad 625.5$ 1274.6

$\begin{array}{lllllllll}\text { GR } 625.4 & 1317.4 & 625.0 & 1360.4 & 624.9 & 1401.1 & 624.0 & 1452.5 & 623.8\end{array}$ 1494.6

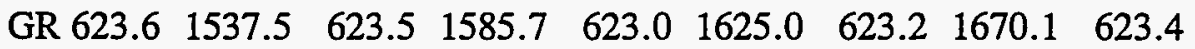
1708.4

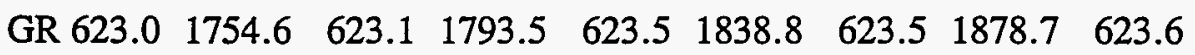
1923.5

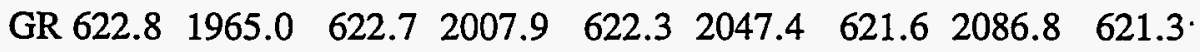
2131.1 .

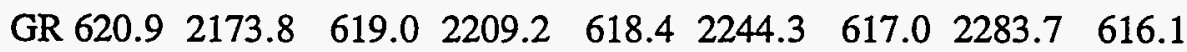
2323.0

GR 615.2 $2354.2 \quad 614.7 \quad 2389.6 \quad 614.1 \quad 2419.6 \quad 613.7 \quad 2456.3 \quad 612.7$ 2490.2

$\begin{array}{lllllllll}\text { GR } 611.2 & 2522.6 & 606.7 & 2552.3 & 601.9 & 2580.7 & 598.0 & 2606.8 & 600.5\end{array}$ 2632.8

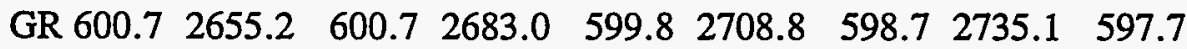
2758.0

$\begin{array}{lllllllll}\text { GR } 596.8 & 2780.4 & 595.8 & 2807.1 & 595.4 & 2832.2 & 597.7 & 2857.2 & 600.4\end{array}$ 2882.3

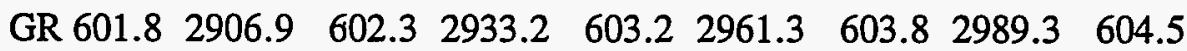
3015.4

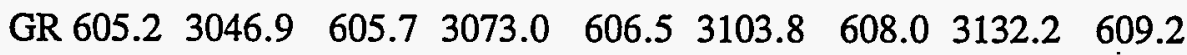
3160.7

$\begin{array}{lllllllll}\text { GR } 609.8 & 3188.3 & 609.0 & 3217.0 & 609.6 & 3252.6 & 609.7 & 3287.9 & 609.5\end{array}$ 3315.0

$\begin{array}{lllllllll}\text { GR } 618.7 & 3352.4 & 617.6 & 3384.9 & 617.2 & 3421.4 & 618.1 & 3454.8 & 625.8\end{array}$ 3503.7

GR 647.13562 .0

$\begin{array}{lllllllll}\mathrm{X} 1102.06 & 81 & 500.0 & 2981.8 & 1003 & 1003 & 1003 & .0000 & .00\end{array}$ $\begin{array}{lllllllll}\text { GR } 670.8 & 500.0 & 646.5 & 592.5 & 638.6 & 651.3 & 637.2 & 691.2 & 635.8\end{array}$ 747.6

$\begin{array}{lllllllll}\text { GR } 635.3 & 799.8 & 635.2 & 830.5 & 634.9 & 880.2 & 634.5 & 921.0 & 634.1\end{array}$ 957.2

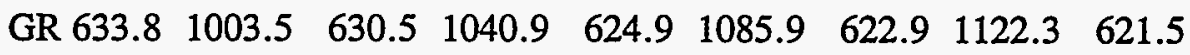
1153.4

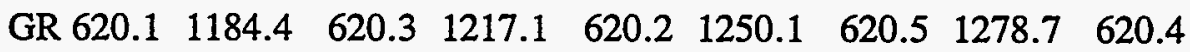
1311.4

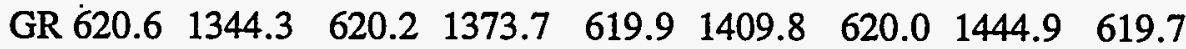
1473.0

$\begin{array}{lllllllll}\text { GR } 618.8 & 1501.3 & 615.2 & 1535.6 & 614.7 & 1567.3 & 613.3 & 1595.0 & 612.0\end{array}$ 1622.1 
$\begin{array}{lllllllll}\text { GR } 609.5 & 1649.4 & 608.9 & 1677.0 & 607.4 & 1702.6 & 607.9 & 1728.2 & 606.8\end{array}$ 1755.9

$\begin{array}{lllllllll}\text { GR } 606.9 & 1782.2 & 607.9 & 1804.6 & 608.8 & 1832.9 & 609.6 & 1860.4 & 610.2\end{array}$ 1885.4

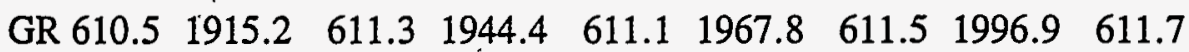
2025.9

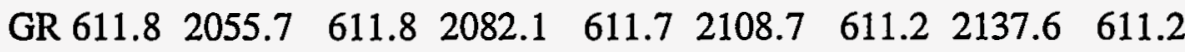
2166.3

$\begin{array}{lllllllll}\text { GR } 610.3 & 2189.2 & 610.0 & 2218.6 & 608.8 & 2246.1 & 607.8 & 2272.2 & 607.5\end{array}$ 2295.8

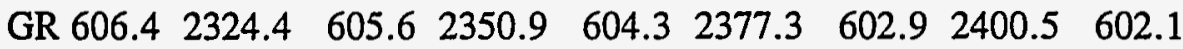
2424.3

$\begin{array}{lllllllll}\text { GR } 600.9 & 2447.9 & 600.8 & 2470.8 & 600.3 & 2494.4 & 599.7 & 2518.6 & 599.3\end{array}$ 2542.5

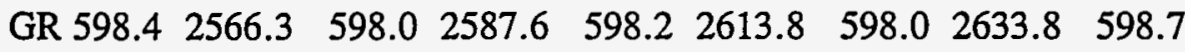
2661.0

$\begin{array}{lllllllll}\text { GR 599.1 } & 2681.2 & 601.5 & 2707.7 & 601.8 & 2730.1 & 601.1 & 2752.2 & 608.9\end{array}$ 2779.5

$\begin{array}{lllllllll}\text { GR } 616.2 & 2811.3 & 614.3 & 2835.6 & 615.2 & 2864.8 & 619.9 & 2895.3 & 636.8\end{array}$ 2935.6

GR 644.42981 .8

$\begin{array}{lllllllll}\mathrm{X} 1102.25 & 81 & 500.0 & 2586.9 & 1003 & 1003 & 1003 & .0000 & .00\end{array}$

$\begin{array}{lllllllll}\text { GR } 668.2 & 500.0 & 653.7 & 575.5 & 645.8 & 661.5 & 647.8 & 726.0 & 649.3\end{array}$

780.6

$\begin{array}{lllllllll}\text { GR } 649.4 & 834.7 & 635.9 & 886.9 & 635.2 & 918.8 & 635.2 & 965.3 & 634.6\end{array}$

1023.2

$\begin{array}{lllllllll}\text { GR } 633.3 & 1043.4 & 629.6 & 1080.6 & 627.3 & 1111.6 & 624.9 & 1141.6 & 623.1\end{array}$ 1169.9

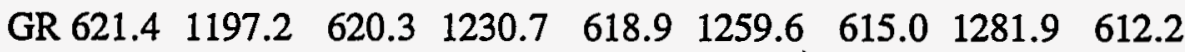
1309.4

$\begin{array}{lllllllll}\text { GR } 609.6 & 1332.6 & 609.6 & 1359.5 & 608.1 & 1378.8 & 605.9 & 1404.8 & 604.1\end{array}$ 1422.9

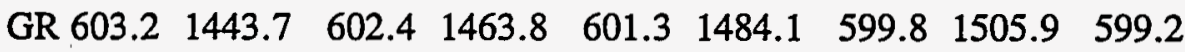
1528.0

$\begin{array}{lllllllll}\text { GR } 599.9 & 1545.9 & 599.8 & 1567.3 & 600.2 & 1586.8 & 600.5 & 1606.0 & 600.8\end{array}$ 1627.6

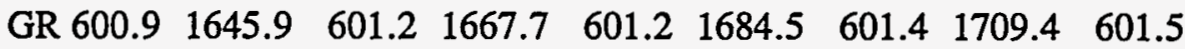
1728.7

$\begin{array}{lllllllll}\text { GR.601.9 } & 1750.7 & 601.9 & 1769.7 & 602.2 & 1792.0 & 602.6 & 1818.5 & 602.7\end{array}$

1834.3

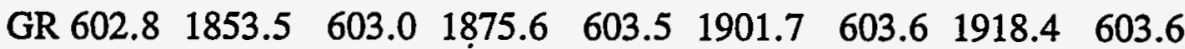
1942.9

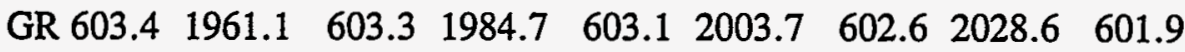
2045.3

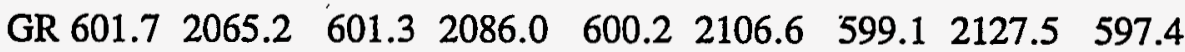
2150.0

$\begin{array}{lllllllll}\text { GR } 597.2 & 2166.3 & 596.8 & 2187.9 & 595.8 & 2205.5 & 594.1 & 2226.0 & 593.0\end{array}$ 
2241.7

$\begin{array}{lllllllll}\text { GR } 591.8 & 2257.4 & 592.1 & 2279.0 & 592.0 & 2299.8 & 592.2 & 2315.3 & 591.5\end{array}$ 2331.6

$\begin{array}{lllllllll}\text { GR } 591.3 & 2351.1 & 592.1 & 2370.2 & 590.2 & 2388.0 & 588.5 & 2407.6 & 590.2\end{array}$ 2423.0

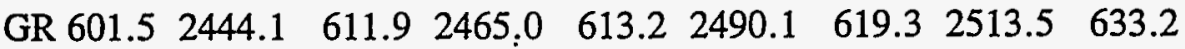
2550.7

GR 645.62586 .9

$\begin{array}{lllllllll}\mathrm{X} 1102.44 & 81 & 500.0 & 1916.0 & 1003 & 1003 & 1003 & .0000 & .00\end{array}$

$\begin{array}{lllllllll}\text { GR } 651.1 & 500.0 & 635.7 & 557.2 & 633.1 & 605.5 & 625.2 & 630.6 & 621.3\end{array}$

659.3

$\begin{array}{lllllllll}\text { GR } 619.7 & 680.8 & 617.1 & 709.1 & 614.7 & 729.7 & 612.1 & 749.5 & 611.4\end{array}$

770.1

$\begin{array}{lllllllll}\text { GR } 608.2 & 786.5 & 605.8 & 803.6 & 605.7 & 822.9 & 605.2 & 836.4 & 604.7\end{array}$

855.1

$\begin{array}{lllllllll}\text { GR } 604.0 & 867.7 & 603.4 & 889.7 & 602.9 & 909.9 & 602.4 & 921.6 & 602.2\end{array}$

935.9

$\begin{array}{lllllllll}\text { GR } 601.9 & 952.9 & 601.5 & 968.8 & 601.4 & 984.9 & 601.2 & 1000.4 & 600.5\end{array}$ 1016.1

$\begin{array}{lllllllll}\text { GR } 600.8 & 1032.8 & 600.3 & 1047.5 & 600.4 & 1063.9 & 600.0 & 1078.7 & 600.2\end{array}$ 1094.5

$\begin{array}{lllllllll}\text { GR } 599.9 & 1108.4 & 599.9 & 1121.9 & 599.8 & 1139.5 & 599.9 & 1153.6 & 599.8\end{array}$ 1168.6

$\begin{array}{lllllllll}\text { GR } 599.8 & 1187.3 & 599.9 & 1200.6 & 599.7 & 1218.1 & 599.4 & 1230.2 & 599.3\end{array}$ 1248.6

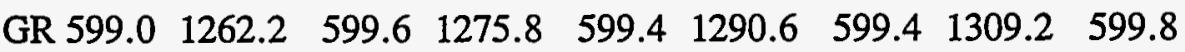
1322.2

$\begin{array}{lllllllll}\text { GR } 599.7 & 1340.9 & 599.8 & 1352.6 & 600.0 & 1371.7 & 599.7 & 1383.5 & 599.9\end{array}$ 1399.9

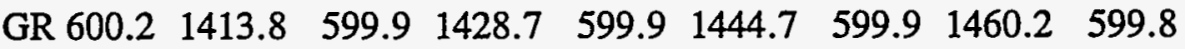
1473.9

$\begin{array}{lllllllll}\text { GR } 599.8 & 1486.9 & 599.4 & 1504.9 & 599.1 & 1517.2 & 598.7 & 1535.5 & 598.3\end{array}$ 1548.7

$\begin{array}{lllllllll}\text { GR } 597.1 & 1564.8 & 596.4 & 1579.4 & 596.1 & 1594.6 & 595.7 & 1610.1 & 594.9\end{array}$ 1623.4

$\begin{array}{lllllllll}\text { GR } 594.0 & 1637.5 & 593.5 & 1650.5 & 594.6 & 1662.9 & 594.2 & 1678.3 & 594.5\end{array}$ 1693.9

$\begin{array}{lllllllll}\text { GR } 594.4 & 1706.8 & 595.2 & 1719.7 & 594.9 & 1733.6 & 596.0 & 1746.0 & 597.8\end{array}$ 1763.8

$\begin{array}{lllllllll}\text { GR } 604.3 & 1780.6 & 613.9 & 1797.7 & 618.4 & 1822.7 & 621.3 & 1842.0 & 635.5\end{array}$ 1876.2

GR 639.51916 .0

$\begin{array}{lllllllll}\mathrm{X} 1102.63 & 81 & 500.0 & 1772.8 & 1003 & 1003 & 1003 & .0000 & .00\end{array}$

$\begin{array}{lllllllll}\text { GR } 642.3 & 500.0 & 637.5 & 546.2 & 627.0 & 587.0 & 620.9 & 613.9 & 621.2\end{array}$

635.7

$\begin{array}{lllllllll}\text { GR } 619.3 & 656.3 & 616.3 & 679.2 & 614.9 & 697.4 & 613.7 & 716.2 & 610.7\end{array}$ 735.3 
$\begin{array}{lllllllll}\text { GR } 608.2 & 752.3 & 605.6 & 768.2 & 604.6 & 783.3 & 604.0 & 801.8 & 603.4\end{array}$ 814.4

$\begin{array}{lllllllll}\text { GR } 602.7 & 829.8 & 602.8 & 843.2 & 602.8 & 857.4 & 602.4 & 873.8 & 602.1\end{array}$ 889.9

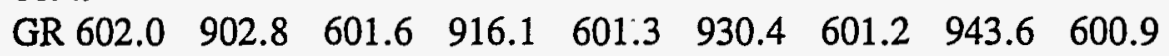
959.9

$\begin{array}{lllllllll}\text { GR } 600.7^{*} & 970.6 & 600.3 & 988.5 & 599.9 & 1006.2 & 599.8 & 1015.8 & 599.4\end{array}$ 1030.1

$\begin{array}{lllllllll}\text { GR } 599.3 & 1043.2 & 599.1 & 1056.8 & 598.5 & 1071.0 & 598.1 & 1084.4 & 598.1\end{array}$ 1097.7

$\begin{array}{lllllllll}\text { GR } 598.0 & 1113.1 & 598.1 & 1125.3 & 598.3 & 1139.5 & 598.3 & 1151.1 & 598.1\end{array}$ 1164.4

$\begin{array}{lllllllll}\text { GR } 597.7 & 1178.8 & 597.7 & 1192.1 & 597.4 & 1205.1 & 596.9 & 1215.9 & 597.1\end{array}$ 1231.5

$\begin{array}{lllllllll}\text { GR } 597.1 & 1242.9 & 596.6 & 1259.3 & 596.5 & 1272.0 & 596.1 & 1284.7 & 596.3\end{array}$ 1297.5

$\begin{array}{lllllllll}\text { GR 596.3 } & 1311.3 & 596.1 & 1324.4 & 595.8 & 1336.4 & 595.5 & 1350.7 & 595.6\end{array}$ 1361.8

$\begin{array}{lllllllll}\text { GR } 595.8 & 1376.5 & 596.0 & 1389.1 & 596.3 & 1402.0 & 596.5 & 1414.9 & 596.8\end{array}$ 1427.7

$\begin{array}{lllllllll}\text { GR } 596.8 & 1439.9 & 596.3 & 1457.1 & 596.1 & 1467.5 & 595.8 & 1483.2 & 595.6\end{array}$ 1493.4

$\begin{array}{lllllllll}\text { GR } 594.8 & 1508.3 & 594.6 & 1519.0 & 595.1 & 1533.7 & 595.3 & 1543.6 & 594.7\end{array}$ 1558.1

$\begin{array}{lllllllll}\text { GR } 594.5 & 1570.4 & 593.9 & 1582.0 & 593.8 & 1595.1 & 593.8 & 1611.7 & 595.8\end{array}$ 1619.5

$\begin{array}{lllllllll}\text { GR 595.5 } & 1629.9 & 604.9 & 1647.9 & 617.8 & 1669.4 & 625.8 & 1686.0 & 634.5\end{array}$ 1726.9

GR 648.71772 .8

$\begin{array}{lllllllll}\mathrm{X} 1102.80 & 81 & 500.0 & 1709.7 & 899 & 899 & 899 & .0000 & .00\end{array}$ $\begin{array}{lllllllll}\text { GR } 642.3 & 500.0 & 637.1 & 549.5 & 631.1 & 597.5 & 623.4 & 620.4 & 622.9\end{array}$ 650.4

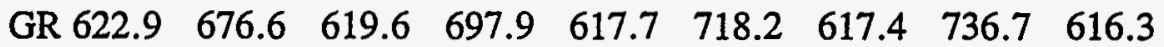

756.0

$\begin{array}{lllllllll}\text { GR } 613.8 & 775.4 & 612.3 & 788.9 & 608.6 & 811.0 & 606.4 & 830.4 & 605.2\end{array}$ 840.6

$\begin{array}{lllllllll}\text { GR } 604.1 & 854.7 & 603.9 & 869.6 & 603.6 & 885.0 & 603.3 & 897.9 & 603.1\end{array}$

911.8

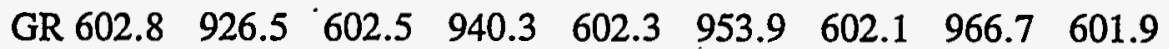
980.4

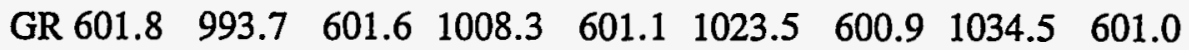
1046.9

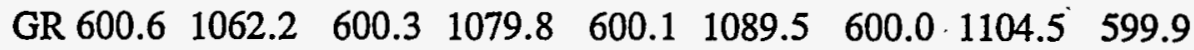
1115.2

$\begin{array}{lllllllll}\text { GR } 599.4 & 1129.2 & 598.8 & 1141.3 & 598.6 & 1153.5 & 598.2 & 1165.8 & 597.7\end{array}$ 1179.2

$\begin{array}{lllllllll}\text { GR } 597.2 & 1191.1 & 596.9 & 1203.9 & 596.6 & 1216.5 & 596.3 & 1229.9 & 595.7\end{array}$ 
1240.5

$\begin{array}{llllllll}\text { GR 595.4· } 1250.1 & 595.2 & 1264.9 & 594.6 & 1277.0 & 594.3 & 1288.8 & 593.8\end{array}$ 1303.5

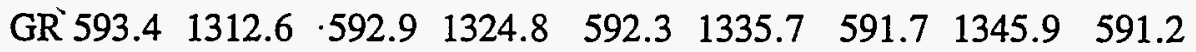
1358.1

$\begin{array}{lllllllll}\text { GR } 590.9 & 1368.7 & 590.3 & 1380.0 & 589.4 & 1389.9 & 588.8 & 1403.3 & 588.4\end{array}$ 1411.0

$\begin{array}{lllllllll}\text { GR } 588.2 & 1424.1 & 588.2 & 1432.2 & 587.4 & 1445.6 & 587.2 & 1454.0 & 586.9\end{array}$ 1466.1

$\begin{array}{lllllllll}\text { GR } 586.7 & 1476.4 & 586.6 & 1487.1 & 586.0 & 1497.7 & 585.7 & 1507.6 & 585.3\end{array}$ 1517.5

$\begin{array}{lllllllll}\text { GR } 586.6 & 1527.9 & 588.7 & 1538.3 & 589.8 & 1549.8 & 590.0 & 1557.8 & 591.5\end{array}$ 1572.0

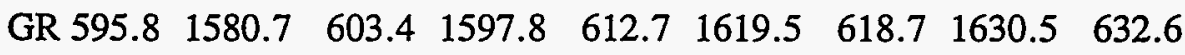
1662.2

GR 645.71709 .7

$\begin{array}{lllllllll}\mathrm{X} 1103.00 & 81 & 500.0 & 1606.3 & 1056 & 1056 & 1056 & .0000 & .00\end{array}$ $\begin{array}{lllllllll}\text { GR } 644.3 & 500.0 & 637.5 & 541.7 & 626.3 & 582.6 & 622.8 & 604.0 & 620.7\end{array}$ 627.0

$\begin{array}{lllllllll}\text { GR } 618.4 & 646.8 & 617.9 & 663.8 & 615.6 & 683.3 & 613.6 & 698.6 & 610.6\end{array}$ 715.7

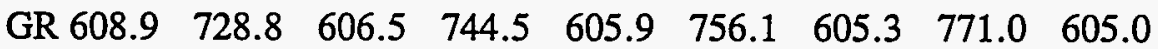
783.7

$\begin{array}{lllllllll}\text { GR } 604.9 & 797.3 & 604.5 & 810.8 & 604.2 & 824.7 & 604.1 & 837.0 & 603.9\end{array}$ 851.8

$\begin{array}{lllllllll}\text { GR } 603.6 & 862.9 & 603.5 & 874.6 & 603.3 & 889.0 & 603.0 & 902.0 & 602.6\end{array}$ 914.1

$\begin{array}{lllllllll}\text { GR } 602.7 & 928.7 & 602.4 & 938.9 & 601.9 & 952.5 & 601.6 & 964.6 & 601.3\end{array}$ 976.8

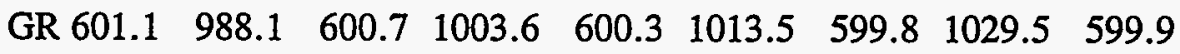
1037.0

$\begin{array}{lllllllll}\text { GR } 599.4 & 1052.4 & 599.1 & 1060.5 & 598.9 & 1073.7 & 598.6 & 1084.1 & 598.2\end{array}$ 1094.4

$\begin{array}{lllllllll}\text { GR } 598.0 & 1107.3 & 597.6 & 1115.3 & 597.2 & 1131.4 & 596.6 & 1146.5 & 596.4\end{array}$ 1153.7

$\begin{array}{lllllllll}\text { GR } 595.7 & 1169.3 & 595.2 & 1176.2 & 594.5 & 1191.4 & 594.1 & 1197.9 & 593.8\end{array}$ 1207.4

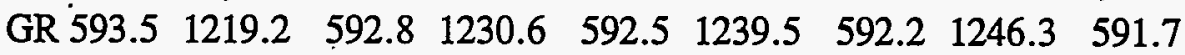
1261.4

$\begin{array}{lllllllll}\text { GR } 591.4 & 1272.8 & 591.1 & 1281.7 & 590.1 & 1288.4 & 591.4 & 1302.0 & 591.9\end{array}$ 1310.5

$\begin{array}{lllllllll}\text { GR } 590.0 & 1323.2 & 591.2 & 1331.3 & 589.9 & 1342.3 & 587.6 & 1351.9 & 589.3\end{array}$ 1362.2

$\begin{array}{lllllllll}\text { GR } 590.8 & 1372.7 & 590.0 & 1382.6 & 589.3 & 1394.5 & 589.5 & 1402.3 & 589.9\end{array}$ 1415.1

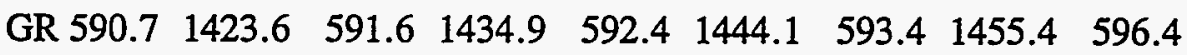
1465.0 
$\begin{array}{lllllllll}\text { GR } 601.1 & 1479.9 & 605.7 & 1490.3 & 615.2 & 1508.8 & 625.1 & 1524.4 & 637.2\end{array}$ 1561.3

GR $646.8 \quad 1606.3$

$\begin{array}{lllllllll}\mathrm{X} 1103.20 & 81 & 500.0 & 1601.2 & 1056 & 1056 & 1056 & .0000 & .00\end{array}$

$\begin{array}{lllllllll}\text { GR } 646.7 & 500.0 & 632.8 & 536.8 & 621.3 & 572.3 & 619.8 & 591.9 & 617.7\end{array}$

610.0

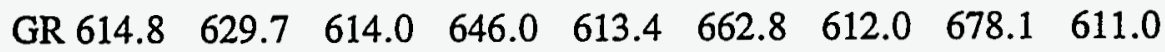
692.4

$\begin{array}{lllllllll}\text { GR } 609.3 & 710.2 & 608.5 & 725.0 & 608.3 & 739.7 & 608.0 & 754.5 & 607.6\end{array}$ 768.5

$\begin{array}{lllllllll}\text { GR } 607.1 & 781.9 & 606.8 & 796.5 & 606.7 & 813.9 & 606.5 & 825.7 & 606.0\end{array}$

841.1

$\begin{array}{lllllllll}\text { GR } 605.9 & 851.8 & 605.5 & 868.7 & 605.4 & 880.6 & 605.1 & 894.9 & 604.8\end{array}$

906.8

$\begin{array}{lllllllll}\text { GR } 604.4 & 921.1 & 604.0 & 933.1 & 603.6 & 945.7 & 603.1 & 960.6 & 602.4\end{array}$

976.6

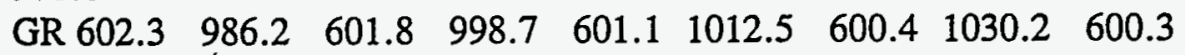
1037.0

$\begin{array}{lllllllll}\text { GR } 599.4 & 1053.7 & 599.0 & 1061.6 & 598.5 & 1075.4 & 598.0 & 1084.7 & 597.4\end{array}$ 1095.7

$\begin{array}{lllllllll}\text { GR 597.3 } & 1108.2 & 596.5 & 1120.3 & 596.3 & 1132.2 & 595.5 & 1148.8 & 595.5\end{array}$ 1154.6

$\begin{array}{lllllllll}\text { GR } 594.4 & 1170.2 & 594.0 & 1176.5 & 594.4 & 1190.1 & 594.2 & 1197.8 & 593.6\end{array}$ 1210.2

$\begin{array}{lllllllll}\text { GR } 593.6 & 1220.1 & 593.8 & 1229.9 & 593.6 & 1242.9 & 592.6 & 1251.1 & 593.4\end{array}$ 1264.4

$\begin{array}{lllllllll}\text { GR } 594.3 & 1272.4 & 593.3 & 1285.8 & 592.5 & 1293.2 & 593.5 & 1306.9 & 592.9\end{array}$ 1313.8

$\begin{array}{lllllllll}\text { GR } 591.7 & 1328.4 & 591.5 & 1335.7 & 590.6 & 1349.9 & 590.4 & 1356.9 & 591.5\end{array}$ 1370.0

$\begin{array}{lllllllll}\text { GR } 592.6 & 1377.8 & 592.7 & 1393.0 & 592.5 & 1403.0 & 593.6 & 1414.6 & 592.5\end{array}$ 1424.4

$\begin{array}{lllllllll}\text { GR } 591.8 & 1435.6 & 592.7 & 1449.9 & 594.1 & 1457.5 & 594.2 & 1467.4 & 595.2\end{array}$ 1478.1

$\begin{array}{lllllllll}\text { GR } 596.1 & 1482.6 & 603.8 & 1503.6 & 611.9 & 1522.8 & 618.7 & 1534.9 & 633.1\end{array}$ 1565.6

GR 645.41601 .2

$\begin{array}{lllllllll}\mathrm{X} 1103.39 & 81 & 500.0 & 1717.1 & 1003 & 1003 & 1003 & .0000 & .00\end{array}$ $\begin{array}{lllllllll}\text { GR } 657.2 & 500.0 & 636.9 & 539.8 & 621.1 & 575.7 & 615.0 & 596.2 & 612.5\end{array}$ 613.3

$\begin{array}{lllllllll}\text { GR } 609.2 & 630.8 & 608.3 & 645.6 & 607.2 & 660.9 & 606.0 & 676.6 & 604.9\end{array}$ 692.2

$\begin{array}{lllllllll}\text { GR } 603.8 & 707.7 & 602.5 & 723.2 & 601.4 & 736.7 & 600.5 & 749.9 & 599.7\end{array}$ 765.4

$\begin{array}{lllllllll}\text { GR } 599.3 & 780.1 & 599.1 & 793.0 & 598.8 & 805.8 & 598.5 & 820.2 & 598.1\end{array}$

836.6

$\begin{array}{lllllllll}\text { GR 598.0 } & 848.3 & 597.9 & 862.3 & 598.0 & 873.6 & 598.0 & 888.6 & 598.3\end{array}$ 
901.8

$\begin{array}{lllllllll}\text { GR } 598.4 & 914.5 & 598.6 & 928.3 & 599.1 & 942.1 & 599.2 & 955.8 & 599.4\end{array}$ 970.0

$\begin{array}{lllllllll}\text { GR } 599.6 & 984.4 & 599.7 & 997.0 & 599.7 & 1012.3 & 599.6 & 1023.9 & 599.8\end{array}$ 1039.7

$\begin{array}{lllllllll}\text { GR } 599.8 & 1056.3 & 599.9 & 1068.4 & 599.9 & 1083.2 & 599.1 & 1096.7 & 598.4\end{array}$ 1109.5

$\begin{array}{lllllllll}\text { GR } 598.1 & 1122.8 & 598.0 & 1135.9 & 596.6 & 1151.2 & 596.0 & 1166.6 & 595.4\end{array}$ 1176.0

$\begin{array}{lllllllll}\text { GR } 594.2 & 1188.9 & 593.7 & 1201.7 & 592.9 & 1212.8 & 592.2 & 1227.0 & 592.1\end{array}$ 1239.0

$\begin{array}{lllllllll}\text { GR } 592.5 & 1251.2 & 592.3 & 1263.2 & 592.2 & 1276.3 & 592.4 & 1290.4 & 592.7\end{array}$ 1302.4

$\begin{array}{lllllllll}\text { GR } 592.5 & 1316.0 & 592.7 & 1326.9 & 592.5 & 1337.7 & 592.1 & 1351.2 & 592.3\end{array}$ 1364.3

$\begin{array}{lllllllll}\text { GR } 593.2 & 1377.4 & 593.1 & 1390.7 & 593.0 & 1401.4 & 593.1 & 1412.2 & 593.7\end{array}$ 1428.0

$\begin{array}{lllllllll}\text { GR } 594.1 & 1444.8 & 594.0 & 1452.9 & 594.3 & 1466.0 & 594.8 & 1478.2 & 595.5\end{array}$ 1488.7

$\begin{array}{lllllllll}\text { GR } 596.9 & 1504.9 & 599.1 & 1521.1 & 600.0 & 1532.0 & 603.2 & 1548.3 & 610.9\end{array}$ 1561.8

$\begin{array}{lllllllll}\text { GR } 611.4 & 1579.1 & 622.0 & 1599.5 & 628.3 & 1627.4 & 629.1 & 1648.7 & 638.6\end{array}$ 1682.3

GR 644.01717 .1

$\begin{array}{lllllllll}\mathrm{X} 1103.57 & 81 & 500.0 & 1833.5 & 950 & 950 & 950 & .0000 & .00\end{array}$

$\begin{array}{lllllllll}\text { GR } 669.0 & 500.0 & 639.0 & 543.3 & 625.9 & 579.5 & 624.6 & 600.8 & 621.9\end{array}$

625.4

$\begin{array}{lllllllll}\text { GR } 618.1 & 646.3 & 613.6 & 667.5 & 609.7 & 687.8 & 606.8 & 704.4 & 603.8\end{array}$ 724.7

$\begin{array}{lllllllll}\text { GR } 602.2 & 737.0 & 600.4 & 756.9 & 599.3 & .769 .8 & 598.9 & 788.9 & 597.1\end{array}$ 800.8

$\begin{array}{lllllllll}\text { GR } 595.4 & 816.1 & 593.7 & 829.3 & 593.2 & 842.9 & 594.4 & 859.0 & 595.8\end{array}$ 875.2

$\begin{array}{lllllllll}\text { GR } 597.4 & 888.8 & 599.3 & 907.3 & 600.2 & 920.3 & 600.9 & 938.4 & 600.8\end{array}$ 951.9

$\begin{array}{lllllllll}\text { GR } 600.5 & 970.4 & 600.3 & 983.4 & 599.8 & 1002.6 & 599.6 & 1015.9 & 598.8\end{array}$ 1033.5

$\begin{array}{lllllllll}\text { GR } 598.5 & 1046.6 & 598.0 & 1065.0 & 597.5 & 1076.9 & 597.4 & 1097.8 & 597.1\end{array}$ 1106.5

$\begin{array}{lllllllll}\text { GR } 596.9 & 1124.2 & 596.7 & 1136.8 & 596.4 & 1155.5 & 596.1 & 1168.0 & 595.8\end{array}$ 1187.4

$\begin{array}{lllllllll}\text { GR } 595.7 & 1198.2 & 595.6 & 1212.9 & 595.3 & 1228.0 & 594.7 & 1245.1 & 594.5\end{array}$ 1256.3

$\begin{array}{lllllllll}\text { GR } 593.9 & 1271.6 & 593.8 & 1286.0 & 593.1 & 1303.1 & 592.8 & 1314.7 & 592.6\end{array}$ 1329.4

$\begin{array}{lllllllll}\text { GR } 592.4 & 1342.7 & 592.6 & 1356.7 & 593.1 & 1373.3 & 593.8 & 1388.7 & 594.2\end{array}$ 1401.5 
$\begin{array}{lllllllll}\text { GR } 594.5 & 1419.3 & 595.1 & 1430.8 & 595.9 & 1446.3 & 596.6 & 1459.6 & 597.2\end{array}$ 1472.5

$\begin{array}{lllllllll}\text { GR } 597.0 & 1491.6 & 596.8 & 1504.8 & 596.0 & 1522.2 & 595.9 & 1536.5 & 596.9\end{array}$ 1550.9

$\begin{array}{lllllllll}\text { GR } 597.8 & 1569.6 & 596.2 & 1581.6 & 596.7 & 1600.6 & 597.7 & 1612.1 & 598.0\end{array}$ 1627.3

$\begin{array}{lllllllll}\text { GR } 598.3 & 1641.2 & 598.4 & 1654.2 & 600.0 & 1672.9 & 600.8 & 1685.4 & 607.3\end{array}$ 1706.6

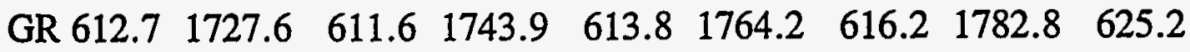
1802.4

GR 643.81833 .5

$\begin{array}{lllllllll}\mathrm{X} 1103.73 & 81 & 500.0 & 2521.8 & 845 & 845 & 845 & .0000 & .00\end{array}$ $\begin{array}{lllllllll}\text { GR } 646.6 & 500.0 & 632.4 & 547.9 & 617.2 & 601.8 & 611.5 & 621.6 & 609.4\end{array}$ 649.7

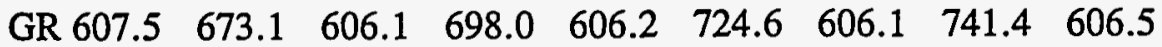

766.6

$\begin{array}{lllllllll}\text { GR } 606.6 & 786.2 & 606.5 & 806.9 & 605.6 & 828.1 & 605.6 & 850.1 & 606.2\end{array} \cdot$ 871.0

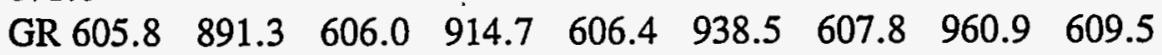
986.3

$\begin{array}{lllllllll}\text { GR } 611.2 & 1007.0 & 613.3 & 1032.9 & 615.2 & 1059.0 & 616.1 & 1085.5 & 614.3\end{array}$ 1111.5

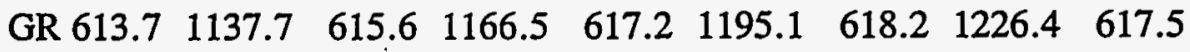
1257.6

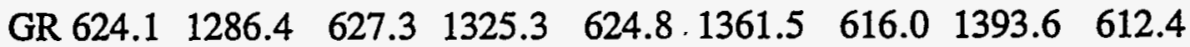
1422.6

$\begin{array}{lllllllll}\text { GR } 609.8 & 1446.7 & 605.7 & 1468.1 & 601.8 & 1489.9 & 600.3 & 1508.9 & 599.7\end{array}$ 1526.3

$\begin{array}{lllllllll}\text { GR } 598.7 & 1547.6 & 597.5 & 1567.8 & 596.2 & 1584.4 & 595.1 & 1603.0 & 595.8\end{array}$ 1620.5

$\begin{array}{lllllllll}\text { GR } 594.3 & 1639.9 & 595.9 & 1656.4 & 597.8 & 1673.4 & 598.0 & 1692.7 & 598.4\end{array}$ 1710.5

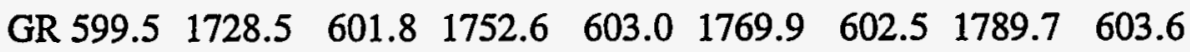
1811.2

$\begin{array}{lllllllll}\text { GR } 605.0 & 1834.1 & 606.9 & 1852.9 & 608.2 & 1872.0 & 608.5 & 1898.1 & 608.2\end{array}$ 1924.8

$\begin{array}{lllllllll}\text { GR } 607.6 & 1943.2 & 607.3 & 1966.8 & 607.3 & 1988.1 & 606.7 & 2008.8 & 607.7\end{array}$ 2035.1

GR 609.2 $2061.6 \quad 611.0 \quad 2080.4 \quad 612.6 \quad 2109.6 \quad 613.6 \quad 2132.4 \quad 615.0$ 2162.7

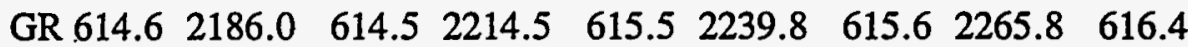
2292.8

$\begin{array}{lllllllll}\text { GR } 618.8 & 2319.9 & 620.5 & 2354.6 & 620.6 & 2394.7 & 623.0 & 2420.7 & 628.3\end{array}$

2467.1

GR 646.62521 .8

$\begin{array}{lllllllll}\mathrm{X} 1103.85 & 81 & 500.0 & 2862.6 & 634 & 634 & 634 & .0000 & .00\end{array}$

$\begin{array}{lllllllll}\text { GR } 666.3 & 500.0 & 632.4 & 558.0 & 619.5 & 600.4 & 616.2 & 631.3 & 615.7\end{array}$ 
659.0

$\begin{array}{lllllllll}\text { GR } 618.3 & 688.8 & 620.3 & 717.0 & 620.3 & 750.3 & 618.7 & 781.2 & 617.6\end{array}$ 811.4

$\begin{array}{lllllllll}\text { GR } 614.6 & 838.7 & 613.3 & 867.3 & 613.7 & 895.6 & 615.5 & 922.3 & 615.8\end{array}$ 949.8

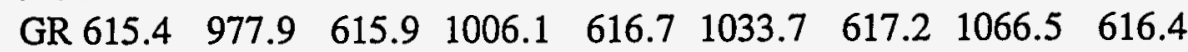
$\cdot 1099.5$

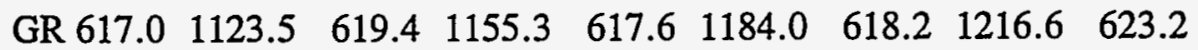
1246.3

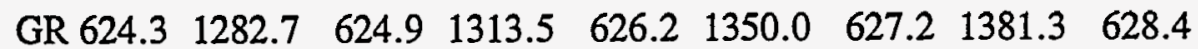
1416.9

$\begin{array}{lllllllll}\text { GR } 629.2 & 1455.1 & 630.5 & 1497.1 & 630.7 & 1533.0 & 630.9 & 1570.3 & 630.6\end{array}$ 1609.9

$\begin{array}{llllllll}\text { GR } 629.9 & 1652.5 & 630.1 & 1689.6 & 630.5 \cdot 1723.2 & 623.6 & 1761.7 & 612.1\end{array}$ 1789.2

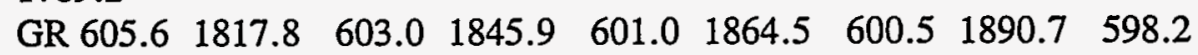
1908.3

$\begin{array}{lllllllll}\text { GR } 597.0 & 1934.3 & 596.4 & 1952.1 & 595.6 & 1972.8 & 595.0 & 1992.6 & 595.1\end{array}$ 2011.3

$\begin{array}{lllllllll}\text { GR } 594.9 & 2033.2 & 593.9 & 2055.4 & 594.4 & 2075.2 & 595.0 & 2099.3 & 596.8\end{array}$ 2118.0

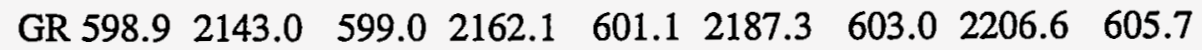
2231.0

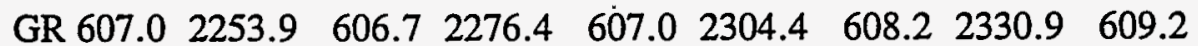
2352.3

$\begin{array}{lllllllll}\text { GR } 610.2 & 2380.2 & 611.8 & 2405.8 & 613.4 & 2435.5 & 614.3 & 2460.0 & 615.2\end{array}$ 2491.6

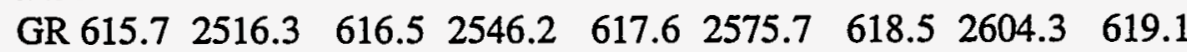
2635.5

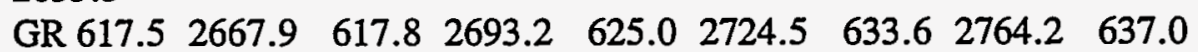
2810.6

GR 649.62862 .6

$\begin{array}{lllllllll}\mathrm{X} 1104.05 & 81 & 500.0 & 2599.0 & 1056 & 1056 & 1056 & .0000 & .00\end{array}$

$\begin{array}{lllllllll}\text { GR } 700.0 & 500.0 & 647.0 & 560.2 & 624.2 & 596.6 & 621.6 & 622.5 & 622.7\end{array}$

652.5

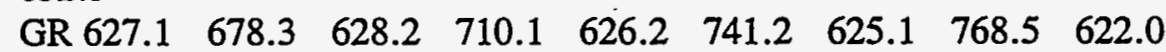

794.3

$\begin{array}{lllllllll}\text { GR } 618.7 & 819.9 & 616.7 & 847.4 & 615.0 & 874.3 & 613.5 & 895.6 & 612.2\end{array}$ 923.0

$\begin{array}{lllllllll}\text { GR } 612.5 & 951.3 & 613.1 & 972.9 & 613.8 & 995.2 & 616.3 & 1022.3 & 619.3\end{array}$ 1053.1

$\begin{array}{lllllllll}\text { GR } 619.3 & 1075.0 & 621.2 & 1103.8 & 622.7 & 1127.7 & 623.7 & 1156.7 & 621.9\end{array}$ 1182.7

$\begin{array}{lllllllll}\text { GR } 625.2 & 1208.5 & 626.0 & 1239.6 & 626.6 & 1271.6 & 627.4 & 1298.0 & 627.8\end{array}$ 1323.4

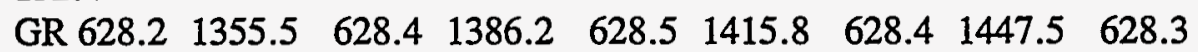
1473.8 


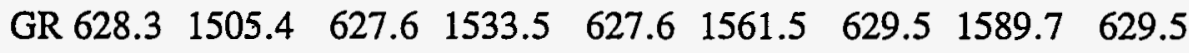
1618.3

$\begin{array}{lllllllll}\text { GR } 629.3 & 1648.4 & 628.7 & 1676.4 & 627.4 & 1707.7 & 626.3 & 1735.4 & 624.6\end{array}$ 1766.0

$\begin{array}{lllllllll}\text { GR } 623.5 & 1791.1 & 621.6 & 1821.2 & 619.2 & 1846.4 & 616.5 & 1874.9 & 614.1\end{array}$ 1896.8

$\begin{array}{lllllllll}\text { GR } 610.5 & 1925.2 & 606.8 & 1951.3 & 604.8 & 1970.0 & 602.6 & 1995.8 & 602.0\end{array}$ 2015.8

$\begin{array}{lllllllll}\text { GR } 601.0 & 2039.5 & 600.3 & 2058.1 & 600.1 & 2082.7 & 599.8 & 2102.2 & 599.5\end{array}$ 2121.9

$\begin{array}{lllllllll}\text { GR } 599.3 & 2144.8 & 599.1 & 2166.4 & 599.1 & 2185.8 & 599.1 & 2204.6 & 598.9\end{array}$ 2229.2

$\begin{array}{lllllllll}\text { GR } 598.7 & 2248.6 & 598.0 & 2271.4 & 597.4 & 2294.1 & 597.6 & 2313.1 & 598.0\end{array}$ 2338.0

$\begin{array}{lllllllll}\text { GR } 598.8 & 2357.3 & 599.3 & 2377.0 & 600.8 & 2398.8 & 600.3 & 2420.7 & 600.7\end{array}$ 2443.1

$\begin{array}{lllllllll}\text { GR } 603.3 & 2470.2 & 605.5 & 2486.9 & 610.9 & 2515.7 & 622.4 & 2535.5 & 636.1\end{array}$ 2562.2

GR $644.2 \div 2599.0$

$\begin{array}{lllllllll}\mathrm{X} 1104.26 & 81 & 500.0 & 2314.4 & 1109 & 1109 & 1109 & .0000 & .00\end{array}$ $\begin{array}{lllllllll}\text { GR } 766.4 & 500.0 & 717.0 & 581.5 & 664.4 & 634.4 & 638.9 & 665.5 & 632.2\end{array}$ 686.6

$\begin{array}{lllllllll}\text { GR } 624.6 & 711.0 & 627.3 & 734.7 & 631.6 & 753.2 & 633.2 & 780.7 & 633.1\end{array}$ 827.1

$\begin{array}{lllllllll}\text { GR } 633.0 & 831.6 & 630.9 & 856.1 & 626.0 & 878.1 & 621.9 & 903.5 & 619.7\end{array}$ 922.0

$\begin{array}{lllllllll}\text { GR } 618.2 & 945.6 & 617.6 & 966.2 & 616.8 & 987.3 & 615.8 & 1008.4 & 614.9\end{array}$ 1030.8

$\begin{array}{lllllllll}\text { GR } 613.9 & 1052.7 & 613.3 & 1073.3 & 612.3 & 1094.6 & 611.6 & 1116.8 & 611.4\end{array}$ 1136.6

$\begin{array}{lllllllll}\text { GR } 612.0 & 1161.5 & 611.6 & 1178.9 & 613.4 & 1199.8 & 618.0 & 1222.4 & 619.7\end{array}$ 1244.0

$\begin{array}{lllllllll}\text { GR } 621.5 & 1265.7 & 622.2 & 1291.6 & 622.7 & 1309.4 & 623.0 & 1333.8 & 623.3\end{array}$ 1356.9

$\begin{array}{lllllllll}\text { GR } 623.5 & 1378.9 & 623.6 & 1399.7 & 623.5 & 1421.5 & 623.4 & 1445.9 & 622.9\end{array}$ 1465.5

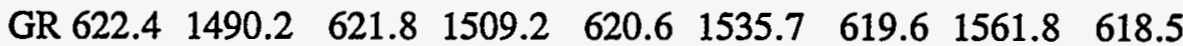
1579.6

$\begin{array}{lllllllll}\text { GR } 617.2 & 1600.9 & 615.5 & 1622.5 & 614.3 & 1642.9 & 612.5 & 1665.6 & 610.4\end{array}$ 1686.7

$\begin{array}{lllllllll}\text { GR } 609.0 & 1707.8 & 607.5 & 1729.3 & 606.8 & 1748.7 & 605.8 & 1767.7 & 604.9\end{array}$ 1789.8

$\begin{array}{lllllllll}\text { GR } 604.1 & 1807.2 & 602.7 & 1828.8 & 601.8 & 1851.2 & 601.1 & 1868.6 & 600.6\end{array}$ 1885.0

$\begin{array}{lllllllll}\text { GR } 599.9 & 1907.5 & 599.0 & 1929.3 & 598.5 & 1945.8 & 598.1 & 1966.5 & 597.8\end{array}$ 1985.7

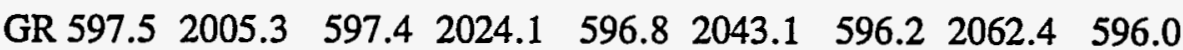


2082.4

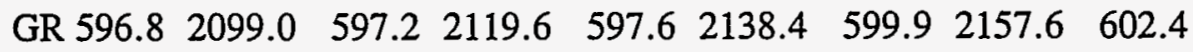
2176.9

$\begin{array}{lllllllll}\text { GR } 604.9 & 2194.8 & 613.1 & 2217.8 & 623.7 & 2241.3 & 634.5 & 2263.6 & 641.3\end{array}$ 2289.3

GR 647.22314 .4

$\begin{array}{lllllllll}\mathrm{X} 1104.45 & 81 & 500.0 & 1703.8 & 1003 & 1003 & 1003 & .0000 & .00\end{array}$

$\begin{array}{lllllllll}\text { GR } 646.0 & 500.0 & 641.6 & 583.1 & 629.1 & 621.7 & 619.8 & 640.3 & 614.0\end{array}$

661.6

$\begin{array}{lllllllll}\text { GR } 609.9 & 676.1 & 608.0 & 691.3 & 606.3 & 706.5 & 604.7 & 718.4 & 602.0\end{array}$

732.3

$\begin{array}{lllllllll}\text { GR } 601.2 & 743.0 & 600.1 & 753.2 & 598.9 & 766.1 & 598.5 & 774.1 & 598.4\end{array}$ 788.9

$\begin{array}{lllllllll}\text { GR } 598.9 & 801.1 & 600.9 & 813.1 & 603.8 & 827.1 & 606.8 & 839.5 & 610.3\end{array}$ 855.3

$\begin{array}{lllllllll}\text { GR } 613.1 & 869.2 & 615.2 & 885.9 & 614.9 & 901.3 & 614.8 & 917.7 & 615.3\end{array}$ 933.5

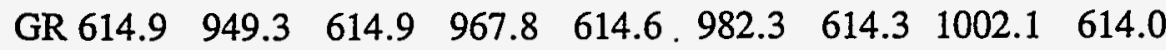
1015.8

$\begin{array}{lllllllll}\text { GR.613.5 } & 1033.1 & 612.9 & 1050.7 & 611.9 & 1066.5 & 610.9 & 1085.2 & 610.5\end{array}$ 1096.0

$\begin{array}{lllllllll}\text { GR } 609.4 & 1112.7 & 608.6 & 1125.1 & 608.0 & 1138.3 & 606.7 & 1152.5 & 606.0\end{array}$ 1165.7

$\begin{array}{lllllllll}\text { GR } 605.3 & 1179.3 & 604.5 & 1191.8 & 604.0 & 1205.1 & 603.5 & 1216.3 & 603.0\end{array}$ 1231.4

$\begin{array}{llllllll}\text { GR } 602.8 & 1241.2 & 602.3 & 1256.0 & 601.4 .1271 .2 & 601.3 & 1280.7 & 601.1\end{array}$ 1293.1

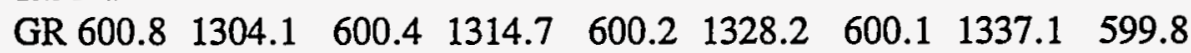
1351.6

$\begin{array}{lllllllll}\text { GR } 599.5 & 1359.7 & 599.1 & 1375.6 & 598.7 & 1391.6 & 598.5 & 1399.2 & 598.1\end{array}$ 1411.0

$\begin{array}{lllllllll}\text { GR } 598.2 & 1421.3 & 597.7 & 1430.8 & 597.4 & 1444.9 & 597.1 & 1460.8 & 597.0\end{array}$ 1466.7

$\begin{array}{lllllllll}\text { GR } 596.6 & 1480.6 & 596.3 & 1488.2 & 596.0 & 1502.2 & 596.0 & 1511.1 & 595.7\end{array}$ 1523.4

$\begin{array}{lllllllll}\text { GR } 595.4 & 1533.0 & 595.1 & 1544.6 & 595.0 & 1554.9 & 595.4 & 1565.5 & 598.4\end{array}$ 1576.1

$\begin{array}{lllllllll}\text { GR } 600.8 & 1586.7 & 604.7 & 1601.2 & 611.1 & 1619.1 & 618.4 & 1630.2 & 635.2\end{array}$ 1660.9

GR 645.21703 .8

$\begin{array}{lllllllll}\mathrm{X} 1104.67 & 81 & 500.0 & 1845.3 & 1162 & 1162 & 1162 & .0000 & .00\end{array}$

$\begin{array}{lllllllll}\text { GR } 643.0 & 500.0 & 633.3 & 544.3 & 625.9 & 584.5 & 622.1 & 608.9 & 620.6\end{array}$

630.9

$\begin{array}{lllllllll}\text { GR } 617.9 & 654.8 & 617.3 & 672.8 & 615.8 & 690.5 & 612.4 & 710.9 & 611.8\end{array}$ 731.3

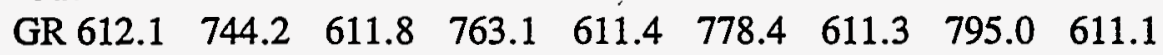
811.2 
$\begin{array}{lllllllll}\text { GR } 610.5 & 827.2 & 608.4 & 845.8 & 607.4 & 859.1 & 609.4 & 877.1 & 611.5\end{array}$ 890.5

$\begin{array}{lllllllll}\text { GR } 615.4 & 911.3 & 618.8 & 932.3 & 622.1 & 953.1 & 620.9 & 978.5 & 618.0\end{array}$ 997.2

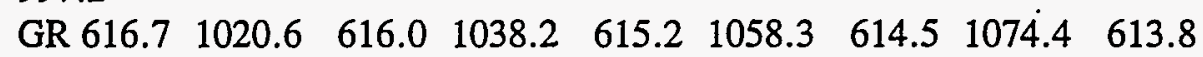
1090.5

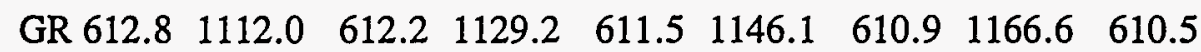
1179.1

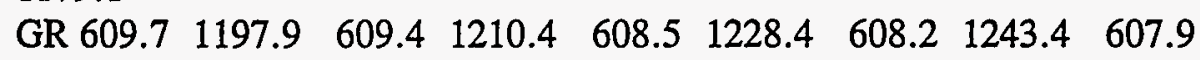
1255.2

$\begin{array}{lllllllll}\text { GR } 607.5 & 1273.1 & 607.0 & 1287.3 & 606.1 & 1304.3 & 605.4 & 1320.7 & 604.6\end{array}$ 1334.0

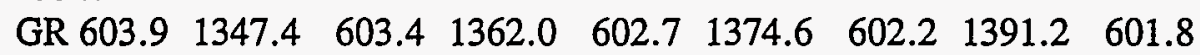
1402.5

$\begin{array}{lllllllll}\text { GR } 601.2 & 1416.7 & 600.7 & 1430.8 & 600.2 & 1444.6 & 599.9 & 1457.7 & 599.8\end{array}$ 1469.7

$\begin{array}{lllllllll}\text { GR } 599.5 & 1481.4 & 599.4 & 1495.9 & 599.2 & 1508.6 & 598.8 & 1522.1 & 598.6\end{array}$ 1536.5

$\begin{array}{lllllllll}\text { GR } 598.5 & 1546.7 & 598.4 & 1557.1 & 597.9 & 1572.6 & 598.0 & 1585.7 & 597.5\end{array}$ 15.99 .1

$\begin{array}{lllllllll}\text { GR } 597.1 & 1614.0 & 597.0 & 1622.6 & 596.5 & 1636.0 & 596.3 & 1647.0 & 596.2\end{array}$ 1657.1

$\begin{array}{lllllllll}\text { GR } 595.8 & 1671.3 & 595.6 & 1679.0 & 594.7 & 1696.2 & 594.3 & 1712.3 & 594.1\end{array}$ 1720.1

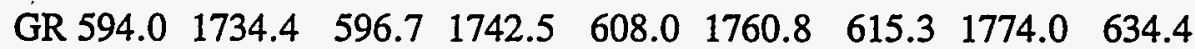
1810.5

GR 645.41845 .3

$\begin{array}{lllllllll}\mathrm{X} 1104.85 & 81 & 500.0 & 2173.0 & 950 & 950 & 950 & .0000 & .00\end{array}$

$\begin{array}{lllllllll}\text { GR } 660.4 & 500.0 & 640.2 & 547.3 & 628.3 & 593.6 & 623.3 & 618.0 & 617.0\end{array}$

642.3

$\begin{array}{lllllllll}\text { GR } 613.2 & 659.4 & 612.1 & 681.9 & 613.0 & 701.6 & 613.6 & 722.2 & 614.0\end{array}$ 746.9

$\begin{array}{lllllllll}\text { GR } 614.2 & 762.6 & 614.9 & 786.6 & 615.1 & 803.3 & 615.9 & 823.5 & 616.2\end{array}$ 845.7

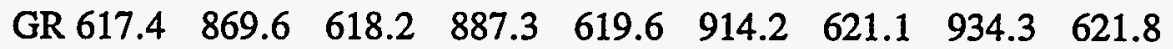
942.6

$\begin{array}{lllllllll}\text { GR } 631.7 & 987.6 & 635.8 & 1017.0 & 635.1 & 1055.1 & 634.0 & 1078.9 & 630.5\end{array}$ 1121.0

$\begin{array}{lllllllll}\text { GR } 627.6 & 1145.2 & 626.0 & 1175.5 & 623.8 & 1203.4 & 621.7 & 1226.6 & 619.3\end{array}$ 1250.2

$\begin{array}{lllllllll}\text { GR } 618.2 & 1270.8 & 616.3 & 1294.6 & 614.2 & 1312.4 & 612.9 & 1332.7 & 611.4\end{array}$ 1352.9

$\begin{array}{lllllllll}\text { GR } 610.8 & 1372.1 & 609.7 & 1390.3 & 609.2 & 1410.1 & 608.6 & 1429.0 & 608.3\end{array}$ 1447.1

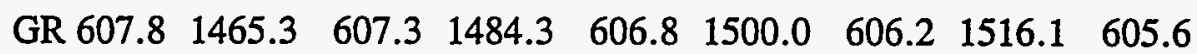
1535.8

$\begin{array}{lllllllll}\text { GR } 604.7 & 1558.5 & 604.6 & 1570.8 & 604.3 & 1588.5 & 603.7 & 1605.6 & 603.4\end{array}$ 
1625.7

$\begin{array}{lllllllll}\text { GR } 603.0 & 1638.4 & 602.6 & 1656.8 & 602.3 & 1672.4 & 602.0 & 1688.5 & 601.9\end{array}$ 1705.4

$\begin{array}{lllllllll}\text { GR } 601.9 & 1722.1 & 601.8 & 1736.3 & 602.0 & 1756.1 & 602.3 & 1769.8 & 602.3\end{array}$ 1789.0

$\begin{array}{lllllllll}\text { GR } 602.2 & 1802.0 & 602.5 & 1819.7 & 602.1 & 1836.2 & 602.1 & 1852.6 & 602.2\end{array}$ 1869.0

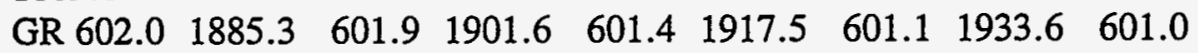
1950.0

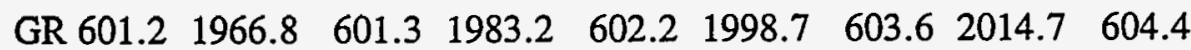
2033.4

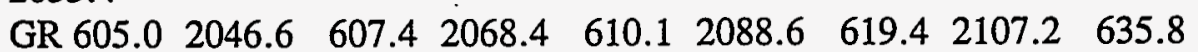
2140.1

GR 644.92173 .0

$\begin{array}{lllllllll}\mathrm{X} 1105.00 & 81 & 500.0 & 2333.9 & 792 & 792 & 792 & .0000 & .00\end{array}$

$\begin{array}{lllllllll}\text { GR } 659.5 & 500.0 & 634.8 & 540.6 & 619.6 & 580.4 & 615.0 & 603.1 & 611.6\end{array}$

625.8

$\begin{array}{lllllllll}\text { GR } 611.3 & 643.9 & 611.6 & 664.9 & 611.9 & 685.4 & 613.2 & 708.9 & 617.2\end{array}$

732.3

$\begin{array}{lllllllll}\text { GR } 624.7 & 756.5 & 630.0 & 785.3 & 629.0 & 815.8 & 630.1 & 851.8 & 630.0\end{array}$

879.4

$\begin{array}{lllllllll}\text { GR } 630.1 & 911.9 & 628.5 & 942.8 & 627.6 & 973.3 & 626.4 & 1000.3 & 625.3\end{array}$

1029.6

$\begin{array}{lllllllll}\text { GR } 624.2 & 1055.1 & 623.7 & 1080.3 & 622.3 & 1111.4 & 621.1 & 1137.5 & 619.4\end{array}$

1159.9

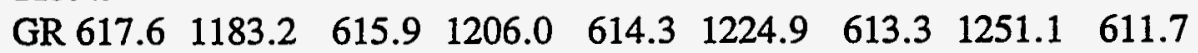
1272.9

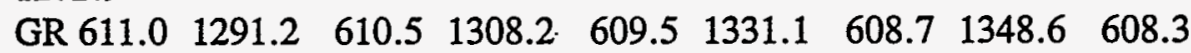
1370.9

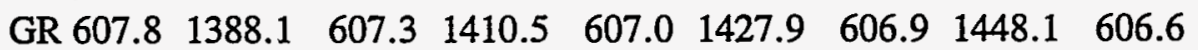
1469.4

$\begin{array}{lllllllll}\text { GR } 606.2 & 1486.5 & 605.5 & 1504.9 & 605.3 & 1523.4 & 605.1 & 1546.9 & 604.5\end{array}$ 1560.8

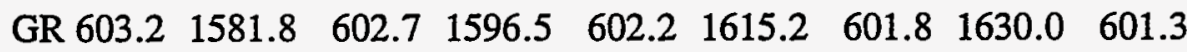
1648.5

$\begin{array}{lllllllll}\text { GR } 601.4 & 1666.2 & 601.5 & 1685.4 & 601.8 & 1698.9 & 601.9 & 1718.0 & 601.7\end{array}$ 1734.1

$\begin{array}{lllllllll}\text { GR } 602.1 & 1750.1 & 602.3 & 1771.1 & 602.2 & 1790.1 & 602.2 & 1805.8 & 602.2\end{array}$ 1821.7

$\begin{array}{lllllllll}\text { GR } 602.7 & 1841.4 & 603.5 & 1861.9 & 603.9 & 1876.0 & 605.4 & 1896.8 & 606.9\end{array}$ 1913.0

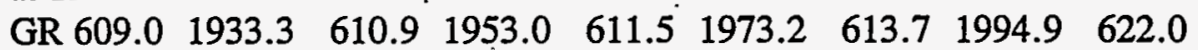
2023.9

$\begin{array}{lllllllll}\text { GR } 625.1 & 2043.5 & 629.6 & 2076.6 & 631.3 & 2103.9 & 630.3 & 2134.1 & 627.7\end{array}$ 2169.1

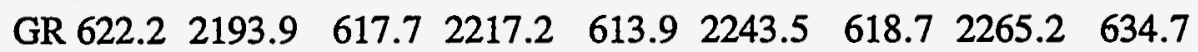
2297.5 
GR 644.62333 .9

$\begin{array}{lllllllll}\mathrm{X} 1105.16 & 81 & 500.0 & 2324.1 & 845 & 845 & 845 & .0000 & .00\end{array}$ $\begin{array}{lllllllll}\text { GR } 650.4 & 500.0 & 631.3 & 542.9 & 622.1 & 586.3 & 618.3 & 610.2 & 616.5\end{array}$ 636.1

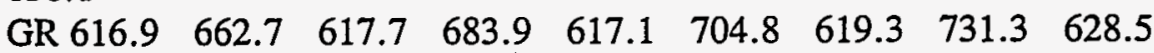
762.9

$\begin{array}{lllllllll}\text { GR } 628.2 & 794.7 & 627.4 & 825.5 & 626.1 & 858.3 & 622.7 & 888.8 & 619.8\end{array}$ 914.5

$\begin{array}{llllllll}\text { GR } 617.9 & 941.2 & 616.8 & 964.0 & 615.4 & 988.9 & 615.1 & 1009.6 \cdot 613.8\end{array}$ 1030.6

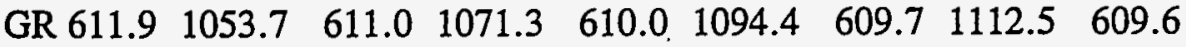
1133.0

$\begin{array}{lllllllll}\text { GR } 609.3 & 1153.5 & 609.4 & 1172.4 & 609.7 & 1196.7 & 609.6 & 1213.6 & 609.3\end{array}$ 1235.8

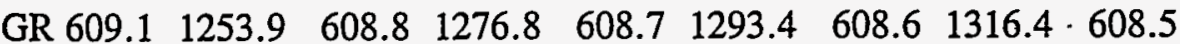
1332.5

$\begin{array}{lllllllll}\text { GR } 608.2 & 1353.0 & 607.8 & 1368.8 & 607.4 & 1389.8 & 607.4 & 1408.5 & 606.7\end{array}$ 1430.5

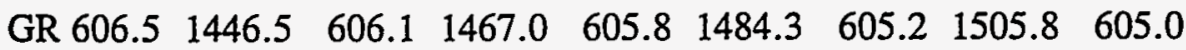
1518.5

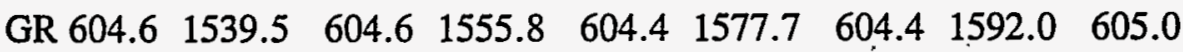
1612.3

$\begin{array}{lllllllll}\text { GR } 605.2 & 1626.6 & 605.5 & 1645.0 & 606.3 & 1663.1 & 606.7 & 1681.3 & 607.7\end{array}$ 1701.8

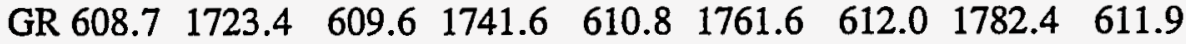
1804.3

$\begin{array}{lllllllll}\text { GR } 611.8 & 1823.1 & 611.7 & 1841.7 & 612.1 & 1864.0 & 610.9 & 1880.3 & 616.3\end{array}$ 1906.8

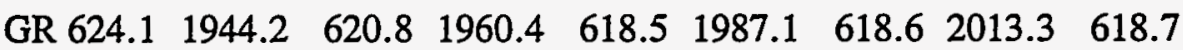
2034.6

$\begin{array}{lllllllll}\text { GR } 618.5 & 2060.5 & 618.1 & 2082.9 & 617.0 & 2111.1 & 616.1 & 2137.2 & 615.6\end{array}$ 2157.5

$\begin{array}{lllllllll}\text { GR } 614.4 & 2179.4 & 614.0 & 2201.0 & 614.3 & 2226.0 & 619.3 & 2247.9 & 631.1\end{array}$ 2283.8

GR 644.82324 .1

$\begin{array}{lllllllll}\mathrm{X} 1105.38 & 81 & 500.0 & 1703.3 & 1162 & 1162 & 1162 & .0000 & .00\end{array}$ $\begin{array}{lllllllll}\text { GR } 648.8 & 500.0 & 633.4 & 535.9 & 625.5 & 567.7 & 620.5 & 586.8 & 621.0\end{array}$ 604.5

$\begin{array}{lllllllll}\text { GR } 619.6 & 623.3 & 618.0 & 640.6 & 616.7 & 656.9 & 616.2 & 673.8 & 616.3\end{array}$ 690.9

$\begin{array}{lllllllll}\text { GR } 615.6 & 707.5 & 615.3 & 724.1 & 616.6 & 738.2 & 617.4 & 752.8 & 614.9\end{array}$ 770.2

$\begin{array}{lllllllll}\text { GR } 611.8 & 782.5 & 609.5 & 799.8 & 608.6 & 811.6 & 607.9 & 825.6 & 606.8\end{array}$ 840.4

$\begin{array}{lllllllll}\text { GR } 606.6 & 852.3 & 606.2 & 868.5 & 606.1 & 878.7 & 605.8 & 891.8 & 606.3\end{array}$ 904.7

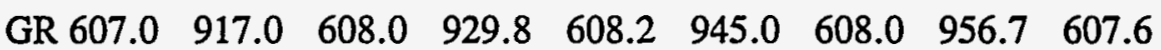


969.3

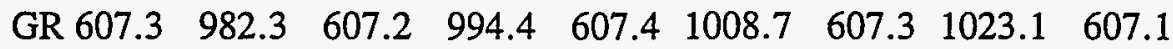
1035.3

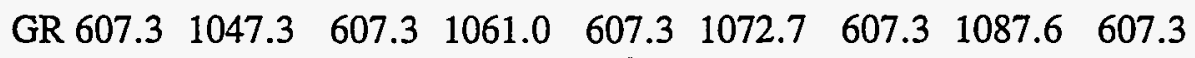
1101.6

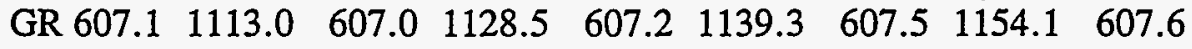
1164.9

$\begin{array}{lllllllll}\text { GR } 607.7 & 1177.2 & 607.8 & 1192.6 & 607.7 & 1208.1 & 607.7 & 1219.6 & 607.8\end{array}$ 1232.0

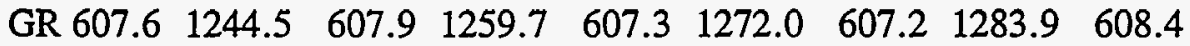
1298.8

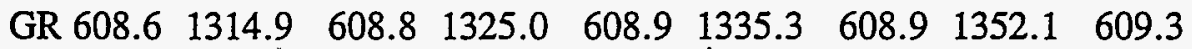
1366.0

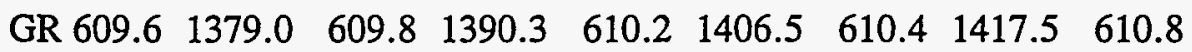
1434.0

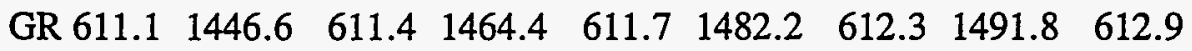
1507.4

$\begin{array}{lllllllll}\text { GR } 613.0 & 1521.0 & 613.3 & 1538.0 & 614.0 & 1553.3 & 614.6 & 1568.8 & 615.5\end{array}$ 1583.9

$\begin{array}{lllllllll}\text { GR } 615.8 & 1599.3 & 615.9 & 1614.4 & 616.1 & 1631.6 & 616.5 & 1647.6 & 629.3\end{array}$ 1666.9

GR 644.31703 .3

$\begin{array}{lllllllll}\mathrm{X} 1105.67 & 81 & 500.0 & 1814.0 & 1531 & 1531 & 1531 & .0000 & .00\end{array}$ $\begin{array}{lllllllll}\text { GR } 645.1 & 500.0 & 637.0 & 561.3 & 628.2 & 595.5 & 625.7 & 618.1 & 621.8\end{array}$ 640.3

$\begin{array}{lllllllll}\text { GR } 619.7 & 659.7 & 617.7 & 680.1 & 616.0 & 695.3 & 616.0 & 714.0 & 616.4\end{array}$ 726.5

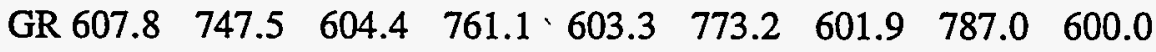
799.0

$\begin{array}{lllllllll}\text { GR } 598.9 & 812.6 & 597.7 & 821.2 & 596.9 & 832.6 & 597.1 & 843.5 & 597.5\end{array}$

854.0

$\begin{array}{lllllllll}\text { GR } 597.2 & 866.4 & 597.1 & 873.8 & 597.6 & 889.2 & 598.0 & 899.6 & 598.2\end{array}$

911.6

$\begin{array}{lllllllll}\text { GR } 598.4 & 918.1 & 599.9 & 934.4 & 600.6 & 943.8 & 601.3 & 958.4 & 601.9\end{array}$ 970.1

$\begin{array}{lllllllll}\text { GR } 602.4 & 982.2 & 602.7 & 995.7 & 603.1 & 1007.1 & 603.5 & 1021.5 & 604.4\end{array}$ 1033.5

$\begin{array}{lllllllll}\text { GR } 604.8 & 1047.8 & 605.3 & 1059.0 & 605.6 & 1069.9 & 606.1 & 1086.2 & 606.7\end{array}$ 1101.5

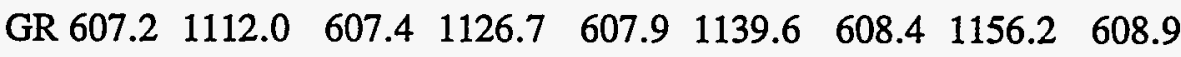
1168.2

$\begin{array}{lllllllll}\text { GR } 609.4 & 1186.8 & 609.5 & 1198.4 & 610.0 & 1211.7 & 610.5 & 1227.1 & 610.8\end{array}$ 1243.7

$\begin{array}{lllllllll}\text { GR } 611.3 & 1258.3 & 611.7 & 1276.7 & 612.0 & 1288.7 & 612.3 & 1308.4 & 612.8\end{array}$ 1319.2

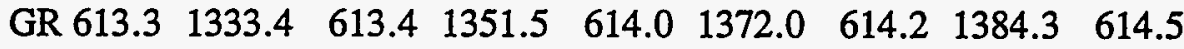
1402.7 


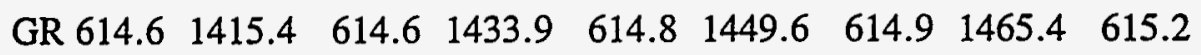
1483.6

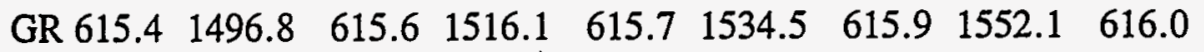
1570.9

$\begin{array}{lllllllll}\text { GR } 615.8 & 1584.1 & 615.6 & 1603.5 & 615.3 & 1619.4 & 615.6 & 1634.9 & 615.7\end{array}$ 1652.9

$\begin{array}{lllllllll}\text { GR } 616.0 & 1671.6 & 618.6 & 1689.7 & 621.5 & 1711.1 & 627.7 & 1728.9 & 634.6\end{array}$ 1766.4

GR 645.71814 .0

$\begin{array}{lllllllll}\mathrm{X} 1106.00 & 81 & 500.0 & 1675.0 & 1742 & 1742 & 1742 & .0000 & .00\end{array}$

$\begin{array}{lllllllll}\text { GR 649.1 } & 500.0 & 632.1 & 530.1 & 619.4 & 558.2 & 615.2 & 575.1 & 613.7\end{array}$

591.2

$\begin{array}{lllllllll}\text { GR } 610.4 & 613.3 & 610.0 & 620.3 & 608.2 & 634.0 & 608.9 & 645.6 & 610.3\end{array}$ 658.0

$\begin{array}{lllllllll}\text { GR } 612.0 & 674.2 & 612.5 & 688.5 & 612.3 & 702.9 & 612.3 & 718.3 & 612.1\end{array}$ 733.4

$\begin{array}{lllllllll}\text { GR } 611.9 & 748.2 & 612.1 & 761.1 & 611.9 & 778.5 & 611.0 & 791.3 & 610.8\end{array}$

804.3

$\begin{array}{lllllllll}\text { GR } 610.2 & 819.9 & 609.3 & 835.2 & 608.3 & 846.0 & 607.0 & 860.8 & 606.2\end{array}$ 873.0

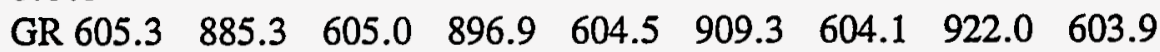
935.5

$\begin{array}{lllllllll}\text { GR } 603.9 & 946.8 & 604.0 & 958.3 & 604.1 & 970.5 & 604.0 & 985.6 & 604.2\end{array}$ 996.4

$\begin{array}{lllllllll}\text { GR } 604.2 & 1007.8 & 604.1 & 1019.9 & 604.0 & 1032.7 & 604.3 & 1044.4 & 604.7\end{array}$ 1060.1

$\begin{array}{lllllllll}\text { GR } 604.8 & 1068.3 & 604.7 & 1080.3 & 605.3 & 1093.7 & 605.8 & 1104.5 & 605.8\end{array}$ 1118.7

$\begin{array}{lllllllll}\text { GR } 605.6 & 1128.7 & 606.1 & 1143.7 & 606.6 & 1156.9 & 607.1 & 1169.5 & 607.0\end{array}$ 1182.1

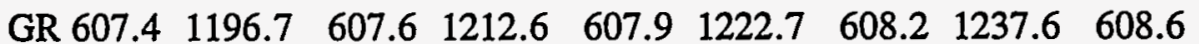
1249.4

$\begin{array}{lllllllll}\text { GR } 608.8 & 1261.4 & 609.0 & 1275.8 & 609.3 & 1287.0 & 609.5 & 1302.7 & 609.6\end{array}$ 1317.0

$\begin{array}{lllllllll}\text { GR } 609.9 & 1330.8 & 610.1 & 1347.1 & 610.3 & 1358.9 & 610.4 & 1375.7 & 610.2\end{array}$ 1387.2

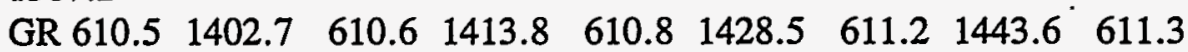
1459.4

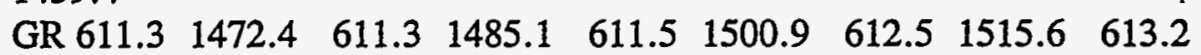
1530.4

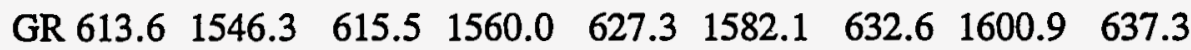
1653.0

GR 647.71675 .0

$\begin{array}{lllllllll}\mathrm{X} 1106.40 & 81 & 500.0 & 1408.9 & 2112 & 2112 & 2112 & .0000 & .00\end{array}$ $\begin{array}{lllllllll}\text { GR } 647.2 & 500.0 & 635.5 & 530.2 & 631.4 & 551.5 & 625.3 & 565.8 & 621.0\end{array}$ 584.4

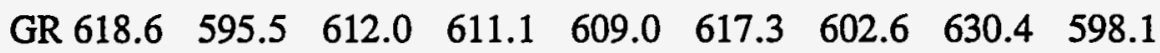


639.6

$\begin{array}{lllllllll}\text { GR 597.5 } & 648.8 & 596.9 & 657.4 & 596.1 & 666.0 & 595.2 & 675.2 & 595.2\end{array}$ 682.5

$\begin{array}{lllllllll}\text { GR } 595.3 & 689.9 & 595.3 & 699.5 & 595.3 & 708.5 & 595.3 & 714.9 & 595.5\end{array}$ 725.6

$\begin{array}{lllllllll}\text { GR } 596.0 & 732.5 & 597.0 & 744.1 & 597.4 & 749.3 & 598.2 & 757.4 & 599.0\end{array}$ 765.8

$\begin{array}{lllllllll}\text { GR } 599.3 & 771.6 & 599.7 & 784.3 & 599.9 & 797.2 & 599.9 & 802.5 & 599.7\end{array}$ 813.6

$\begin{array}{lllllllll}\text { GR } 599.8 & 820.2 & 600.5 & 831.2 & 600.9 & 837.5 & 601.5 & 847.5 & 601.8\end{array}$ 856.3

$\begin{array}{lllllllll}\text { GR } 601.9 & 865.5 & 602.2 & 874.6 & 602.8 & 883.3 & 603.0 & 893.9 & 603.4\end{array}$

903.9

$\begin{array}{lllllllll}\text { GR } 603.8 & 913.7 & 604.0 & 924.8 & 604.5 & 931.9 & 604.7 & 943.5 & 605.2\end{array}$ 952.5

$\begin{array}{lllllllll}\text { GR } 605.8 & 961.1 & 606.4 & 971.3 & 606.9 & 979.8 & 607.5 & 991.8 & 608.0\end{array}$ 1000.5

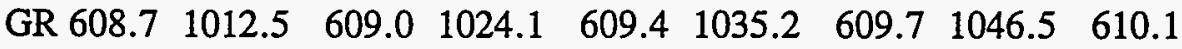
1055.5

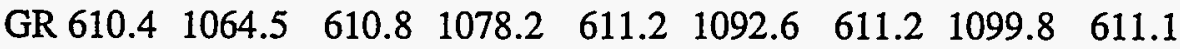
1112.4

$\begin{array}{lllllllll}\text { GR } 611.2 & 1122.8 & 611.2 & 1133.2 & 611.0 & 1145.7 & 610.8 & 1152.8 & 610.7\end{array}$ 1166.7

$\begin{array}{lllllllll}\text { GR } 610.6 & 1176.9 & 610.1 & 1189.8 & 609.8 & 1200.2 & 609.7 & 1210.2 & 609.3\end{array}$ 1219.6

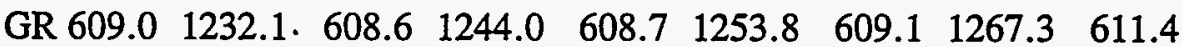
1274.3

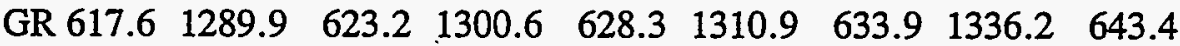
1375.8

GR 652.31408 .9

$\begin{array}{lllllllll}\mathrm{X} 1106.80 & 81 & 500.0 & 1634.0 & 2112 & 2112 & 2112 & .0000 & .00\end{array}$

$\begin{array}{lllllllll}\text { GR } 657.7 & 500.0 & 640.0 & 536.2 & 627.9 & 564.3 & 624.3 & 580.0 & 619.5\end{array}$

599.2

$\begin{array}{lllllllll}\text { GR } 616.2 & 614.0 & 615.3 & 627.0 & 615.3 & 641.4 & 615.1 & 654.0 & 614.7\end{array}$

669.5

$\begin{array}{lllllllll}\text { GR } 615.1 & 682.1 & 614.6 & 698.2 & 614.6 & 709.8 & 614.7 & 724.3 & 614.5\end{array}$

736.7

$\begin{array}{lllllllll}\text { GR } 614.5 & 750.0 & 614.4 & 763.6 & 614.3 & 774.9 & 614.6 & 791.5 & 615.0\end{array}$

802.6

$\begin{array}{lllllllll}\text { GR } 615.1 & 817.9 & 615.2 & 833.1 & 615.4 & 845.8 & 615.6 & 861.6 & 615.7\end{array}$

873.8

$\begin{array}{lllllllll}\text { GR } 615.8 & 891.2 & 615.9 & 901.8 & 616.1 & 920.6 & 616.1 & 930.0 & 616.7\end{array}$ 945.3

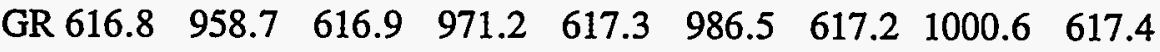
1015.2

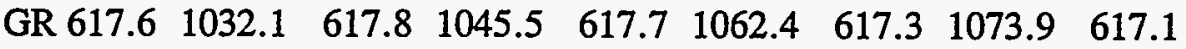
1087.3 


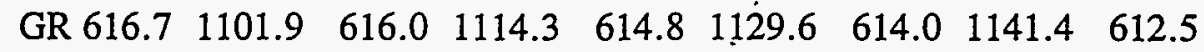
1156.3

$\begin{array}{llllllll}\text { GR } 611.1 .1169 .9 & 609.7 & 1183.0 & 608.3 & 1197.1 & 607.5 & 1206.4 & 606.6\end{array}$ 1218.7

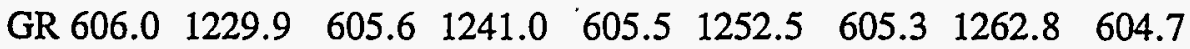
1275.1

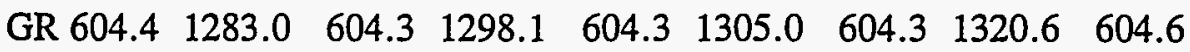
1328.4

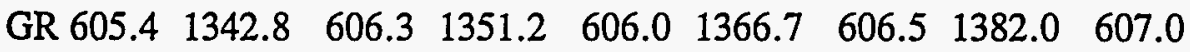
1389.0

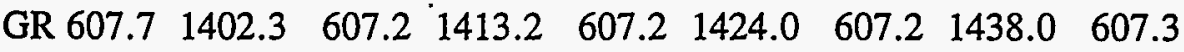
1445.8

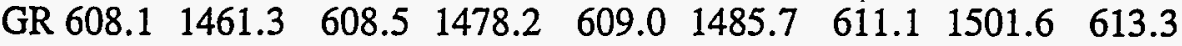
1510.4

$\begin{array}{lllllllll}\text { GR } 614.8 & 1524.0 & 619.3 & 1539.0 & 627.1 & 1559.8 & 633.9 & 1574.9 & 640.9\end{array}$ 1604.7

GR 649.81634 .0

$\begin{array}{lllllllll}\mathrm{X} 1107.00 & 81 & 500.0 & 2166.9 & 1056 & 1056 & 1056 & .0000 & .00\end{array}$

$\begin{array}{lllllllll}\text { GR } 649.1 & 500.0 & 632.3 & 534.6 & 610.9 & 570.2 & 605.1 & 588.0 & 602.1\end{array}$

604.6

$\begin{array}{lllllllll}\text { GR } 602.1 & 621.2 & 605.1 & 636.7 & 607.2 & 656.6 & 607.9 & 672.3 & 609.2\end{array}$

691.8

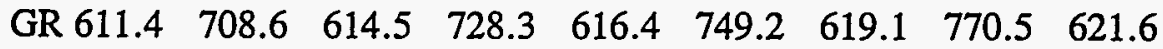

796.0

$\begin{array}{lllllllll}\text { GR } 623.3 & 825.8 & 623.2 & 850.2 & 622.9 & 873.0 & 622.0 & 901.0 & 620.9\end{array}$

928.1

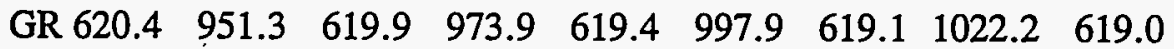

1046.6

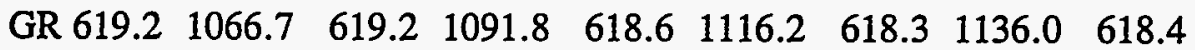
1160.9

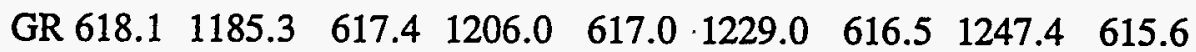
1272.8

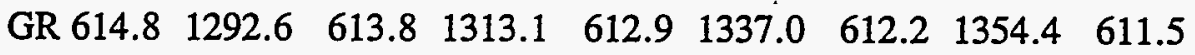
1374.4

$\begin{array}{lllllllll}\text { GR } 610.6 & 1393.1 & 610.4 & 1414.8 & 610.7 & 1431.5 & 611.0 & 1452.7 & 611.1\end{array}$ 1469.9

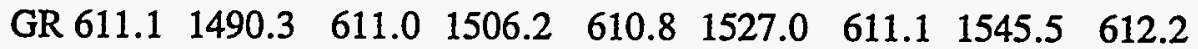
1563.5

$\begin{array}{lllllllll}\text { GR } 611.7 & 1585.1 & 610.1 & 1605.8 & 610.1 & 1623.6 & 609.5 & 1639.2 & 609.4\end{array}$ 1659.7

$\begin{array}{lllllllll}\text { GR } 609.2 & 1681.4 & 608.8 & 1697.3 & 608.3 & 1718.4 & 609.8 & 1732.8 & 610.9\end{array}$ 1751.8

$\begin{array}{lllllllll}\text { GR } 608.3 & 1770.3 & 607.2 & 1788.9 & 606.4 & 1806.4 & 606.3 & 1825.3 & 605.2\end{array}$ 1840.3

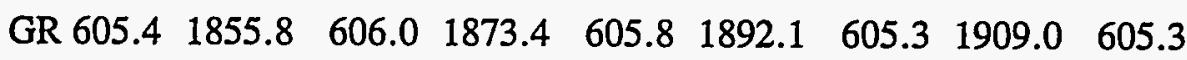
1929.1

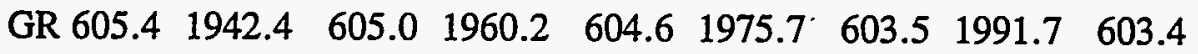


2008.0

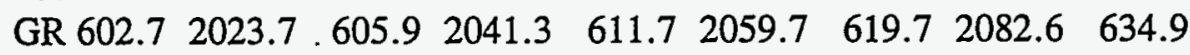
2120.9

GR 650.82166 .9

$\begin{array}{lllllllll}\mathrm{X} 1107.15 & 81 & 500.0 & 2618.3 & 792 & 792 & 792 & .0000 & .00\end{array}$

$\begin{array}{lllllllll}\text { GR } 656.3 & 500.0 & 631.8 & 554.5 & 615.6 & 597.1 & 611.6 & 619.5 & 607.8\end{array}$

650.2

$\begin{array}{lllllllll}\text { GR } 605.7 & 676.2 & 607.4 & 697.2 & 612.2 & 725.8 & 619.1 & 752.1 & 620.7\end{array}$

782.5

$\begin{array}{lllllllll}\text { GR } 617.3 & 812.2 & 614.3 & 842.1 & 612.4 & 867.3 & 611.3 & 892.7 & 610.5\end{array}$

919.9

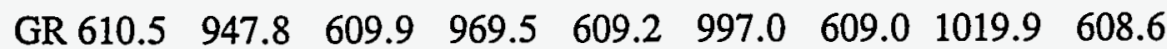
1044.4

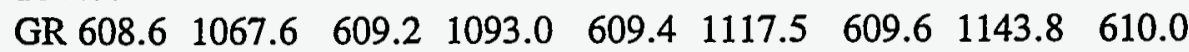
1168.4

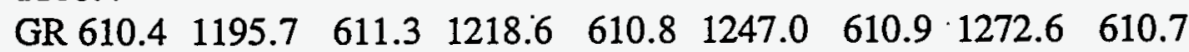
1298.1

$\begin{array}{lllllllll}\text { GR } 610.3 & 1321.1 & 610.0 & 1345.2 & 608.6 & 1371.7 & 609.0 & 1393.6 & 610.0\end{array}$ .1421 .0

$\begin{array}{lllllllll}\text { GR } 609.4 & 1447.7 & 609.6 & 1472.7 & 610.0 & 1499.4 & 610.5 & 1521.6 & 611.0\end{array}$ 1549.0

$\begin{array}{lllllllll}\text { GR } 610.3 & 1575.1 & 610.2 & 1601.6 & 609.1 & 1624.0 & 609.1 & 1645.8 & 608.7\end{array}$ 1672.3

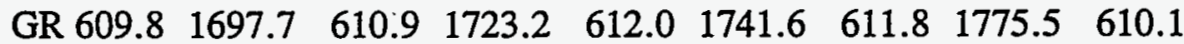
1802.5

GR $610.4 \quad 1826.9 \quad 611.4 \quad 1850.9 \quad 616.6 \quad 1880.2 \quad 620.5 \quad 1914.1 \quad 619.1$ 1941.1

$\begin{array}{lllllllll}\text { GR } 617.7 & 1973.1 & 616.0 & 2001.5 & 614.0 & 2028.0 & 612.8 & 2054.3 & 611.0\end{array}$ 2080.4

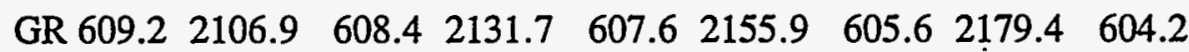
2200.9

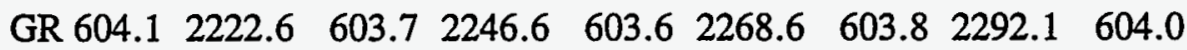
2316.3

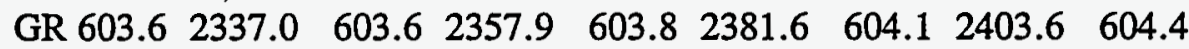
2427.9

$\begin{array}{lllllllll}\text { GR } 604.2 & 2451.8 & 603.9 & 2471.8 & 606.1 & 2498.4 & 616.6 & 2522.7 & 631.9\end{array}$ 2561.0

GR 652.92618 .3

NC $0.040 \quad 0.040 \quad 0.028 \quad 0.10 \quad 0.30$

$\begin{array}{lllllllll}\mathrm{X} 1107.42 & 81 . & 499.8 & 3775.7 & 1426 & 1426 & 1426 & .00000 & .00\end{array}$

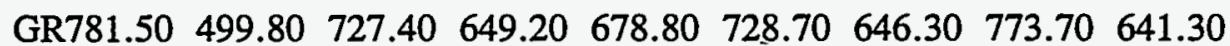
812.50

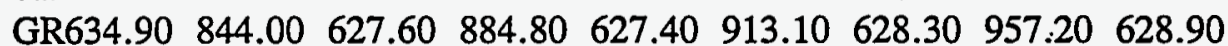
1003.30

GR629.50 1029.80 $629.401058 .80 \quad 629.801101 .60 \quad 629.201138 .00 \quad 629.20$ 
1173.30

GR629.00 $1209.40 \quad 626.801243 .60 \quad 624.50 \quad 1279.30 \quad 625.101314 .40 \quad 625.20$ 1350.20

GR625.30 1385.10 627.10 1419.40 629.20 $1453.90 \quad 629.401493 .80 \quad 628.90$ 1528.20

GR627.60 1566.50 $626.301600 .20 \quad 624.601633 .30 \quad 623.70 \quad 1668.20 \quad 621.70$ 1703.90

GR619.80 1734.00 $618.101770 .00615 .301804 .80 \quad 614.701835 .00 \quad 613.30$ 1866.10

GR612.60 1901.30 611.30 1934.10 612.10 1966.40 611.00 1996.10 610.60 2027.20

GR609.70 2063.90 610.50 2093.50 610.70 2127.70 $610.802158 .90 \quad 611.80$ 2187.50

GR612.40 2222.80 613.20 2255.10 612.70 2288.20 614.20 2318.10 617.50 2353.00

GR617.90 2387.90 616.30 2418.80 632.10 2454.60 648.00 2495.20 651.00 2535.40

GR653.10 2582.00 651.502620.10 651.20 2660.40 651.00 2701.80 651.50 2746.70

GR652.40 2786.60 651.40 2830.00 651.90 2866.80 654.20 2909.60 653.30 2956.00

GR653.40 2996.20 653.80 3037.80 653.60 3084.40 $652.903123 .20 \quad 652.80$ 3166.90

GR654.00 3207.70 $653.003253 .20 \quad 658.803295 .40 \quad 666.803342 .40 \quad 666.10$ 3385.60

GR673.00 $3433.30 \quad 678.803487 .30 \quad 690.103545 .80 \quad 704.703603 .40 \quad 719.20$ 3687.30

GR744.60 3775.70

* LOWER GRANITE DAM. RM 107.43

$\begin{array}{lllllllll}\mathrm{X} 1107.43 & 81 . & 499.8 & 3775.7 & 1 & 1 & 1 & .00000 & .00\end{array}$

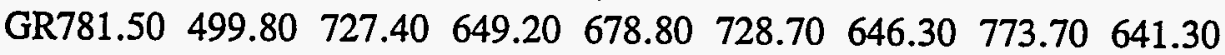
812.50

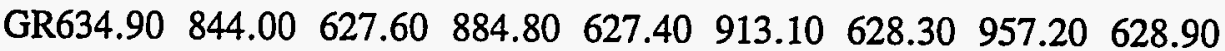
1003.30

GR629.50 1029.80 $629.401058 .80 \quad 629.801101 .60 \quad 629.201138 .00 \quad 629.20$ 1173.30

GR629.00 $1209.40 \quad 626.801243 .60 \quad 624.501279 .30 \quad 625.101314 .40 \quad 625.20$ 1350.20

GR625.30 1385.10 $627.101419 .40 \quad 629.201453 .90 \quad 629.401493 .80 \quad 628.90$ 1528.20

GR627.60 1566.50 $626.301600 .20 \quad 624.601633 .30 \quad 623.70 \quad 1668.20 \quad 621.70$ 1703.90

GR619.80 1734.00 618.10 1770.00 615.30 1804.80 614.70 $1835.00 \quad 613.30$ 1866.10

GR612.60 1901.30 611.30 1934.10 612.10 1966.40 611.00 1996.10 610.60 2027.20 
GR609.70 2063.90 610.50 2093.50 610.70 2127.70 $610.802158 .90 \quad 611.80$ 2187.50

GR612.40 2222.80 613.20 2255.10 612.70 2288.20 614.20 2318.10 617.50 2353.00

GR617.90 2387.90 616.302418.80 632.102454.60 648.00 2495.20 651.00 2535.40

GR653.10 2582.00 $651.502620 .10 \quad 651.202660 .40 \quad 651.002701 .80 \quad 651.50$ 2746.70

GR652.40 2786.60 $651.402830 .00 \quad 651.902866 .80 \quad 654.202909 .60 \quad 653.30$ 2956.00

GR653.40 2996.20 $653.803037 .80 \quad 653.603084 .40 \quad 652.903123 .20 \quad 652.80$ 3166.90

GR654.00 3207.70 $653.003253 .20 \quad 658.803295 .40 \quad 666.803342 .40 \quad 666.10$ 3385.60

GR673.00 3433.30 678.80 3487.30 690.10 3545.80 $704.703603 .40 \quad 719.20$ 3687.30

GR744.60 3775.70

$\begin{array}{lllllllll}\mathrm{X} 1107.73 & 81 . & 499.8 & 3775.7 & 1540.0 & 1600.0 & 1600.0 & .00000 & .00\end{array}$

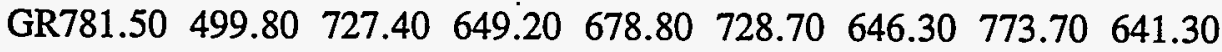
812.50

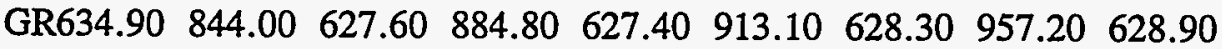
1003.30

GR629.50 1029.80 $629.401058 .80 \quad 629.801101 .60 \quad 629.201138 .00 \quad 629.20$ 1173.30

GR629.00 1209.40 $626.801243 .60 \quad 624.501279 .30 \quad 625.10 \quad 1314.40 \quad 625.20$ 1350.20

GR625.30 1385.10 627.10 1419.40 629.20 1453.90 629.40 $1493.80 \quad 628.90$ 1528.20

GR627.60 $1566.50 \quad 626.301600 .20 \quad 624.601633 .30 \quad 623.70 \quad 1668.20 \quad 621.70$ 1703.90

GR619.80 $1734.00618 .101770 .00 \quad 615.301804 .80 \quad 614.701835 .00 \quad 613.30$ 1866.10

GR612.60 1901.30 611.30 1934.10 612.10 1966.40 611.00 1996.10 610.60 2027.20

GR609.70 2063.90 610.50 2093.50 610.70 2127.70 610.80 2158.90 611.80 2187.50

GR612.40 2222.80 613.20 2255.10 612.70 2288.20 614.20 2318.10 617.50 2353.00

GR617.90 2387.90 $616.302418 .80 \quad 632.102454 .60 \quad 648.002495 .20 \quad 651.00$ 2535.40

GR653.10 2582.00 651.50 2620.10 651.20 2660.40 651.00 2701.80 651.50 2746.70

GR652.40 2786.60 $651.402830 .00651 .902866 .80 \quad 654.202909 .60 \quad 653.30$ 2956.00

GR653.40 2996.20 653.80 3037.80 $653.603084 .40 \quad 652.903123 .20 \quad 652.80$ 3166.90 
GR654.00 3207.70 653.00 3253.20 658.80 3295.40 666.80 $3342.40 \quad 666.10$ 3385.60

GR673.00 3433.30 678.80 3487.30 690.10 3545.80 704.70 3603.40 719.20 3687.30

GR744.60 3775.70

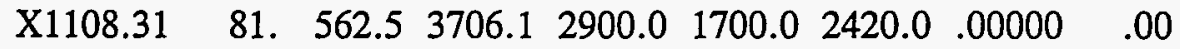

GR757.80 $562.50 \quad 711.50 \quad 661.60 \quad 701.30 \quad 770.60 \quad 690.50 \quad 827.70 \quad 680.90$ 887.20

GR676.70 $938.20 \quad 676.10989 .90 \quad 676.101039 .20 \quad 676.001089 .60 \quad 675.60$ 1138.80

GR674.50 1190.40 670.30 1235.10 659.80 1281.00 658.30 1325.10 656.90 1364.30

GR655.40 1404.20 654.40 1442.60 653.90 1482.30 653.50 1522.40 652.00 1561.80

GR652.10 $1601.60 \quad 650.701640 .00 \quad 634.301675 .70 \quad 625.401711 .80 \quad 627.30$ 1741.20

GR626.40 $1773.40 \quad 626.301803 .50 \quad 626.701833 .60625 .001866 .90 \quad 624.60$ 1900.90

GR622.90 1930.50 $622.801962 .10 \quad 621.201989 .50 \quad 620.502017 .10 \quad 619.90$ 2051.80

GR619.00 2085.70 619.20 2113.10 618.80 2140.50 618.60 2174.40 618.10 2207.70

GR619.20 2233.00 619.20 2263.10 619.80 2290.10 620.00 2323.20 621.50 2353.60

GR621.40 2383.60 623.20 2416.70 622.80 2450.40 623.40 2476.50 622.70 2506.50

GR621.70 2539.30 621.10 2572.20 620.70 2599.60 622.50 2631.80 624.30 2661.70

GR628.40 2691.90 634.20 2725.70 640.80 2763.40 643.30 2794.60 643.40 2833.00

GR644.20 2869.40 643.80 2906.40 649.102939.60 $650.302975 .60 \quad 648.20$ 3018.90

GR648.20 3057.80 647.10 3091.20 646.60 3130.30 647.80 3166.90 648.00 3205.10

GR647.40 $3244.20647 .403282 .00647 .703321 .00 \quad 647.203360 .60 \quad 652.70$ 3395.30

GR655.30 3435.20 $645.803474 .60 \quad 650.403512 .40 \quad 669.803551 .40 \quad 708.20$ 3633.30

GR743.70 3706.10

$\begin{array}{llllllll}\mathrm{X} 1111.24 & 81.499 .9 & 3573.6 & 12850.0 & 15550.0 & 14980.0 & .00000 & .00\end{array}$

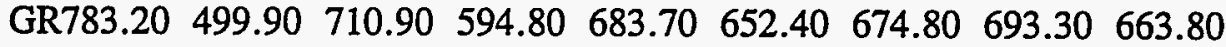
728.30

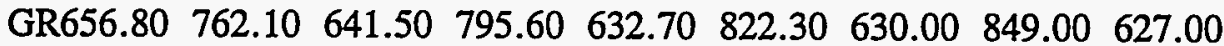
876.80

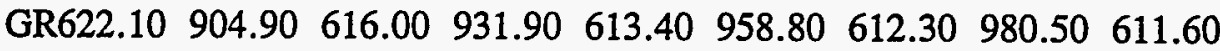


1008.70

GR610.50 $1035.90 \quad 610.801058 .10 \quad 610.701080 .60 \quad 611.301105 .60 \quad 612.80$ 1126.80

GR611.40 1157.50 $615.101186 .70 \quad 614.501208 .40 \quad 615.001230 .80 \quad 617.60$ 1258.30

GR616.30 1285.00 $617.801311 .20 \quad 618.601339 .40 \quad 618.001361 .10 \quad 619.10$ 1387.40

GR619.30 1414.00 $619.401440 .90 \quad 620.501467 .40 \quad 620.801494 .60 \quad 621.40$ 1518.60

GR622.30 1548.50 $622.201572 .40 \quad 622.301602 .80 \quad 623.701626 .00 \quad 624.30$ 1651.30

GR625.70 $1678.50 \quad 629.201711 .40 \quad 632.50 \quad 1734.80 \quad 642.101766 .10 \quad 655.50$ 1792.80

GR673.10 $1831.70 \quad 679.901869 .10 \quad 686.301913 .20 \quad 686.601955 .80 \quad 685.40$ 2000.70

GR686.10 2042.80 $687.002089 .50 \quad 687.502128 .90 \quad 688.102176 .60 \quad 687.30$ 2218.00

GR686.70 2256.20 687.00 2303.30 687.00 2350.80 687.50 2391.20 687.50 2436.80

GR688.10 2478.00 $688.702520 .00 \quad 689.002564 .40 \quad 689.402607 .90 \quad 691.20$ 2656.70

GR693.60 2707.40 694.00 2750.50 694.70 2794.70 696.10 2844.30 697.40 2897.70

GR699.20 2942.70 $701.302992 .40 \quad 703.703042 .00 \quad 707.203094 .80 \quad 710.40$ 3152.80

GR714.50 $3210.20 \quad 718.903273 .60 \quad 723.203342 .30 \quad 729.903410 .30 \quad 733.80$ 3483.70

GR745.70 3573.60

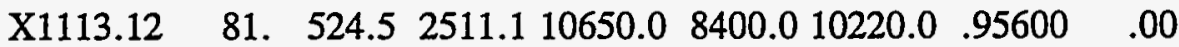

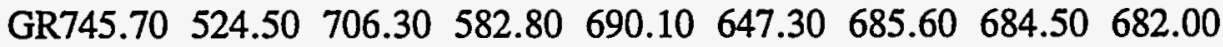
721.80

$\begin{array}{lllllll}\text { GR679.80 } & 757.40 \quad 678.50 \quad 790.50 & 677.60 & 824.20 & 675.60 & 857.30 & 674.40\end{array}$

891.90

GR673.00 $920.80 \quad 671.30 \quad 947.60 \quad 670.20 \quad 980.40 \quad 668.80 \quad 1013.20 \quad 667.70$ 1038.00

GR666.30 1068.60 665.30 1096.10 664.00 1124.00 $662.80 \cdot 1151.20 \quad 659.80$ 1178.80

GR645.40 $1202.60 \quad 637.701221 .70 \quad 631.301244 .60 \quad 628.601266 .90 \quad 627.50$ 1283.80

GR626.70 $1295.10626 .101324 .00 \quad 625.601344 .90 \quad 625.601363 .70 \quad 626.10$ 1381.60

GR625.30 $1402.40 \quad 625.201411 .40 \quad 625.201438 .10 \quad 624.601462 .00 \quad 624.30$ 1478.30

GR624.20 $1497.30 \quad 624.101516 .40 \quad 623.70 \quad 1526.60 \quad 623.501554 .50 \quad 623.50$ 1579.10

GR623.60 1592.60 $623.601612 .90 \quad 623.301628 .80 \quad 623.001640 .30 \quad 622.60$ 
1667.50

GR622.20 1690.40 622.10 1705.20 621.90 1730.60 621.90 1741.50 621.40 1758.60

GR621.50 1779.70 $622.001790 .70 \quad 621.301816 .40 \quad 620.801840 .80 \quad 621.10$ 1853.80

GR621.10 1878.70 $621.401891 .50 \quad 621.501912 .50 \quad 621.701928 .20^{\circ} \quad 621.60$ 1949.60

GR621.80 1966.40 $622.101988 .90 \quad 621.702002 .90 \quad 622.502022 .70 \quad 621.50$ 2039.90

GR621.50 2056.40 620.70 2078.60 619.70 2101.50 620.90 2114.90 623.60 2137.00

GR623.70 2153.70 625.10 2176.90 627.40 2191.30 637.80 2215.60 645.30 2233.80

GR661.00 2256.10 669.70 2286.10 673.80 2318.10 688.10 2352.70 733.20

2450.70

GR743.30 2511.10

$\begin{array}{lllllllll}\mathrm{X} 1114.92 & 81 . & 599.8 & 3071.4 & 9200.0 & 9300.0 & 9150.0 & .00000 & .00\end{array}$

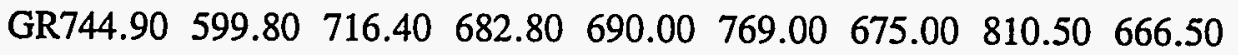

843.30

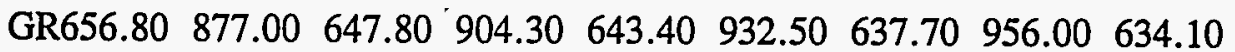

979.80

GR633.30 $1001.80631 .601028 .30630 .201049 .30 \quad 629.601070 .30 \quad 629.90$ 1095.00

GR628.40 1116.70628 .201141 .30628 .601162 .00629 .501184 .00629 .80 1208.50

GR629.70 $1231.00 \quad 630.001250 .30 \quad 629.801275 .20 \quad 629.70 \quad 1297.00 \quad 629.90$ 1322.80

GR629.80 1344.30 630.20 1368.30 630.10 1381.70 630.30 1414.00 630.70 1441.30

GR630.40 1459.90 630.80 1490.70 630.90 $1506.50 \quad 631.401527 .00 \quad 631.60$ 1553.00

GR632.10 $1585.00632 .501600 .80 \quad 632.801616 .30 \quad 632.701645 .60 \quad 633.40$ 1670.00

GR633.30 $1694.80 \quad 633.301720 .70 \quad 633.70 \quad 1740.30 \quad 634.40 \quad 1767.70 \quad 634.70$ 1787.30

GR636.20 $1812.00 \quad 637.901836 .80 \quad 639.40 \quad 1855.70 \quad 639.60 \quad 1884.70 \quad 640.00$ 1910.60

GR640.40 1935.50 640.90 1961.50 641.50 1985.50 $641.70 \quad 2010.20 \quad 642.20$ 2038.50

GR642.00 2060.30 642.802088.00 643.302116.50 644.30 2139.00 646.00 2167.20

GR646.60 2194.00 647.102220.40 647.90 2247.50 649.00 2275.60 649.70 2301.00

GR650.00 2327.50 $649.502356 .00 \quad 649.202382 .80 \quad 650.502411 .00 \quad 653.40$ 2442.60

GR662.20 2467.50 675.80 2502.70 681.10 2541.00 687.30 2583.40 691.30 
2629.00

GR693.10 2677.50 696.20 2724.00 $701.402782 .00 \quad 710.802839 .00 \quad 727.70$ 2972.60

GR744.50 3071.40

$\begin{array}{llllllll}X 1119.56 & 81.595 .0 & 2777.8 & 24700.0 & 23750.024500 .0 & .00000 & .00\end{array}$

GR749.70 $595.00 \quad 725.30 \quad 719.90 \quad 721.30 \quad 785.10 \quad 716.30 \quad 837.70 \quad 712.30$ •

892.20

GR707.40 $943.40703 .60981 .80 \quad 701.201019 .90698 .101053 .90695 .90$. 1088.60

GR693.00 $1125.60 \quad 690.301157 .50 \quad 687.60 \quad 1189.30 \quad 684.301220 .80 \quad 681.90$ 1248.60

GR679.40 $1277.90 \quad 677.501304 .50 \quad 674.301329 .90 \quad 671.801353 .60 \quad 669.40$ 1378.20

GR666.30 1401.90 663.90 1425.00 661.30 1449.10 $657.601471 .80 \quad 656.30$ 1491.90

GR654.00 $1511.80 \quad 652.801531 .70 \quad 652.001552 .70 \quad 650.601573 .10 \quad 650.30$ 1595.10

GR649.60 $1610.40 \quad 649.101631 .00648 .301648 .80 \quad 647.201666 .50 \quad 646.90$ 1689.00

GR645.70 $1713.30644 .901726 .30644 .701748 .40 \quad 644.301764 .70 \quad 643.40$ 1785.00

GR642.80 $1801.60642 .401819 .10 \quad 641.401836 .90 \quad 641.201854 .90 \quad 640.80$ 1872.70

GR640.60 1890.80 $640.201908 .10640 .101925 .00 \quad 640.301945 .70 \quad 640.30$ 1960.50

GR640.60 1981.70 $640.501996 .60640 .702017 .50 \quad 641.002031 .80 \quad 641.40$ 2053.10

GR641.70 2068.70 $640.802089 .50 \quad 641.302103 .80 \quad 642.302125 .10 \quad 643.30$ 2140.00

GR643.20 2163.90 $643.802187 .80 \quad 645.602201 .20 \quad 647.402223 .60 \quad 649.00$ 2238.30

GR651.90 2257.80 $661.202279 .40 \quad 667.402304 .30 \quad 670.002326 .90 \quad 671.60$ 2349.30

GR673.30 2374.40 679.602404.60 684.70 2430.20 686.50 2459.00 684.90 2490.40

GR691.60 2524.10 702.40 2554.10 706.40 2596.20 $713.502643 .70 \quad 720.70$ 2710.50 .

GR742.60 2777.80

$\begin{array}{llllllllll}\mathrm{X} 1120.46 & 81 . & 499.8 & 2858.8 & 4150.0 & 4850.0 & 4610.0 & .96400 & .00\end{array}$

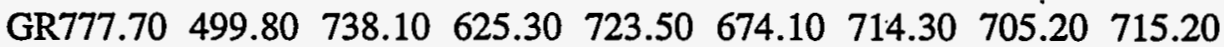
755.70

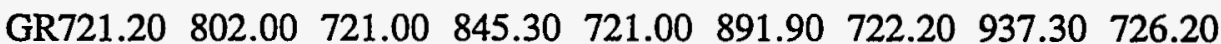

986.70

GR725.80 $1033.80 \quad 725.001080 .40 \quad 723.201127 .90 \quad 721.701175 .80 \quad 722.70$ 1221.90 
GR724.60 1265.70 $715.701313 .90710 .701356 .40 \quad 702.601391 .30 \quad 699.70$ 1426.00

GR697.20 1457.70 $695.101489 .40688 .001521 .40 \quad 683.101548 .70 \quad 677.30$ 1576.10

GR672.10 1602.50 669.00 1626.10 666.60 $1649.70 \quad 664.90 \quad 1675.10 \quad 663.40$ 1695.80

GR661.70 $1722.10 \quad 660.601745 .50 \quad 659.201766 .60 \quad 657.701790 .00 \quad 656.30$ 1810.40

GR654.40 $1831.10 \quad 652.201854 .40 \quad 650.301872 .80 \quad 648.001895 .00 \quad 646.00$ 1917.40

GR644.10 1937.30 641.70 1960.40 640.30 1978.10 639.70 1997.10 638.30 2016.70

GR637.30 2035.80 636.302054.90 635.80 2073.70 634.80 2092.50 633.80 2107.30

GR632.60 2129.80 631.20 2150.00 630.50 2165.60 630.20 2186.30 630.20 2203.60

GR631.30 2223.70 632.802239.80 633.80 2259.90 635.80 2277.30 638.00 2295.30

GR638.90 2316.10 640.00 2335.30 642.30 2354.90 644.80 2375.20 650.10 2396.50

GR660.40 2422.60 664.902441.30 667.70 2466.40 671.10 2487.00 676.60 2514.10

GR680.80 2541.80 682.102569.40 683.70 2597.40 681.90 2622.90 680.60 2651.40

GR678.10 2676.50 686.30 2703.90 689.60 2737.40 $701.802767 .40 \quad 717.20$ 2805.20

GR744.00 2858.80

$\begin{array}{lllllllll}\mathrm{X} 1121.42 & 81 . & 542.5 & 2045.7 & 5450.0 & 5300.0 & 5290.0 & .00000 & .00\end{array}$

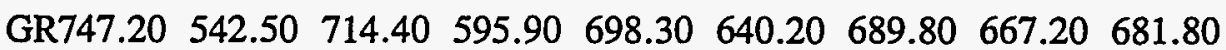
693.50

GR674.90 $713.70 \quad 668.70 \quad 734.30 \quad 665.30 \quad 751.00 \quad 661.20 \quad 770.20 \quad 657.20$ 784.20

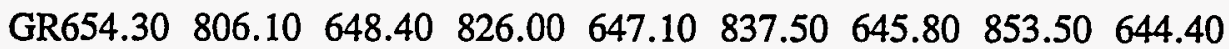
867.90

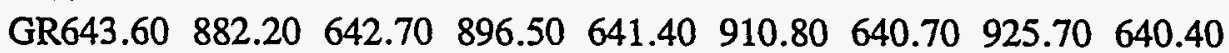
934.20

GR639.90 $954.20 \quad 639.70 \quad 962.60 \quad 639.60 \quad 982.80 \quad 639.30 \quad 999.50 \quad 639.00$ 1012.20

GR639.00 $1025.90 \quad 639.001041 .30 \quad 638.501057 .80 \quad 638.201069 .20 \quad 638.20$ 1080.70

GR637.80 $1097.10 \quad 637.601114 .10637 .501126 .10 \quad 637.001138 .00636 .30$ 1152.90

GR636.00 $1167.90635 .701181 .30 \quad 635.201196 .80 \quad 635.501208 .30 \quad 635.40$ 1218.70

GR635.40 $1236.70635 .501256 .10635 .601264 .00 \quad 635.601279 .00 \quad 636.80$ 1292.00 
GR637.40 1305.80 638.90 1320.40 641.10 1335.40 642.10 1347.30 643.70 1363.20

GR648.60 1379.20 $651.901391 .10 \quad 651.001410 .10 \quad 651.101428 .00 \quad 651.10$ 1442.00

GR651.30 1456.90 $651.701474 .40 \quad 651.901491 .50 \quad 652.101506 .00 \quad 652.10$ 1521.70

GR652.90 $1537.70 \quad 653.40 \quad 1550.60 \quad 653.20 \quad 1570.50 \quad 652.30 \quad 1584.20 \quad 651.50$ 1602.70

GR654.60 1618.00 $656.801635 .80660 .701652 .60 \quad 672.801673 .20685 .60$ 1693.60

GR692.90 $1719.80693 .801745 .60 \quad 689.00 \quad 1773.40 \quad 686.201798 .30 \quad 686.80$ 1821.20

GR687.60 1849.20 $688.301873 .00 \quad 691.00 \quad 1901.40 \quad 704.901929 .30 \quad 723.50$ 1982.20

GR747.50 2045.70

$\begin{array}{lllllllll}\mathrm{X} 1122.69 & 81 . & 500.4 & 1898.1 & 5100.0 & 5300.0 & 5230.0 & .00000 & .00\end{array}$

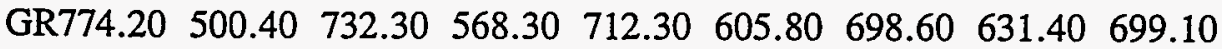
654.50

GR698.10 $676.00 \quad 696.20 \quad 700.40 \quad 694.80 \quad 719.50 \quad 693.30 \quad 745.80 \quad 691.90$

768.60

GR690.80 $787.80 \quad 690.20 \quad 807.10 \quad 687.80 \quad 829.70 \quad 681.90 \quad 851.00 \quad 675.80$

869.40

GR670.90 $887.10 \quad 668.90 \quad 903.70 \quad 667.20 \quad 920.80 \quad 665.70 \quad 937.80 \quad 664.50$ 955.30

GR662.40 $969.70 \quad 658.40 \quad 989.00656 .801002 .40 \quad 654.601020 .10 \quad 652.40$ 1032.40

GR649.80 1050.60 $647.901062 .30646 .101079 .90 \quad 644.801090 .30643 .50$ 1105.80

GR643.20 1118.30 643.20 1132.90 643.20 1145.00 643.20 1160.70 643.00 1173.90

GR643.40 $1187.10 \quad 643.301200 .90 \quad 642.80 \quad 1210.40 \quad 642.60 \quad 1228.20 \quad 642.40$ 1240.50

GR642.00 $1256.60641 .701272 .90 \quad 641.601284 .50 \quad 640.801300 .80 \quad 641.00$ 1312.10

GR640.90 $1326.50 \quad 640.401337 .70 \quad 639.901350 .90 \quad 639.601366 .20 \quad 639.50$ 1380.50 .

GR639.10 1392.60 639.00 1408.40 639.00 1419.40 638.90 1435.10 638.80 1447.20

GR638.80 1463.60 $639.001474 .40 \quad 640.901489 .30 \cdot 640.401500 .70 \quad 640.20$ 1516.10

GR640.80 $1528.60 \quad 641.101540 .90 \quad 643.10 \quad 1555.10 \quad 642.601567 .80 \quad 642.50$ 1583.10

GR644.70 $1594.60646 .601611 .40 \quad 648.201626 .90 \quad 647.501638 .80 \quad 648.20$ 1655.40

GR653.00 1668.50 $660.601687 .90666 .501701 .00 \quad 677.101722 .00681 .60$ 1736.10 
GR692.00 1755.10 693.00 1779.20 697.90 1804.30 $710.401827 .90 \quad 725.00$ 1859.30

GR745.00 1898.10

$\begin{array}{lllllllll}\mathrm{X} 1123.30 & 81 . & 537.1 & 2178.9 & 5050.0 & 4700.0 & 4860.0 & .00000 & .00\end{array}$

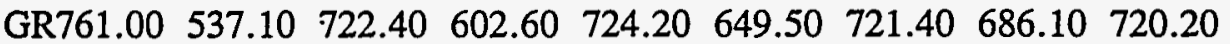
729.20

GR720.00 761.50 718.50 803.90 $716.80 \quad 840.40 \quad 714.30 \quad 873.10 \quad 711.50$ 907.30

GR707.70 $939.80 \quad 704.30 \quad 968.20700 .40 \quad 996.10 \quad 697.00 \quad 1018.80 \quad 691.80$ 1046.90

GR686.60 $1073.80683 .001092 .30 \quad 679.601114 .60 \quad 676.301130 .60 \quad 673.40$ 1148.50

GR670.40 $1168.70668 .601183 .30 \quad 664.801204 .20 \quad 662.901218 .80 \quad 659.90$ 1239.10

GR656.40 $1260.40 \quad 654.601272 .40 \quad 653.301288 .40 \quad 652.101303 .80 \quad 650.80$ 1316.80

GR649.40 $1332.70 \quad 648.201350 .00 \quad 647.501363 .00 \quad 646.501379 .50 \quad 645.80$ 1391.90

GR645.20 $1408.70644 .901422 .10644 .801436 .30 \quad 643.501451 .20 \quad 641.80$ 1465.80

GR640.70 1479.50 $640.301494 .60 \quad 638.801507 .50 \quad 637.30 \quad 1521.60 \quad 636.30$ 1534.60

GR636.30 $1550.30 \quad 634.801561 .30 \quad 633.70 \quad 1572.70 \quad 633.80 \quad 1588.70 \quad 633.20$ 1604.10

GR632.40 $1615.10631 .201627 .50 \quad 630.401639 .90 \quad 630.40 \quad 1652.40 \quad 633.60$ 1667.30

GR636.20 $1680.90638 .501694 .20640 .601708 .60 \quad 643.301720 .80 \quad 647.70$ 1736.40

GR652.20 $1751.10 \quad 653.501769 .30 \quad 654.601782 .50 \quad 655.70 \quad 1800.30 \quad 656.60$ 1814.20

GR657.30 1827.00660 .801845 .60663 .701859 .20666 .801881 .20670 .10 1898.90

GR672.30 1918.30 $675.601939 .50 \quad 677.301958 .10680 .601981 .00 \quad 681.50$ 1998.20

GR683.80 2018.90 693.90 2041.80 695.70 2070.10 $704.502092 .60 \quad 722.10$ 2128.50

GR746.80 2178.90

$\begin{array}{lllllllll}\mathrm{X} 1124.94 & 81 . & 563.7 & 2029.6 & 4800.0 & 6350.0 & 5660.0 & .98500 & .00\end{array}$

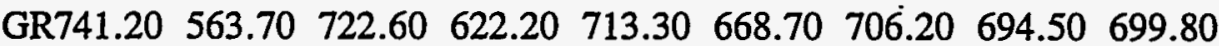
727.40

GR695.40 $756.10 \quad 690.90 \quad 775.50 \quad 682.00 \quad 797.20 \quad 680.10 \quad 814.60 \quad 676.50$ 832.00

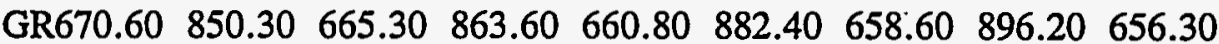
910.50

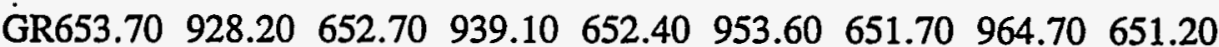


976.00

GR650.90 $992.50 \quad 650.40 \quad 1007.40 \quad 650.00 \quad 1017.90 \quad 649.70 \quad 1033.70 \quad 650.20$ 1045.50

GR649.20 1060.20 $648.901072 .70 \quad 648.901084 .60 \quad 648.401099 .10 \quad 648.30$ 1108.60

GR648.00 1123.30 $647.501136 .30 \quad 646.801149 .70 \quad 645.701163 .40 \quad 644.70$ 1175.60

GR644.10 $1188.80 \quad 643.801201 .30 \quad 642.901216 .60 \quad 642.601226 .30 \quad 642.60$ 1242.10

GR641.90 1250.60 $641.201262 .50 \quad 640.401274 .90 \quad 639.401288 .10 \quad 639.00$ 1299.90

GR638.40 $1315.30 \quad 638.701323 .00 \quad 641.10 \quad 1337.20 \quad 642.301347 .70 \quad 643.20$ 1361.60

GR643.80 1372.10 645.20 1381.60 644.90 $1398.00 \quad 645.201415 .40 \quad 646.50$ 1423.90

GR649.10 $1438.40 \quad 650.801449 .80 \quad 651.301461 .80 \quad 651.901475 .90 \quad 653.50$ 1485.10

GR655.70 $1502.80 \quad 657.80 \quad 1517.20 \quad 659.60 \quad 1532.30 \quad 661.301544 .80 \quad 663.90$ 1561.40

GR667.70 $1577.00674 .601596 .30682 .001617 .80686 .201632 .60 \quad 690.40$ 1654.40

GR692.10 1678.00 $695.101705 .10697 .30 \quad 1724.40 \quad 699.40 \quad 1752.00 \quad 701.30$ 1775.10

GR706.90 $1803.70 \quad 716.401837 .40 \quad 713.80 \quad 1878.20 \quad 706.201907 .10 \quad 731.10$ 1958.30

GR747.30 2029.60

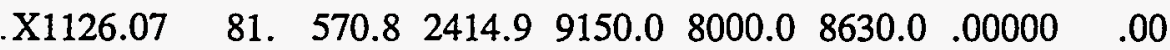

GR754.90 $570.80 \quad 717.00 \quad 642.70 \quad 707.60 \quad 683.30 \quad 702.90 \quad 712.20 \quad 695.50$

746.00 .

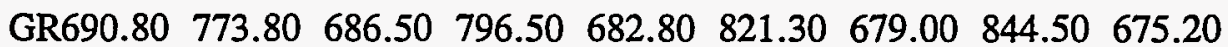

865.80

GR671.40 $887.00 \quad 668.10 \quad 904.70 \quad 664.00 \quad 928.00 \quad 661.10 \quad 950.70 \quad 659.30$

964.80

GR656.90 $985.00 \quad 655.90 \quad 999.00 \quad 654.70 \quad 1019.30 \quad 654.00 \quad 1033.80 \quad 652.80$ 1052.30

GR652.70 $1067.50 \quad 652.501087 .70 \quad 651.80 \quad 1102.30 \quad 652.001122 .30 \quad 652.00$ 1134.60

GR652.10 $1156.30 \quad 652.301168 .40 \quad 652.601184 .00 \quad 652.601202 .00 \quad 652.50$ 1218.00

GR652.70 $1236.00 \quad 653.201257 .70 \quad 653.401270 .80653 .601291 .00653 .60$ 1304.50

GR654.00 1321:00 654.501338 .00654 .501358 .00654 .801372 .30655 .60 1393.30

GR656.00 1408.00 $656.801428 .30 \quad 657.30 \quad 1442.40 \quad 658.401463 .00 \quad 660.90$ 1476.80

GR666.10 $1496.30 \quad 665.301514 .00 \quad 664.701531 .70 \quad 663.201552 .80 \quad 663.10$ 
1571.00

GR663.20 $1589.80 \quad 663.201609 .70 \quad 663.801628 .50 \quad 664.201642 .30 \quad 664.90$ 1667.00

GR665.20 1690.00 665.60 1704.00 665.80 1724.00 666.40 1743.30 667.10 1762.00

GR667.70 1781.00 $668.101801 .30 \quad 668.501820 .50 \quad 669.501840 .30 \quad 670.40$ 1861.00

GR671.30 1881.00 $672.301901 .30 \quad 673.501920 .50 \quad 675.501945 .30 \quad 677.30$ 1965.50

GR683.70 1987.00 690.902015.30 695.80 2041.50 704.50 2071.20 703.60 2103.80

GR703.80 2135.70 $706.902170 .50 \quad 708.302209 .00 \quad 710.002241 .00 \quad 745.70$ 2301.00

GR746.30 2414.90

$\begin{array}{lllllllll}\mathrm{X} 1127.03 & 81 . & 563.3 & 2503.8 & 5300.0 & 4250.0 & 5000.0 & .00000 & .00\end{array}$

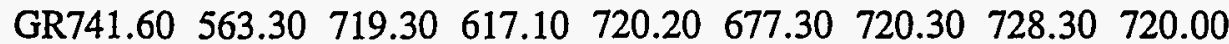
776.50

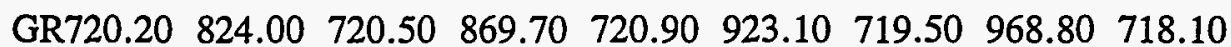
1010.50

GR715.70 $1053.10 \quad 712.901094 .10 \quad 711.201133 .50 \quad 710.00 \quad 1170.80 \quad 709.20$ 1205.40

GR708.00 1240.50 703.50 1271.80 697.30 $1300.30 \quad 691.601323 .90 \quad 687.70$ 1347.70

GR686.90 1371.40 $685.801395 .30684 .801416 .10 \quad 683.901435 .90 \quad 682.50$ 1458.80

GR681.20 1482.90 $680.401498 .20679 .101518 .60 \quad 677.701538 .40 \quad 676.40$ 1556.90

GR675.00 1578.80 674.10 1597.60 673.00 1614.20 671.80 1632.60 670.90 1652.10

GR670.00 1667.00669 .101687 .80668 .001702 .00667 .401720 .30666 .60 1737.10

GR666.10 $1753.80 \quad 665.401771 .10664 .801788 .90664 .601806 .50664 .10$ 1820.70

GR663.50 $1841.50 \quad 663.101854 .90 \quad 662.701871 .80 \quad 662.40 \quad 1886.40 \quad 662.10$ 1899.70

GR662.30 1919.20 $662.401933 .80 \quad 662.50 \quad 1951.60 * 662.301968 .80 \quad 662.30$ 1983.60

GR662.60 1999.30 662.602014.90 662.80 2028.70 662.90 2047.70 662.90 2064.40

GR663.20 2081.60 663.90 2099.40 664.30 2114.00 664.90 2133.40 665.30 2147.70

GR665.80 2167.50 $666.402180 .20 \quad 667.102196 .60 \quad 667.502215 .40 \quad 667.10$ 2232.80

GR666.60 2247.40 $664.302262 .00663 .002282 .80 \quad 663.902299 .00 \quad 667.40$ 2315.50

GR674.60 2336.40 690.00 2354.30 701.50 2383.50 $704.402406 .40 \quad 715.10$ 
2454.80

GR744.50 2503.80

$\begin{array}{lllllllll}\mathrm{X} 1127.63 & 81 . & 639.1 & 2505.3 & 2800.0 & 3150.0 & 3120.0 & .00000 & .00\end{array}$

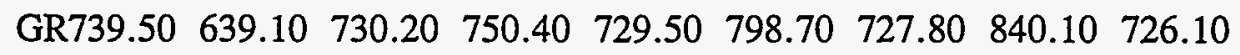
914.80

GR725.20 971.00 724.20 1019.50 723.90 $1069.60721 .301119 .20 \quad 719.30$ 1162.40

GR718.80 1204.60 717.60 1243.30 $716.201282 .90 \quad 713.801321 .70 \quad 710.50$ 1356.90

GR706.60 1390.10 $702.701417 .90 \quad 699.701439 .40 \quad 695.801468 .40 \quad 692.60$ 1490.60

GR690.50 1511.90 $687.801531 .20684 .901553 .30 \quad 683.501570 .30 \quad 679.50$ 1593.40

GR677.20 $1615.60675 .701629 .90673 .401649 .70 \quad 672.101663 .90 \quad 669.70$ 1683.70

GR668.40 1696.10 667.40 1711.90 666.90 1728.40 666.30 1745.30 665.90 1759.40

GR665.40 $1773.00 \quad 664.901788 .70 \quad 664.301807 .40 \quad 663.801819 .80 \quad 663.30$ 1833.60

GR662.60 $1850.40 \quad 662.40 \quad 1866.30 \quad 661.901879 .60 \quad 661.50 \quad 1893.70 \quad 660.50$ 1907.70

GR659.90 1921.90 659.50 1936.70 659.00 1951.90 658.60 1966.00 658.10 1982.40

GR657.40 1993.80 657.20 2005.10 656.70 2023.00 656.30 2039.40 655.50 2049.30

GR655.00 2061.80 654.00 2076.70 653.40 2089.20 652.70 2104.40 652.20 2119.60

GR651.80 2131.00 650.80 2143.10 650.40 2157.00 649.80 2175.40 649.60 2183.20

GR648.80 2197.60 648.80 2207.60 648.70 2217.40 648.30 2234.30 647.60 2247.20

GR648.80 2260.10 $651.802275 .10 \quad 657.302285 .90 \quad 666.002303 .80 \quad 671.70$ 2316.90

GR677.70 2330.10 690.10 2353.80 $704.702381 .60 \quad 709.702409 .90 \quad 716.70$ 2449.40

GR744.80 2505.30

$\begin{array}{lllllllll}\mathrm{X} 1128.27 & 81 . & 610.0 & 2088.1 & 2800.0 & 3300.0 & 3110.0 & .00000 & .00\end{array}$

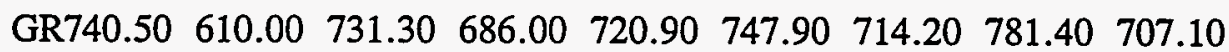
815.30

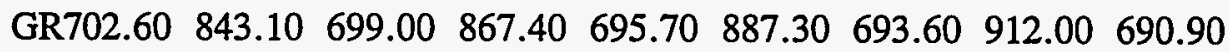
935.80

GR689.10 $954.30 \quad 687.70 \quad 973.70 \quad 686.50 \quad 991.80 \quad 685.70 \quad 1009.40 \quad 685.00$ 1029.50

GR684.20 $1043.70 \quad 682.90 \quad 1067.20 \quad 682.30 \quad 1090.30 \quad 681.40 \quad 1103.40 \quad 680.60$ 1119.80 
GR679.60 1137.60 678.60 1160.80 677.80 1172.40 676.90 1192.60 676.00 1205.10

GR674.40 1222.20 673.60 1237.00 673.00 1252.60 672.20 $1267.80 \quad 672.00$ 1282.10

GR671.70 1299.80 671.10 1316.80 670.10 $1330.70 \quad 668.801347 .90 \quad 668.80$ 1359.80

GR668.50 1371.60 $665.801389 .60 \quad 666.201407 .40 \quad 665.001417 .90663 .80$ 1431.30

GR663.50 $1446.10663 .401460 .00 \quad 663.101474 .10 \quad 662.601489 .80 \quad 662.70$ 1501.10

GR662.20 1512.10 $662.301529 .40 \quad 660.801548 .30 \quad 660.901557 .20661 .50$ 1572.10

GR662.30 $1583.40 \quad 663.001594 .90663 .101610 .50 \quad 662.901619 .60 \quad 661.80$ 1639.50

GR660.90 1657.90 660.20 1666.50 659.30 1681.60 658.50 1693.60 658.10 1705.40

GR658.00 1719.10 $657.401728 .30 \quad 657.60 \quad 1745.60 \quad 657.70 \quad 1758.50 \quad 657.60$ 1771.40

GR656.50 $1789.80656 .601796 .80656 .901813 .60 \quad 658.801823 .90 \quad 661.00$ 1834.90

GR662.00 $1850.50662 .001865 .30 \quad 662.301878 .10 \quad 666.601895 .90 \quad 674.50$ 1907.80

GR685.20 $1925.80691 .701943 .40 \quad 700.201967 .00 \quad 713.901994 .50 \quad 717.20$ 2036.30

GR744.10 2088.10

$\begin{array}{lllllllll}\mathrm{X} 1128.87 & 81 . & 565.9 & 1954.1 & 2300.0 & 3150.0 & 3020.0 & .00000 & .00\end{array}$

GR753.60 $565.90 \quad 715.30 \quad 600.90 \quad 703.20 \quad 635.10 \quad 700.00 \quad 655.00 \quad 697.70$ 678.30

GR692.40 $696.00 \quad 686.30 .718 .50 \quad 683.80 \quad 734.30 \quad 679.40 \quad 749.80 \quad 674.80$ 765.60

GR672.80 $780.50 \quad 670.70 \quad 795.80 \quad 669.00 \quad 811.50 \quad 667.30 \quad 826.00 \quad 664.90$ 837.90

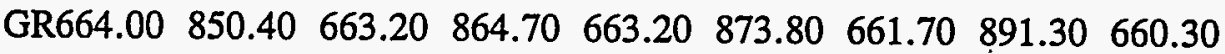
898.80

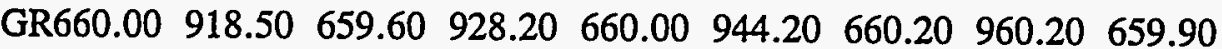
969.60

GR659.90 $983.60660 .30995 .60660 .801007 .00 \quad 661.001021 .30 \quad 661.00$ 1035.30

GR661.60 1047.80 661.901066.40 661.801074.40 662.30 1091.90 662.50 1100.30

GR662.90 1115.30 663.301126.80 663.70 1138.80 663.90 1152.60 663.80 1164.70

GR664.30 1181.20 664.801190.60 665.60 1208.60 666.40 1227.10 666.70 1236.30

GR667.40 $1251.00668 .001263 .50668 .901275 .50 \quad 669.101292 .40669 .90$ 1309.40 
GR670.20 1320.90 $670.60 \quad 1332.40 \quad 671.30 \quad 1350.30 \quad 671.801368 .30 \quad 672.20$ 1377.50

GR672.50 1394.70 $671.801407 .40 \quad 670.701422 .60 \quad 672.201436 .60 \quad 673.50$ 1449.80

GR675.00 1468.30 676.50 $1484.00 \quad 677.601498 .60 \quad 678.401515 .90 \quad 678.40$ 1530.90

GR678.30 1548.20 679.40 1562.10 683.90 $1581.80 \quad 686.701596 .60 \quad 690.90$ 1619.10

GR694.40 $1632.70 \quad 700.001653 .00 \quad 706.301676 .60713 .301706 .60 \quad 712.00$ 1731.50

GR712.10 $1761.60715 .601787 .60720 .801823 .00 \quad 720.801849 .70 \quad 735.70$ 1884.30

GR738.20 1954.10

$\begin{array}{lllllllll}\mathrm{X} 1129.27 & 81 . & 575.5 & 2464.5 & 2150.0 & 2100.0 & 2120.0 & .00000 & .00\end{array}$

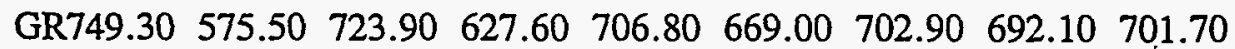

718.30

GR698.90 $747.50 \quad 696.70 \quad 766.10 \quad 693.00 \quad 789.00 \quad 691.70 \quad 807.30 \quad 690.80$ 824.60

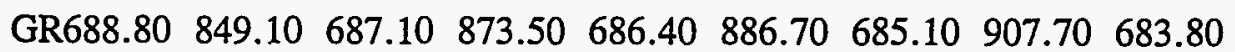
922.50

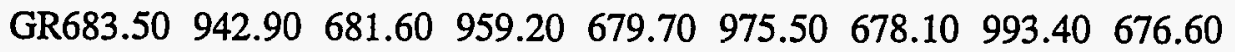
1007.70

GR675.30 1028.10 674.20 1042.20 672.90 $1058.80 \quad 671.301077 .10 \quad 670.80$ 1090.90

GR670.20 $1106.60 \quad 668.301121 .70 \quad 664.301136 .10 \quad 662.50 \quad 1150.30 \quad 660.70$ 1163.90

GR660.60 $1179.00 \quad 660.401193 .70 \quad 659.101206 .70 \quad 658.20 \quad 1223.30 \quad 657.20$ 1232.80

GR655.20 $1246.40 \quad 655.801258 .40 \quad 656.601270 .60 \quad 656.101286 .60 \quad 656.90$ 1305.30

GR657.10 $1312.30 \quad 658.301327 .90 \quad 659.201339 .40 \quad 660.001351 .50 \quad 660.40$ 1366.40

GR661.00 1380.20 $662.601395 .20664 .601409 .90 \quad 666.301424 .40668 .00$ 1439.20

GR669.60 1453.30 $671.501467 .60673 .801484 .80 \quad 675.701502 .90 \quad 677.40$ 1517.20

GR679.40 1531.10 $682.601551 .90685 .201571 .50687 .801589 .80 \quad 690.40$ 1611.20

GR692.80 1628.60 $696.601652 .80 \quad 699.201675 .20 \quad 702.901698 .80 \quad 706.80$ 1724.20

GR710.30 $1755.40713 .001780 .10716 .101815 .20 \quad 718.501846 .40720 .40$ 1886.70

GR721.10 1920.90 721.50 1961.90 $721.901996 .30 \quad 722.402036 .00 \quad 724.30$ 2077.30

GR725.60 2117.90 726.802163 .80728 .402216 .10733 .302261 .20738 .90 2301.20 
GR751.00 2464.50

$\begin{array}{lllllllll}\mathrm{X} 1130.00 & 81 . & 594.9 & 2327.5 & 4250.0 & 3000.0 & 3880.0 & .00000 & .00\end{array}$

GR741.40 $594.90 \quad 732.20 \quad 687.60 \quad 720.30 \quad 754.00 \quad 713.20 \quad 785.20 \quad 698.00$ 817.00

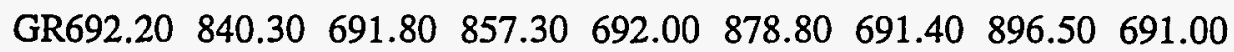
915.00

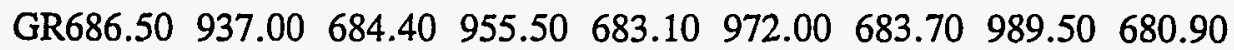
1006.00

GR678.60 1023.50 $678.401037 .30 \quad 677.501053 .70 \quad 676.401071 .00 \quad 676.50$ 1083.00

GR675.00 1101.00 674.00 1120.00. $673.001131 .10 \quad 671.001144 .00668 .80$ 1159.00

GR666.30 1173.30 $664.801186 .80 \quad 663.501204 .00 \quad 663.401214 .30 \quad 663.50$ 1228.00

GR662.50 1239.50 661.30 1252.00 660.00 1265.60 659.90 1276.00 659.90 1290.30

GR660.20 1304.00 $660.201316 .50 \quad 660.201332 .50 \quad 660.901341 .30 \quad 661.90$ 1356.50

GR662.80 1367.50 $663.801379 .50664 .601393 .30 \quad 665.101404 .50 \quad 666.60$ 1421.00

GR667.20 1434.30 $667.701449 .00 \quad 668.001464 .50 \quad 668.301476 .50 \quad 668.20$ 1488.00

GR668.70 $1503.80 \quad 669.201518 .70 \quad 670.201533 .00 \quad 671.201548 .00672 .60$ 1563.00

GR673.50 1572.50 $675.201593 .00 \quad 677.401611 .50 \quad 678.70 \quad 1622.50 \quad 680.60$ 1639.70

GR683.10 1657.50 685.10 1675.00 686.90 1692.00688 .901709 .00691 .60 1730.80

GR693.30 $1746.70 \quad 695.701773 .00 \quad 700.10 \quad 1799.80 \quad 702.601820 .30 \quad 706.30$ 1849.50

GR709.00 $1873.50 \quad 711.101902 .20 \quad 714.301936 .00 \quad 716.401973 .20 \quad 718.10$ 2009.00

GR721.90 2050.20 717.30 2091.00 719.60 2138.40 726.90 2175.00 729.20 2229.80

GR740.20 2327.50

$\begin{array}{lllllllll}\mathrm{X} 1130.44 & 81 \text {. } 579.0 & 2488.3 & 2700.0 & 1850.0 & 2300.0 & .00000 & .00\end{array}$

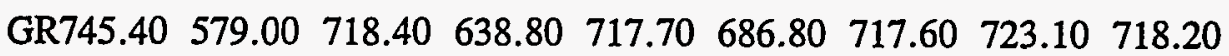
762.70

GR717.80 $803.40 \quad 714.30 \quad 841.80 \quad 706.00 \quad 871.80 \quad 704.40 \quad 900.50 \quad 703.50$ 930.10

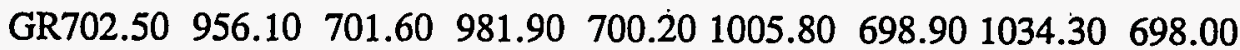
1057.80

GR696.80 1080.40 $695.101105 .90693 .501129 .00 \quad 692.201149 .50 \quad 691.30$ 1170.30

GR691.30 1193.20 690.601217 .00687 .401237 .10685 .601258 .40685 .40 
1276.60

GR685.90 1294.10 686.20 1316.20 685.40 $1340.70 \quad 686.501356 .10 \quad 686.60$ 1377.70

GR684.80 1395.60 684.40 1413.10 684.40 $1435.00 \quad 684.40 \quad 1450.80 \quad 683.40$ 1473.40

GR682.80 1492.50 $682.701511 .70 \quad 681.801531 .50 \quad 681.701547 .90 \quad 681.00$ 1563.90

GR680.80 $1584.00680 .101599 .20 \quad 679.801621 .70 \quad 679.401639 .10 \quad 678.70$ 1657.40

GR678.70 $1681.30 \quad 678.101693 .80 \quad 677.301716 .30 \quad 677.401728 .70 \quad 676.20$ 1746.30

GR676.00 1761.40 $676.001777 .50 \quad 675.801796 .90 \quad 675.201814 .50 \quad 676.30$ 1832.30

GR677.50 1845.90 679.50 1867.70 $680.901885 .60 \quad 682.50 \quad 1904.40 \quad 683.90$ 1927.00

GR685.60 1942.20 687.40 1965.00 688.30 1983.80 690.30 2004.20 691.60 2023.70

GR693.00 2050.30 694.40 2069.80 695.70 2097.10 696.60 2117.10 697.80 2144.00

GR698.50 2164.40 699.40 2184.90 $700.702214 .70 \quad 701.402238 .30 \quad 702.00$ 2267.50

GR703.20 2299.00 703.50 2321.10 706.30 2352.80 712.80 2381.10 723.10 2429.40

GR745.00 2488.30

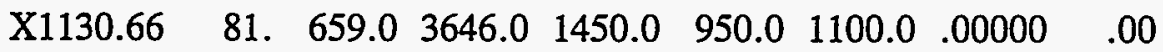

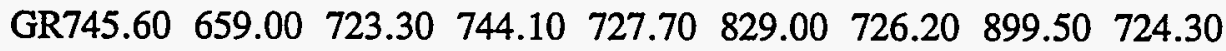
966.00

GR723.50 $1025.30 \quad 723.201091 .00 \quad 722.701152 .70 \quad 722.401210 .00 \quad 722.00$ 1269.40

GR721.80 1332.00 721.50 1390.20 720.70 1444.00 $718.901500 .60 \quad 718.10$ 1554.80

GR717.40 $1603.60716 .001656 .00 \quad 714.301705 .80 \quad 714.301750 .50 \quad 714.20$ 1800.90

GR714.40 $1843.80 \quad 714.501890 .90715 .201936 .00 \quad 716.001987 .80714 .30$ 2036.30

GR707.20 2080.70 706.402118.00 706.102154.80 703.80 2194.00 701.90 2229.60

GR701.20 2259.00 697.002292.50 693.002327.00 688.40 2355.60 683.50 2381.00

GR682.90 2407.80 682.20 2430.00 681.50 2453.00 680.40 2479.00 680.00 2499.00

GR680.60 2528.00 679.20 2556.50 678.20 2576.00 677.40 2601.80 677.00 2623.50

GR675.90 2646.00 675.80 2669.00 675.40 2691.80 $675.802714 .50 \quad 674.60$ 2736.30

GR672.90 2756.50 672.102780.60 671.80 2802.40 671.60 2826.70 671.50 
2845.90

GR671.70 2866.00 $670.602886 .90 \quad 670.002899 .00 \quad 669.902930 .00 \quad 672.80$ 2956.20

GR670.30 2973.00 674.60 2987.80 674.70 3016.30 $675.403035 .50 \quad 676.50$ 3064.00

GR677.70 $3093.00 \quad 678.203110 .40 \quad 680.403136 .80681 .203156 .80682 .60$ 3182.00

GR684.60 3208.00 686.00 3231.60 688.10 3262.00 689.70 3292.80 694.30 3317.50

GR713.50 3366.60 716.50 3399.00 719.40 3452.40 721.20 3507.00 721.20 3579.90

GR745.80 3646.00

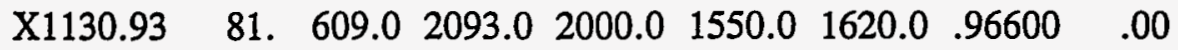

GR761.00 $609.00 \quad 732.40 \quad 675.70 \quad 715.50 \quad 713.80 \quad 702.00 \quad 739.90 \quad 694.30$

761.60

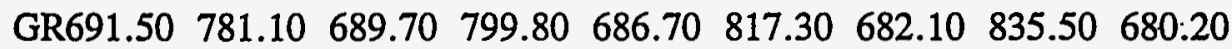
847.80

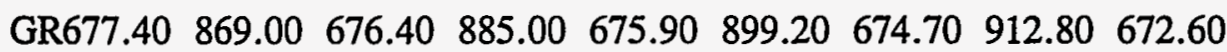
932.70

GR672.10 $946.60 \quad 672.20 \quad 963.00 \quad 671.50 \quad 979.80 \quad 670.00 \quad 992.90 \quad 669.20$ 1012.90

GR668.90 $1023.60668 .401038 .60667 .901051 .60 \quad 667.401065 .40666 .60$ 1081.40

GR666.70 $1098.50 \quad 667.401110 .50 \quad 666.701126 .20 \quad 666.201139 .90 \quad 666.40$ 1154.20

GR667.70 $1168.20668 .601181 .90 \quad 670.601198 .30 \quad 671.201214 .30 \quad 672.60$ 1227.40

GR673.60 1242.10 674.50 $1259.50 \quad 675.301275 .90 \quad 675.601289 .90 \quad 676.40$ 1310.10

GR676.50 1322.90 677.10 1342.90 677.40 1354.90 677.90 1375.40 678.10 1387.60

GR678.20 $1407.60 \quad 678.801420 .80 \quad 679.401442 .40 \quad 679.50 \quad 1453.30 .680 .60$ 1473.30

GR680.80 1486.50 $681.301503 .30 \quad 681.701520 .70 \quad 682.501542 .90 \quad 683.00$ 1555.00

GR683.70 $1576.40 \quad 683.701589 .80684 .301607 .20 \quad 684.301624 .60 \quad 683.40$ 1638.70

GR683.30 $1660.00 \quad 683.701677 .90 \quad 683.001694 .80 \quad 683.301717 .60 \quad 683.80$ 1730.30

GR684.80 $1748.80 \quad 685.001764 .30 \quad 685.401784 .30 \quad 685.801801 .20 \quad 686.50$ 1817.10

GR687.50 $1836.30 \quad 688.201854 .40 \quad 689.901872 .90 \quad 691.40 \quad 1893.40 \quad 693.30$ 1911.60

GR698.20 1935.50 706.20 1954.90 719.30 1986.50 721.90 2011.10 725.10 2044.80

GR745.60 2093.00 
$\begin{array}{lllllllll}\mathrm{X} 1131.62 & 81 . & 695.0 & 3293.0 & 3600.0 & 2750.0 & 3280.0 & .00000 & .00\end{array}$

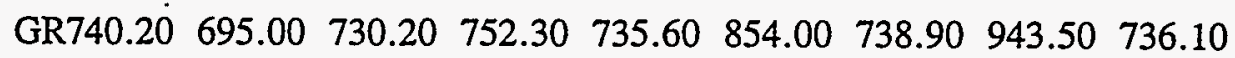
1052.00

GR734.20 1142.30 730.80 1216.50 728.10 1274.30 $725.601333 .00 \quad 724.70$ 1385.40

GR725.10 1438.50 725.70 $1494.00725 .801544 .30 \quad 727.001602 .80 \quad 727.80$ 1655.50

GR728.10 $1716.80725 .801770 .00 \quad 730.601821 .40 \quad 730.301898 .00 \quad 726.90$ 1949.90

GR724.50 2011.00 $721.702061 .00 \quad 719.802104 .30 \quad 719.102139 .90 \quad 699.20$ 2176.50

GR687.90 2198.50 682.00 2220.00 678.30 2238.50 675.70 2257.00 674.20 2272.30

GR671.70 2290.50 670.80 2307.80 671.80 2324.50 673.40 2345.30 674.20 2358.80

GR675.20 2377.00 675.80 2392.50 676.00 2409.30 675.90 2429.30 675.70 2443.00

GR675.60 2464.00 $675.402484 .30 \quad 675.302499 .50 \quad 675.302516 .30 \quad 675.00$ 2534.50

GR675.00 2552.00 675.00 2569.50 674.80 2585.00 $674.702605 .00 \quad 674.90$ 2626.30

GR674.70 2640.00 673.90 2660.30 674.20 2675.00 674.50 2694.70 674.60 2708.80

GR674.70 2729.00 $674.402742 .50 \quad 674.402761 .00 \quad 674.90 \quad 2777.80 \quad 675.30$ 2796.50

GR675.50 2814.50 $676.302836 .30 \quad 676.702849 .80 \quad 677.002869 .00 \quad 677.30$ 2885.00

GR678.00 2904.00 678.902920.50 679.302938.00 680.10 2957.80 680.80 2973.70

GR682.10 2997.00 683.10 3018.00 683.80 3035.50 $684.003054 .50 \quad 686.60$ 3076.40

GR698.20 $3112.50 \quad 697.303121 .30 \quad 700.003149 .40 \quad 714.503179 .00 \quad 725.00$ 3235.50

GR745.50 3293.00

$\begin{array}{lllllllll}\mathrm{X} 1132.05 & 81 . & 499.7 & 2521.4 & 2750.0 & 2350.0 & 2430.0 & : 00000 & .00\end{array}$

GR753.90 $499.70 \quad 746.20 \quad 620.50 \quad 736.50 \quad 707.30 \quad 734.00 \quad 761.20 \quad 733.40$

811.50

GR729.80 $866.00 \quad 729.70 \quad 905.20 \quad 729.60 \quad 942.60 \quad 729.50 \quad 993.40 \quad 729.30$ 1039.90

GR728.80 1081.10 $728.101125 .40 \quad 727.201164 .80 \quad 726.201201 .80 \quad 724.90$ 1243.10

GR723.80 $1279.60722 .501314 .10 \quad 721.401352 .50 \quad 720.401384 .60 \quad 719.30$ 1413.40

GR714.30 $1446.90708 .401472 .80 \quad 704.801498 .70 \quad 700.70 \quad 1524.90 \quad 698.20$ 1543.10 
GR694.20 1565.00 $693.001583 .50 \quad 691.501603 .40 \quad 689.901622 .30 \quad 688.10$ 1644.30

GR686.50 1657.90 684.90 1677.10 684.30 1691.10 683.60 1709.70 682.10 1727.00

GR680.80 $1748.20 \quad 679.80 \quad 1760.10 \quad 678.10 \quad 1779.50 \quad 676.90 \quad 1792.30 \quad 675.50$ 1810.00

GR675.30 1823.40 674.70 1841.60 674.10 1854.10 674.40 $1867.00 \quad 673.40$ 1883.50

GR672.70 1898.40 672.20 1912.70 671.60 1924.00 671.30 1942.60 671.30 1955.90

GR670.50 1971.20 669.50 1986.80 669.10 $1999.60 \quad 668.602013 .10 \quad 667.30$ 2030.00

GR666.00 2044.90 $665.802057 .40 \quad 665.902069 .90 \quad 665.802084 .80 \quad 665.50$ 2100.10

GR666.50 2112.70 $668.002126 .70 \quad 667.402139 .90 \quad 669.902152 .90 \quad 671.50$ 2168.60

GR673.10 2183.80 675.30 2198.90 677.90 2214.70 $679.602230 .80 \quad 680.60$ 2245.60

GR685.90 2263.50 688.00 2282.10 690.50 2300.10 693.90 2318.70 695.50 2340.00

GR703.90 2364.60 707.50 2384.30 709.10 2410.70 719.102439 .60721 .00 2471.90

GR744.00 2521.40

$\begin{array}{lllllllll}\mathrm{X} 1132.71 & 81 . & 568.7 & 1890.8 & 3400.0 & 3550.0 & 3490.0 & .00000 & .00\end{array}$

GR739.50 $568.70 \quad 727.90 \quad 619.50 \quad 724.10 \quad 662.20 \quad 720.90 \quad 693.50 \quad 719.50$.

723.00

GR718.10 .751.00 716.60 779.50.715.30 $807.90 \quad 713.70 \quad 833.20 \quad 712.60$ 858.30

GR711.70 $880.90 \quad 711.00 \quad 903.60 \quad 709.90929 .40 \quad 709.20 \quad 949.10 \quad 707.80$ 973.70

GR706.60 $991.70705 .001015 .30703 .401035 .50 \quad 702.101052 .20700 .80$ 1068.60

GR697.90 $1090.60695 .501108 .30 \quad 690.201122 .10686 .801136 .50 \quad 685.60$ 1151.30

GR684.20 1164.90682 .601179 .40681 .201192 .00679 .601205 .50677 .60 1218.60

GR676.40 1230.90 675.70 1239.20 675.10 1255.10 674.50 1262.30 673.40 1279.60

GR672.50 1292.30 $672.001303 .20671 .301314 .00 \quad 671.201326 .80 \quad 670.90$ 1337.60

GR670.30 1349.40 668.80 1360.30 668.40 1372.10 667.30 1388.30 666.80 1394.20

GR665.80 1409.50 $665.501415 .90 \quad 665.201426 .20 \quad 664.901437 .00 \quad 664.70$ 1443.90

GR663.80 1457.50 $662.701468 .10661 .501478 .90 \quad 660.901489 .20660 .70$ 1499.60 
GR660.00 $1509.40 \quad 659.401520 .20 \quad 659.201526 .60 \quad 659.201539 .10 \quad 658.80$ 1546.80

GR661.30 1558.90 662.901566.00 665.40 1580.50 666.50 1589.60 667.80 1602.10

GR669.10 1613.20 670.50 1624.10 672.30 1634.40 673.30 1647.90 674.90 1656.00

GR678.10 1673.30 $680.401689 .00681 .601697 .90 \quad 685.301712 .10 \quad 689.50$ 1727.10

GR692.20 1736.30 $702.601761 .30 \quad 710.101784 .70 \quad 719.50 \quad 1807.60 \quad 723.60$

1842.30

GR745.00 1890.80

$\begin{array}{lllllllll}\mathrm{X} 1133.41 & 81 . & 608.3 & 2058.7 & 3450.0 & 3850.0 & 3760.0 & .00000 & .00\end{array}$

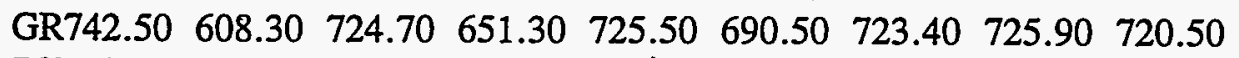

762.70

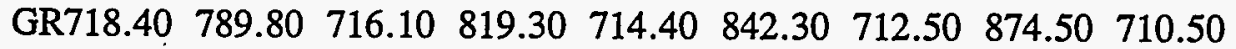

900.10

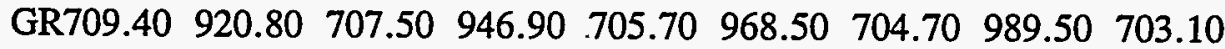

1010.70

GR700.90 $1033.00 \quad 699.201051 .30 \quad 697.801070 .10 \quad 696.501089 .10 \quad 695.00$ 1107.90

GR693.30 1124.60 691.90 $1140.40 \quad 691.301159 .20 \quad 690.101177 .60688 .70$ 1194.90

GR687.10 $1209.10 \quad 686.001226 .50 \quad 684.201241 .40 \quad 683.501257 .40 \quad 682.30$ 1274.30

GR681.70 $1286.40 \quad 680.001301 .10 \quad 679.401316 .20 \quad 678.901328 .40 \quad 676.80$ 1344.90

GR675.40 $1360.80 \quad 674.201371 .70 \quad 673.101386 .30 \quad 672.70 \quad 1398.30 \quad 672.30$

1412.00

GR672.20 1424.90 $672.301437 .70671 .801452 .80 \quad 671.601462 .70 \quad 671.20$ 1478.10

GR669.80 $1491.10670 .101504 .00670 .001519 .90 \quad 670.001530 .40 \quad 669.80$ 1545.10

GR669.50 $1555.80 \quad 669.501569 .10669 .601580 .20 \quad 669.401595 .10 \quad 669.60$ 1607.40

GR669.70 $1619.40 \quad 671.301631 .90 \quad 672.101644 .50 \quad 671.301658 .30 \quad 671.50$ 1670.60

GR671.70 1684.30 673.00 1696.20 671.40 1710.80 672.00 1724.70 672.70 1737.40

GR671.10 $1752.20 \quad 672.001764 .00 \quad 673.30 \quad 1776.40 \quad 675.40 \quad 1791.40 \quad 675.50$ 1806.30

GR675.70 $1818.70 \quad 676.601831 .10 \quad 677.101844 .80 \quad 682.10 \quad 1863.90 \quad 688.50$ 1876.30

GR696.10 $1898.10 \quad 702.001912 .70 \quad 712.901937 .10 \quad 722.80 \quad 1963.90 \quad 725.30$

1999.90

GR750.70 2058.70

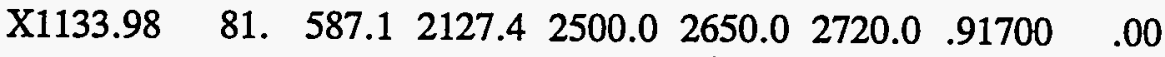




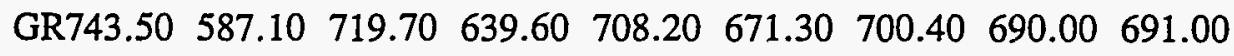
711.50

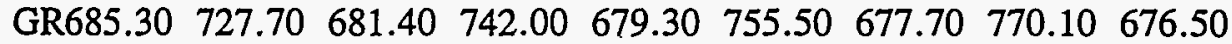
782.70

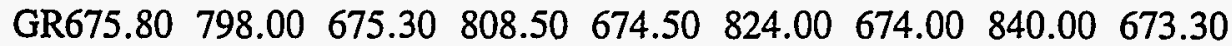
851.00

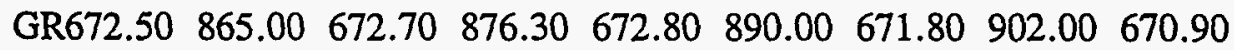
915.00

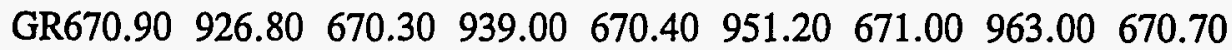
976.00

GR670.30 $988.80 \quad 670.301002 .30 \quad 670.40 \quad 1017.00 \quad 669.60 \quad 1026.80 \quad 668.70$ 1037.50

GR668.70 1051.30 667.90 1064.30 668.20 1076.40 $667.301091 .50 \quad 667.70$ 1099.30

GR668.60 1112.50 $668.601125 .00668 .501136 .50 \quad 668.801148 .50 \quad 669.90$ 1157.50

GR670.30 1174.00 $671.101188 .50 \quad 670.301197 .50 \quad 670.901213 .70 \quad 671.40$ 1224.30

GR672.00 1238.00 $672.201249 .80 \quad 672.301257 .50 \quad 673.201274 .00 \quad 674.20$ 1286.30

GR674.90 1301.10 $675.501313 .00 \quad 676.501327 .00 \quad 677.201341 .00 \quad 678.00$ 1356.30

GR678.00 1373.50 $679.901384 .00 \quad 681.701396 .80 \quad 684.401412 .80 \quad 687.90$ 1431.00

GR688.40 1444.00 $689.601467 .00690 .40 \quad 1477.80 \quad 692.601501 .30 \quad 692.20$ 1511.00

GR696.70 $1527.40 \quad 698.201550 .00 \quad 700.60 \quad 1570.50 \quad 703.10 \quad 1590.50 \quad 709.10$ 1611.30

GR710.10 $1639.00717 .101678 .00718 .101693 .00 \quad 724.501731 .70 \quad 724.40$ 1770.50

GR726.00 $1811.30 \quad 728.501862 .00731 .601884 .80 \quad 733.501976 .10735 .30$ 2051.90

GR738.00 2127.40

$\begin{array}{lllllllll}\mathrm{X} 1134.58 & 81 . & 578.7 & 2516.8 & 2850.0 & 3800.0 & 3130.0 & .00000 & .00\end{array}$

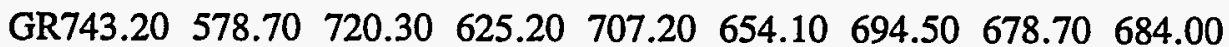
696.30

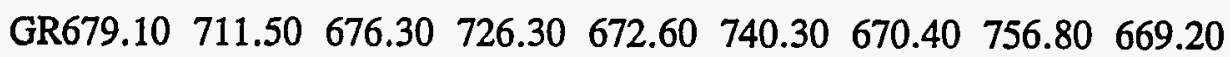
768.30

GR668.90 $785.70 \quad 668.70 \quad 803.60 \quad 668.30 \quad 812.30 \quad 667.80 \quad 826.00 \quad 667.50$ 840.40

GR667.70 $855.30 \quad 670.70 \quad 869.80 \quad 672.50 \quad 883.20 \quad 672.90 \quad 898.40 \quad 673.00$ 912.40

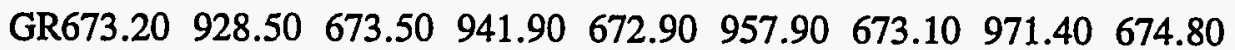
988.20

GR674.90 $1005.60 \quad 677.001019 .30 \quad 678.101033 .40 \quad 679.701050 .40 \quad 680.20$ 1066.80 
GR679.70 $1083.20 \quad 679.901100 .10 \quad 679.80 \quad 1114.00 \quad 679.70 \quad 1129.30 \quad 680.70$ 1146.90

GR681.40 $1164.10683 .301178 .70 \quad 686.601198 .30 \quad 687.701215 .10689 .40$ 1231.80 .

GR692.60 1252.30 $693.701278 .20693 .901291 .70694 .901312 .70 \quad 695.50$ 1329.90

GR696.60 1348.20 697.90 1371.70 $698.301393 .60 \quad 698.501413 .50 \quad 698.20$ 1432.20

GR697.00 1454.40 $695.801478 .20696 .901496 .60 \quad 698.301518 .00698 .70$ 1537.90

GR703.70 $1557.30 \quad 703.701582 .70704 .701603 .60 \quad 707.101631 .40 \quad 711.10$ 1661.60

GR717.20 1686.60 720.60‘1721.00 724.20 $1753.40 \quad 721.201789 .30 \quad 722.70$ $1824.40^{\circ}$

GR723.50 $1857.10 \quad 723.901894 .10724 .601932 .10 \quad 726.101971 .30 \quad 726.50$ 2010.60

$\begin{array}{llllll}\text { GR726.30 2049.40 } 726.302084 .70 & 726.50 & 2126.40 & 727.00 & 2167.10 & 727.70\end{array}$ 2208.60

GR728.00 2251.00 $729.202295 .10 \quad 732.102323 .70 \quad 733.802391 .20 \quad 730.90$

2440.10

GR753.20 2516.80

$\begin{array}{lllllllll}\mathrm{X} 1135.15 & 81 . & 562.5 & 2061.1 & 3000.0 & 2500.0 & 2990.0 & .00000 & .00\end{array}$

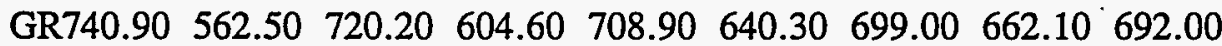

680.50

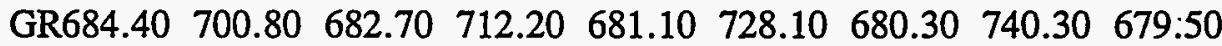

755.70

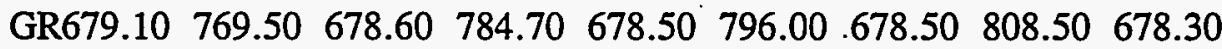

825.30

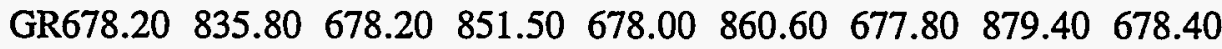

896.30

GR679.70 $906.70 \quad 681.60 \quad 922.70 \quad 682.60 \quad 935.70 \quad 683.40 \quad 947.80 \quad 683.90$

965.70

GR684.80 983.10684 .80995 .00685 .101011 .40685 .401025 .80685 .30

1041.50

GR685.60 1057.10 685.70 1072.20 685.60 1088.30 686.00 $1104.30 \quad 686.20$ 1120.10

GR685.90 $1136.40 \quad 685.901150 .30 \quad 686.201169 .50 \quad 686.301181 .30 \quad 686.70$ $1200: 90$

GR687.40 $1213.00 \quad 687.401226 .60 \quad 687.201244 .50 \quad 687.301260 .90 \quad 687.80$ 1276.30

GR688.50 1297.10 $688.901308 .80 \quad 688.701324 .40 \quad 690.00 \quad 1341.80 \quad 690.20$ 1361.90

GR690.20 $1375.00 \quad 690.701393 .70 \quad 691.301407 .80 \quad 692.201425 .80 \quad 693.10$ 1441.00

GR693.30 $1459.90 \quad 693.301476 .30 \quad 693.501498 .20 \quad 693.801512 .70 \quad 694.70$ 1532.00 
GR695.20 1547.60 696.50 $1569.30 \quad 697.501586 .10 \quad 697.501605 .00 \quad 697.60$ 1623.80

GR697.40 $1642.20700 .101662 .30 \quad 702.701684 .10703 .601703 .70 \quad 704.60$ 1727.80

GR705.60 $1747.60 \quad 706.60 \quad 1770.20 \quad 710.60 \quad 1797.20 \quad 714.001824 .80 \quad 713.70$ 1851.60

GR713.20 $1876.60713 .701909 .30713 .901940 .90 \quad 715.801965 .20 \quad 725.00$ 2007.00

GR742.80 2061.10

$\begin{array}{llllllllll}\mathrm{X} 1135.76 & 81 . & 610.8 & 2102.6 & 3150.0 & 3600.0 & 3400.0 & .00000 & .00\end{array}$

GR742.00 $610.80 \quad 717.50 \quad 656.00 \quad 704.00 \quad 684.80 \quad 695.10 \quad 705.00 \quad 691.10$ 719.30

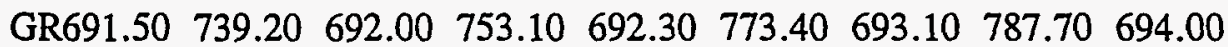
807.90

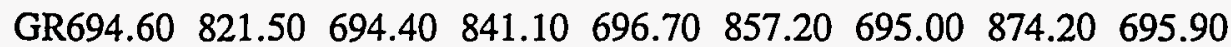
893.00

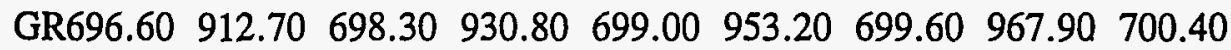
986.40

GR700.70 1006.00 701.30 1025.70 701.50 1045.80 701.90 1066.00 701.60 1086.60

GR701.80 1105.80 701.80 1128.40 702.10 1146.00 701.50 1168.20700 .60 1190.50

GR699.50 1207.10 $698.401224 .00698 .501243 .90 \quad 698.101264 .10 \quad 697.70$ 1283.20

GR696.90 1305.30 $695.901319 .30 \quad 694.501338 .50 \quad 693.201354 .40 \quad 691.70$ 1370.30

GR691.60 1387.00 691.401404 .50690 .701422 .10689 .801438 .00690 .00 1454.40

GR690.30 1469.90 $689.801485 .80 \quad 689.60 \quad 1503.40 \quad 689.50 \quad 1517.40 \quad 689.40$ 1537.20

GR689.40 $1550.90 \quad 689.101566 .40 \quad 689.201581 .40 \quad 689.301594 .30 \quad 689.60$ 1612.50

GR690.00 1626.20 $689.601645 .30 \quad 689.70 \quad 1660.00 \quad 689.201677 .40 \quad 688.90$ 1694.50

GR689.00 $1708.30 \quad 689.101726 .40 \quad 689.201739 .90 \quad 689.501760 .90 \quad 689.80$ 1773.00

GR690.50 1794.00 690.80 1806.80 691.00 1823.80 691.70 1837.70 692.20 1851.10

GR693.90 1871.00693 .001886 .30693 .001906 .90693 .701922 .20695 .10 1942.00

GR696.20 1959.20 $698.801977 .60 \quad 701.601997 .40 \quad 708.702018 .00 \quad 732.20$ 2076.60

GR743.30 2102.60

$\begin{array}{lllllllll}\mathrm{X} 1136.29 & 81 . & 559.6 & 2607.1 & 2950.0 & 2750.0 & 2850.0 & .00000 & .00\end{array}$

GR741.70 $559.60 \quad 717.60 \quad 613.50 \quad 717.20 \quad 655.20 \quad 716.80 \quad 690.50 \quad 717.20$ 
726.20

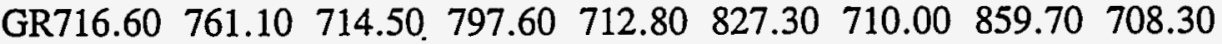
890.20

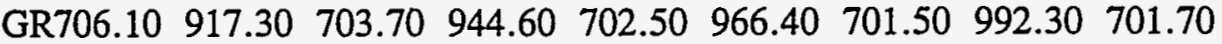
1016.30

GR701.90 $1040.40 \quad 702.201067 .20 \quad 702.601094 .70 \quad 702.701116 .40 \quad 702.30$ 1137.40

GR703.20 1166.50 $700.701192 .50 \quad 699.801213 .60 \quad 698.401235 .40 \quad 697.10$ 1257.80

GR696.10 1279.70 696.00 1301.90 693.60 1323.30 690.70 $1345.10 \quad 690.70$ 1366.80

GR690.50 $1384.40 \quad 689.201405 .70 \quad 687.001422 .60 \quad 685.901439 .20 \quad 685.10$ 1460.30

GR685.60 $1482.20 \quad 686.401494 .80 \quad 686.901513 .60 \quad 688.201532 .10 \quad 687.50$ 1551.40

GR685.50 $1571.00 \quad 684.40 \quad 1590.10 \quad 685.20 \quad 1606.30 \quad 686.301622 .20 \quad 689.40$ 1645.00

GR692.20 1666.20 $698.80 \quad 1684.20 \quad 705.601707 .70 \quad 706.701736 .40 \quad 708.20$ 1765.50

GR709.00 $1791.40709 .601823 .20710 .101853 .20710 .401884 .80 \quad 710.30$ 1912.60

GR710.00 $1938.90709 .401970 .60709 .402002 .60709 .002029 .70 \quad 708.40$ 2057.40

GR708.50 2086.30 707.40 2112.60 $706.302140 .90 \quad 705.102164 .40 \quad 704.50$ 2192.10

GR703.50 2219.60 701.70 2246.10 699.802268.00 696.90 2291.10 694.90 2309.40

GR692.40 2333.80 $691.502351 .60691 .802372 .80 \quad 692.302393 .80 \quad 694.00$ 2416.40

GR696.50 2438.30 $698.602458 .10 \quad 701.502485 .10 \quad 705.102508 .30 \quad 721.50$ 2542.50

GR744.00 2607.10

$\begin{array}{lllllllll}\mathrm{X} 1136.69 & 81 . & 602.2 & 1973.1 & 2500.0 & 1150.0 & 1920.0 & .00000 & .00\end{array}$

GR741.60 $602.20 \quad 724.00 \quad 636.10 \quad 717.00 \quad 677.80 \quad 707.00 \quad 700.10 \quad 705.30$ 719.80

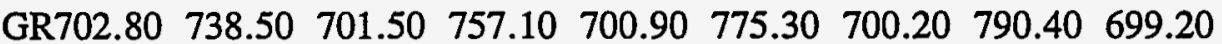

806.80

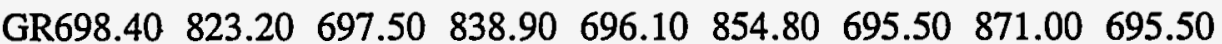
885.60

GR695.30 $901.20 \quad 694.50 \quad 917.20 \quad 694.30 \quad 932.50 \quad 694.60 \quad 946.80 \quad 694.90$ 965.50

GR694.50 $978.70 \quad 693.90 \quad 998.20 \quad 693.30 \quad 1007.80 \quad 692.801023 .00 \quad 692.20$ 1037.30

GR692.00 1054.30 691.80 1067.00 692.10 1083.60 693.40 1096.10 694.20 1109.60

GR695.10 1126.90 $695.301145 .80 \quad 694.901157 .00 \quad 695.401172 .10 \quad 696.20$ 
1188.30

GR695.50 1204.10 695.40 1219.60 695.50 1234.80 $696.101249 .20 \quad 695.70$ 1265.10

GR696.00 1281.30 695.60 1296.90 696.00 1311.80 695.70 1327.10 695.20 1342.20

GR694.90 1358.10 696.10 1373.30 696.70 $1388.10 \quad 696.401406 .30 \quad 696.30$ 1418.70

GR696.00 1437.10 696.20 1455.00 696.30 1467.40 696.00 1485.80 696.70 1500.10

GR696.50 $1515.60696 .501531 .30 \quad 696.901547 .80 \quad 697.40 \quad 1562.80 \quad 697.40$ 1578.80

GR697.60 1594.90 697.70 1610.80 697.40 1626.00 $697.101641 .40 \quad 697.30$ 1659.80

GR697.50 $1676.70 \quad 697.501690 .00 \quad 697.301708 .50 \quad 697.101722 .90 \quad 697.60$ 1739.90

GR697.80 1754.10 698.10 1767.10 698.00 1788.00 697.90 $1807.40 \quad 698.60$ 1820.90

GR700.00 $1839.10700 .801854 .90701 .301874 .90 \quad 706.001888 .90 \quad 724.00$ 1912.40

GR742.50 1973.10

$\begin{array}{lllllllll}\text { ET } & 9.1 & 9.1 & 9.1 & 9.1 & 9.1 & 9.1 & 9.1 & 1440 .\end{array}$

2640.

$\begin{array}{lllllllll}\mathrm{X} 1137.17 & 81 . & 500.0 & 2673.0 & 2600.0 & 2500.0 & 2580.0 & .00000 & .00\end{array}$

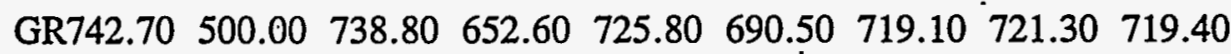
759.00

GR721.50.795.70 $724.30 \quad 831.50 \quad 726.30 \quad 875.30 \quad 725.80 \quad 919.50 \quad 725.40$ 957.60

GR725.80 999.00, 726.50 1051.40726 .801092 .00727 .901136 .70729 .80 1185.30

GR730.60 $1233.50 \quad 729.601289 .00 \quad 728.70 \quad 1335.60 \quad 719.30 \quad 1376.50 \quad 713.90$ 1406.20

GR710.80 1436.00 710.20 $1460.30 \quad 709.20 \quad 1487.80 \quad 707.90 \quad 1508.30 \quad 705.10$ 1537.00

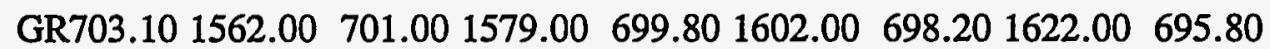
1642.30

GR694.70 $1660.50 \quad 693.801679 .00 \quad 692.701696 .50 \quad 690.801713 .30 \quad 690.30$ 1731.50

GR689.40 1749.30 $689.401766 .50 \quad 689.601784 .00 \quad 688.901802 .00688 .90$ 1820.00

GR689.50 1836.00 690.40 1856.30 691.60 1870.80 692.70 1890.70 692.00 1908.00

GR692.00 1926.70 $691.701941 .80 \quad 691.201962 .00 \quad 690.001977 .30 \quad 687.80$ 1997.00

GR688.90 2011.00 689.80 2031.30 689.30 2046.00 688.70 2067.00 688.20 2080.40

GR686.50 2101.30 $686.802114 .40 \quad 686.102134 .70 \quad 685.502147 .40 \quad 684.90$ 
2163.50

GR684.30 2179.00 683.70 2194.30 683.50 2210.30 685.70 2231.00 688.30 2242.80

GR689.90 2259.50 692.80 2277.00 695.70 2301.00 696.40 2316.00 697.20 2336.30

GR698.30 2354.00 702.50 2375.80 707.60 2398.50 712.40 2423.80 715.60 2454.00

GR714.30 2484.30 717.00 2515.00 716.90 2549.60 716.80 2574.50 724.00 2614.70

GR745.40 2673.00

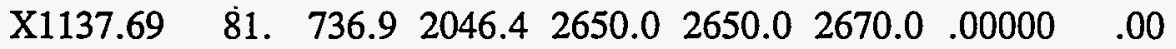

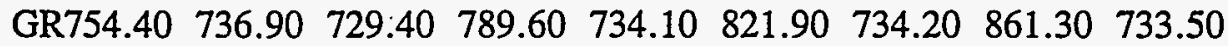

889.50

GR730.40 $934.50 \quad 722.40 \quad 954.40 \quad 720.30 \quad 973.40 \quad 718.60 \quad 998.90 \quad 716.60$

$1017.90^{\circ}$

GR714.90 $1040.90713 .701063 .90 \quad 711.701078 .60 \quad 710.301098 .20 \quad 709.60$

1116.40

GR708.50 $1133.80 \quad 708.001151 .40 \quad 706.901167 .60 \quad 706.101183 .90 \quad 706.10$ 1200.50

GR705.80 1217.30 704.60 1234.80 703.30 1249.80 $700.80 \quad 1269.80 \quad 698.90$ 1282.10

GR697.20 1296.30 $695.301309 .30 \quad 694.401321 .10 \quad 694.801336 .90 \quad 695.10$ 1350.80

GR694.00 1364.80 $693.101379 .70692 .201392 .30 \quad 691.201408 .80692 .00$ 1418.90

GR691.80 1431.70 691.50 1444.50 691.00 1460.10 690.50 1471.30 690.10 1487.30

GR689.90 $1496.80 \quad 689.301509 .70 \quad 688.701521 .90 \quad 688.201531 .70 \quad 687.30$ 1546.90

GR687.00 1560.10 686.90 1572.40 686.70 $1589.70 \quad 686.30 \quad 1597.10 \quad 685.70$ 1612.70

GR685.30 $1621.40684 .701635 .70684 .501644 .40 \quad 684.101658 .10683 .90$ 1668.10

GR683.60 1681.20 683.60 1692.70 683.80 $1704.20 \quad 684.00 \quad 1715.10 \quad 684.20$ 1726.60

GR684.30 1740.10 684.20 $1748.10 \quad 683.701763 .00 \quad 683.801775 .70 \quad 683.10$ 1787.30

GR682.80 $1802.10 \quad 682.501810 .60 \quad 682.10 \quad 1825.10 \quad 682.001833 .10683 .70$ 1845.60

GR684.70 $1856.10688 .201871 .10 \quad 693.001882 .80 \quad 695.501895 .10 \quad 696.70$ 1910.30

GR696.90 1922.10 702.10 1938.30 705.70 $1948.50 \quad 717.301974 .80739 .90$

2015.40

GR745.00 2046.40

$\begin{array}{lllllllll}\mathrm{X} 1137.94 & 81 . & 615.4 & 1979.4 & 900.0 & 1550.0 & 1320.0 & .97200 & .00\end{array}$ 


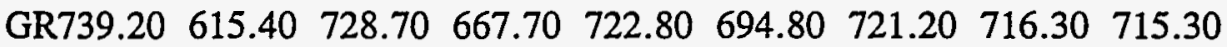
742.80

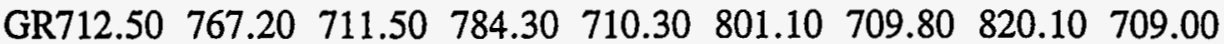
840.20

GR707.80 $858.00 \quad 708.10 \quad 878.90 \quad 707.80 \cdot 892.30 \quad 708.30 \quad 913.80 \quad 708.30$ 928.70

GR708.50 $948.40 \quad 707.80 \quad 966.70 \quad 706.70 \quad 980.70 \quad 707.00 \quad 1000.80 \quad 707.70$ 1014.70

GR707.20 1037.40 706.80 1055.20 706.30 1070.00 705.80 1084.20 706.30 1106.60

GR706.00 $1123.90705 .501140 .20705 .401157 .60 \quad 704.701174 .80 \quad 704.60$ 1191.60

GR703.80 $1205.80703 .601219 .70 \quad 702.701239 .40 \quad 702.101253 .60 \quad 701.60$ 1270.30

GR701.60 1288.20 700.601301 .90698 .701318 .70696 .401331 .10694 .70 1347.60

GR695.90 1359.30 695.00 1375.60 694.20 1387.40 693.20 $1399.80 \quad 692.60$ 1414.40

GR692.10 $1428.00691 .301440 .00689 .701454 .60 \quad 688.501466 .10 \quad 687.30$ 1477.40

GR686.70 $1489.80 \quad 686.80 \quad 1502.10 \quad 686.401514 .50 \quad 685.60 \quad 1526.90 \quad 684.70$ 1538.20

GR683.20 $1549.60681 .80 \quad 1560.90 \quad 680.50 \quad 1572.40 \quad 680.501582 .70 \quad 681.70$ 1595.10

GR684.00 1604.90 $686.401615 .40688 .001628 .00 \quad 690.301639 .10692 .80$ 1655.40

GR695.00 $1673.10696 .401681 .60697 .601694 .90 \quad 700.001710 .90 \quad 701.10$ 1724.60

GR703.80 $1742.40 \quad 706.201760 .80 \quad 707.00 \quad 1776.20 \quad 710.50 \quad 1798.70 \quad 711.80$ 1814.80

GR712.90 $1838.30714 .501855 .10717 .901881 .30 \quad 720.801901 .60 \quad 726.00$ 1926.90

GR746.50 1979.40

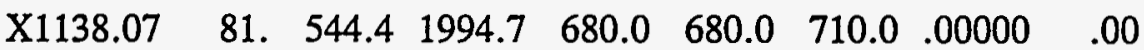

GR737.70 $544.40 \quad 727.20 \quad 591.00 \quad 718.70 \quad 618.80 \quad 713.20 \quad 644.20 \quad 713.00$ 664.90

GR712.30 $684.90 \quad 711.80 \quad 707.80 \quad 711.00 \quad 727.50 \quad 711.00 \quad 749.70 \quad 711.40$ 778.50

GR710.90 $791.80 \quad 710.60 \quad 815.40 \quad 710.70 \quad 832.40 \quad 711.00 \quad 853.90 \quad 711.20$ 873.40

GR710.90 $890.50 \quad 710.90 \quad 916.70 \quad 711.40 \quad 935.50 \quad 711.00 \quad 957.20 \quad 710.90$ 979.90

GR709.40 $998.50 \quad 708.101014 .20701 .801037 .20 \quad 694.601051 .70 \quad 692.90$ 1066.40

GR695.90 $1080.70699 .501096 .60706 .901117 .60706 .901134 .20 \quad 706.60$ 1148.50 
GR707.30 1171.30 706.90 $1191.30 \quad 706.201208 .80 \quad 706.301232 .60 \quad 705.90$ 1246.20

GR705.60 $1268.80705 .601283 .30 \quad 704.501304 .70 \quad 705.001319 .20 \quad 704.40$ 1343.00

GR703.90 $1356.10703 .401379 .20 \quad 703.101390 .90 \quad 702.70 \quad 1406.40 \quad 702.50$ 1423.80

GR701.90 1442.10 701.50 1459.20 $700.801478 .80 \quad 700.001491 .30 \quad 699.60$ 1508.90

GR699.10 1523.20 698.50 1543.40 698.00 1556.60697 .501574 .00696 .90 1587.00

GR696.00 $1603.90694 .901617 .90 \quad 693.301631 .90 \quad 692.40 \quad 1646.90 \quad 691.70$ 1660.30

GR690.80 $1675.00689 .801686 .20688 .301701 .70 \quad 687.00 \quad 1717.00 \quad 686.30$ 1729.40

GR685.10 $1747.60684 .901755 .10684 .801772 .00 \quad 685.001780 .50685 .40$ 1794.40

GR686.30 $1805.90 \quad 686.901815 .90 \quad 686.80 \quad 1832.30 \quad 687.20 \quad 1847.30 \quad 687.20$ 1857.10

GR687.20 $1867.30 \quad 693.801885 .20 \quad 704.101908 .10 \quad 709.901919 .00 \quad 726.30$ 1946.60

GR746.80 1994.70

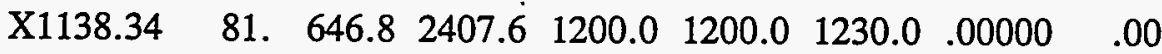

GR762.60 $646.80732 .40 \quad 700.20 \quad 728.10 \quad 739.70 \quad 721.20 \quad 776.10 \quad 715.40$

797.00

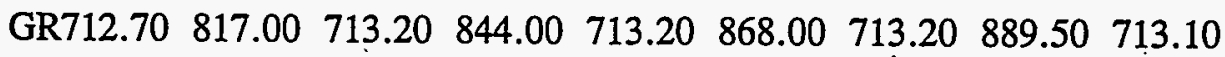
912.50

. GR713.00 934.50 713.20 957.30 $712.70980 .00 \quad 712.101002 .80712 .80$ 1026.00

GR713.00 1048.30 713.30 1074.00 $712.801094 .50 \quad 714.701121 .00 \quad 713.40$ 1140.50

GR712.80 $1166.80713 .601192 .00 \quad 714.401215 .80 \quad 714.501241 .80 \quad 714.30$ 1262.80

GR715.40 1289.00 $714.401311 .50 \quad 713.101334 .80 \quad 714.101357 .00 \quad 716.20$ 1380.50

GR716.10 $1406.80 \quad 716.301427 .30 \quad 715.601454 .00 \quad 715.701478 .20 \quad 715.20$ 1503.00

GR714.10 $1530.00712 .401550 .30 \quad 712.301576 .50 \quad 711.901595 .80 \quad 711.40$ 1619.50

GR708.00 $1641.50 \quad 706.801663 .80 \quad 706.501683 .00 \quad 705.501704 .00 \quad 704.50$ 1724.30

GR703.00 1742.80 701.80 1761.50 701.10 $1782.70 \quad 701.40 \quad 1800.30 \quad 700.60$ 1821.30

GR700.20 1840.00 699.70 1860.30 699.10 1875.30697 .601896 .30696 .60 1913.50

GR696.40 1930.70 696.501947 .50696 .801965 .30696 .501982 .50696 .60 1999.70 
GR696.40 2020.30 696.60 2035.70 696.80 2056.30 697.40 2070.30 696.50 2092.00

GR696.20 2114.70 696.302128.00 695.80 2148.30 695.302163.00 694.60 2184.50

GR693.20 2197.00 692.002213.00 691.102231.00 690.90 2246.00 690.50 2264.00

GR689.90 2282.00 694.10 2295.90 $711.402327 .50 \quad 715.302337 .00 \quad 730.10$ 2366.50

GR746.90 2407.60

$\begin{array}{lllllllll}\mathrm{X} 1138.52 & 81 . & 678.4 & 2660.4 & 1000.0 & 1200.0 & 1160.0 & .00000 & .00\end{array}$

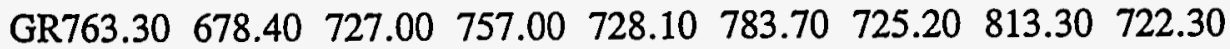

845.70

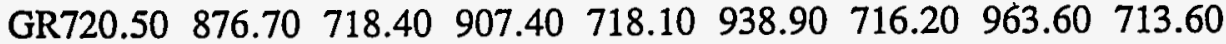

989.20

GR713.50 1015.30 $713.301041 .20712 .301066 .10711 .10 \quad 1090.70 \quad 710.10$

1115.80

GR711.00 1136.20 $711.201165 .60711 .001192 .60 \quad 710.401215 .30 \quad 710.80$ 1237.70

GR710.10 1264.00 709.50 1289.50 710.10 1314.30 710.20 $1338.20 \quad 710.00$ 1360.60

GR710.80 1383.00 $710.601411 .30708 .501437 .70706 .901458 .60 \quad 707.50$ 1481.20

GR707.70 $1503.90 \quad 707.901525 .80707 .001551 .90 \quad 703.501573 .30 \quad 704.60$ 1596.90

GR705.70 $1616.10704 .801639 .90704 .401660 .60708 .501685 .70 \quad 712.90$ 1711.60

GR710.80 $1737.40 \quad 710.301761 .60 \quad 708.901783 .90 \quad 707.601805 .90 \quad 706.20$ 1830.50

GR704.50 $1850.10 \quad 703.401876 .00 \quad 702.00 \quad 1895.10 \quad 700.401918 .30 \quad 699.20$ 1941.90

GR697.30 1960.00 695.10 1981.10 694.50 1997.40 694.20 2019.30 694.80 2037.80

GR695.50 2060.40 695.602074.80 696.70 2093.30 $699.402115 .80 \quad 702.10$ 2139.00

GR703.80 2157.40 $704.402175 .80 \quad 704.402201 .10 \quad 704.302222 .00 \quad 704.20$ 2245.10

GR703.50 2267.30 $706.002290 .40 \quad 709.502312 .10 \quad 709.802337 .70 \quad 710.20$ 2362.40

GR709.70 2387.10 $709.202412 .10709 .602435 .20 \quad 710.102457 .60 \quad 710.50$ 2484.70

GR711.40 2507.30 $710.802533 .20 \quad 712.902562 .00 \quad 721.802587 .40 \quad 726.90$ 2617.90

GR744.00 2660.40

$\begin{array}{lllllllll}\mathrm{X} 1138.71 & 81 . & 764.0 & 2838.5 & 750.0 & 1200.0 & 1060.0 & .00000 & .00\end{array}$

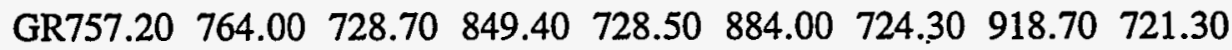


954.50

GR719.60 $989.00 \quad 717.601017 .50 \quad 713.90 \quad 1045.50 \quad 713.801074 .00 \quad 709.30$

.1104 .00

GR709.50 $1128.00 \quad 706.801153 .00 \quad 705.101176 .50 \quad 706.601200 .30 \quad 706.90$ 1225.50

GR707.00 $1249.50 \quad 707.401273 .50 \quad 705.901298 .00 \quad 705.401322 .00706 .10$ 1346.50

GR703.60 1370.00 702.40 1394.80 $703.201415 .80703 .401442 .00 \quad 704.10$ 1461.50

GR704.40 $1484.70 \quad 699.701506 .00700 .001526 .50 \quad 704.201550 .00 \quad 705.70$ 1573.80

GR707.20 $1597.50 \quad 707.801621 .30 \quad 708.101649 .00 \quad 707.601669 .80 \quad 706.40$ 1697.00

GR706.40 $1718.00 \quad 705.40 .1747 .00 \quad 704.601771 .30 \quad 704.301794 .00 \quad 703.50$ 1818.00

GR702.70 $1839.00699 .301859 .80700 .101883 .00 \quad 703.801907 .50 \quad 704.00$ 1929.00

GR704.40 $1955.00704 .501975 .30704 .702003 .50704 .602023 .40 \quad 705.50$ 2045.00

GR705.10 2070.00 705.50 2099.80 705.30 2118.60 705.50 2141.70 706.00 2166.00

GR709.60 2195.50 $712.402216 .80 \quad 714.402244 .00 \quad 710.802269 .50 \quad 707.80$ 2293.80

GR705.00 2320.80 704.10 2341.50 703.80 2367.30 704.20 2387.50 704.20 2414.00

GR701.60 2435.30 699.902460 .00698 .602478 .70697 .802501 .00697 .10 2520.80

GR696.60 2544.00 696.10 2562.30 696.90 2583.30 $700.002604 .30 \quad 706.50$ 2630.00

GR711.90 2649.30 717.20 2680.80 $721.002713 .80 \quad 722.302745 .50 \quad 727.20$ 2780.80

GR746.50 2838.50

$\begin{array}{lllllllll}\mathrm{X} 1138.94 & 81 . & 861.5 & 3130.8 & 950.0 & 1750.0 & 1450.0 & .00000 & .00\end{array}$

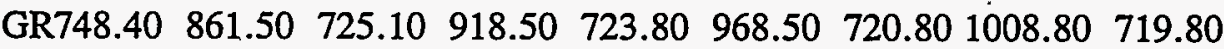
1046.00

GR718.10 1079.50 716.40 1111.50 712.00 1142.50 708.70 1171.00 707.20 1193.80

GR705.80 $1222.80704 .701243 .30704 .801274 .00 \quad 704.601297 .70 \quad 704.80$ 1323.00

GR704.10 1348.00 $702.301370 .50 \quad 701.301392 .50 \quad 700.401417 .00 \quad 698.70$ 1435.70

GR697.40 1460.00 694.50 1484.30 694.50 1500.00 696.90 1521.70 696.10 1544.80

GR695.00 1560.00 693.10 1583.80 693.80 $1604.50 \quad 694.801624 .60697 .00$ 1648.30

GR699.20 1666.50 700.70 1691.80 702.70 $1713.80 \quad 705.501741 .50 \quad 705.50$ 
1763.30

GR704.90 $1790.50 \quad 707.501812 .50708 .201840 .50 \quad 706.101864 .50 \quad 705.00$ 1889.80

GR705.70 $1915.00705 .801939 .00704 .801964 .50 \quad 702.201988 .80 \quad 701.30$ 2013.50

GR701.20 2032.70 701.40 2056.50 702.50 2082.00 702.70 2106.60 703.80 2130.80

GR704.30 2152.00 698.90 2176.50 697.60 2198.00 696.70 2219.30 699.70 2242.50

GR708.10 2264.50 704.70 2289.00 702.20 2313.80 702.50 2339.00 702.20 2363.30

GR702.80 2385.00 $703.002412 .80 \quad 703.802434 .30 \quad 704.302462 .50 \quad 706.10$ 2483.80

GR706.80 2513.50 705.70 2535.30 $705.202563 .00 \quad 707.602585 .50 \quad 713.60$ 2614.80

GR714.00 2640.50 $716.302672 .20 \quad 719.902710 .00 \quad 723.202752 .50 \quad 727.80$ 2788.00

GR731.40 2841.80 730.902893.00 730.002949.50 730.802996 .00731 .80 3057.90

GR747.00 3130.80

$\begin{array}{lllllllll}\mathrm{X} 1139.22 & 81 . & 873.6 & 2983.6 & 1050.0 & 1050.0 & 1050.0 & .00000 & .00\end{array}$

GR740.30 $873.60 \quad 727.40 \quad 935.10 \quad 725.50 \quad 987.70 \quad 723.90 \quad 1032.50 \quad 721.90$ 1075.50

GR719.80 $1113.20 \quad 717.301147 .30 \quad 715.201176 .20 \quad 712.101211 .10 \quad 710.00$ 1238.80

GR707.10 $1264.30 \quad 705.301284 .20 \quad 703.40 \quad 1313.80 \quad 702.20 \quad 1337.10 .700 .10$ 1358.70

GR697.70 1378.90 $696.501399 .30694 .301418 .90 \quad 693.401439 .30693 .60$ 1460.20

GR694.90 $1478.90 \quad 695.50 \quad 1500.30 \quad 695.801517 .80 \quad 697.50 \quad 1541.30 \quad 700.40$ 1561.40

GR703.50 $1588.40 \quad 704.101607 .50 \quad 704.30 \quad 1634.20 \quad 704.50 \quad 1655.20 \quad 704.90$ 1681.60

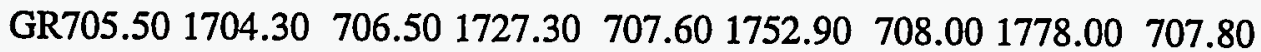
1806.00

GR708.00 1834.70 708.10 1858.90702 .601886 .60701 .801906 .00699 .70 1930.90

GR699.70 1950.90 699.70 1975.30 699.20 1993.80 $699.302014 .70 \quad 699.30$ 2035.90

GR700.10 2056.10 699.60 2079.30 699.80 2101.30 $700.202124 .20 \quad 700.40$ 2147.10

GR700.90 2166.60 701.90 2192.00 703.102213.90 704.50 2236.90 706.10 2258.90

GR708.60 2287.10 709.80 2314.70 711.402343 .90710 .902370 .10710 .80 2402.00

GR711.60 2426.40 $710.502458 .80 \quad 709.602482 .20 \quad 709.002508 .90 \quad 708.20$ 
2533.10

GR707.40 2562.50 706.90 2587.00 706.60 2613.60 706.10 2636.90 705.80 2659.50

GR704.80 2686.20 704.10 2706.40 703.40 2734.10 703.20 2758.00 702.80 2782.00

GR703.10 2804.60 703.30 2827.30 $710.902851 .30 \quad 717.002885 .70 \quad 725.60$ 2934.50

GR744.30 2983.60

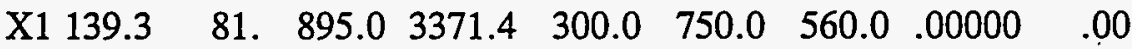

GR739.30 $895.00 \quad 726.80 \quad 959.00 \quad 722.20 \quad 1025.20 \quad 718.40 \quad 1067.20 \quad 714.10$ 1103.60

GR709.90 $1137.60 \quad 706.00 \quad 1170.80 \quad 705.30 \quad 1200.20 \quad 702.80 \quad 1227.90 \quad 701.00$ 1251.90

GR700.50 1278.10 $700.401311 .50 \quad 698.501330 .70 \quad 697.40 \quad 1356.40 \quad 696.90$ 1378.90

GR696.30 1405.50 $695.801427 .80 \quad 696.201452 .00 \quad 697.70 \quad 1474.00 \quad 699.90$ 1500.30

GR702.00 $1523.90 \quad 702.101552 .20 \quad 702.70 \quad 1578.30 .702 .601609 .20 \quad 702.20$ 1633.70 .

GR701.90 1659.60 $701.401683 .90701 .301714 .80 \quad 701.901740 .70 \quad 706.60$

1765.10

GR705.70 $1795.60 \quad 709.601829 .70 \quad 706.301858 .20 \quad 703.601885 .50 \quad 704.10$ 1914.30

GR704.20 $1947.30 \quad 704.50 \quad 1971.20 \quad 706.302002 .10 \quad 708.80 \quad 2028.60 \quad 709.90$ 2062.50

GR710.70 2093.10 711.10 2124.80 $711.402160 .70 \quad 713.302194 .80 \quad 715.50$ 2227.30

GR710.20 2261.60 712.00 2297.80 $710.402329 .30 \quad 709.302359 .90 \quad 710.60$ 2396.30

GR709.60 2428.40 708:90 2455.80 $708.002489 .50 \quad 706.602522 .80 \quad 708.10$ 2551.90

GR708.10 2581.80 708.00 2610.80 708.20 2642.00 707.30 2672.80 708.20 2703.30

GR712.20 2733.90 $714.702767 .40 \quad 706.802803 .40 \quad 707.402833 .80 \quad 705.80$ 2862.60

GR705.80 2888.70 $704.602920 .70 \quad 703.702952 .50 \quad 704.202977 .30 \quad 703.30$ 3001.60

GR701.90 3031.00 $702.403059 .30 \quad 702.803085 .60 \quad 702.303114 .80 \quad 702.90$ 3138.80

GR707.30 $3166.40 \quad 708.403197 .90 \quad 707.603227 .30 \quad 709.803257 .90 \quad 722.50$ 3305.40

GR743.50 3371.40

$\begin{array}{lllllllll}\mathrm{X} 1139.43 & 81 . & 946.6 & 2059.5 & 550.0 & 1100.0 & 910.0 & .00000 & .00\end{array}$

GR736.70 $946.60733 .70 \quad 982.70730 .80 \quad 1018.80 \quad 728.601039 .80 \quad 724.00$ 1071.00 
GR721.10 1089.40717 .701107 .10715 .001119 .20709 .901139 .10706 .80 1154.50

GR705.40 1163.80 704.401170 .00700 .901187 .60699 .701195 .90699 .00 1208.30

GR698.40 1216.30 $697.301229 .20696 .80 \quad 1237.10 \quad 696.001249 .30 \quad 695.40$ 1256.50

GR696.00 1269.30 697.00 1282.40 696.60 1290.70 696.20 1298.90 694.90 1309.40

GR694.10 1318.80 694.30 1329.30 694.10 $1340.60 \quad 694.001348 .80 \quad 693.80$ 1361.10

GR694.00 1367.30 694.50 1378.50 695.10 1387.50 $695.401398 .40 \quad 695.50$ 1407.40

GR696.00 1419.80 $696.601427 .60 \quad 697.201441 .20 \quad 697.901448 .00 \quad 698.90$ 1461.60

GR699.50 1467.80 $700.201480 .60 \quad 700.201489 .20 \quad 700.70 \quad 1502.40 \quad 701.10$ 1512.40

GR701.30 1522.80 701.50 1533.80 $701.501544 .80701 .701555 .60 \quad 701.60$ 1566.10

GR702.00 $1578.60 \quad 702.201586 .00 \quad 703.201602 .00 \quad 703.701616 .40 \quad 704.00$ 1625.90

GR704.60 1635.30 706.00 $1649.70 \quad 707.40 \quad 1665.10 \quad 708.401675 .10 \quad 709.00$ 1685.60

GR711.30 1702.50 714.90 1719.90 $714.901732 .80715 .701746 .30 \quad 716.80$ 1765.20

GR716.70 $1784.70716 .701796 .30716 .401815 .30 \quad 716.701829 .20 \quad 717.40$ 1846.80

GR717.10 $1863.20 \quad 717.00 \quad 1878.60 \quad 717.00 \quad 1896.80 \quad 717.30 \quad 1910.20 \quad 717.10$ 1931.40

GR717.40 1952.00 717.20 1964.50 $716.901984 .40 \quad 717.901999 .00 \quad 728.60$ 2019.60

GR743.30 2059.50

NC $\quad .040 \quad .040 \quad .030$

$\begin{array}{lllllllll}\mathrm{X} 1139.64 & \cdot 81 . & 674.5 & 1641.0 & 1250.0 & 1400.0 & 1340.0 & .00000 & .00\end{array}$

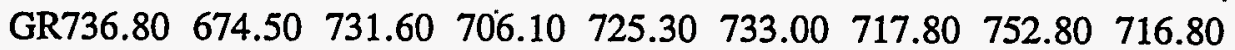
765.20

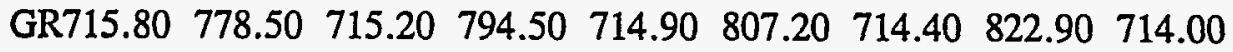
837.30

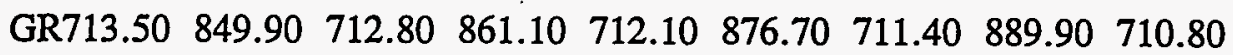
903.80

GR709.60 $917.50 \quad 707.50 \quad 927.40 \quad 705.40 \quad 940.70 \quad 704.60 \quad 949.80 \quad 703.30$ 959.50

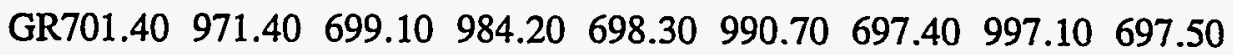
1008.90

GR697.50 1020.80 696.90 1027.50 696.50 1035.60 696.70 1045.70697 .00 1052.50 
GR696.70 1064.30 696.60 1075.20 696.40 1082.10 696.20 1092.50 696.00 1100.50

GR695.70 $1111.80 \quad 695.901118 .50 \quad 696.401129 .60 \quad 696.801137 .20697 .40$ 1149.40

GR697.80 1155.20 698.70 $1168.10 \quad 699.401174 .70 \quad 700.301186 .00 \quad 700.90$ 1193.40

GR701.30 1201.30 702.30 1214.90 $702.901223 .10703 .601235 .40 \quad 704.30$ 1247.80

GR704.70 $1256.20 \quad 705.501271 .00 \quad 705.801278 .40 \quad 706.301294 .80 \quad 706.30$ 1301.40

GR706.60 1317.90 707.00 1324.60 707.40 $1338.30 \quad 707.301346 .90 \quad 707.40$ 1359.10

GR707.50 1370.20 707.70 1384.80 707.80 $1393.90 \quad 707.901409 .90 \quad 707.70$ 1417.10

GR708.00 $1433.70708 .301440 .60708 .701456 .90 \quad 708.501464 .30708 .70$ 1480.10

GR708.50 1488.00 709.00 1503.40 709.00 1512.00 709.30 1526.10 709.50 1536.20

GR709.30 1547.90 709.70 $1560.30712 .801577 .30 \quad 718.701588 .30 \quad 727.90$ 1608.90

GR743.20 1641.00

$\begin{array}{lllllllll}\mathrm{X} 1139.91 & 81 . & 596.6 & 1519.0 & 1300.0 & 1250.0 & 1270.0 & .00000 & .00\end{array}$

GR748.10 $596.60 \quad 740.00 \quad 680.80 \quad 736.40 \quad 695.90 \quad 732.00 \quad 720.00 \quad 724.20$

738.70

GR716.90 $752.00 \quad 714.80 \quad 766.70 \quad 714.40 \quad 776.60 \quad 713.90 \quad 790.90 \quad 714.40$ 798.60

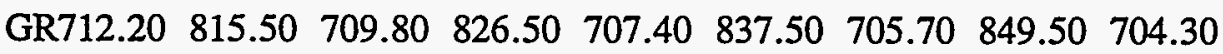
857.00

GR703.40 $862.90 \quad 701.90 \quad 877.40 \quad 701.80 \quad 890.40 \quad 701.40 \quad 895.40 \quad 701.00$ 904.30

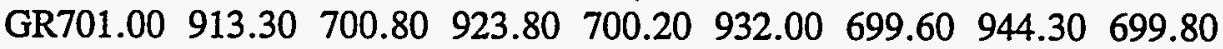
950.50

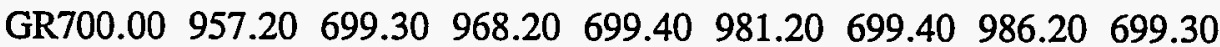
995.70

GR699.201004.20 699.301011.10 699.00 1021.50 698.801028.10 699.00 1039.10

GR699.40 1048.00 699.80 1057.00 700.20 1066.50 700.60 1075.10 701.40 1087.40

GR701.70 $1094.00702 .001106 .90702 .401112 .60 \quad 702.201122 .90 \quad 702.50$ 1130.30

GR702.70 $1138.80702 .801150 .30 \quad 702.701160 .80 \quad 702.601169 .20 \quad 702.80$ 1179.80 .

GR702.90 $1187.40 \quad 702.801201 .20 \quad 703.201207 .30 \quad 703.401216 .70 \quad 703.20$ 1226.60

GR703.30 $1236.60703 .401245 .00 \quad 703.401251 .10 \quad 703.801264 .90 \quad 703.80$ 1272.60 
GR703.70 $1284.00703 .801295 .00704 .201303 .00704 .501315 .90 \quad 704.60$ 1323.90

GR705.00 $1336.90 \quad 705.301343 .70 \quad 705.801357 .40 \quad 705.801363 .80 \quad 705.90$ 1374.30

GR706.10 1384.30 $706.501396 .30 \quad 706.701403 .80 \quad 706.901413 .70 \quad 706.80$ 1424.20

GR705.80 1433.70 $708.201444 .90 \quad 716.201459 .80 \quad 720.801470 .60 \quad 728.40$ 1483.30

GR744.10 1519.00

$\begin{array}{lllllllll}\mathrm{X} 1140.22 & 73 & 571.2 & 1378.2 & 1850.0 & 2000.0 & 1970.0 & .00000 & .00\end{array}$

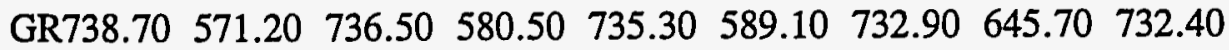
649.80

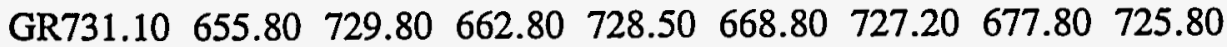
684.70

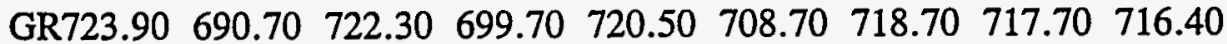

727.60

GR714.50 $738.60 \quad 712.60 \quad 747.60 \quad 711.10 \quad 758.60 \quad 710.00 \quad 769.50 \quad 709.10$

781.50

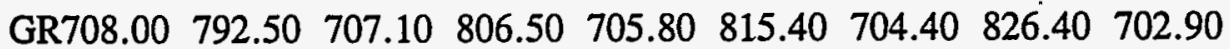
837.40

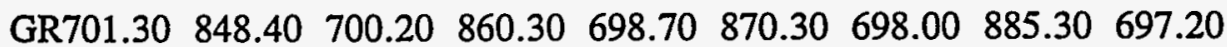
896.20

GR696.50 $909.20 \quad 695.00 \quad 923.20 \quad 693.90 \quad 935.20 \quad 693.20 \quad 948.10 \quad 691.80$ 959.10

GR690.50 $970.10 \quad 689.10 \quad 980.10 \quad 688.10 \quad 991.00 \quad 687.501003 .00 \quad 686.60$ 1014.00

GR686.10 $1025.90685 .501037 .90684 .901050 .90 \quad 684.201063 .90 \quad 683.70$ 1076.80

GR683.40 $1088.80 \quad 683.201100 .80 \quad 682.501112 .70 \quad 682.401125 .70 \quad 682.40$ 1137.70

GR682.60 1148.70 $682.201158 .60 \quad 681.501167 .60 \quad 680.501176 .60 \quad 680.50$ 1183.60

GR680.50 $1192.60 \quad 681.501217 .50 \quad 685.101231 .50 \quad 689.001242 .40 \quad 692.10$ 1254.40

GR694.70 $1266.40 \quad 697.001277 .40 \quad 701.301286 .30 \quad 704.801295 .30 \quad 707.90$ 1303.30

GR721.70 $1332.20 \quad 724.70 \quad 1337.20 \quad 727.50 \quad 1342.20 \quad 732.10 \quad 1348.90 \quad 735.20$ 1354.30

GR743.20 1370.40 744.901376 .90744 .801378 .20

$\begin{array}{lllllllll}\mathrm{X} 1140.51 & 78 . & 591.7 & 1415.5 & 1200.0 & 1400.0 & 1330.0 & .00000 & .00\end{array}$.

GR738.80 $591.70 \quad 736.50 \quad 600.00 \quad 735.40 \quad 607.00 \quad 732.70 \quad 618.40 \quad 732.00$ 622.40

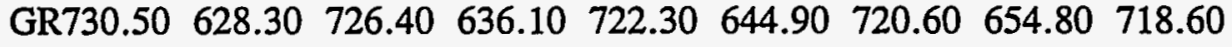
665.60

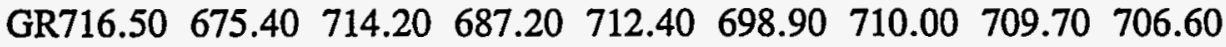


721.50

GR704.60 $731.30 \quad 702.90 \quad 743.10 \quad 701.10 \quad 753.90 \quad 699.80 \quad 765.70 \quad 699.10$ 776.50

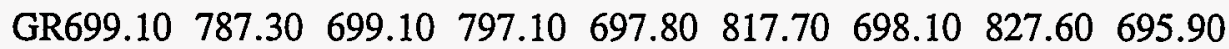
840.30

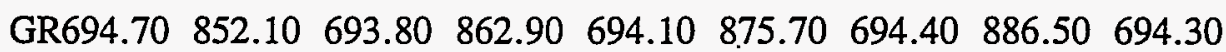
898.20

$\begin{array}{lllllll}\text { GR693.30 } 909.00 \quad 693.20 & 918.90 & 693.50 & 928.70 & 694.20 & 938.50 & 693.70\end{array}$ 950.30

GR693.90 $962.10 \quad 693.70 \quad 972.90 \quad 694.20 \quad 982.70 \quad 694.60 \quad 993.50 \quad 694.60$ 1004.30

GR694.60 1014.10 694.80 1023.90 694.80 1038.60 $694.601051 .40 \quad 694.50$ 1062.20

GR694.40 1072.00 694.401081.80 694.70 1094.60 694.70 1107.40 694.60 1117.20

GR694.60 1129.00 695.00 1139.80 698.70 1152.50 699.50 1161.40 702.70 1172.20

GR706.30 $1181.00 \quad 709.201195 .70 \quad 711.801205 .50 \quad 714.80 \quad 1219.30 \quad 716.20$ 1232.10

GR716.00 1245.80715 .901258 .60715 .601269 .40715 .601279 .20715 .10 1291.00

GR715.10 $1301.80 \quad 715.201311 .60 \quad 715.301321 .40 \cdot 715.801330 .20 \quad 716.30$ 1338.90

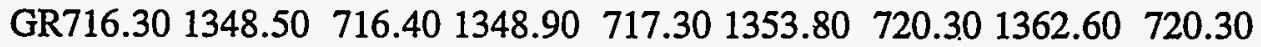
1362.70

GR731.70 1386.60735 .301394 .30743 .801415 .50

$\begin{array}{lllllllll}\mathrm{X} 1140.75 & 81 . & 689.5 & 1918.7 & 1400.0 & 1250.0 & 1350.0 & .00000 & .00\end{array}$

GR735.50 $689.50 \quad 724.60 \quad 713.20 \quad 720.80 \quad 739.80 \quad 718.40 \quad 759.20 \quad 717.20$

777.90

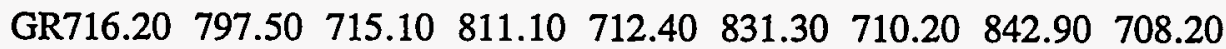

859.40

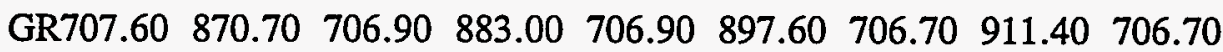

924.50

GR705.70 $941.20 \quad 705.10 \quad 949.30 \quad 705.20 \quad 963.30 .705 .40 \quad 975.10 \quad 705.40$

986.50

GR704.80 1000.80 704.90 1014.90 705.60 1027.90 705.20 1041.10 705.50 1052.90

GR705.70 $1064.80706 .001079 .60706 .401088 .40 \quad 706.201105 .10 \quad 706.00$ 1117.30

GR706.00 $1132.70 \quad 705.701145 .00 \quad 705.801156 .90 \quad 705.901172 .80 \quad 705.10$ 1184.30

GR705.70 $1199.20 \quad 706.201210 .50 \quad 706.801222 .40 \quad 707.301237 .10 \quad 707.60$ 1247.00

GR708.20 $1264.70709 .301283 .40 \quad 710.701292 .30711 .201306 .10 \quad 710.70$

1322.80

GR712.00 1339.10 711.20 1351.90 711.10 1365.20 709.80 1380.40 709.90 
1395.70

GR710.30 $1409.40 \quad 710.301423 .90 \quad 710.901439 .50711 .301457 .10 \quad 712.00$ 1469.20

GR712.10 $1486.80 \quad 711.301499 .30 \quad 710.601514 .90 \quad 710.501528 .40 \quad 710.60$ 1542.40

GR710.50 1559.20 711.00 1575.40 710.60 $1589.00 \quad 710.801603 .50 \quad 710.10$ 1618.00

GR710.30 1632.10 $710.901646 .10 \quad 712.201660 .00 \quad 714.501676 .90 \quad 715.60$ 1694.10

GR715.90 $1711.40715 .301729 .30715 .401744 .40 \quad 715.701765 .70715 .30$ 1779.30

GR715.20 $1796.10 \quad 715.701813 .30 \quad 718.401836 .70 \quad 722.501850 .30 \quad 730.80$ 1878.90

GR743.80 1918.70

$\begin{array}{lllllllll}\mathrm{X} 1141,21 & 81 . & 578.8 & 2023.4 & 2550.0 & 2450.0 & 2550.0 & .00000 & .00\end{array}$

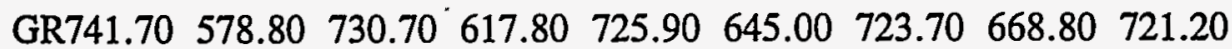
690.80

GR717.70 $709.70 \quad 716.90 \quad 732.00 \quad 713.50 \quad 749.50 \quad 712.70 \quad 768.00 \quad 709.80$ 789.00

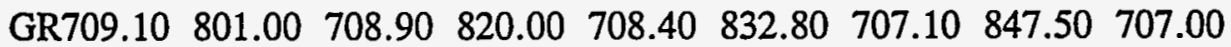
863.00

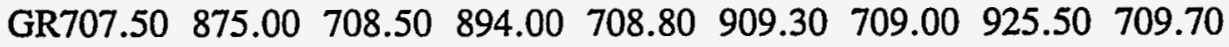

943.00

GR709.70 $958.50 \quad 710.00 \quad 975.70 \quad 709.40 \quad 989.80 \quad 709.90 \quad 1009.30 \quad 710.50$ 1022.80

GR711.70 $1042.30 \quad 712.301055 .50 \quad 712.701073 .30 \quad 712.201092 .30 \quad 712.60$ 1107.30

GR713.20 $1125.00 \quad 713.301142 .00 \quad 713.40 \quad 1161.30 \quad 713.701180 .30 \quad 713.10$ 1196.50

$\begin{array}{llllllll}\text { GR712.50 } 1217.70 & 712.50 & 1231.00 & 712.40 & 1250.30 & 712.30 & 1266.00 & 712.30\end{array}$ 1282.00

GR712.20 1298.50 711.80 1314.30 712.10 $1333.30 \quad 712.301347 .00 \quad 713.00$ 1369.00

GR713.10 $1389.30 \quad 713.001402 .80 \quad 713.00 \quad 1422.00 \quad 712.801438 .50 \quad 712.70$ 1455.70

GR713.00 $1474.00713 .101492 .50712 .501509 .00712 .901529 .70 \quad 713.20$ 1543.30

GR713.70 $1561.70 \quad 713.501578 .50 \quad 713.101594 .70714 .301614 .00 \quad 714.50$ 1628.00

GR711.70 1648.30 709.801661 .00709 .101680 .30708 .901693 .70708 .90 1713.00

GR708.60 1727.30 $708.601744 .50 \quad 709.001761 .30 \quad 709.401778 .00 \quad 709.70$ 1794.00

GR710.30 $1810.00711 .101826 .00711 .701843 .00712 .401861 .00 \quad 713.00$ 1876.00

GR713.60 $1897.00715 .201911 .80719 .501936 .30723 .701953 .80 \quad 728.10$ 
1979.10

GR750.90 2023.40

$\begin{array}{llllllllll}\mathrm{X} 1141.78 & 81 . & 798.7 & 2023.8 & 2450.0 & 2850.0 & 2630.0 & .00000 & .00\end{array}$

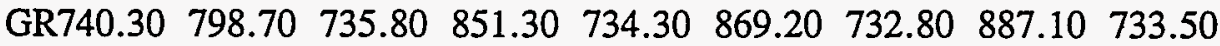

924.00

GR733.90 $945.40 \quad 735.70 \quad 981.30 \quad 736.10 \quad 994.70 \quad 734.80 \quad 1042.30 \quad 734.00$ 1071.70

GR733.20 $1101.10730 .401124 .00727 .801148 .00 \quad 726.501165 .00 \quad 724.20$ 1186.80

GR724.60 $1195.50 \quad 718.10 \quad 1222.10 \quad 715.301236 .00 \quad 713.301250 .50 \quad 711.80$ 1261.00

GR710.40 1274.30 710.40 1282.50 709.60 1300.00 708.90 1315.50 708.90 1323.50

GR709.20 1336.00 708.30 1346.50 706.90 1357.00 706.20 $1371.00 \quad 706.00$ 1379.50

GR705.90 1392.50 705.80 1400.00 705.90 1414.30 705.70 1422.00706 .60 1437.30

GR706.70 $1444.50706 .101459 .00705 .901466 .50 \quad 707.101483 .00 \quad 707.00$ 1494.00

GR706.50 $1506.00706 .401517 .00 \quad 706.801528 .00 \quad 707.301539 .00 \quad 707.30$ 1551.00

GR707.40 $1567.00 \quad 707.601574 .40 \quad 707.501589 .50 \quad 707.601597 .50 \quad 708.00$ 1611.00

GR707.90 $1620.40 \quad 707.801631 .50 \quad 708.201643 .20 \quad 708.201653 .50 \quad 708.20$ 1667.30

GR708.30 $1676.00708 .701690 .40 \quad 708.801701 .30 \quad 709.001714 .10 \quad 709.40$ 1727.00

GR709.30 $1738.50 \quad 709.201749 .50 \quad 708.701763 .30 \quad 708.30 \quad 1772.00 \quad 707.60$ 1785.50

GR707.30 $1793.50707 .101808 .00 \quad 707.001815 .50 \quad 707.401832 .00 \quad 707.50$ 1844.00

GR707.90 $1855.00710 .701873 .00 \quad 712.001880 .40 \quad 713.201895 .50 \quad 713.60$ 1906.50

GR714.10 $1917.00 \quad 715.101932 .10 \quad 718.701949 .80 \quad 724.101964 .30 \quad 733.00$ $1990: 50$

GR747.20 2023.80

$\begin{array}{lllllllll}\mathrm{X} 1142.40 & 87 . & 950.0 & 1947.8 & 3900.0 & 3750.0 & 3810.0 & .00000 & .00\end{array}$

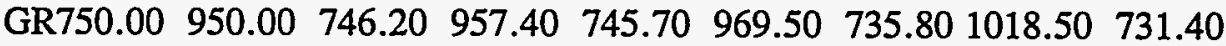
1030.60

GR730.40 $1035.60728 .301041 .50 \quad 725.901047 .50 \quad 724.501055 .50 \quad 724.00$ 1063.40

GR724.10 $1073.40723 .401083 .30 \quad 723.401094 .30 \quad 723.501102 .00 \quad 722.90$ 1112.10

GR722.90 1142.40 $722.401152 .50 \quad 722.401179 .40 \quad 722.101186 .10 \quad 722.60$ 1194.50

GR722.40 1206.30721 .901214 .70722 .001216 .70722 .101226 .60721 .60 


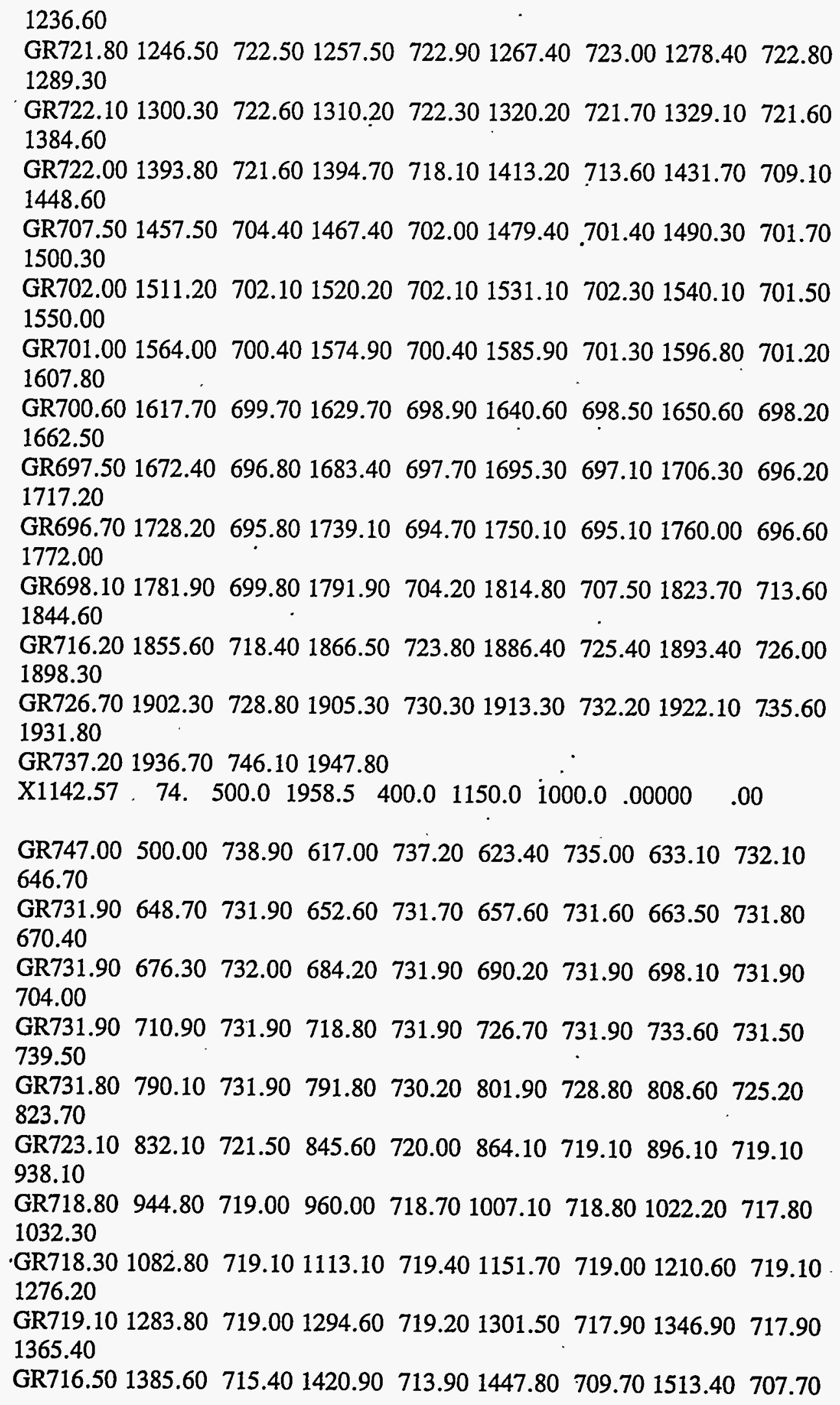


1538.60

GR706.40 1574.00 706.60 1629.50 706.00 $1674.90 \quad 705.401711 .90 \quad 705.80$ 1722.30

GR706.50 1733.10 706.10 $1747.00 \quad 708.30 \quad 1759.80 \quad 707.901772 .60 \quad 708.80$ 1782.50

GR709.80 $1792.40 \quad 710.901803 .30 \quad 712.301812 .20 \quad 714.101821 .00 \quad 716.00$ 1827.00

GR716.50 $1831.90 \quad 717.601832 .90 \quad 720.40 \quad 1840.80 \quad 723.401846 .70 \quad 732.20$

1866.10

GR734.90 1872.90 743.60 1889.10 $743.801948 .90 \quad 748.901958 .50$

$\begin{array}{lllllllllll}\mathrm{X} 1143.14 & 83 . & 611.1 & 1438.8 & 2800.0 & 2550.0 & 2680.0 & .00000 & .00\end{array}$

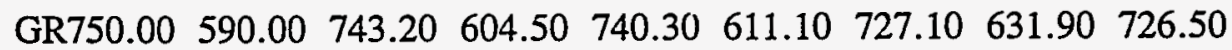

644.00

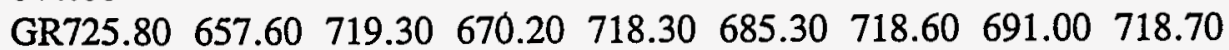

701.30

GR717.20 710.50 715.20 723.10 713.70 $730.00 \quad 712.00 \quad 738.50 \quad 711.20$

747.80

$\begin{array}{lllllll}\text { GR710.40 } 758.30 \quad 709.60 & 765.10 & 708.50 & 774.70 & 708.10 & 780.30 & 707.30\end{array}$

789.50

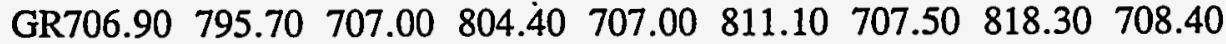

827.00

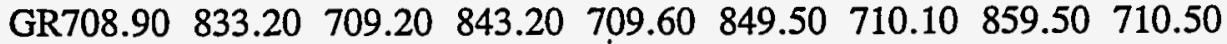

869.80

GR710.90 $876.30 \quad 711.30 \quad 886.70 \quad 711.70 \quad 893.60 \quad 711.90 \quad 902.60 \quad 712.00$

911.30

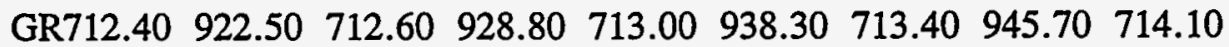

953.20

GR714.50 $964.40 \quad 714.60 \quad 973.00 \quad 714.90 \quad 982.50 \quad 715.20 \quad 994.80 \quad 715.30$

1002.50

GR715.40 $1010.70 \quad 715.501021 .60715 .701031 .50 \quad 716.001039 .70 \quad 716.20$ 1047.90

GR716.20 1059.40 $716.101070 .30716 .301079 .30 \quad 716.601091 .60 \quad 716.60$

1098.50

GR716.70 $1108.90 \quad 716.901117 .80 \quad 717.101127 .30 \quad 717.301138 .50 \quad 717.40$

1146.60

GR717.60 1158.50 $718.201171 .40 \quad 718.501178 .10 \quad 718.901190 .30 \quad 719.20$

1200.20

GR719.60 $1209.10 \quad 719.901220 .80 \quad 720.101228 .90 \quad 720.401242 .40 \quad 720.50$

1253.30

GR720.80 $1265.60 \quad 721.101277 .60 \quad 721.301286 .50 \quad 721.601301 .90 \quad 721.50$

1309.70

GR721.50 1324.10 $722.001333 .60 \quad 724.001347 .40 \quad 726.70 \quad 1358.40 \quad 730.00$

1375.80

GR733.00 1389.30 738.10 1419.40. 746.601438 .80 
$\begin{array}{lllllllll}\mathrm{X} 1143.31 & 81 . & 620.4 & 1610.6 & 1200.0 & 1300.0 & 1260.0 & .00000 & .00\end{array}$

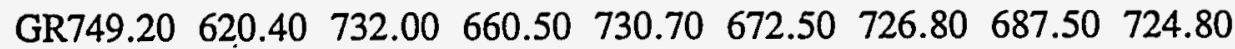
705.00

$\begin{array}{lllllll}\text { GR723.50 } 721.50 \quad 723.20 & 732.30 & 723.10 & 744.00 & 722.20 & 758.00 & 720.20\end{array}$ 766.50

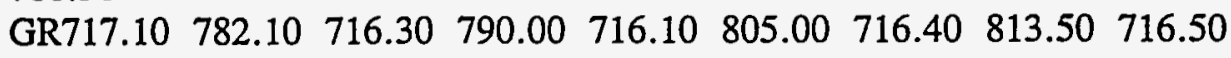
827.30

$\begin{array}{lllllll}\text { GR716.10 } 836.00 & 716.00 \quad 848.80 & 715.80 & 860.00 & 715.50 & 872.00 & 715.60\end{array}$ 883.50

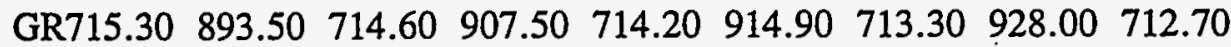
934.80

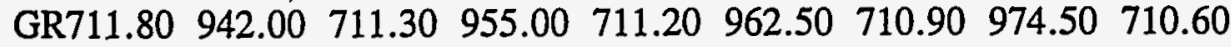
987.00

GR710.60 $994.40 \quad 710.601004 .00 \quad 710.301013 .00 \quad 710.201022 .00 \quad 710.30$ 1031.80

GR710.30 1044.00 710.20 1051.90. 710.401065 .00710 .501070 .50710 .70 1080.00

GR710.80 1090.00 710.80 1102.00 710.90 1109.40 711.20 1119.00 711.30 1129.00

GR711.20 1139.00 711.50 1147.40 712.00 $1155.00 \quad 712.201167 .40 \quad 712.20$ 1178.00

GR712.90 1187.30 $713.301193 .50 \quad 713.701208 .10 \quad 713.701214 .50 \quad 713.90$ 1229.10

GR713.90 $1236.00 \quad 714.201250 .30 \quad 714.50 \quad 1257.00 \quad 714.901271 .00 \quad 715.40$ 1279.00

GR716.10 $1293.00 \quad 716.701301 .00 \quad 717.301315 .00 \quad 717.501323 .50 \quad 718.20$ 1339.00

GR719.20 $1356.00 \quad 719.201362 .30 \quad 719.901376 .00 \quad 720.401386 .00 \quad 722.20$ 1400.30

GR723.30 $1413.00 \quad 723.301428 .80 \quad 725.201441 .00 \quad 728.501455 .80 \quad 730.80$ 1471.60

GR732.00 1490.80 733.20 1509.90 733.60 1517.00 734.70 $1551.80 \quad 741.30$ 1596.00

GR748.00 1610.60

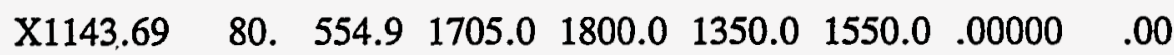

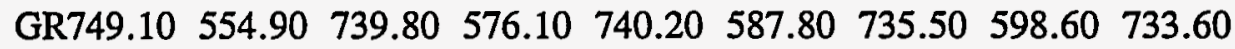
604.20

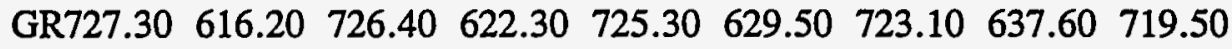
640.50

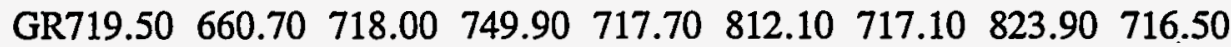
894.50

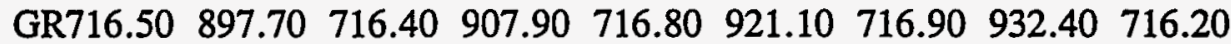
940.50

GR716.10 $952.80 \quad 716.50 \quad 965.00 \quad 715.60 \quad 975.20 \quad 714.90 \quad 985.40 \quad 714.60$ 
997.60

GR714.70 $1008.90 \quad 715.101020 .10 \quad 715.301031 .30 \quad 715.101043 .50 \quad 715.90$ 1054.80

GR716.50 1063.90 716.70 1072.10 $718.201094 .50 \quad 718.601105 .70 \quad 719.30$ 1119.00

GR715.10 $1131.20714 .401142 .50 \quad 715.201152 .70 \quad 715.901165 .90 \quad 718.90$ 1173.10

GR719.70 $1184.30 \quad 714.701195 .50 \quad 714.701207 .70 \quad 714.501219 .00 \quad 714.40$ 1231.20

GR716.10 $1243.40 \quad 717.901254 .70 \quad 719.501266 .90 \quad 720.50 \quad 1278.10 \quad 721.10$ 1288.30

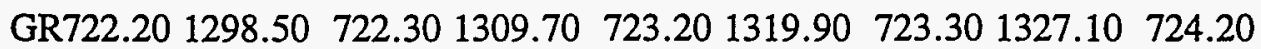
1334.20

$\begin{array}{llllllll}\text { GR724.60 } 1341.30 & 724.10 & 1348.50 & 725.20 & 1353.80 & 726.30 & 1358.80 & 727.50\end{array}$ 1362.20

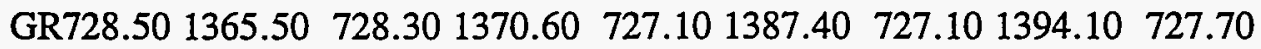
1402.60

GR727.90 $1409.30728 .601416 .00 \quad 728.601419 .40 \quad 729.301424 .40 \quad 730.10$ 1429.50

GR730.10 $1436.20730 .801448 .00730 .801453 .00 \quad 732.601469 .00 \quad 735.30$ 1488.40

GR740.50 1523.00. 741.20 1579.10 741.50 $1632.80 \quad 743.201699 .80 \quad 746.90$ 1705.40

$\begin{array}{llllllllll}\mathrm{X} 1144.73 & 83 . & 551.3 & 1604.7 & 5500.0 & 5100.0 & 5330.0 & .00000 & .00\end{array}$

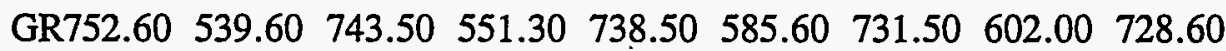
618.90

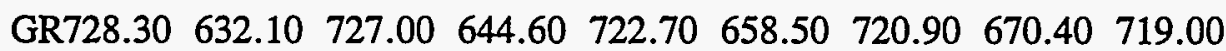
681.00

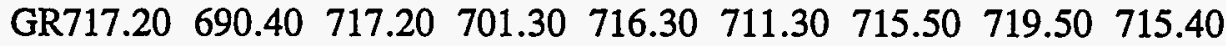

727.70

GR715.30 $738.70 \quad 715.10 \quad 749.10 \quad 715.20 \quad 758.20 \quad 715.10 \quad 765.10 \quad 715.30$

777.00

GR715.40 $788.00 \quad 715.50 \quad 796.20 \quad 715.50 \quad 804.40 \quad 715.40 \quad 815.30 \quad 715.30$

826.30

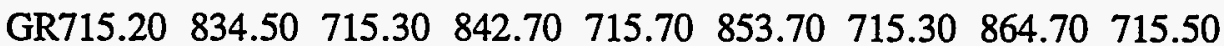

871.40

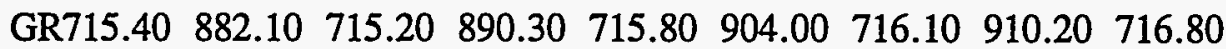

921.40

GR717.30 $929.70 \quad 718.00 \quad 938.30 \quad 718.80 \quad 949.80 \quad 719.70 \quad 961.30 \quad 720.50$

971.70

GR721.40 $985.20721 .80 \quad 994.10722 .40 \quad 1009.00 \quad 723.201018 .00 \quad 723.90$ 1032.50

GR724.40 $1041.00 \quad 724.901055 .90 \quad 725.301067 .40 \quad 725.701078 .80 \quad 726.00$ 1092.20

GR726.20 $1106.30 \quad 726.301120 .00 \quad 726.801135 .60 \quad 727.00 \quad 1147.50 \quad 727.10$ 1159.40 
GR727.40 $1173.70 \quad 727.601188 .30 \quad 727.901203 .00 \quad 728.20 \quad 1217.20 \quad 728.40$ 1231.40

GR728.60 $1244.80728 .601258 .60 \quad 729.201277 .60 \quad 729.101290 .30 \quad 729.50$ 1308.50

GR729.60 1319.60 $730.401337 .70730 .901349 .90 \quad 731.101366 .30 \quad 731.00$ 1382.30

GR731.20 1397.00 730.80 1416.20 730.50 $1432.10 \quad 730.101447 .60 \quad 730.00$ 1465.20

GR729.60 1477.70 $729.601494 .00730 .00 \quad 1509.00^{\circ} 732.701525 .40736 .60$ 1544.50

GR740.90 $1574.10748 .401604 .70 \quad 754.901609 .50$

$\begin{array}{lllllllll}\mathrm{X} 1145.15 & 83 . & 539.6 & 1594.0 & 2350.0 & 1900.0 & 2160.0 & .00000 & .00\end{array}$

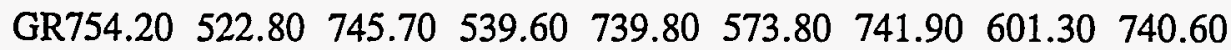
.630 .10

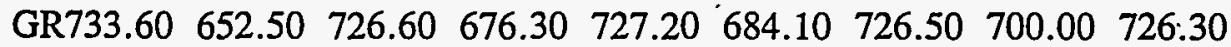

711.30

GR725.60 723.00 $724.90 \quad 738.00 \quad 724.70 \quad 755.50 \quad 724.40 \quad 763.40 \quad 723.50$ 778.00

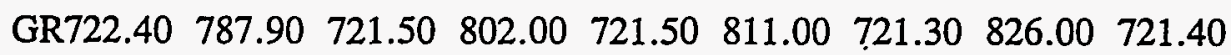
835.00

$\begin{array}{lllllll}\text { GR721.60 } 849.50 \quad 721.00 & 858.00 & 720.50 & 867.00 & 719.30 & 880.00 & 718.50\end{array}$ 890.00

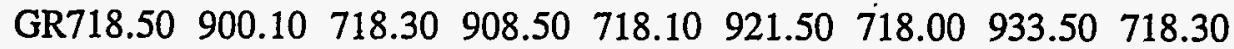

943.10

GR718.80 $956.00 \quad 718.80 \quad 963.80 \quad 719.10 \quad 976.00 \quad 718.80 \quad 985.30 \quad 718.50$

993.00

GR718.50 $1006.60718 .401016 .50718 .301028 .50718 .401041 .00 \quad 718.30$ 1049.80

GR718.70 $1063.50718 .801070 .50718 .701081 .00 \quad 718.801091 .10 \quad 718.80$ 1099.00

GR719.00 1112.30 $719.201123 .50 \quad 719.301134 .00 \quad 719.501145 .00 \quad 719.80$ 1157.00

GR720.10 $1169.00 \quad 720.401177 .80 \quad 720.801192 .50 \quad 721.101201 .50 \quad 721.40$ 1214.50

GR721.80 1224.10 $722.301237 .00 \quad 722.501247 .50 \quad 722.601261 .50 \quad 722.90$ 1271.30

$\begin{array}{lllllll}\text { GR723.30 } 1286.00 \quad 723.40 & 1296.00 & 723.80 & 1306.50 & 724.10 & 1320.40 \quad 724.70\end{array}$ 1331.00

GR724.90 $1346.60 \quad 724.901362 .00 \quad 725.50 \quad 1372.50 \quad 725.701386 .00 \quad 725.20$ 1398.50

GR724.40 $1411.00 \quad 723.601423 .20 \quad 723.401434 .00 \quad 725.001449 .00 \quad 727.50$ 1462.00

GR728.60 1477.30 $728.001494 .90 \quad 730.101508 .00 \quad 732.201519 .80 \quad 735.00$ 1542.40

GR737.70 1570.70748 .901594 .00755 .101619 .60 
$\begin{array}{lllllllll}\mathrm{X} 1145.22 & 83 . & 706.3 & 1872.6 & 360.0 & 350.0 & 360.0 & .00000 & .00\end{array}$

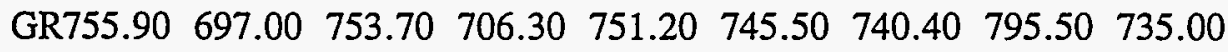
824.00

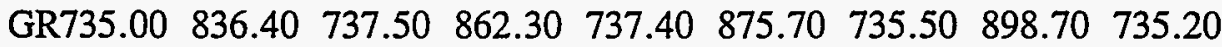

915.30

GR737.00 $942.80 \quad 739.30 \quad 954.00 \quad 740.70 \quad 980.50 \quad 740.70 \quad 1002.50 \quad 740.40$ 1037.80

GR735.10 $1050.30 \quad 728.901066 .10 \quad 726.701081 .00 \quad 726.601092 .30 \quad 725.40$ 1107.60

GR724.40 1118.00 723.40 1134.00722 .901146 .00722 .101159 .00721 .00 1176.00

GR720.90 $1183.30 \quad 720.201198 .00 \quad 719.801205 .40 \quad 719.101220 .50 \quad 718.60$ 1227.50

GR717.90 $1243.50 \quad 717.501250 .40 \quad 717.201260 .00 \quad 717.201271 .00 \quad 717.30$ 1278.50

GR716.80 $1291.50 \quad 716.501300 .50 \quad 717.201313 .00 \quad 718.001324 .50 \quad 718.00$ 1334.50

GR717.70 $1347.50717 .601357 .10 \quad 717.601370 .50 \quad 718.001378 .50 \quad 717.40$ 1389.00

GR717.10 1399.10 717.00 $1406.50 \quad 717.101421 .00 \quad 717.901429 .50 \quad 716.90$ 1442.00

GR716.40 1451.50 $716.901462 .60716 .901473 .00716 .701484 .00 \quad 716.30$ 1495.00

GR716.20 1505.50 716.30 1519.00 716.30 1526.30 716.90 1541.00 716.80 1547.80

GR716.50 $1559.00 \quad 716.901568 .30 \quad 717.201575 .50 \quad 718.301590 .00 \quad 718.60$ 1599.00

GR718.80 $1611.00719 .301623 .00719 .801634 .00 \quad 720.501645 .50 \quad 721.40$ 1656.90

GR722.20 $1671.00722 .701681 .50 \quad 723.301694 .00 \quad 724.801706 .50 \quad 727.70$ 1720.80

GR730.70 $1735.50731 .80 \quad 1747.70 \quad 733.00 \quad 1768.10 \quad 735.201792 .50 \quad 736.20$ 1804.90

GR737.80 1820.30755 .301872 .60760 .001890 .00

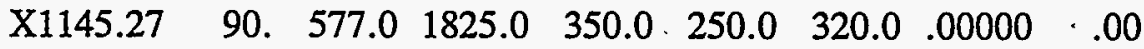

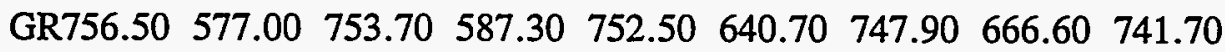
695.40

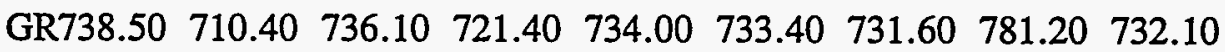
814.80

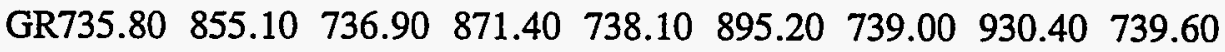
965.50

GR738.70 $975.10739 .70 \quad 983.50739 .401024 .20738 .601052 .10 \quad 736.90$ 1064.20

GR735.80 1074.40 734.10 1086.50 728.90 1103.00 727.80 1111.00 727.50 
1121.00

GR727.10 1132.00 726.70 1142.00 726.00 1152.00 725.40 1162.00 725.00 1172.00

GR724.90 1184.00 723.701193.00 723.10 1204.00 722.601214 .00721 .90 1224.00

GR721.30 $1235.00 \quad 720.501247 .00 \quad 719.401256 .00718 .801267 .00717 .90$ 1279.00

GR717.50 1288.00 717.10 $1298.00 \quad 717.601310 .00 \quad 717.301320 .00 \quad 717.90$ 1329.00

GR717.30 1340.00 717.70 1350.00 716.90 1360.00 716.10 1369.00 716.60 1380.00

GR716.90 $1390.00716 .401401 .00 \quad 715.901410 .00 \quad 715.101421 .00 \quad 715.50$ 1434.00

GR716.90 $1444.00 \quad 714.901454 .00 \quad 714.801464 .00 \quad 714.901474 .00 \quad 715.60$ 1484.00

GR715.70 $1493.00 \quad 715.401503 .00 \quad 715.201515 .00 \quad 715.801528 .00 \quad 715.80$ 1537.00

GR716.60 1549.00 716.50 1559.00 716.60 1572.00 $716.501584 .00 \quad 716.50$ 1595.00

GR717.10 $1606.00718 .201617 .00 \quad 719.001627 .00 \quad 719.401637 .00 \quad 720.00$ 1647.00

GR720.60 1657.00 721.00 $1664.00 \quad 722.401669 .00 \quad 723.701675 .00 \quad 725.40$ 1680.00

GR726.60 $1683.00727 .501686 .00 \quad 729.301694 .00 \quad 730.901701 .00733 .00$, 1712.80

GR735.70 $1727.90 \quad 736.601740 .90 \quad 740.50 \quad 1771.80 \quad 744.50 \quad 1791.10 \quad 755.00$ 1825.00

$\begin{array}{lllllllll}X 1145.31 & 87 . & 801.6 & 1902.0 & 350.0 & 220.0 & 270.0 & .00000 & .00\end{array}$

$\begin{array}{lllllll}\text { GR756.00 } 749.00 \quad 753.10 & 763.40 & 750.10 & 777.80 & 746.30 & 793.40 & 746.10\end{array}$

801.60

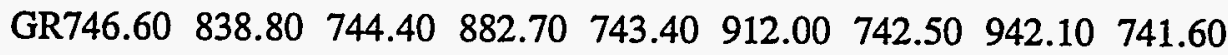

988.30

GR741.60 993.70741 .501031 .80741 .801045 .60741 .401075 .70739 .50

1095.70

GR738.10 $1127.20740 .001139 .30736 .201159 .10 \quad 735.801175 .00734 .70$ 1195.70

GR733.60 1208.60 732.40 1221.20 730.10 1238.30 729.50 1253.80 729.80 1265.00

GR729.30 $1276.60728 .901290 .70 \quad 729.101303 .90 \quad 729.001316 .10 \quad 729.20$ 1327.40

GR729.10 $1341.30 \quad 727.701357 .10 \quad 727.70 \quad 1365.10 \quad 727.201378 .90 \quad 726.80$ 1389.40

GR726.60 $1400.30 \quad 725.801411 .80725 .001422 .60 \quad 723.901433 .40 \quad 724.30$ 1442.90

GR723.90 $1455.50 \quad 723.201463 .10 \quad 722.30 \quad 1476.30 \quad 722.00 \quad 1484.40 \quad 721.40$ 1495.40 
GR720.60 1506.80 $719.901515 .40 \quad 718.901523 .60717 .601533 .50716 .90$ 1544.40

GR717.10 $1550.00 \quad 716.301558 .80 \quad 715.301566 .80 \quad 715.501578 .10 \quad 714.70$ 1584.00

GR715.20 $1594.90 \quad 715.101599 .40 \quad 714.901606 .80715 .001615 .70714 .80$ 1624.10

GR714.80 1632.60 $715.401644 .90 \quad 715.901648 .90 \cdot 715.101656 .30 \quad 715.70$ 1664.50

GR715.80 1674.60 $715.201681 .30 \quad 715.101690 .00 \quad 715.50 \quad 1697.50 \quad 716.60$ 1703.40

GR717.70 $1714.50 \quad 718.101719 .70 \quad 718.90 \quad 1732.40 \quad 719.30 \quad 1742.00 \quad 719.80$ $1751: 10$

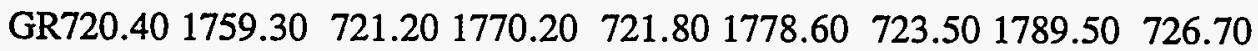
1803.20

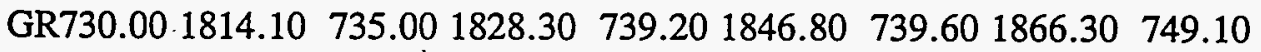
1902.00

GR751.80 1908.70755 .001925 .00

$\begin{array}{lllllllll}\mathrm{X} 1145.38 & 83 . & 605.6 & 2074.3 & 300.0 & 240.0 & 280.0 & .00000 & .00\end{array}$

GR756.10 $605.60 \quad 746.00 \quad 643.20 \quad 743.40 \quad 682.30 \quad 743.90 \quad 726.70 \quad 741.60$

743.10

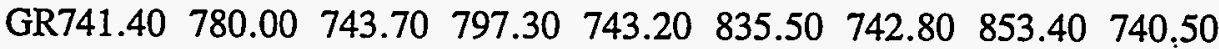

882.40

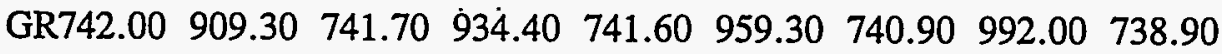

1012.10

GR739.70 $1036.30738 .701060 .90 \quad 740.401079 .90 \quad 738.801107 .70 \quad 738.60$

1146.60

GR738.60 $1155.80 \quad 738.501183 .40 \quad 738.301200 .30 \quad 738.101217 .20 \quad 737.50$

1243.30

GR737.10 $1258.40736 .501286 .30 \quad 736.40 \quad 1291.50 \quad 734.401326 .90 \quad 733.80$ 1338.70

GR732.30 1364.70 $730.901390 .30 \quad 729.401401 .00 \quad 728.601416 .60 \quad 728.70$ 1433.00

GR728.60 $1450.80729 .101465 .30 \quad 728.701483 .30 \quad 729.401498 .00 \quad 728.80$ 1514.30

GR727.80 $1529.00 \quad 725.001546 .30 \quad 723.70 \quad 1559.30 \quad 721.80 \quad 1572.50 \quad 719.80$ 1585.00

GR717.70 $1600.00716 .401609 .50 \quad 715.301621 .30 \quad 713.501631 .80 \quad 712.80$ 1642.00

GR712.50 1654.00 712.20 1665.50 712.50 $1674.00 \quad 712.501687 .70 \quad 712.30$ 1696.60

GR711.90 $1710.50712 .101716 .80712 .401727 .00 \quad 713.901739 .50 \quad 714.00$ 1750.30

GR714.20 $1761.30 \quad 715.00 \quad 1772.50 \quad 715.501783 .80 \quad 715.90 \quad 1796.30 \quad 717.10$ 1807.00

GR718.90 $1819.50719 .801830 .00 \quad 720.501845 .30 \quad 721.001857 .00 \quad 721.50$ 
1870.50

GR721.90 $1881.50722 .401896 .30 \quad 723.301909 .00 \quad 725.501927 .80 \quad 727.80$ 1938.00

GR730.30 1950.50 735.50 1973.30 $739.401994 .40 \quad 740.602022 .10 \quad 742.30$ 2057.10

GR750.90 2074.30 750.902077.60 760.00 2095.00

$\begin{array}{lllllllll}\mathrm{X} 1145.61 & 85 . & 548.1 & 1616.2 & 1450.0 & 1200.0 & 1320.0 & : 00000 & .00\end{array}$

GR756.80 $500.00753 .20 \quad 521.10 \quad 751.90 \quad 529.00 \quad 748.90 \quad 548.10 \quad 741.60$ 587.80

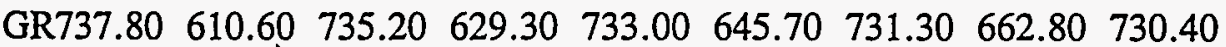
673.50

GR729.40 $688.60 \quad 728.90 \quad 700.70 \quad 728.60 \quad 711.30 \quad 728.60 \quad 726.00 \quad 728.70$ 737.20

GR729.10 751.60 $729.70 \quad 766.00 \quad 730.00 \quad 778.70 \quad 729.60 \quad 790.90 \quad 729.60$ 806.60

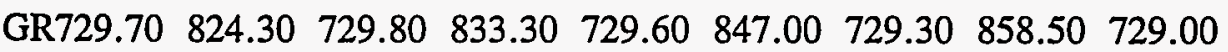
869.20

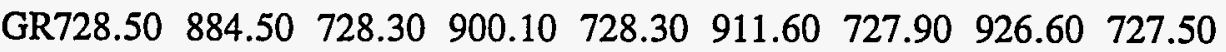
937.00

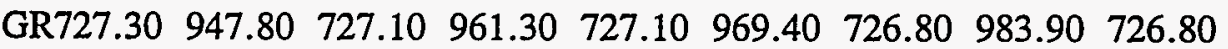
995.10

GR726.60 $1007.90 \quad 726.70 \quad 1024.50 \quad 726.50 \quad 1033.10 \quad 726.50 \quad 1048.60 \quad 726.50$ 1056.10

GR726.30 $1073.20726 .301080 .60726 .501096 .30 \quad 726.301103 .00 \quad 726.30$ 1116.40

GR726.20 $1126.30 \quad 726.201137 .60 \quad 726.201150 .80 \quad 726.10 .1166 .10 \quad 726.00$ 1174.20

GR725.80 1190.20 $725.701196 .40 \quad 725.901210 .40 \quad 726.101219 .90 \quad 725.70$ 1232.40

GR725.30 1243.80 725.00 1257.10 $724.901265 .20 \quad 724.50 \quad 1276.30 \quad 724.40$ 1287.50

GR724.20 $1298.80 \quad 723.701309 .60 \quad 723.001325 .40 \quad 722.801331 .50 \quad 723.10$ 1342.60

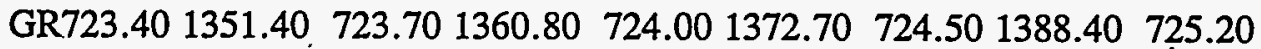
1396.40

GR725.80 1412.90 $726.301419 .20 \quad 727.201436 .60 \quad 727.601443 .10 \quad 728.20$ 1457.10

GR729.50 $1467.00 \quad 730.40 \quad 1480.60 \quad 730.301495 .60 \quad 732.70 \quad 1516.40 \quad 734.40$ 1525.10

GR736.70 $1537.40 \quad 739.201564 .10 \quad 740.201578 .60 \quad 744.201616 .20 \quad 760.00$ 1660.00

$\begin{array}{lllllllll}\mathrm{X} 1146.33 & 76 . & 535.6 & 1459.0 & 3400.0 & 3500.0 & 3460.0 & .00000 & .00\end{array}$

GR750.80 $535.60 \quad 747.30 \quad 569.60 \quad 746.90 \quad 605.70 \quad 738.10 \quad 635.40 \quad 736.80$ 650.40 


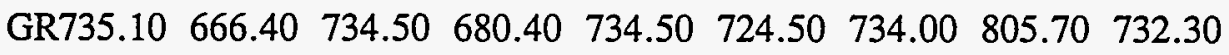
834.20

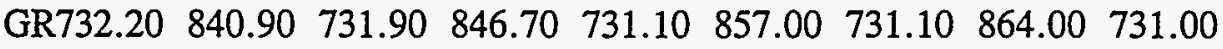
871.00

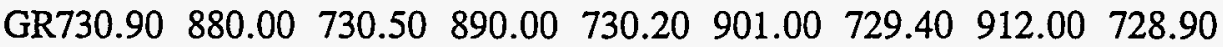
923.00

$\begin{array}{lllllll}\text { GR728.30 } 933.00 \quad 728.20 & 943.00 & 727.70 & 951.00 & 726.90 & 961.00 & 726.70\end{array}$ 971.00

GR726.50 980.00 726.20 990.00 726.10 $1001.00 \quad 725.801011 .00 \quad 725.30$ 1021.00

GR725.20 $1030.00725 .001039 .00724 .601048 .00 \quad 724.301057 .00 \quad 723.80$ 1067.00

GR723.50 $1078.00722 .901088 .00722 .101097 .00721 .401106 .00 \quad 721.10$ 1116.00

GR720.40 1124.00 $719.401134 .00718 .301143 .00 \quad 717.601153 .00 \quad 716.80$ 1163.00

GR716.00 $1173.00715 .301182 .00714 .501192 .00 \quad 714.001202 .00 \quad 714.00$ 1213.00

GR713.80 1224.00 714.10 $1234.00 \quad 720.401254 .00 \quad 719.601265 .00 \quad 720.50$ 1275.00

GR721.10 $1287.00721 .701299 .00723 .801311 .00 \quad 723.101323 .00 \quad 724.90$ 1334.00

GR725.90 $1345.00726 .301354 .00726 .401363 .00 \quad 727.001368 .00 \quad 727.80$ 1375.00

GR729.30 1416.80 $734.401441 .30 \quad 737.001449 .50 \quad 742.80 \quad 1459.00 \quad 743.30$ 1469.10

GR744.40 1477.50 748.20 $1492.60 \cdot 752.301509 .40 \quad 754.101516 .40 \quad 756.90$ 1527.80

GR760.00 1540.00

$\begin{array}{llllllllll}X 1146.87 & 70 . & 525.6 & 1273.2 & 2700.0 & 3200.0 & 3000.0 & .00000 & .00\end{array}$

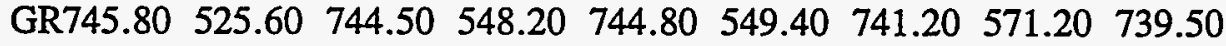
580.50

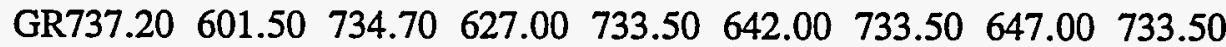
653.00

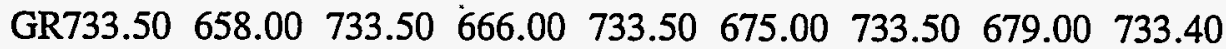
691.00

GR733.00 $699.00 \quad 732.20 \quad 708.00 \quad 731.10 \quad 718.00 \quad 730.20 \quad 730.00 \quad 729.10$ 739.00

GR728.70 $749.00 \quad 728.40 \quad 762.00 \quad 728.10 \quad 774.00 \quad 727.50 \quad 784.00 \quad 727.30$ 792.00

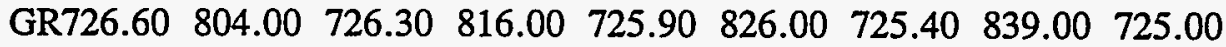
849.00

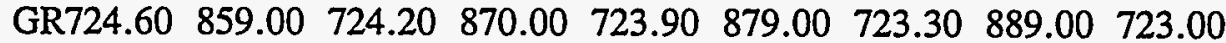
900.00

$\begin{array}{lllllll}\text { GR722.40 } 910.00 \quad 722.20 & 920.00 & 721.80 & 929.00 & 720.80 & 941.00 & 720.40\end{array}$ 
953.00

$\begin{array}{lllllll}\text { GR720.00 } 963.00 \quad 719.60 \quad 974.00 & 720.90 \quad 984.00 \quad 719.00 & 996.00 \quad 719.30\end{array}$ 1007.00

GR720.90 $1020.00722 .101030 .00721 .701040 .00 \quad 721.701051 .00 \quad 722.10$ 1064.00

GR722.50 $1077.00 \quad 722.501088 .00 \quad 723.501100 .00 \quad 723.801116 .00 \quad 722.70$ 1127.00

GR723.40 $1138.00724 .601148 .00 \quad 724.401157 .00 \quad 724.701165 .00 \quad 724.70$ 1171.00

GR725.50 1175.00 725.50 1179.00 731.80 1203.00 737.20 1227.50 738.30 1236.30

GR741.80 $1244.60 \quad 743.00 \quad 1254.60 \quad 745.801267 .60 \quad 748.00 \quad 1273.10 \quad 748.90$ 1273.20

$\begin{array}{lllllllll}\mathrm{X} 1147.47 & 28 . & 500.0 & 1580.0 & 3100.0 & 3450.0 & 3280.0 & .00000 & .00\end{array}$

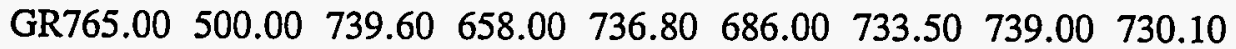

800.00

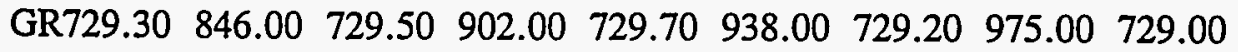

1021.00

GR728.80 $1062.00728 .901096 .00 \quad 728.801135 .00 \quad 728.301176 .00 \quad 728.20$ 1215.00

GR727.80 1265.00 $729.401317 .00 \quad 734.601363 .00 \quad 737.401409 .00 \quad 737.80$

1444.00

GR739.60 $1452.00 \quad 744.501482 .00 \quad 745.101484 .00 \quad 748.00 \quad 1516.00 \quad 750.50$.

1518.00

GR753.40 1541.00 759.001557.00765.00 1580.00 :

$\begin{array}{lllllllll}X 1148.09 & 20 . & 500.0 & 1520.0 & 3000.0 & 3050.0 & 3040.0 & .00000 & .00\end{array}$

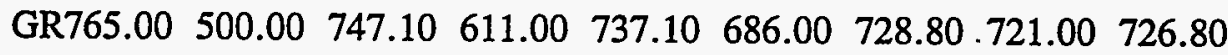

773.00

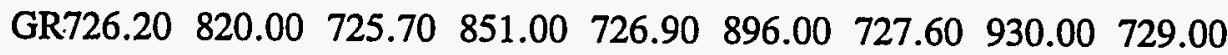

973.00

GR730.10 1019.00730 .401051 .00730 .701092 .00731 .601152 .00733 .30

1207.00

GR735.10 $1262.00736 .301307 .00 \quad 739.301356 .00 \quad 741.101394 .00 \quad 765.00$ 1520.00

$\begin{array}{lllllllll}X 1148.83 & 26 . & 500.0 & 1545.0 & 3900.0 & 3300.0 & 3700.0 & .00000 & .00\end{array}$

GR767.40 $500.00 \quad 749.60 \quad 595.00 \quad 740.60 \quad 639.00 \quad 739.30 \quad 655.00 \quad 739.90$ 696.00 .

GR739.80 $788.00 \quad 739.40 \quad 828.00 \quad 738.50 \quad 863.00 \quad 737.00 \quad 900.00 \quad 735.40$ 930.00

GR733.50 958.00 $732.60988 .00732 .00 \quad 1008.00 \quad 731.90 \quad 1030.00 \quad 731.90$ 1056.00

GR732.00 1088.00 732.00 $1148.00732 .601198 .00 \quad 733.401232 .00 \quad 734.00$ 1279.00 
GR735:20 $1333.00 \quad 737.40 \quad 1385.00 \quad 741.601446 .00 \quad 743.40 \quad 1456.00 \quad 752.80$

1499.00

GR770.00 1545.00 


\section{Appendix D}

Subregions of the Lower Granite Reservoir 


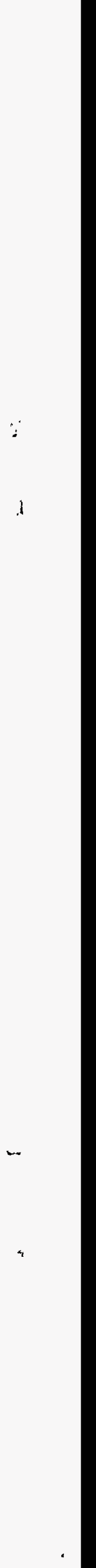




\section{Appendix D}

\section{Subregions of the Lower Granite Reservoir}

Coordinates of the eight sub-regions of the Lower Granite Reservoir . Subsetting done as a result of software limitations of PV-WAVE. Sub-regions were merged together using GRASS.

\begin{tabular}{ll} 
FILE NUMBER & NORTHSOUTHEASTWEST \\
\hline 1 & 50100049200027850002770000 \\
2 & 49302047700027930202780000 \\
3 & 47810046670028059002786700 \\
4 & 47192045590028199102804700 \\
5 & 45690043110028252002815600 \\
6 & 43211041720028318002819800 \\
7 & 41970041160028630002840800 \\
8 & 42082040540028767002862000 \\
9 & 41830040924028419602825460
\end{tabular}





\section{Appendix E}

Conversion of GRASS Raster Files to PV-WAVE Input 


\section{Appendix E}

\section{Conversion of GRASS Raster Files to PV-WAVE Input}

grassras_to_PV-WAVE.f

generate a PV-WAVE input file with latitude and longitude coordinates from a GRASS raster file.

C input the raster files NW Corner Coordinates and the pixel

C resolution

C

C If the input value is equal to the 'ZOT' value, it is assumed to

C be masked into the value of ZERO.

C

C

implicit none

$\begin{array}{ll}\text { integer } & \text { maxlin } \\ \text { parameter } & (\operatorname{maxlin}=4096)\end{array}$

C

byte buffb(maxlin), bval

integer*2 buffi(maxlin), Zshort(2), bint

integer*4 buffi4(maxlin)

character $* 40$ fnin, fnout

double precision North, west, X,Y,Z, zot

real pixres

C

integer form, colms, lines, inrec, iln, ic, ipts_out, i, Ztem

C

equivalence (Ztem,Zshort(1))

C

$\mathrm{zot}=666.0$

write $(*, *)$ ',

write $(*, *)$ ' Enter GRASS Raster file name'

$\operatorname{read}(*, 100)$ fnin

write $\left(*,{ }^{*}\right)$ ' Enter number of columns and lines'

$\operatorname{read}(*, *)$ colms, lines

78 write $\left(*,{ }^{*}\right)$ ' Choose Format: 1) byte, 2) Integer*2, 4) I*4'

$\operatorname{read}(*, *)$ form

C

if(form.lt. 1 .or. form.gt. 4 .or. form.eq.3) go to 78 
write $\left(*,{ }^{*}\right)^{\prime}$ Enter NW Corner Coordinates (west, north)'

$\operatorname{read}(*, *)$ west, north

write $\left(^{*}, *\right)$ ' Enter Pixel Resolution (Coordinate Units)'

C

$\operatorname{read}(*, *)$ pixres

write $(*, *)^{\prime}$,

write $(*, *)^{\prime}$ Enter Output file name'

C.

read $(*, 100)$ fnout

inrec $=$ colms $*$ form

open $(11$, file $=$ fnin, status $=$ 'old', access $=$ 'direct',

+ form $=$ 'unformatted', $r e c l=$ inrec)

C

open $(21$, file $=$ fnout,status =',unknown')

ipts_out $=0$

$\mathrm{Y}=$ north

C

do 10 iln $=1$,lines

$\mathrm{X}=$ west

if(form.eq. 1) then

call read_byte_dbin(buffb,colms, 11,iln)

else if(form.eq. $\overline{2}$ ) then

call read_i2_dbin(buffi,colms, 11,iln)

else if(form.eq.4) then

call read i4 dbin(buffi 4,colms, 11,iln)

endif

C

do 12 ic $=1$, colms

if(form.eq. 1) then

if(buffb(ic).ne.0) then

$$
\begin{aligned}
& \text { bval }=\text { buffb(ic) } \\
& \text { bint }=\text { bval }+256 \\
& Z=\text { float(bint) } \\
& \text { if(Z.eq.zot) } Z=0.0 \\
& \text { write( } 21, *) X, Y, Z \\
& \text { ipts_out }=\text { ipts_out }+1 \\
& \text { if(mod(ipts_out,200).eq.0) }
\end{aligned}
$$

$+\quad$ write $(*, *)^{\prime}$ At output point ',ipts_out,' $\mathrm{X}, \mathrm{Y}, \mathrm{Z}=$, $, \mathrm{X}, \mathrm{Y,Z}$

endif

else if(form.eq.2) then

if(buffi(ic).ne.0) then

$$
\begin{aligned}
& Z=\text { float(buffi(ic)) } \\
& \text { if( } Z \text {.eq.zot) } Z=0.0 \\
& \text { write( } \left.21,{ }^{*}\right) X, Y, Z \\
& \text { ipts_out = ipts_out }+1 \\
& \text { if(mod(ipts_out,200).eq.0) }
\end{aligned}
$$

$+\quad$ write $\left(*,{ }^{*}\right)$ ' At output point ',ipts_out,' $\mathrm{X}, \mathrm{Y}, \mathrm{Z}={ }^{\prime}, \mathrm{X}, \mathrm{Y}, \mathrm{Z}$ 


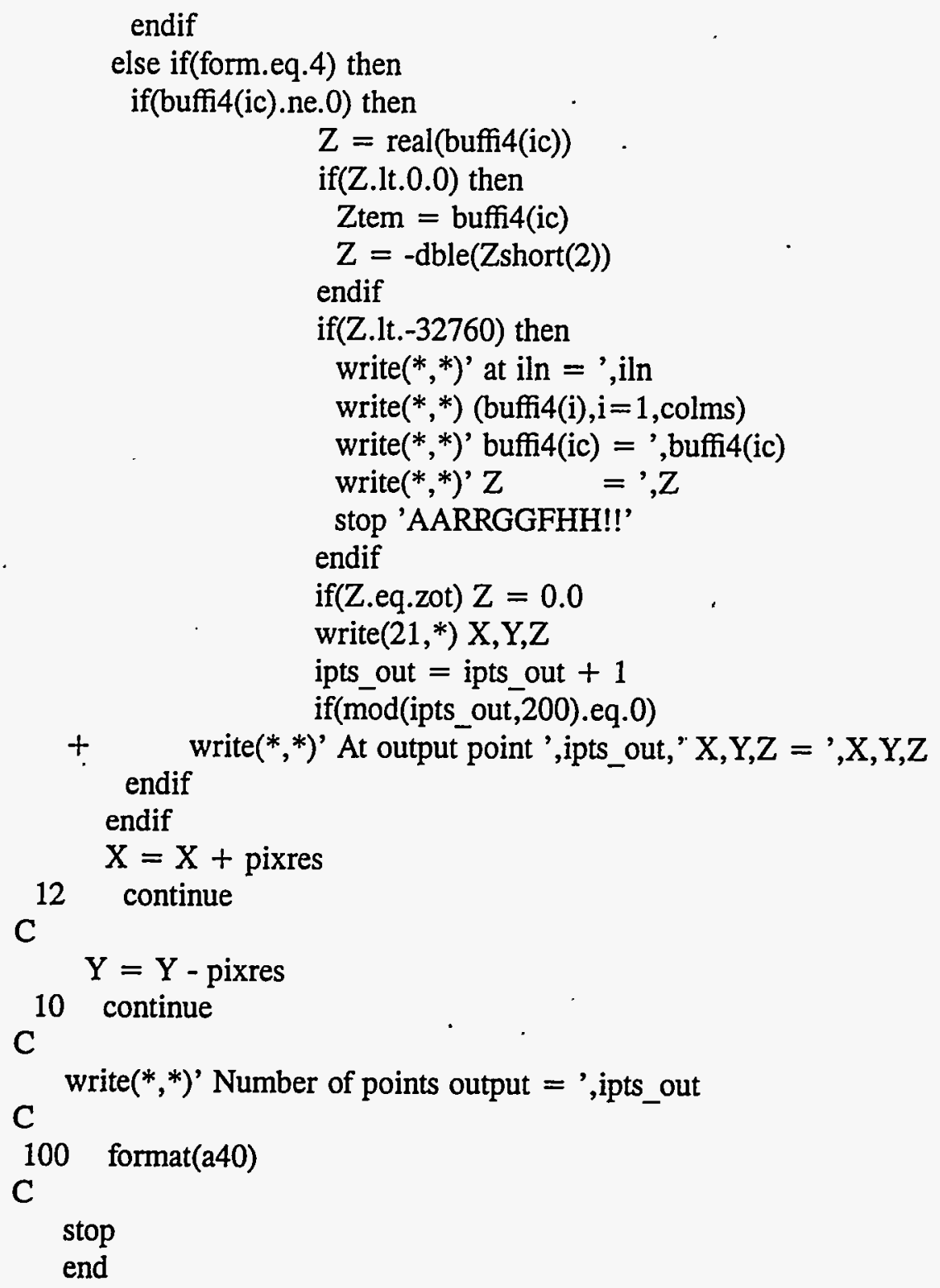



Appendix F

Interpolation of Depth Values for Channel Centerline 



\section{Appendix F}

\section{Interpolation of Depth Values for Channel Centerline}

This appendix contains the source code and an example log from tgridgen_xypts, the program used to interpolate depth values along the river channel line. Interpolates a regular grid from irregular latitude/ longitude coordinates and their depths.

I. Source

C program: tgridgen_xypts

C function: interpolation of general surface from irregularly

C spaced point

C MODIFIED by LGM Mar 5, 1993 to interpret XY input points

C from the input data file of $\mathrm{XYZ}$ data

$\mathrm{C}$ implicit none integer npoints, $\mathrm{nx}, \mathrm{ny}, \mathrm{nz}$

C parameter (npoints $=10000, n x=200, n y=200, n z=200$ )

real $\quad \operatorname{ttab}(3$, npoints)

integer*4 indary(nx,ny,nz), head(50)

integer*4 ntmx, ip, nstr, ntot, maxpoints, ind

character*40 nam1, namout, namxy

logical endfile

real zthresh

C

integer inxmx,inymx,inmx, rdmx, ncolq,nlinq, ic,

$+\quad$ icol,ilin, ncol,nlin, nsmp0, ift, nn, ipr,

$+\quad \mathrm{i}, \mathrm{j}, \mathrm{k}, \mathrm{ix}, \mathrm{iy}$, nncol,nnlin, nsum, ixi,

$+\quad$ iyi, iib,iie, ijb,ije, npt, ix_mn,ix_mx,iy_mn,

$+\quad$ iy_mx, max_srad, iun, oun, count

real sizeq,dxq,dyq, xdimq,ydimq,bpln,tmax,

$+\quad \mathrm{x} 00 \mathrm{q}, \mathrm{y} 00 \mathrm{q}, \mathrm{years}, \mathrm{zbias}$, gispc, xidm,yidm, $\mathrm{x} 0 \mathrm{i}$,

$+\quad y 0 i, x o d m, y 0 d m, x 00, y 00$, radin,pwr,

$+\quad x \min , x \max , y \min , y \max , z \min , z \max , \mathrm{x}, \mathrm{y}, \mathrm{z}, \mathrm{tmax}$,

$+\quad$ rdsch, wtsum, tsum, xt,yt,tp,dxt,dyt,rt, wt, twt, Z,

$+\quad$ input_zmax, input_zmin

C

logical endfile, found

common /bk/ ncolq,nlinq,sizeq,dxq,dyq,xdimq,ydimq,bpln,tmax

$1 \quad$,x00q,y00q,years 
C

C

equivalence (head,ncolq)

ntmx $=$ npoints
inxmx $=n x$
inymx $=n y$
inmx $=n z-1$
rdmx $=1200$

write $(*, *)$, ,

write $(*, *)^{\prime}$ max number of input points $=$ ',ntmx

write $(*, *)^{\prime}$ ' max size of index array $=$ ', inxmx, inymx, inmx

C

write $(*, *)$ ' $\max$ initial search radius $=$ ', $r \mathrm{dmx}$

1 write $(*, *)$, '

write $(*, *)^{\prime}(1)$ - specify input $\mathrm{x}, \mathrm{y}, \mathrm{z}$ file'

write $(*, *) '(2)$ - specify index array'

write $\left(*,{ }^{*}\right) '(3)$ - specify input XY Coordinate file and Output file'

write $(*, *)^{\prime}(4)$ - set interpolation parameters'

write $(*, *) '(5)$ - read XYZ point data file'

write $(*, *)^{\prime}(6)$ - generate output XYZ file'

write $(*, *)^{\prime}(7)$ - stop'

$\operatorname{read}(*, *)$ ic

if(ic.eq.1) then .

write $(*, *)$ 'enter $\mathrm{x}-\mathrm{y}-\mathrm{z}$ data input file name'

$\operatorname{read}(*, 100)$ nam1

open(unit $=1$, name $=$ nam 1 ,type $=$ 'old',form ='formatted')

write $(*, *)$ ' enter $\mathrm{z}$ bias value,(Add to Input $\mathrm{Z}$ Values)'

$\operatorname{read}(*, *)$ zbias

write $(*, *)$ ' Enter Minimum and Maximum $\mathrm{Z}$ value to be used in Interp'

Cic

$\operatorname{read}(*, *)$ input_zmin, input_zmax

else if(ic.eq.2) then

write $(*, *)$ 'enter index grid spacing '

$\operatorname{read}(*, *) \operatorname{gispc}$

write $(*, *)$ 'enter x-dim , y-dim for index grid '

read $(*, *)$ xidm,yidm

icol $=$ int $(x i d m /$ gispc +.5$)$

ilin $=$ int (yidm/gispc +.5 )

icol $=\min ($ icol,inxmx)

ilin $=\min ($ ilin, inymx $)$

write $(*, *)$ 'icol =',icol,' ilin =', ilin

write $(*, *)$ ' Enter Minimum X Coordinate'

$\operatorname{read}(*, *) \times 0 \mathrm{i}$

write $(*, *)$ ' Enter Maximum Y coordinate'

$\operatorname{read}(*, *)$ y0i

C $\quad \mathrm{x} 0 \mathrm{i}=0$ 
C $\mathrm{yO} \mathrm{i}=$ yidm

Cic

else if(ic.eq.3) then

write $(*, *)$ '

write $(*, *)^{\prime}$ 'Enter XY Input file of points to interpolate'

$\operatorname{read}(*, 100)$ namxy

iun $=20$

open(iun, file $=$ namxy,status ='unknown')

write $(*, *)$ ' Enter Output File Name'

$\operatorname{read}(*, 100)$ namout

oun $=31$

Cic

open(oun, file = namout,status ='unknown')

else if(ic.eq.4) then

write $(*, *)$ 'enter initial search radius (XY Input Units)'

$\operatorname{read}(*, *)$ radin

write $(*, *)^{\prime}$ Enter MAX search radius factor'

write $(*, *)^{*}$ (multiply by initial search radius)'

$\operatorname{read}(*, *)$ max_srad

write $(*, *)$ 'enter min sample count'

$\operatorname{read}(*, *)$ nsmp0

write $\left({ }^{*},{ }^{*}\right)$ 'enter pwr in weight function: $1 / \mathrm{r}^{* *}$ pwr'

$\operatorname{read}(*, *)$ pwr

write $(*, *)^{*}$ enter minumum $\mathrm{z}$ threshold value'

read $(*, *)$ zthresh

Cic

else if(ic.eq.5) then

endfile $=$.false.

ip $=0$

$x \min =1.0 \mathrm{e}+24$

$x \max =-x \min$

$y \min =x \min$

$y \max =x \max$

$\operatorname{zmin}=x \min$

$\mathrm{zmax}=\mathrm{xmax}$

write $(*, *)$ ' enter max number points to read $(-1=\text { all })^{\prime}$

$\operatorname{read}(*, *)$ maxpoints

if(maxpoints.eq. -1$)$ maxpoints $=$ npoints

write $(*, *)$ ' '

do 450 while(.not. endfile)

if(ip.ge.maxpoints) go to 99

$\operatorname{read}(1, *$,end $=99) \mathrm{x}, \mathrm{y}, \mathrm{z}$

if(z.le.input_zmax .and. z.ge.input_zmin) then

ip $=$ ip +1

if(mod(ip,500).eq.0) write(*,700) ip

$\operatorname{ttab}(1, \mathrm{ip})=\mathrm{x}$

$\operatorname{ttab}(2, \mathrm{ip})=y$ 


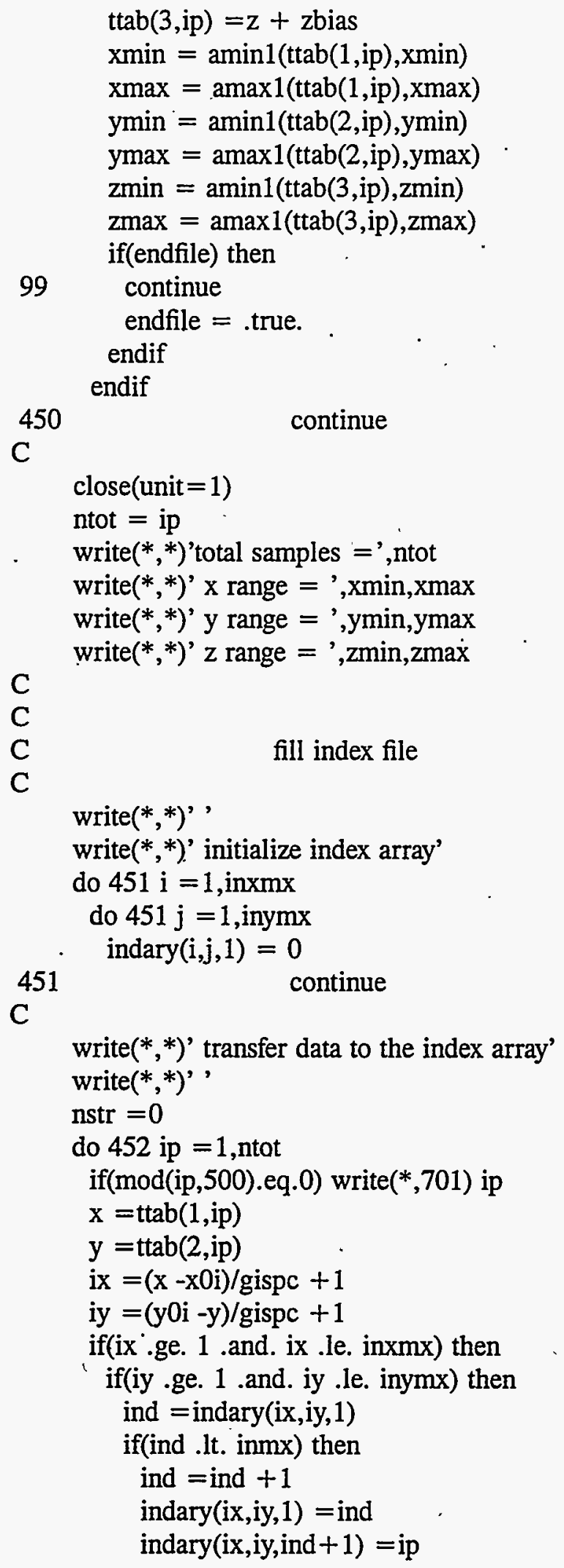

write $(*, *)$ ' transfer data to the index array' write $(*, *)$ ', 


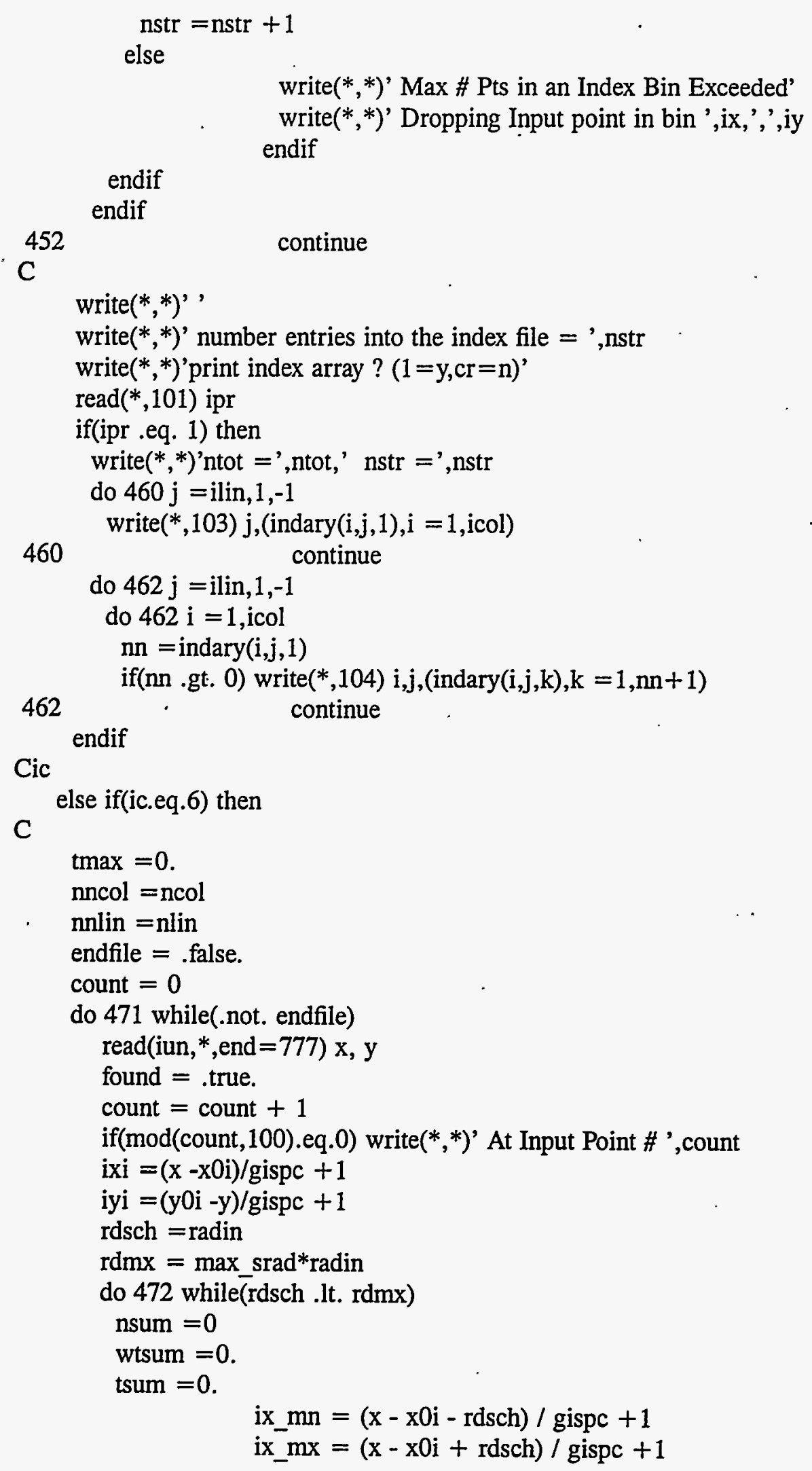




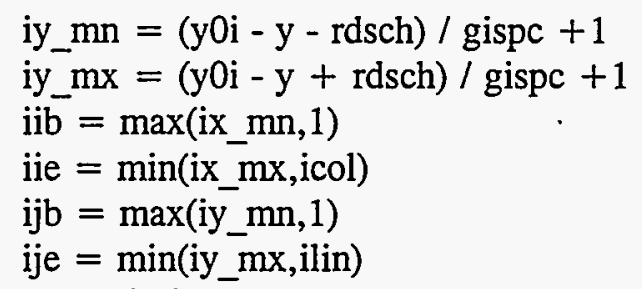

C write $(*, *)^{\prime}$ ind, $\mathrm{xt}, \mathrm{yt}, \mathrm{tp}, \mathrm{dxt}, \mathrm{dyt}=$ ', ind, $\mathrm{xt}, \mathrm{yt}, \mathrm{tp}, \mathrm{dxt}$, dyt

C write $(*, *)$ ' rt, wt,nsum $=$ ', rt, wt,nsum

C write $(*, *)^{\prime}$ ' wtsum,tsum $=$ ', wtsum,tsum

endif

474

continue

C

if(nsum .lt. nsmp0) then

$$
\text { rdsch }=1.5 * \text { rdsch }
$$

else

endif

$$
\mathrm{rdsch}=\mathrm{rdmx}
$$

472

continue

C 
if(nsum .ge. nsmp0) then

twt $=$ tsum/wtsum

else

$$
\text { twt }=\text { zthresh }
$$

write $(*, *)$ ' Minumum number of points not found for a point'

found $=$.false.

endif

$z=t w t$

tmax $=\operatorname{amax} 1(\operatorname{tmax}, \mathrm{twt})$

C write $(*, *)^{\prime}$ twt, tmax $=$ ', twt, tmax

C

C write $(*, *)^{\prime} \mathrm{X}, \mathrm{Y}, \mathrm{Z}={ }^{\prime}, \mathrm{x}, \mathrm{y}, \mathrm{z}$

C pause' hit $C$ to Continue' if(found) write(oun,888) $\mathrm{x}, \mathrm{y}, \mathrm{Z}$

if(endfile) then

777

endif

endfile $=$.true.

.471

continue

C

write(*,*)' ',count,' Input Points Interpolated'

close(oun)

close(iun)

Cc

C write-file-discriptor

C ************\#\#\#\#\#\#\#\#\#\#\#\#\#\#\#\#\#\$\$\$\$\$\$\$\$\$\$\$\$\$\$\$\$\$\&\&\&\&\&\&\&\&\&\&\&\&\&

C

write $(*, 107)$

if(ift.eq.1) then

write $(*, *)$ 'file type: formatted'

write $(*, 117)$ ncol,nlin

else if(ift.eq.2) then

write $(*, *) ' f i l e$ type: binary'

write $(*, 117)$ ncol,nlin

endif

C

write $(*, 109)$ x00,y00,xodm,yodm

C

write $(*, 111)$

write $(*, 112)$ nam1

C

write $(*, 113)$

C

write $(*, 114)$ radin,nsmp0,pwr

write $(*, 115)$

C

write $(*, 116)$ gispc,xidm,yidm, icol,ilin,x0i,y0i

C ************\#\#\#\#\#\#\#\#\#\#\#\#\#\#\#\#\$\$\$\$\$\$\$\$\$\$\$\$\$\$\$\$\$\&\&\&\&\&\&\&\&\&\&\&\&\& 


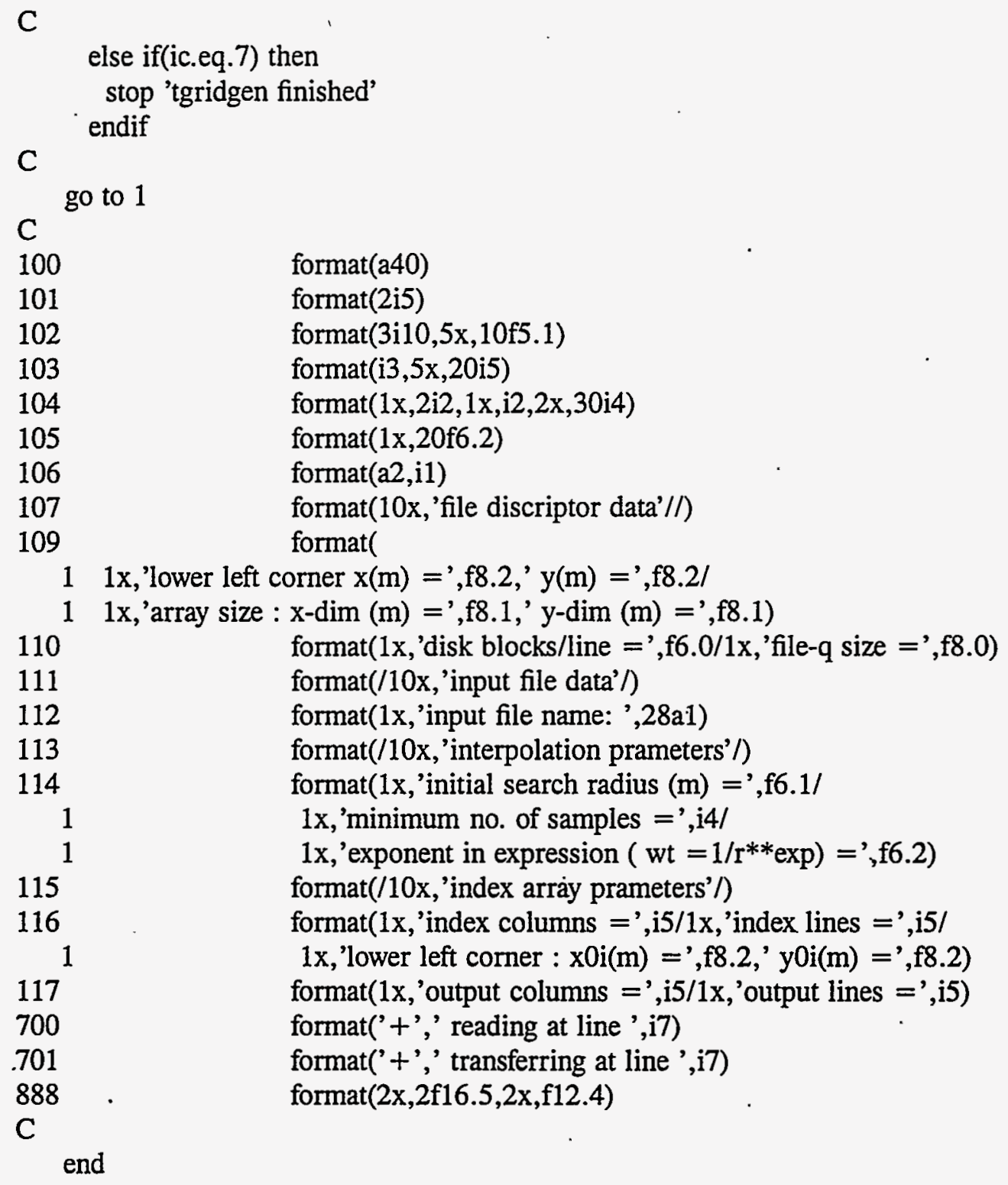

C

end format ( $1 \mathrm{x}$,'disk blocks/line $={ }^{\prime}, \mathrm{f6} 6.0 / 1 \mathrm{x}$,'file-q size $=$ ',f8.0) format(/10x,'input file data'/) format(1x,'input file name: ',28a1) format(/10x,'interpolation prameters'/) format(1x,'initial search radius $(\mathrm{m})=$, $\mathrm{f6} 6.1 /$ $1 \mathrm{x}$,'minimum no. of samples $=$ ',i4/ $1 \mathrm{x}$,'exponent in expression ( $\left.\mathrm{wt}=1 / \mathrm{r}^{* *} \exp \right)={ }^{\prime}, \mathrm{f6} 6.2$ ) format (/10x,'index array prameters'/) format $(1 \mathrm{x}$, 'index columns =',i5/1x,'index lines $=$ ',i5/ 1x,'lower left corner : $\mathrm{x} 0 \mathrm{i}(\mathrm{m})={ }^{\prime}, \mathrm{f} 8.2,{ }^{\prime}$ y $\left.0 \mathrm{i}(\mathrm{m})={ }^{\prime}, \mathrm{f} 8.2\right)$ format( $1 x$,'output columns $=$ ',i5/1x,'output lines =',i5) format(' + ',' reading at line ',i7) format(' + ',' transferring at line ',i7) format $(2 \mathrm{x}, 2 \mathrm{f} 16.5,2 \mathrm{x}, \mathrm{f} 12.4)$

\section{Example Log}

enter $x-y-z$ data input file name bath_and_xsect.data enter $\mathrm{z}$ bias value,(Add to Input $\mathrm{Z}$ Values)

0

Enter Maximum $\mathrm{Z}$ value to be used in Interp 500,638

index grid spacing

600 
enter $\mathrm{x}$-dim , $\mathrm{y}$-dim for index grid 105600,95100

Enter Minimum X Coordinate

2770500

Enter Maximum Y coordinate

500500

3

Enter XY Input file of points to interpolate channel 90.asc

Enter Output File Name

channel_90.xyzinterp.BS638

4

enter initial search radius (XY Input Units)

600

Enter MAX search radius factor

(multiply by initial search radius)

20

enter min sample count

5

enter pwr in weight function: $1 / \mathrm{r}^{* *} \mathrm{pwr}$

2

enter minumum $\mathrm{z}$ threshold value

0

$* * * * * * * * * * * * * * * * * * * * * * * * * * * * * * * * * * * * * * * *$

total samples $=227$

$\mathrm{x}$ range $=2.77132 \mathrm{E}+06 \quad 2.82341 \mathrm{E}+06$

$y$ range $=430386.498765$.

$\mathrm{z}$ range $=609.000 \quad 648.000$

initialize index array

transfer data to the index array

number entries into the index file $=227$

INCLUDE CROSS-SECTIONS

total samples $=380$

$\mathrm{x}$ range $=2.77132 \mathrm{E}+06 \quad 2.82318 \mathrm{E}+06$

$y$ range $=430386.498675$.

$\mathrm{z}$ range $=\quad 609.000 \quad 638.000$

initialize index array

transfer data to the index array 
number entries into the index file $=380$

CHANNEL_60ft

1

enter $x-y-z$ data input file name

bath_and_xsect.data

enter $\mathrm{z}$ bias value,(Add to Input $\mathrm{Z}$ Values)

0

Enter Minimum and Maximum $\mathrm{Z}$ value to be used in Interp 648,678

2

enter index grid spacing

600

enter $x$-dim , $y$-dim for index grid

105600,95100

icol $=176$ ilin $=159$

Enter Minimum X Coordinate

2770500

Enter Maximum Y coordinate

500500

3

Enter XY Input file of points to interpolate channel 60ftEN

Enter Output File Name

channel_60ftEN.xyzinterp

4

enter initial search radius (XY Input Units)

600

Enter MAX search radius factor

(multiply by initial search radius)

5

enter min sample count

5

enter pwr in weight function: $1 / \mathrm{r}^{* *}$ pwr

2

enter minumum $\mathrm{z}$ threshold value

0

5

enter max number points to read $(-1=$ all $)$ 
+ reading at line 500

+ reading at line 1000

total samples $=1255$

$\mathrm{x}$ range $=2.77206 \mathrm{E}+06 \quad 2.85373 \mathrm{E}+06$

$\mathrm{y}$ range $=413473.499627$.

$\mathrm{z}$ range $=648.000 \quad 678.000$

initialize index array

transfer data to the index array

+ transferring at line 500

+ transferring at line 1000

number entries into the index file $=1255$

channel_30ft

1

enter $x-y-z$ data input file name

bath_and_xsect.data

enter $\mathrm{z}$ bias value,(Add to Input $\mathrm{Z}$ Values)

0

Enter Minimum and Maximum $\mathrm{Z}$ value to be used in Interp 678,708

2

enter index grid spacing

600

enter $x$-dim , $y$-dim for index grid

105600,95100

icol $=176$ ilin $\doteq 159$

Enter Minimum X Coordinate

2770500

Enter Maximum Y coordinate

500500

3

Enter XY Input file of points to interpolate channel 30ftEN

Enter Output File Name

channel_30ftEN.xyzinterp

4

enter initial search radius (XY Input Units) 
600

Enter MAX search radius factor

(multiply by initial search radius)

5

enter min sample count

5

enter pwr in weight function: $1 / \mathrm{r}^{* *}$ pwr

2

enter minumum $\mathrm{z}$ threshold value

0

+ reading at line $\quad 500$

+ reading at line 1000

+ reading at line 1500

+ reading at line 2000

+ reading at line 2500

+ reading at line 3000

+ reading at line 3500

total samples $=3686$

$\mathrm{x}$ range $=2.77197 \mathrm{E}+06 \quad 2.87432 \mathrm{E}+06$

$\mathrm{y}$ range $=397974.499503$.

$\mathrm{z}$ range $=\quad 678.000 \quad 708.000$

initialize index array

transfer data to the index array

+ transferring at line 500

+ transferring at line 1000

+ transferring at line 1500

+ transferring at line 2000

+ transferring at line 2500

+ transferring at line 3000

+ transferring at line 3500

number entries into the index file $=3686$ 


\section{Appendix G}

Conversion of Centerline Countours to GRASS Sites File 



\section{Appendix G}

\section{Conversion of Centerline Countours to GRASS Sites File}

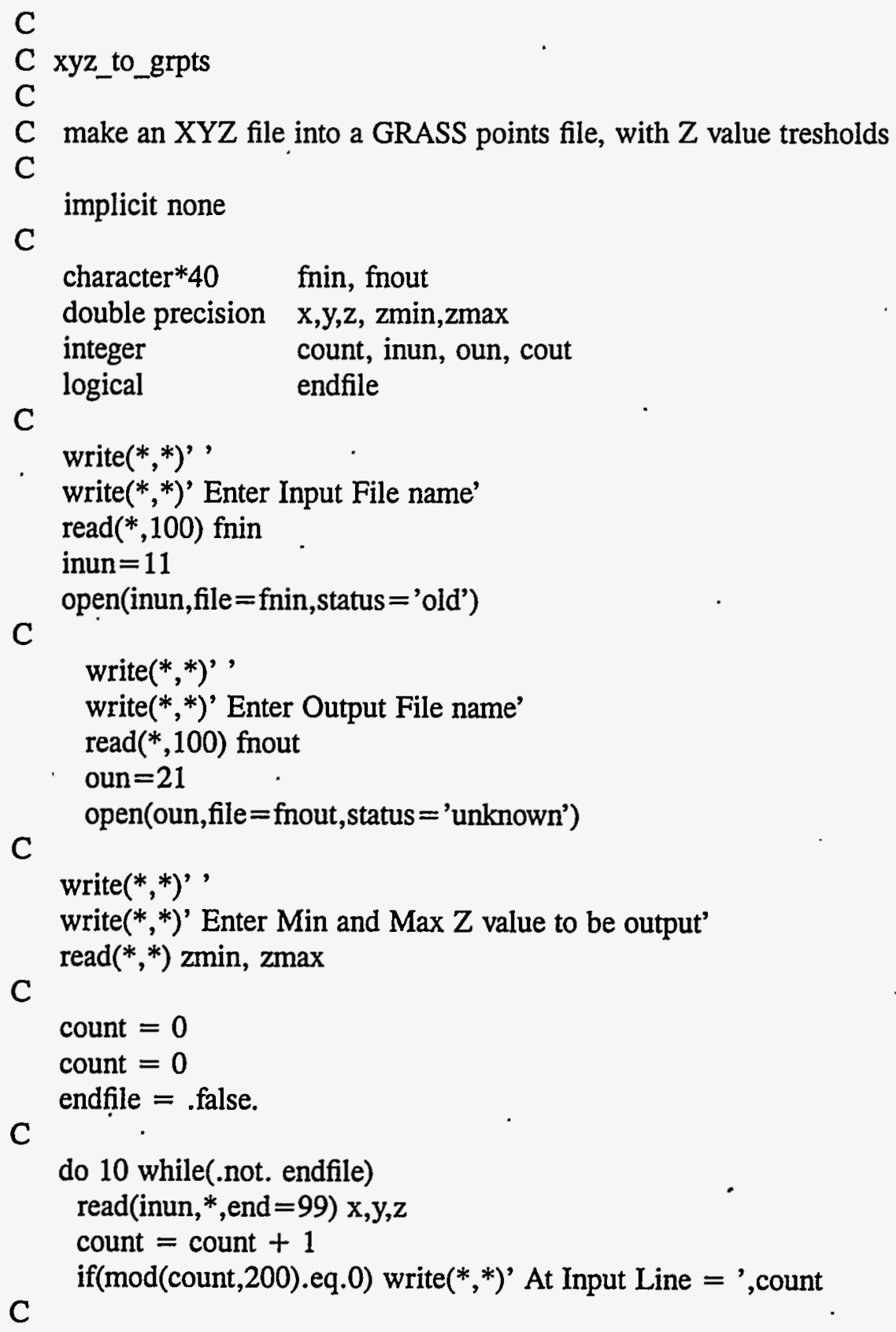


if(z.ge.zmin .and. z.le.zmax) then count $=$ count +1

write(oun,200) x,y,z

endif

C

if(endfile) then

99

endfile $=$.true

endif

10

continue

C

write $(*, *)^{\prime}$ Number lines read $=$ ', count

C

write $(*, *)^{\prime}$ Number of lines output $=$ ', cout

close(inun)

close(oun)

C

C $* * * * * * * * * * * * * * * * * * * * * * * * * * * * * *$

C Format Statements

$\mathrm{C}$

100

200

format(a40)

C

format $(2 \mathrm{x}, 2 \mathrm{f} 16.6,2 \mathrm{x}, \mathrm{f12} .5)$

stop

end 


\section{Appendix $\mathbf{H}$}

Replacement of 1989 Sounding Data Using 1992 Hydroacoustic Data for Schultz Bar and the Dredge Disposal Site, Creation of xyz Coordinates from Soundings 



\section{Appendix $\mathbf{H}$}

\section{Replacement of 1989 Sounding Data Using 1992. Hydroacoustic Data for Schultz Bar and the Dredge Disposal Site, Creation of xyz Coordinates from Soundings}

/* This program will read two different formats of dredge line depth soundings files.

* The program pulls the data from the input files only if the data exists in the

* current section of the river defined by station numbers. The program requires the

* input of two points with associated Eastings, Northings, and station numbers, for

* defining the control line of the river. The output of this program is the xyz

* coordinates of all points in the defined sections, and a grass-readable vector

* file which must have a header added to the beginning of the file first through.

* Currently, the two formats readable are GR format and fix format. *I

/* Date: 4-30-93 Output format added: Arc-info xyz format: one xy file and one $\mathrm{z}$ file. *I

\#include <stdio.h $>$

\#include <math.h $>$

FILE *infile, *outfile, *outfile2;

int center_mask $=0$;

int cen_base1, cen_base2;

float cen_offset1, cen_offset2;

double nor1, nor2, eas 1 , eas2;

get_center_data()

\{

char cenbuf[256];

printf ("InEnter center line data.");

printf ("InEnter station number for point 1: ");

gets (cenbuf, "\%s");

sscanf (cenbuf, "\%d $+\% \mathrm{f}^{\prime}$, \&cen_base1, \&cen_offset1);

printf ("InEnter Northing for station \%d+\%f: ", cen_base1, cen_offset1);

gets (cenbuf, "\%s");

sscanf (cenbuf, "\%lf", \&nor1);

printf ("InEnter Easting for station \%d+\%f: ", cen_base1, cen_offset1);

gets (cenbuf, "\%s");

sscanf (cenbuf, "\%lf", \&eas1);

printf ("InEnter station number for point 2: "); 


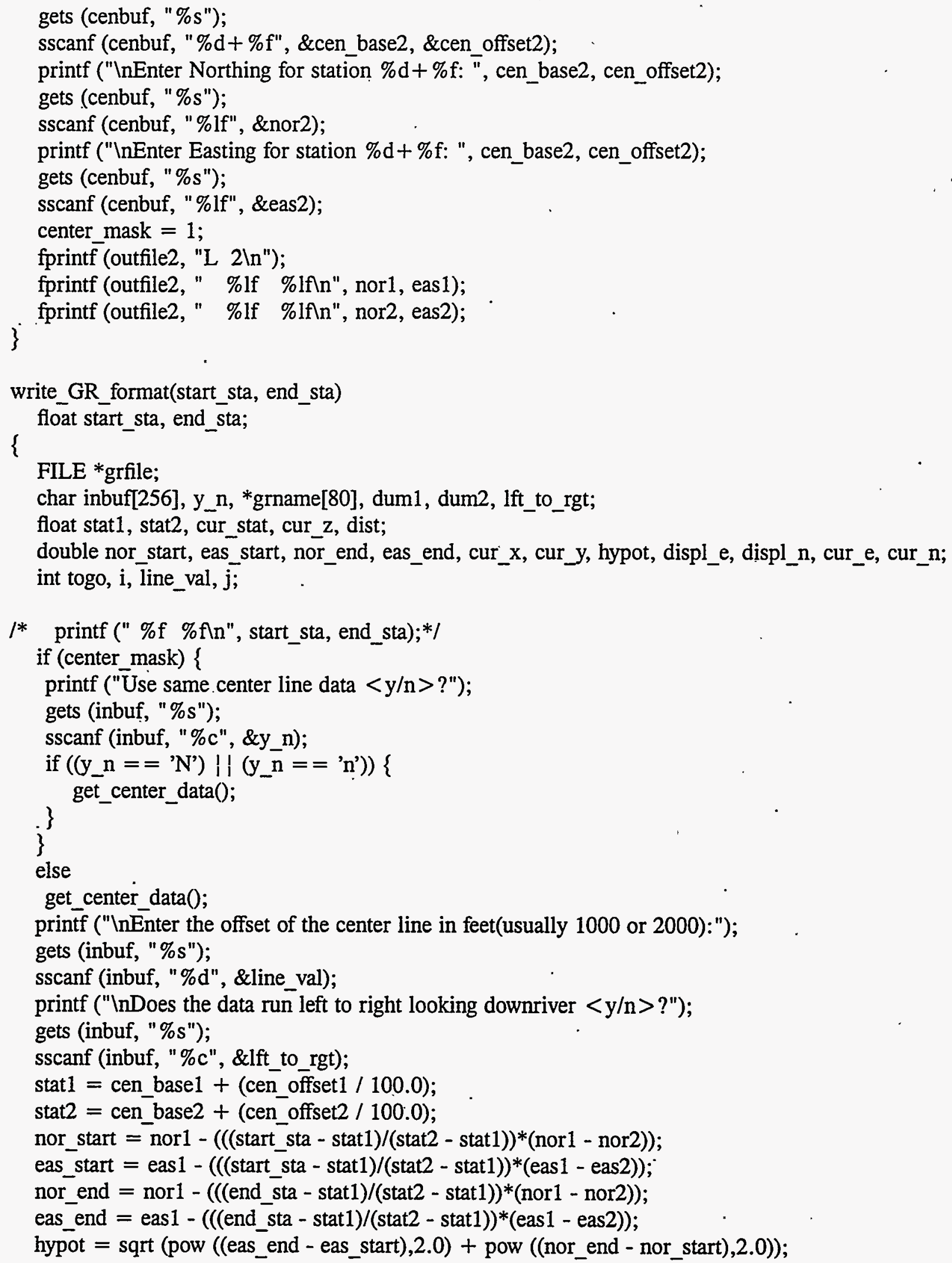


/* printf ("In\%lf \%lf \%lf \%lf \%lfln", nor_start, eas_start, nor_end, eas_end, hypot);*/ printf ("InEnter the GR format input filename: ");

gets (inbuf, "\%s");

sscanf (inbuf, "\%s", grname);

while ((grfile $=$ fopen(grname, "r")) $==$ NULL) \{

printf ("Unable to open \%sln.", grname);

printf ("InEnter the GR format input filename: ");

gets (inbuf, "\%s");

sscanf (inbuf, "\%s", grname);

\}

while (fgets (inbuf, 256, grfile)) \{

$1^{*}$ printf ("Inbuf: \%s|ln", inbuf);*/

sscanf (inbuf, "\%c\%c \%f \%d", \&dum1, \&dum2, \&cur_stat, \&togo);

while (dum1 $==$ 'C') \{

fgets (inbuf, 256, grfile);

\}

. sscanf (inbuf, "\%c\%c \%f \%d", \&dum1, \&dum2, \&cur_stat, \&togo);

$l^{*}$ printf ("Header: \%f \%dln", cur_stat, togo);*I

if ((cur_stat $>=$ start_sta) \&\& (cur_stat $<=$ end_sta)) \{

cur_y $=$ nor_start - $((($ cur_stat - start_sta $) /($ end_sta - start_sta $)) *($ nor_start - nor_end $))$;

cur_x = eas_start - $((($ cur_stat - start_sta $) /($ end_sta - start_sta $)) *($ eas_start - eas_end $))$;

fprintf (outfile2, "L \% din", togo);

$\mathrm{i}=0$;

while $(\mathrm{i}<$ togo) \{

fgets (inbuf, 20, grfile);

sscanf (inbuf, "\%c\%c \%f \%f", \&dum1, \&dum2, \&cur_z, \&dist);

/*

printf ("\%f \%fln", cur_z, dist);*I

displ_e = (line_val - dist)*(nor_end - nor_start)/hypot;

displ_n $=($ line_val - dist)*(eas_end - eas_start)/hypot;

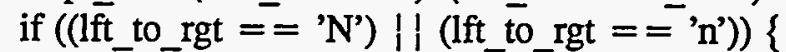

cur_e $=$ cur_x + displ_e;

\}

cur_n = cur_y - displ_n;

else \{

cur_e $=$ cur_x - displ_e;

\}

cur_n $=$ cur_y + displ_n;

fprintf (outfile, "\%18.8lf \%18.81f \%6.1fin", cur_e, cur_n, cur_z); fprintf (outfile2, " \%18.8If \%18.81f $\bar{n} "$, cur_n, cur_e);

$\mathrm{i}++$;

$\mathrm{j}=0$;

while $((j<4) \& \&(i<$ togo $))\{$

fgets (inbuf, 17, grfile);

sscanf (inbuf, " \%f \%f", \&cur_z, \&dist);

$I^{*} \quad$ printf ("\%f \% fln", cur_z, dist);*I

displ_e $=($ line_val - dist)*(nor_end - nor_start)/hypot;

displ_n $=($ line_val - dist $) *($ eas_end - eas_start $) /$ hypot; 


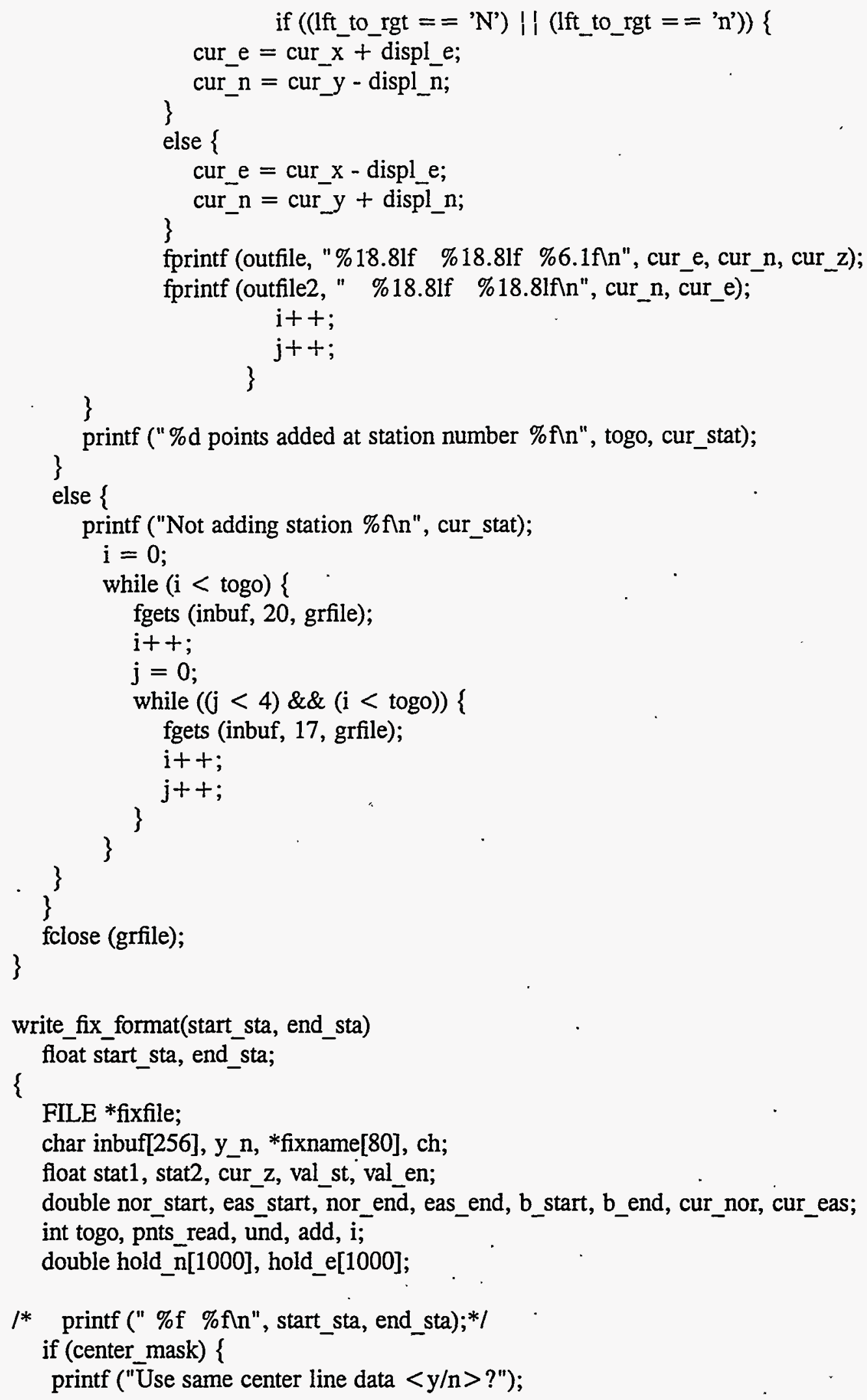




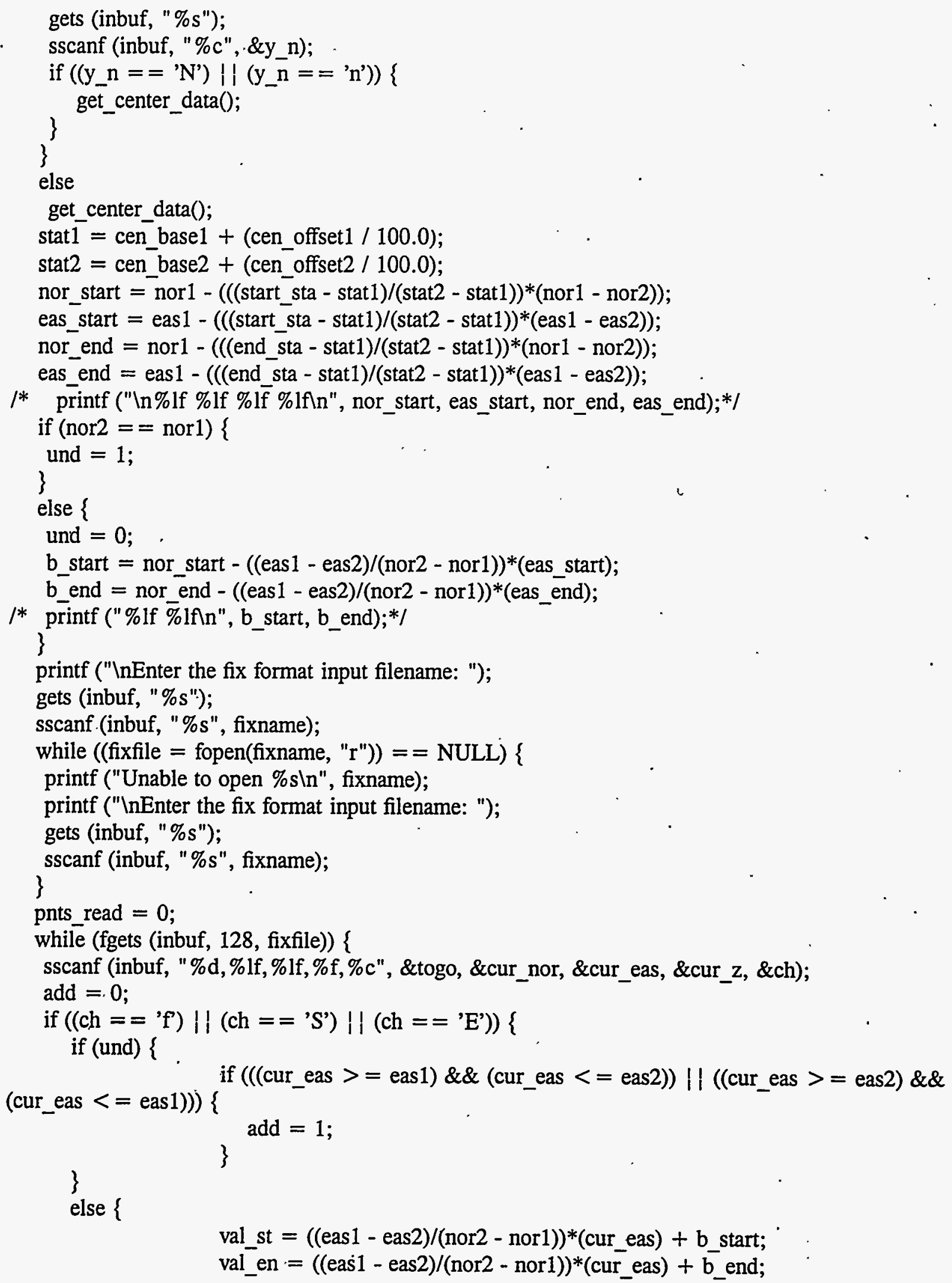




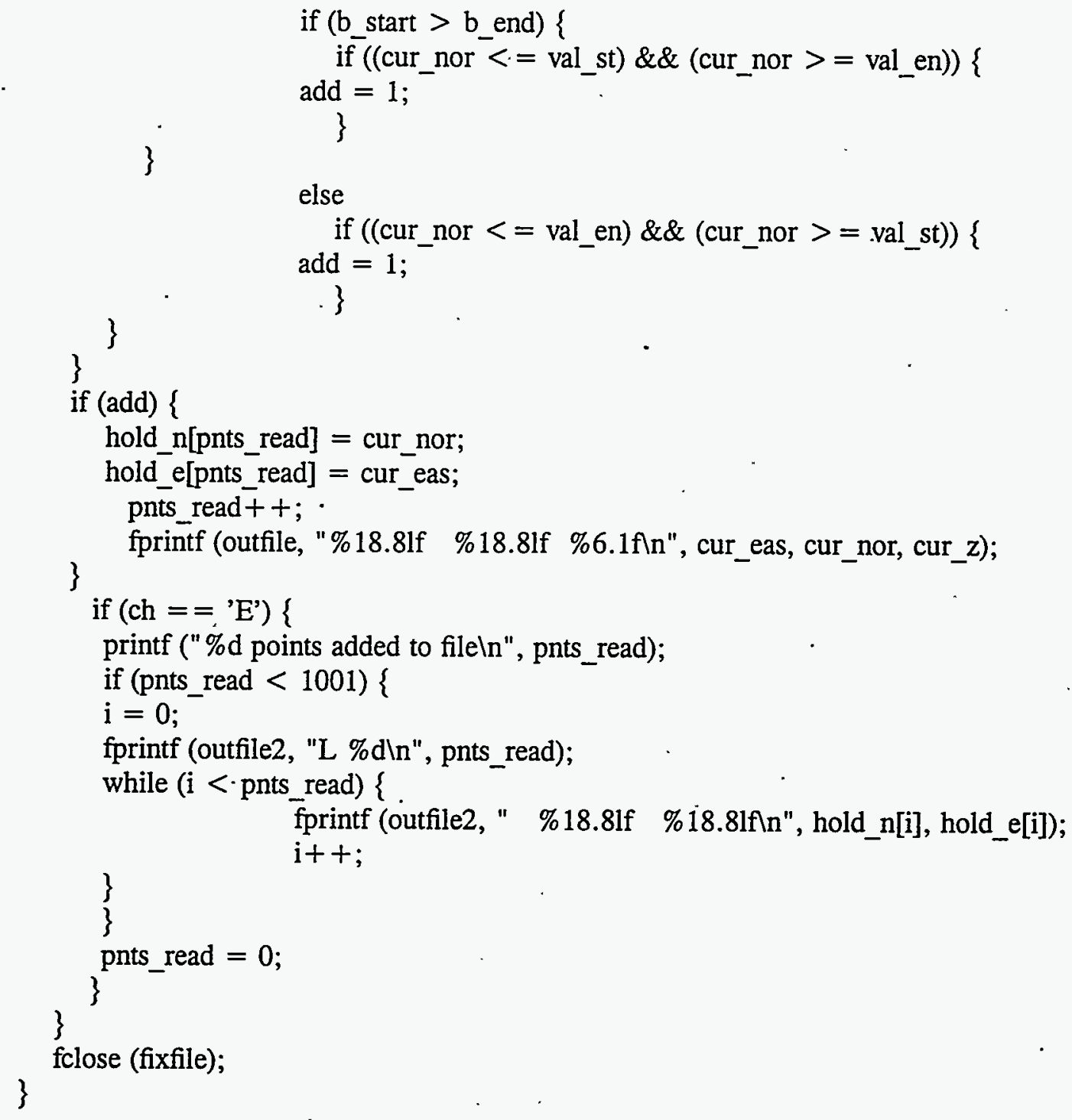


int base1, base2, choice;

float offset1, offset2;

printf ("Enter the output filename: ");

gets (buf, "\%s");

sscanf (buf, "\%s", outname);

while $\left(\left(\right.\right.$ outfile $=$ fopen(outname, $\left.\left." w^{\prime \prime}\right)\right)==$ NULL $)\{$

printf ("Unable to open \%s\n", outname);

printf ("Enter the output filename: ");

gets (buf, "\%s");

\}

sscanf (buf, "\%s", outname);

sprintf (outname2, "\%s.asc", outname);

outfile2 = fopen (outname2, "w");

printf ("InEnter the Base Line Station start number for section \# \%d:", cur_sect); gets (buf, "\%s");

sscanf (buf, "\%d+\%f", \&base1, \&offset1);

$l^{*} \quad$ printf ("\%d \%fn", base1, offset1);*I

while $\left(\left(\right.\right.$ cont $==$ 'y' $\left.^{\prime}\right) \|$ (cont $\left.\left.=={ }^{\prime} Y^{\prime}\right)\right)\{$

printf ("InEnter the Base Line Station end number for section \# \%d:", cur_sect);

gets (buf, "\%s");

sscanf (buf, "\%d+\%f", \&base2, \&offset2);

I* printf ("\%d \% fln", base2, offset2);*/

while ((base2 < base1) ||$($ (base2 $==$ base1) \&\& (offset1 $>=$ offset2))) \{

printf ("End point must be farther down river than start point! $n ")$;

printf ("Enter the Base Line Station end number for section \# \%d", cur_sect);

gets (buf, "\%s");

sscanf (buf, "\%d+\%f", \&base2, \&offset2);

$l^{*} \quad$ printf ("\%d \%fn", base2, offset2);*/

\}

printf ("InSelect format of input file for section \# \%dln", cur_sect);

printf ("In 1) GR format: X1 $0.00 \quad 118 \backslash \mathrm{n} "$ );

printf (" $\quad$ GR 667.5 $\quad 1014.666 .4$ 1021. $\quad 664.2$ 1031. 662.4 1041. 660.9

1051. In");

printf ("In");

printf (" 2) fix format: $1,464350.200,2811270.000,600.000, \operatorname{REF} \quad 20.00 \quad 1000.00 \mathrm{ln}$ ");

printf ("

$591,466242.34130022,2809494.57349555,664.8$, STARL 20.00 fix 518

11:44:04ln");

printf ("

printf ("\n");

590,466235.84130022,2809498.57349555,664.8,fix 517 11:44:03ln");

printf ("Number:");

gets (buf, "\%s");

sscanf (buf, "\%d", \&choice);

while $(($ choice $<1)$ || (choice > 2)) \{

printf ("InNot a valid choice. In");

printf ("Select format of input file for section \# \%dln", cur_sect);

printf ("In 1) GR format: X1 $0.00 \quad 118 \backslash \mathrm{n} ")$; 


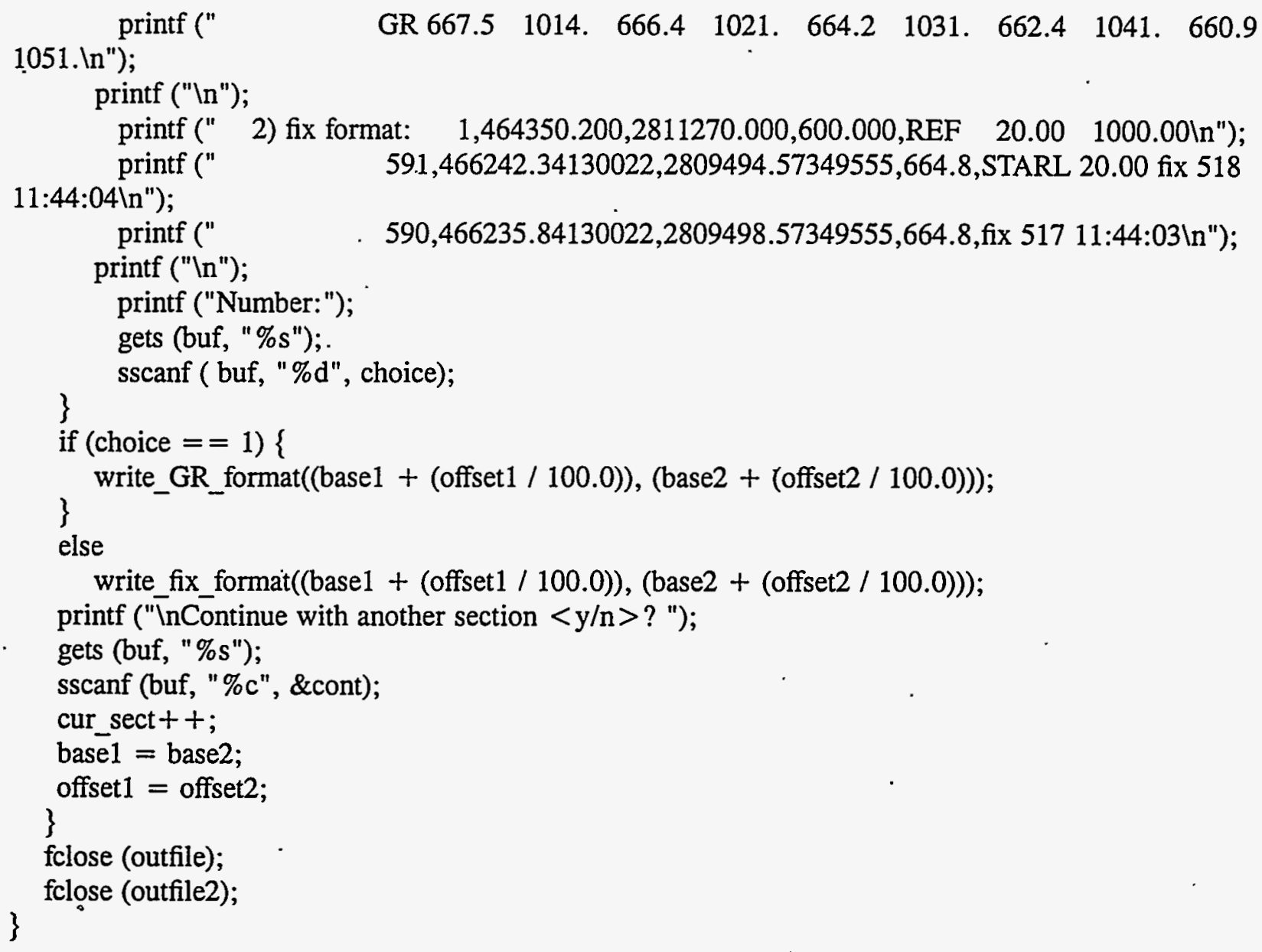




\section{Appendix I}

Replacement of 1989 Sounding Data Using 1992

Hydroacoustic Data for Schultz Bar and the Dredge Disposal Site, Creation of

Higher Resolution File 



\section{Appendix I}

\section{Replacement of 1989 Sounding Data Using 1992 Hydroacoustic Data for Schultz Bar and the Dredge Disposal Site, Creation of Higher Resolution File}

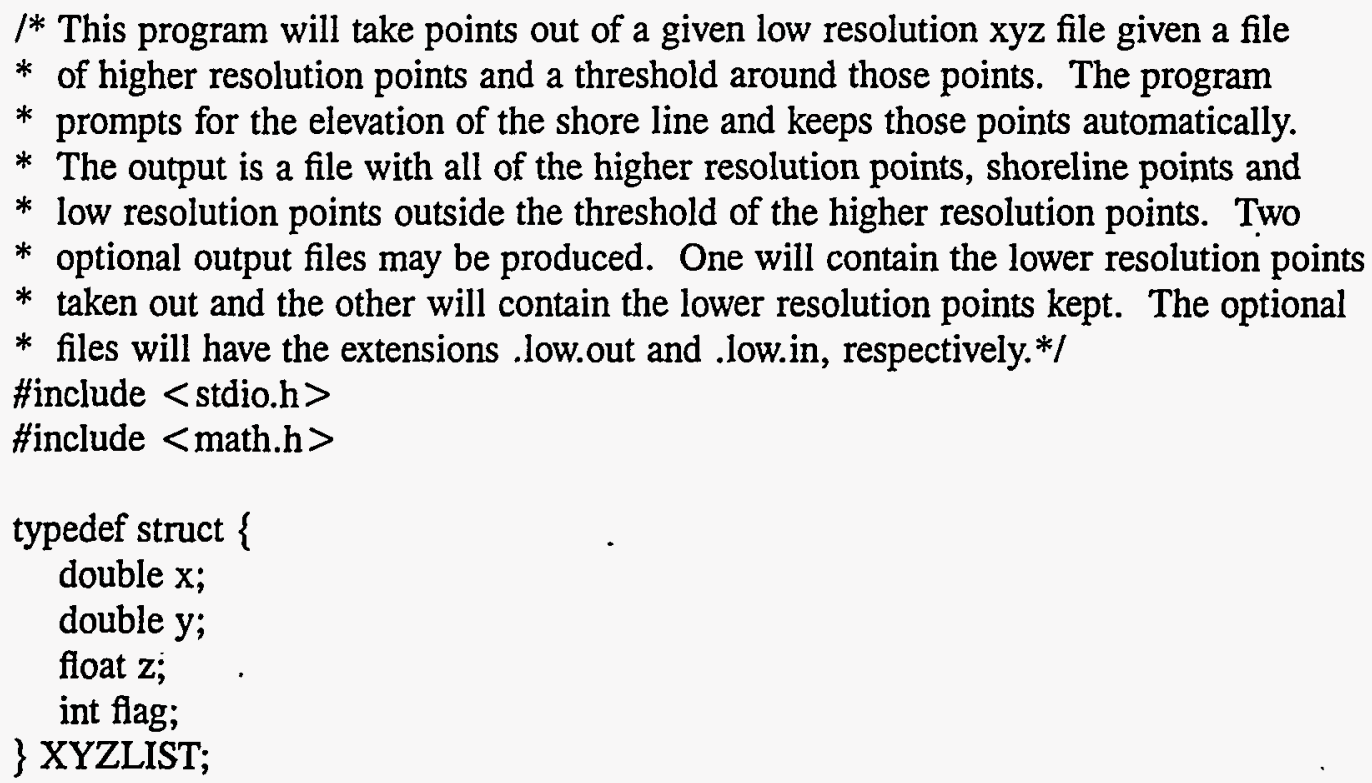

FILE *infile, *outfile, *outfile2;

int num, trans;

XYZLIST old_vals[100000];

int in_out $=\overline{0}$;

load_low_res (pool_ele) \{ fioat pool_ele;

char inbuf[128];

double $\mathrm{x}$ _val, y_val;

float z_val;

num $=0$;

trans $=0$;

while (fgets (inbuf, 128, infile)) \{

sscanf (inbuf, "\%lf \%lf \%f", \&x_val, \&y_val, \&z val); 


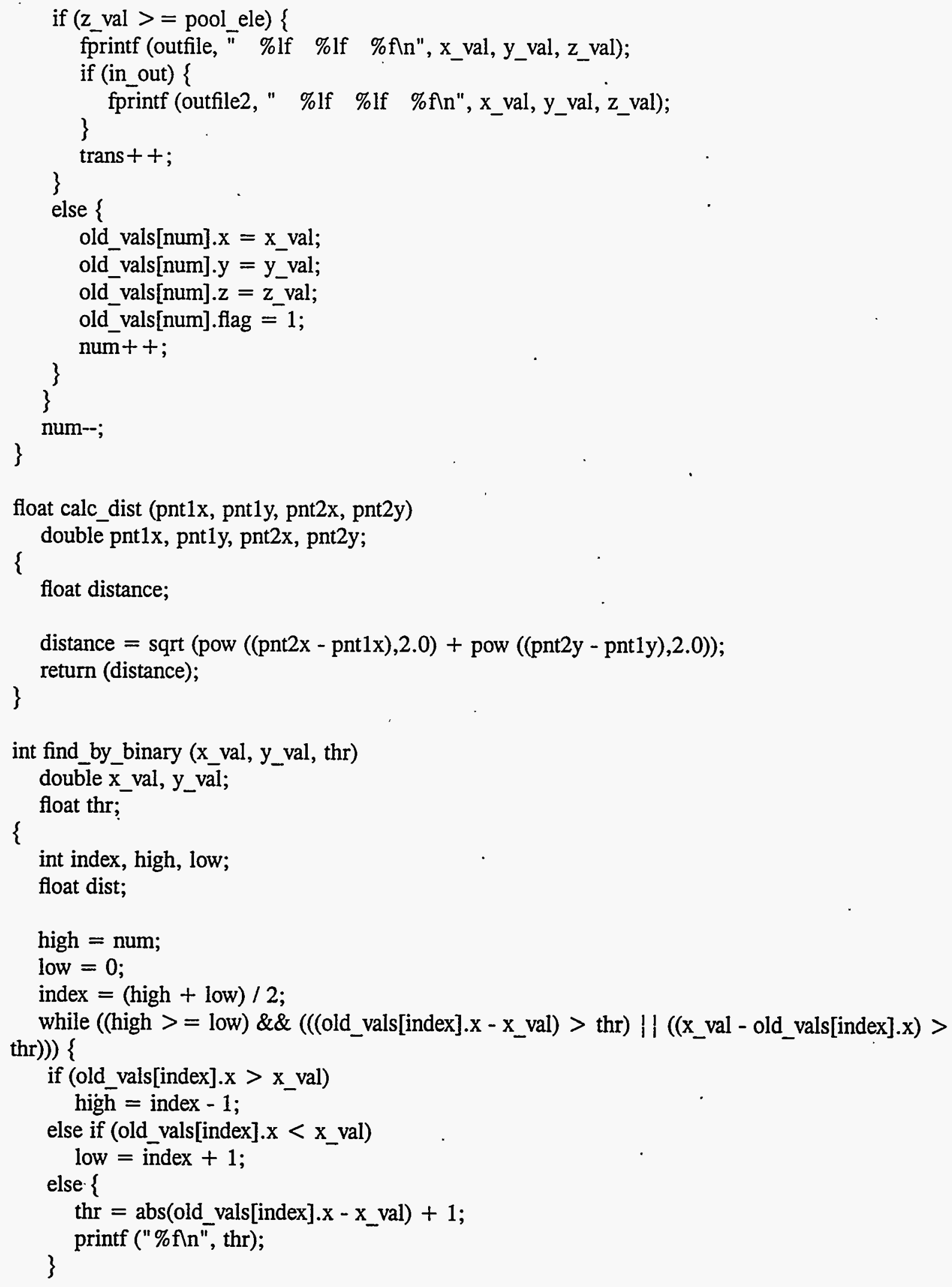




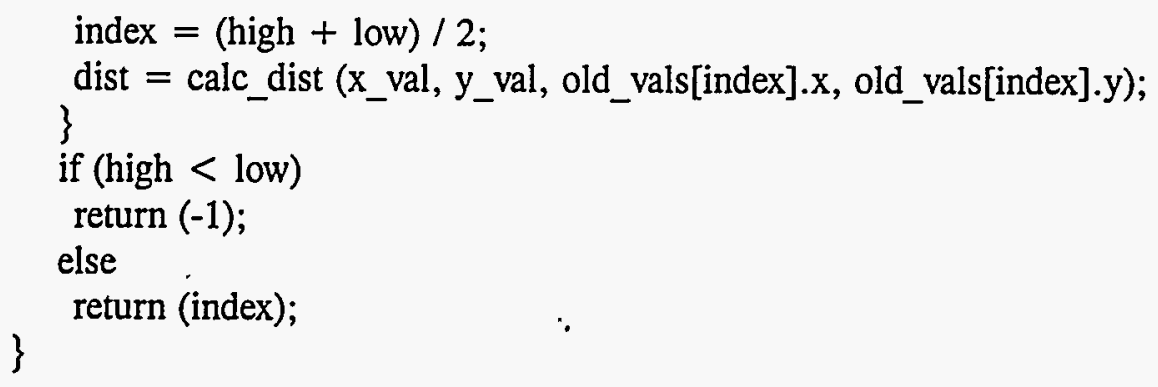


double $x_{-}$hi, y_hi;

float thres, z hi, pool;

printf ("InEnter the output filename: ");

gets (buf, "\%s");

sscanf (buf, "\%s", outname);

while $(($ outfile $=$ fopen(outname, "w")) $==$ NULL $)\{$

printf ("InUnable to open \%sln", outname);

printf ("Enter the output filename: ");

gets (buf, "\%s");

\}

sscanf (buf, "\%s", outname);

printf ("InGenerate low resolution files with kept and thrown away points $<y / n>$ ?");

gets (buf, "\%s");

sscanf (buf, "\%c", \&ch);

if $\left(\left(\mathrm{ch}=={ }^{\prime} \mathrm{Y}^{\prime}\right) \|\left(\mathrm{ch}=={ }^{\prime} \mathrm{y}^{\prime}\right)\right)\{$

in_out $=1$;

sprintf (out2name, "\%s.low.in", outname);

outfile2 = fopen (out2name, "w");

\}

printf ("InEnter the low resolution data filename: ");

gets (buf, "\%s");

sscanf (buf, "\%s", inname);

sprintf (syscmd, "sort \%s > foo1", inname);

while ((system (syscmd)) $==127)\{$

printf ("InUnable to find \% $\%$ n", inname);

printf ("Enter the low resolution data filename: ");

gets (buf, "\%s");

sscanf (buf, "\%s", inname);

sprintf (syscmd, "sort \%s > fool", inname);

\}

sprintf (inname, "fool");

infile = fopen (inname, " $r ")$;

printf ("InEnter the pool elevation in feet: ");

gets (buf, "\%s");

sscanf (buf, " \%f", \&pool);

printf ("InLoading low resolution file...");

load_low_res (pool);

printf ("In\%d points read in. In", num + trans);

fclose (infile);

printf ("InEnter the high resolution data filename: ");

gets (buf, "\%s");

sscanf (buf, "\%s", inname);

while $($ infile $=$ fopen (inname, "r")) $==$ NULL) \{

printf ("InUnable to find \%sln", inname);

printf ("Enter the high resolution data filename: "); 


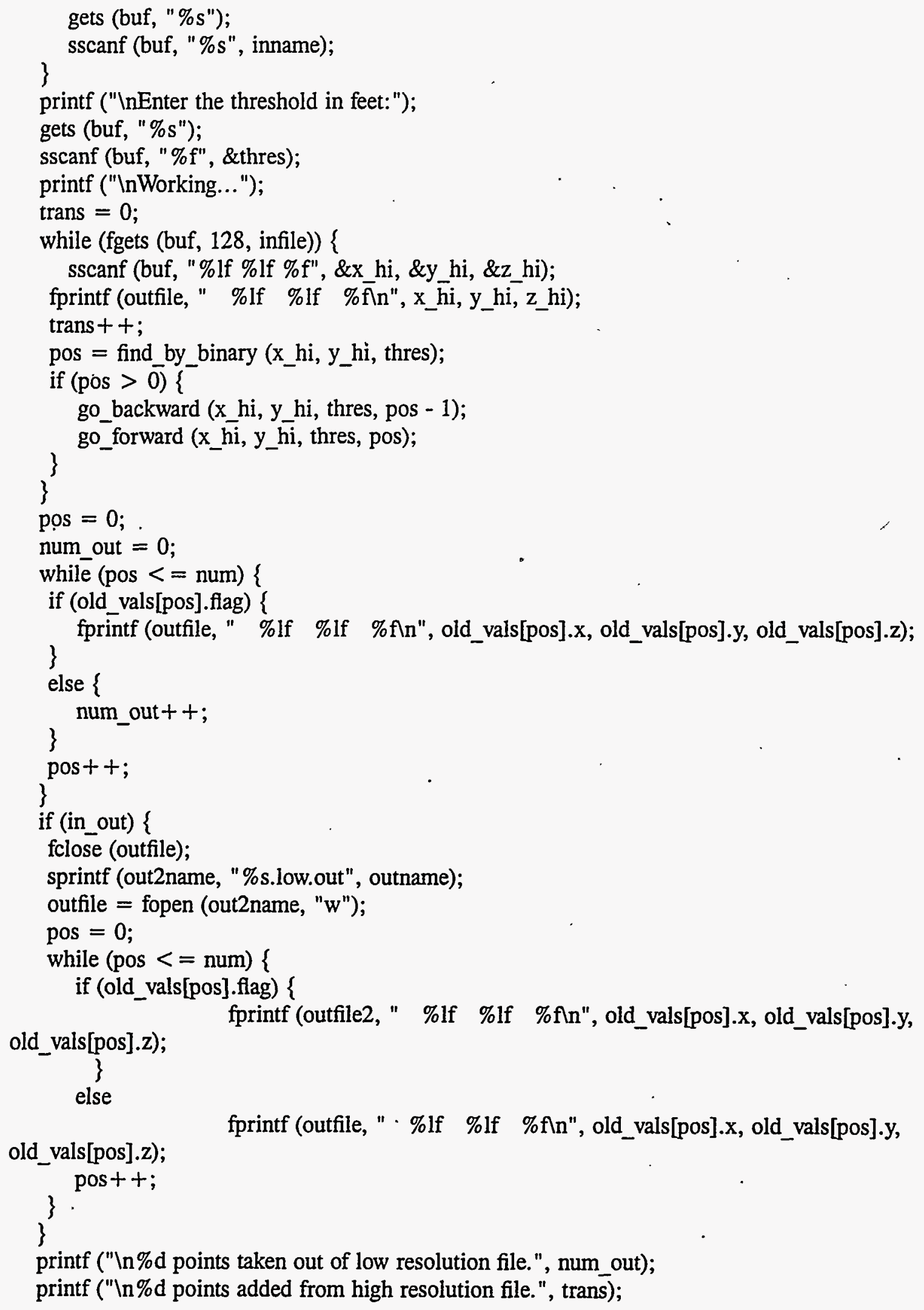


printf ("InDoneln");

system ("rm fool");

fclose (outfile);

\}

fclose (outfile2); 


\section{Appendix J}

Codes for Fish Species 



\section{Appendix $\mathbf{J}$}

\section{Codes for Fish Species}

List of common names, scientific names, and species codes for fishes in Lower Granite Reservoir, Idaho-Washington (Bennett et al. 1990).

\section{CODES}

LTR

ASA

ATR

ONE

OTS

PWI

OMY

AAL

CCA

MCA

POR

ROS

RBA

CCO

CMA

INA

INE

IME

IPU

LGI

LMA

LSP

PNI

PAN

PSP

MDO

PFL

COT
SCIENTIFIC NAME

Lampetra trientatus

Alosa sapidissima

Acipenser transmontanus

Oncorhyncus nerka

Oncorhyncus tshawytscha

Prosoplum williamsoni

Oncorhyncus mykiss

Acrocheilus alutaceus

Cyprinus carpio

Mylocheilus caurinus

Ptychoshellus oregonenisis

Rhinichthys osculus

Richardsonius balteatus

Catostomus columbianus

Catostomus macrocheilus

Ictalurus natalis

Ictalurus nebulosus

Ictalurus melas

Ictalurus punctatus

Lepomis gibbosus

Lepomis macrochirus

Lepomis spp.

Pomaxis nigromaclatus

Pomaxis annularis

Pomaxis spp.

Micropterus dolomieui

Perca flavescens

Cottus spp.

\section{COMMON NAME}

pacific lamprey

american shad

white sturgeon

sockeye salmon

chinook salmon

mountain whitefish

rainbow trout

chiselmouth

carp

peamouth

northern squawfish

specticled dace

redside shiner

bridgelip sucker

largescale sucker

yellow bullhead

brown bullhead

black bullhead

channel catfish

pumpkinseed

bluegill

misc. juv. sunfish

black crappie

white crappie

misc. juv. crappie

smallmouth bass

yellow perch

sculpin 



\section{Appendix K}

\section{HEC-6 Sediment Properties}





\section{Appendix K}

\section{HEC-6 Sediment Properties}

HEC-6 description of sediment properties and transport functions.

\begin{tabular}{|c|c|c|c|}
\hline ID NUMBER & CLASSIFICATION & GRAIN SIZE (mm) & GEOMETRIC MEAN \\
\hline 1 & Very Fine Sand & $0.062-0.125$ & 0.088 \\
\hline 2 & Fine Sand & $0.125-0.250$ & 0.177 \\
\hline 3 & Medium Sand & $0.250-0.500$ & 0.354 \\
\hline 4 & Coarse Sand & $0.500-1.000$ & 0.707 \\
\hline 5 & Very Coarse Sand & $1.000-2.000$ & 1.414 \\
\hline 6 & Very Fine Gravel & $2.000-4.000$ & 2.828 \\
\hline 7 & Fine Gravel & $4.000-8.000$ & 5.657 \\
\hline 8 & Medium Gravel & $8.000-16.000$ & 11.314 \\
\hline 9 & Coarse Gravel & $16.000-32.000$ & 22.627 \\
\hline 10 & Very Coarse Gravel & $32.000-64.000$ & 45.255 \\
\hline
\end{tabular}





\section{Distribution}

No. of

Copies

OFFSITE

12 DOE/Office of Scientific and Technical Information

L. Cunningham

U.S. Corps of Engineers

Walla Walla District

Planning Division

Environmental Resource Branch 201 N. 3rd

Walla Walla, WA 99362-1876

T. Miller

U.S. Corps of Engineers

Walla Walla District

Planning Division

Environmental Resource Branch 201 N. 3rd

Walla Walla, WA 99362-1876

5 C. Pinney

U.S. Corps of Engineers

Walla Walla District

Planning Division

Environmental Resource Branch 201 N. 3rd

Walla Walla, WA 99362-1876
No. of

Copies

\section{ONSITE}

50 Pacific Northwest Laboratory

D. D. Dauble

K6-54

J. P. Duncan

K6-54

B. J. Evans (33)

K9-55

J. L. Gordon

$\cdot \mathrm{K} 2-55$

K. A. Hoover

K9-33

B. V. Mavros

K6-54

R. Mazaika

J. B. Moser

K9-55

D. A. Neitzel

K6-54

E. M. Perry

K9-55

K. L. Steinmaus

K9-55

Publishing Coordination

Technical Report Files (5)

\section{Routing}

R. W. Bryce

K9-34

R. M. Ecker

SE-UI

M. J. Graham

K9-38

S. A. Rawson

K9-34

R. L. Skaggs

K9-34

P. C. Hays (last) 
